\title{
Palladium-katalysierte allylische Alkylierung und Synthese von Pyrazolonen an der festen Phase
}

\author{
Dissertation \\ zur Erlangung des Doktorgrades \\ der Mathematisch-Naturwissenschaftlichen Fakultäten \\ der Georg-August-Universität zu Göttingen
}

vorgelegt von

Thomas Hippe

aus

Göttingen

Göttingen 1999 
D 7

Referent: Prof. Dr. Dr. h.c. L. F. Tietze

Korreferent: Prof. Dr. A. Zeeck

Tag der mündlichen Prüfung: 02.11.1999 
Die vorliegende Arbeit wurde in der Zeit von Januar 1997 bis September 1999 im Organisch-Chemischen Institut der Georg-August-Universität Göttingen unter Leitung von Prof. Dr. Dr. h.c. L. F. Tietze angefertigt.

Mein besonderer Dank gilt meinem Doktorvater, Herrn Prof. Dr. Dr. h.c. L. F. Tietze für die interessante Aufgabenstellung, die Möglichkeit zur Durchführung dieser Arbeit und die vielen Anregungen in zahlreichen Diskussionen. 
Für Nadège 


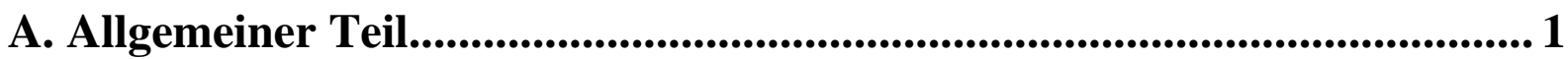

1. Einleitung ........................................................................................................................................ 1

2. Synthesen an der festen Phase ......................................................................................... 3

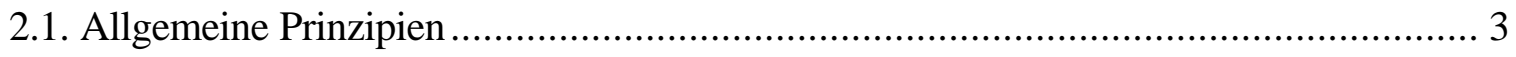

2.2. Harze und Linker......................................................................................... 5

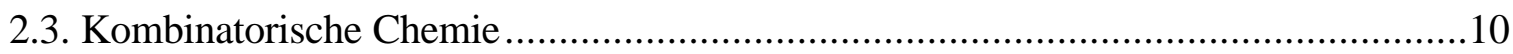

2.4. Beispiele kombinatorischer Festphasensynthese …............................................. 12

3. Palladium-katalysierte Reaktionen .................................................................................17

3.1. Palladium-katalysierte allylische Alkylierung ................................................... 17

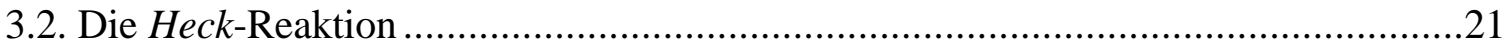

3.3. Ausgewählte Beispiele Palladium-katalysierter Reaktionen..................................24

4. Aufgabenstellung und Planung der Arbeit ..........................................................................28

5. Synthese der Edukte ..........................................................................................................30

5.1. Synthese von spacermodifiziertem Merrifield-Harz 6 ........................................ 30

5.2. Synthese der polymergebundenen 1,3-Dicarbonylverbindungen 138 und 143 ..........32

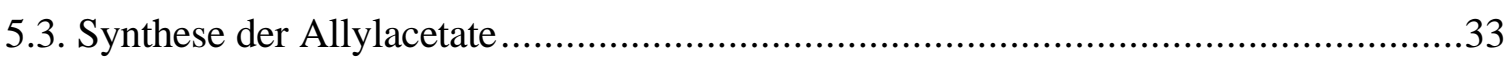

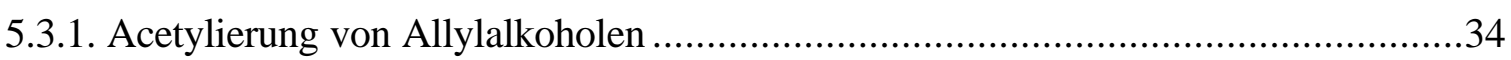

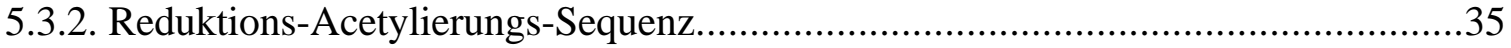

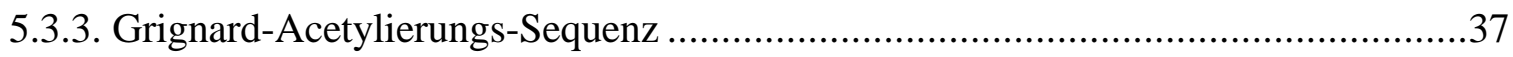

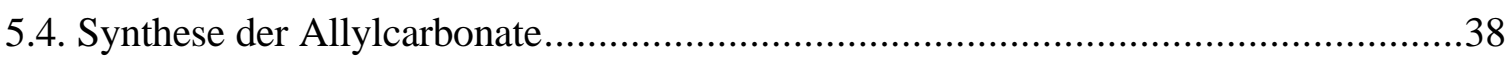

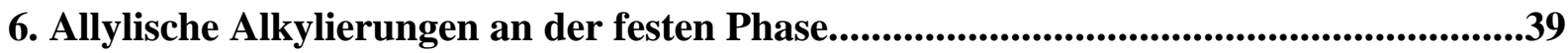

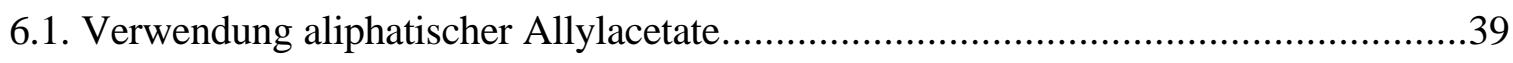

6.1.1. Diskussion ausgewählter spektroskopischer Daten .........................................43

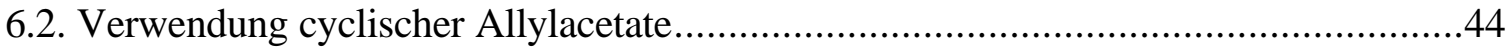

6.2.1. Diskussion ausgewählter spektroskopischer Daten ........................................46

6.3. Verwendung aromatischer und heteroaromatischer Allylacetate .............................47

6.3.1. Diskussion ausgewählter spektroskopischer Daten ..........................................51 


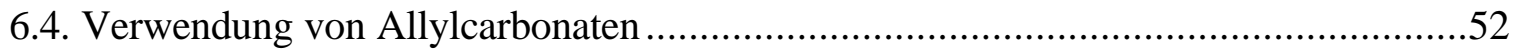

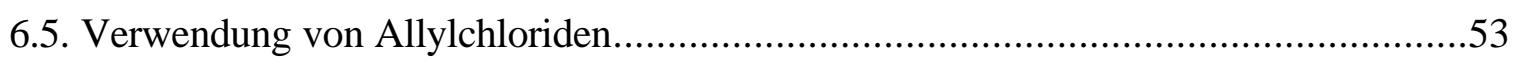

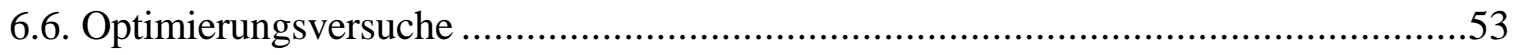

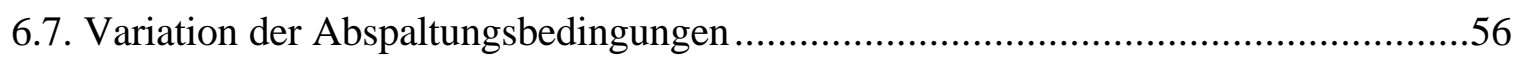

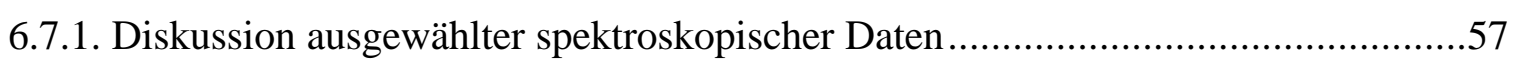

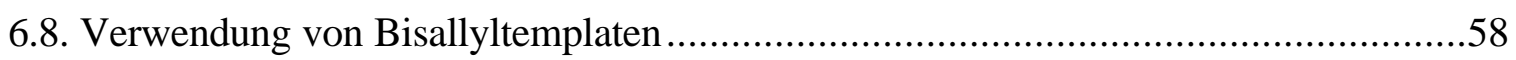

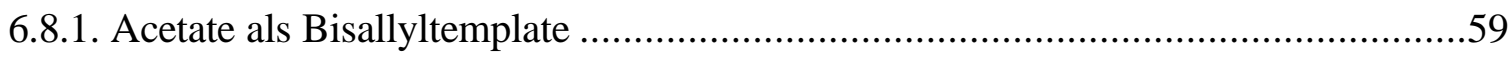

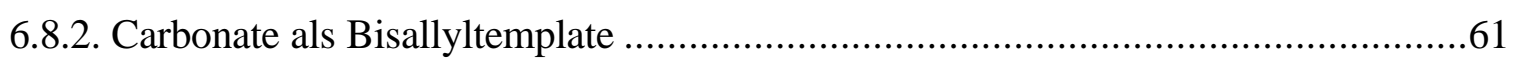

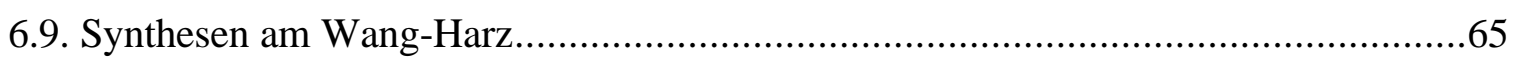

7. Heck-Reaktionen an der festen Phase ..........................................................................67

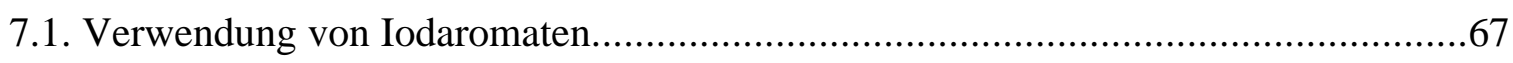

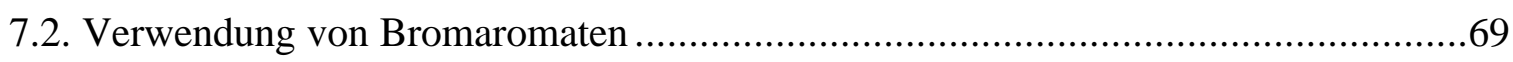

7.3. Diskussion ausgewählter spektroskopischer Daten............................................... 71

8. Festphasensynthese von Pyrazolonen....................................................................73

8.1. Diskussion ausgewählter spektroskopischer Daten........................................... 78

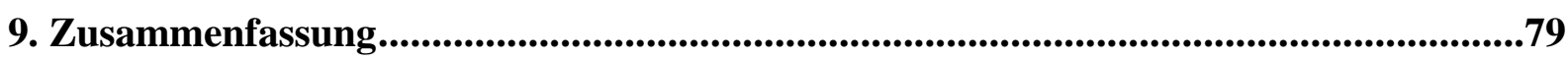

B. Experimenteller Teil ...................................................................................8

1. Allgemeine Methoden ....................................................................................................................88

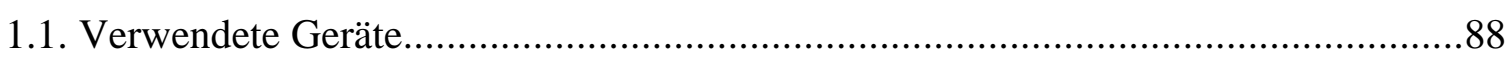

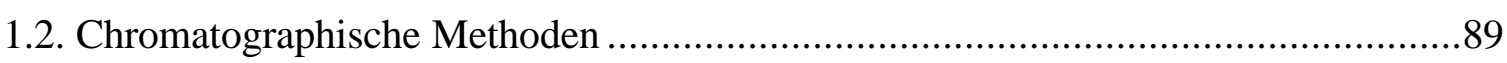

1.3. Allgemeine Bemerkungen zu Festphasenreaktionen .........................................89

2. Allgemeine Arbeitsvorschriften ...............................................................................91

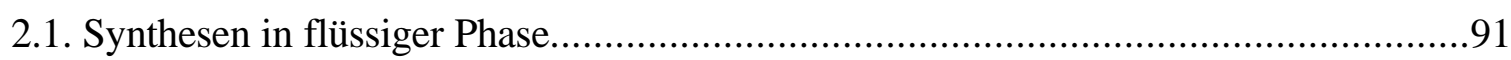

2.1.1. AAV 1: DIBAH-Reduktion von Enonen......................................................... 91

2.1.2. AAV 2: Grignard-Reaktion aromatischer Aldehyde.........................................91

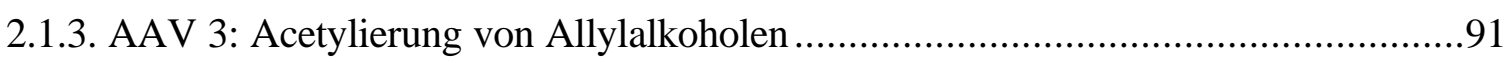

2.1.4. AAV 4: Darstellung von Carbonaten aus Allylalkoholen .....................................92

2.1.5. AAV 5: Allylische Alkylierung von 1,3-Dicarbonylverbindungen ........................92 
2.2. Synthesen am polymeren Träger

2.2.1. AAV 6: Allylische Alkylierung von 1,3-Dicarbonylverbindungen mit Allylacetaten

2.2.2. AAV 7: Allylische Alkylierung von 1,3-Dicarbonylverbindungen mit Allylcarbonaten

2.2.3. AAV 8: Reduktive Abspaltung polymergebundener 1,3-Dicarbonylverbindungen..93

2.2.4. AAV 9: Abspaltung von polymergebundenen 1,3-Dicarbonylverbindungen durch

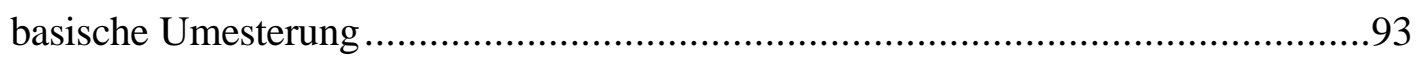

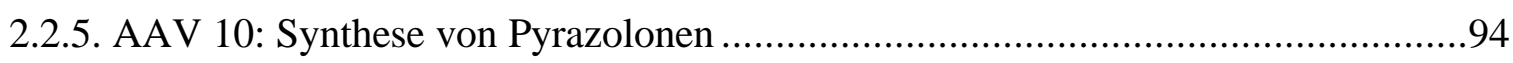

3. Synthesen in flüssiger Phase ..............................................................................................95

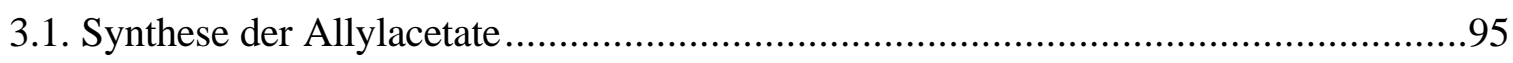

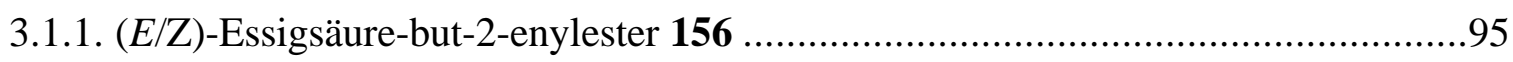

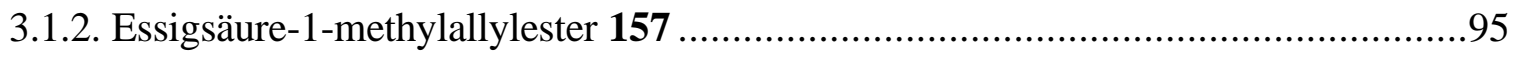

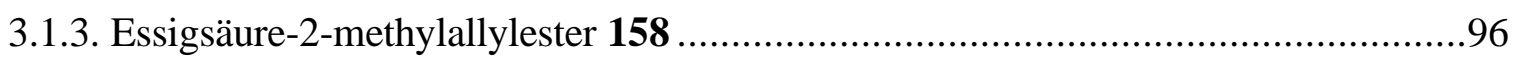

3.1.4. (E)-Essigsäure-5-(2,5-dimethoxy-3,4,6-trimethylphenyl)-3-methyl-pent -2-enyl-

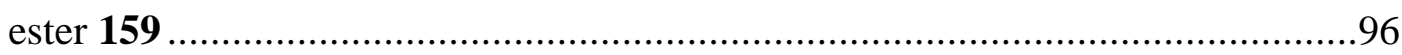

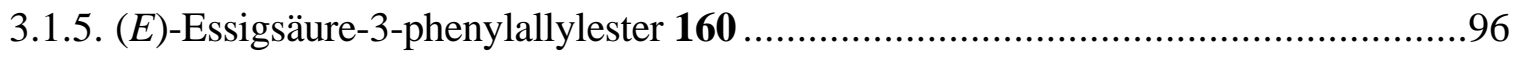

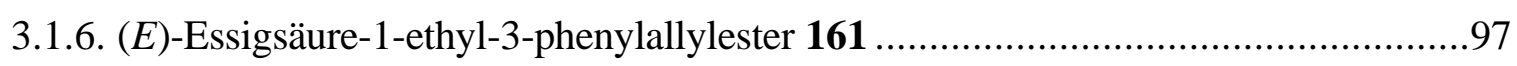

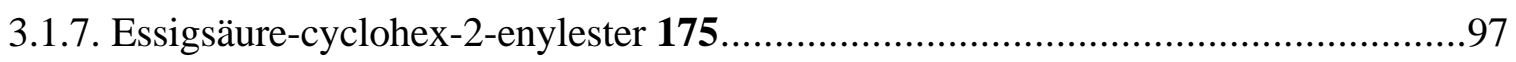

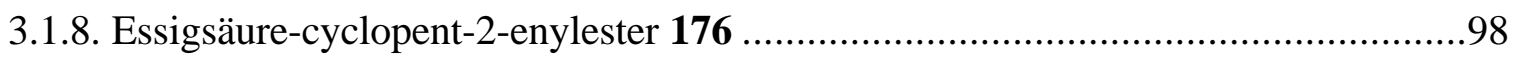

3.1.9. Essigsäure-2-methyl-cyclopent-2-enylester 177 .......................................... 98

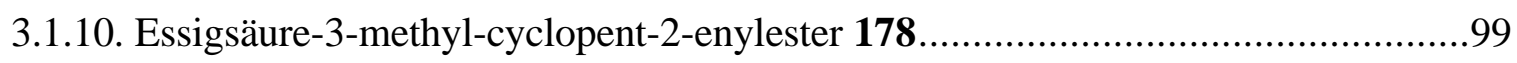

3.1.11. (E)-Essigsäure-3-(4-dimethylaminophenyl)-allylester 179 ..............................99

3.1.12. (E)-Essigsäure-3-(3-methoxyphenyl)-allylester 180 ....................................99

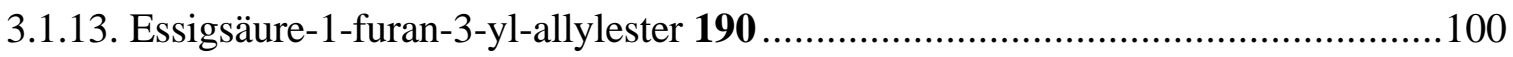

3.1.14. Essigsäure-1-thiophen-2-yl-allylester 191 ................................................. 101

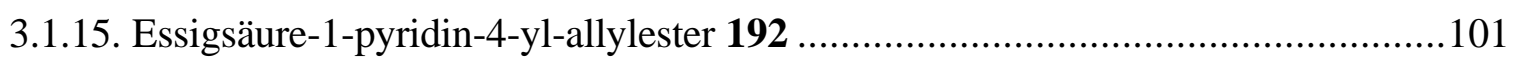

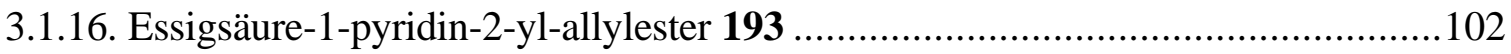

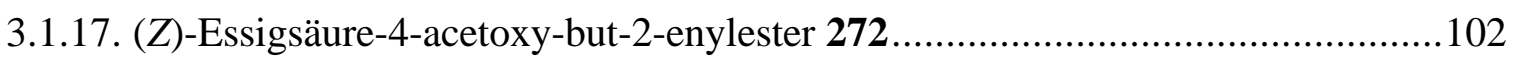

3.1.18. (Z)-Essigsäure-4-hydroxy-but-2-enylester 273 und (Z)-Essigsäure-4-acetoxy-

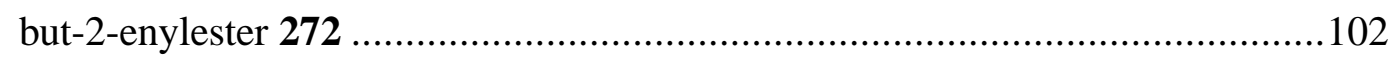

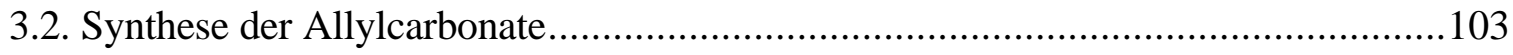

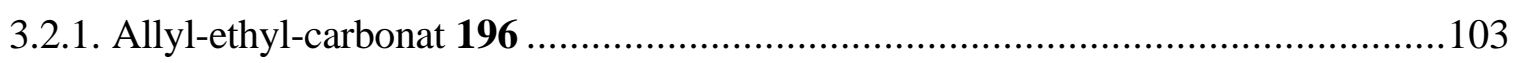

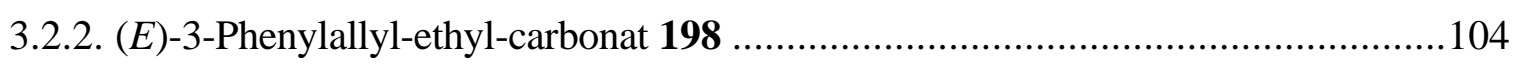


3.2.3. (Z)-Ethyl-4-hydroxybut-2-encarbonat 275 und (Z)-1,4-Bis(ethoxycarbonyloxy)-

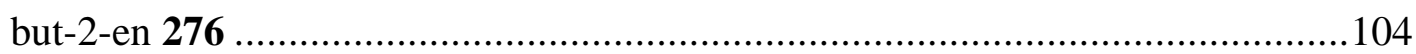

3.3. Palladium-katalysierte allylische Alkylierungen in flüssiger Phase .......................... 105

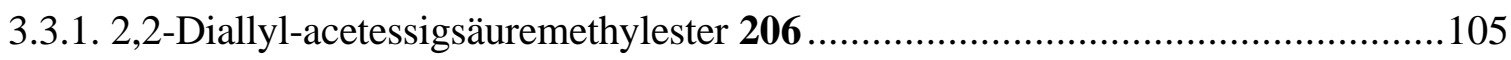

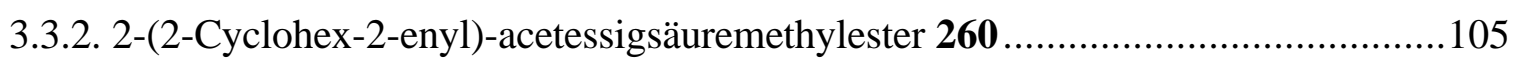

3.3.3. (E)-2-(4-Hydroxybut-2-enyl)-acetessigsäuremethylester 277 und (E)-2,2-Bis(4-

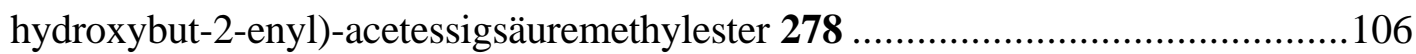

4. Synthesen an der festen Phase.....................................................................................107

4.1. Synthese von spacermodifiziertem Harz 6 .................................................... 107

4.2. Synthese des polymergebundenen 3,5-Dinitrobenzoesäureesters 131 .....................107

4.3. Synthese von polymergebundener Benzoesäure 133 ......................................... 107

4.4. Synthese von polymergebundenem Acetoacetat 138 .......................................... 108

4.5. Synthese von polymergebundenem Methylmalonat 143 ..................................... 108

4.6. Allylische Alkylierungen mit Allylacetaten an der festen Phase ............................. 108

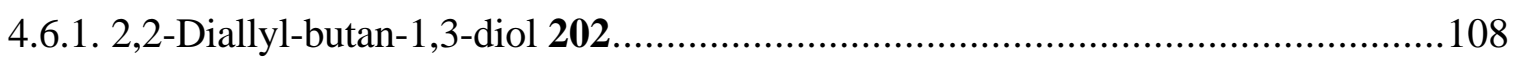

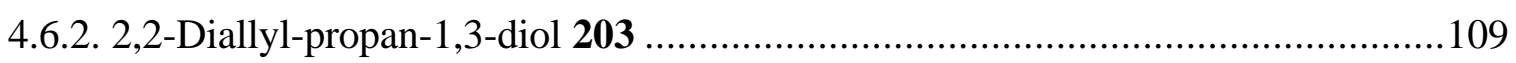

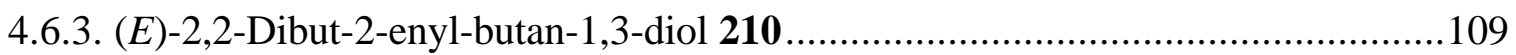

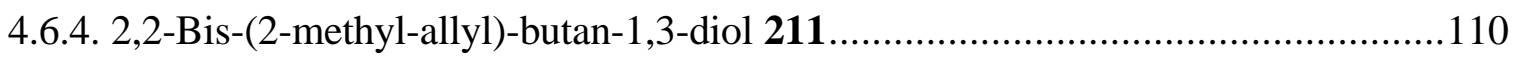

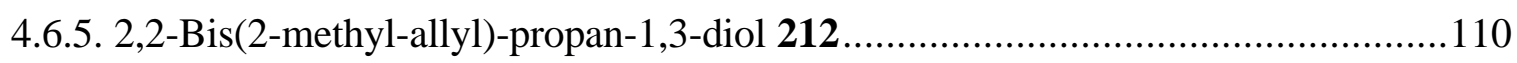

4.6.6. (E)-2-(3-Methyl-5-(2,5-dimethoxy-3,4,6-trimethylphenyl)-pent-2-enyl)-butan-

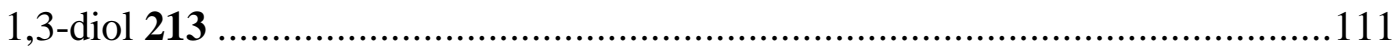

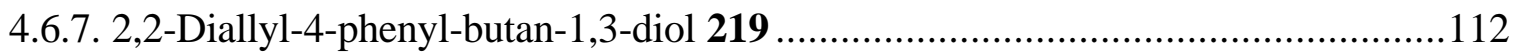

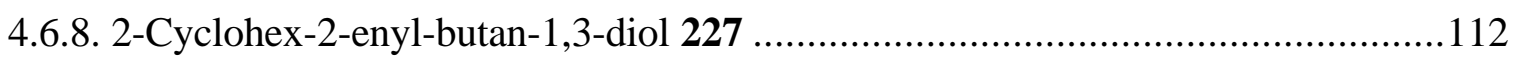

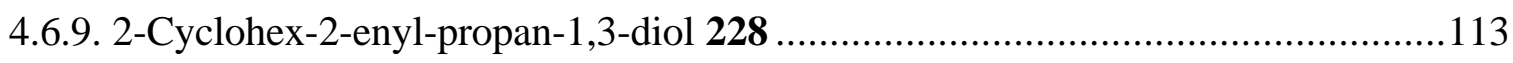

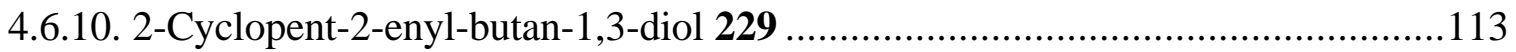

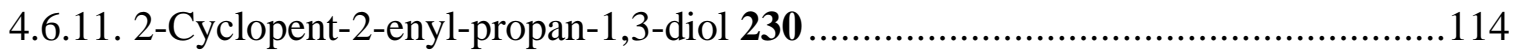

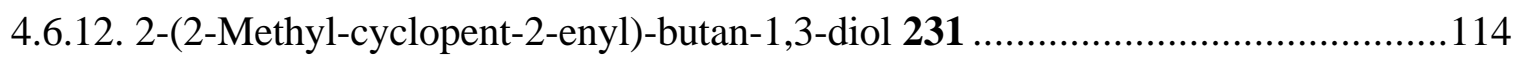

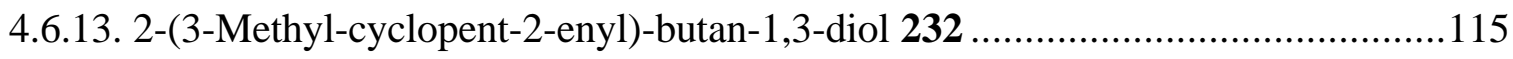

4.6.14. 2-(3-Methyl-cyclopent-2-enyl)-propan-1,3-diol 233 ....................................115

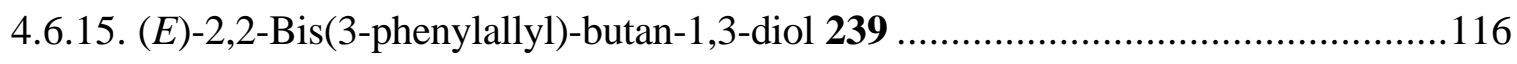

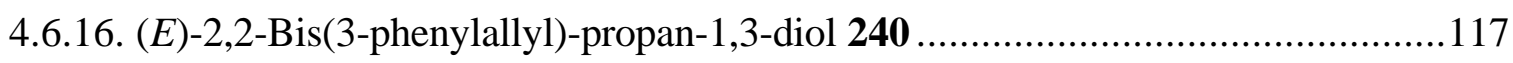

4.6.17. (E)-2,2-Bis(3-(4-dimethylaminophenyl)-allyl)-butan-1,3-diol 241 ......................117

4.6.18. (E)-2,2-Bis(3-(4-dimethylaminophenyl)-allyl)-propan-1,3-diol 242 .................. 118

4.6.19. (E)-2,2-Bis(3-(3-methoxyphenyl)-allyl)-butan-1,3-diol 243 ............................ 118

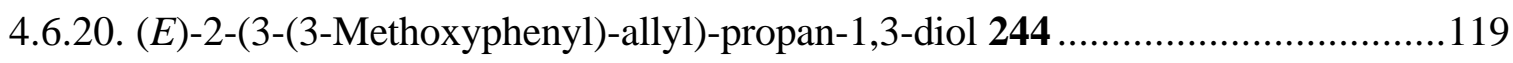




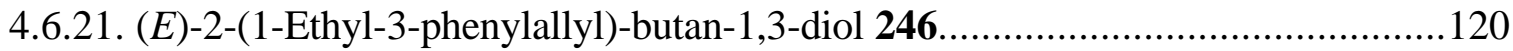

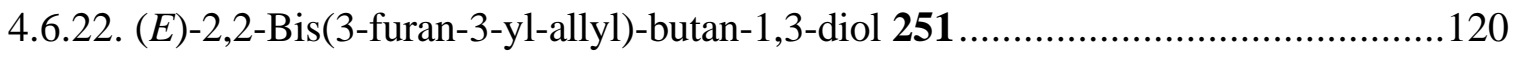

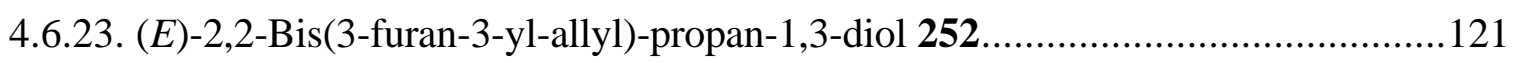

4.6.24. (E)-2,2-Bis(3-thiophen-2-yl-allyl)-butan-1,3-diol 253 .....................................121

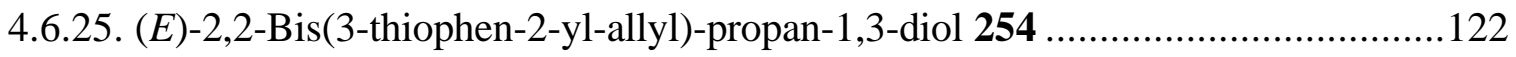

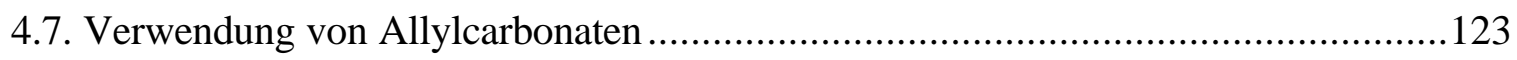

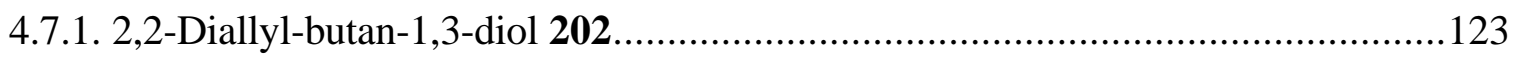

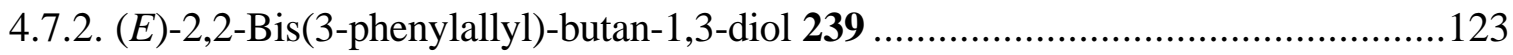

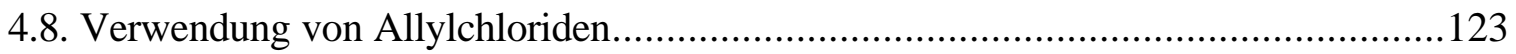

4.8.1. 2-(2-Methyl-allyl)-propan-1,3-diol 257 und 2,2-Bis(2-methyl-allyl)-propan-

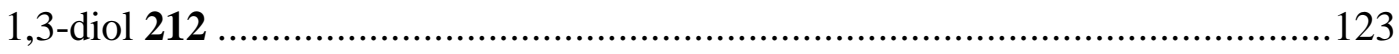

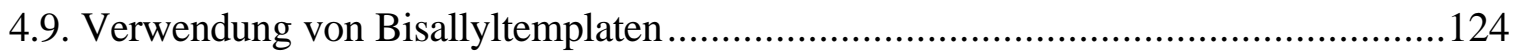

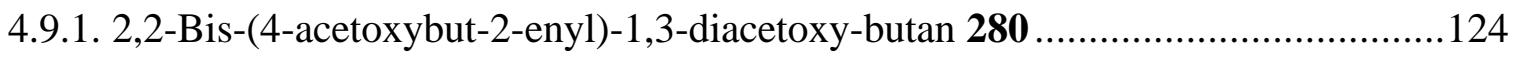

4.9.2. Synthese der polymergebundenen (E)-2,2-Bis(4-Acetoxy-but-2-enyl)-aceto-

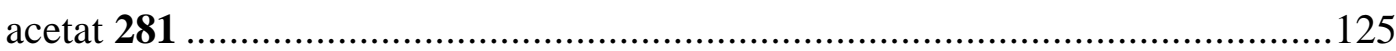

4.9.3. (E)-2,12-Diacetyl-7-methoxycarbonyl-tridec-4,9-diendisäure-dimethylester 285 .125

4.9.4. (E)-2,7,12-Trismethoxycarbonyl-tridec-4,9-diendisäure-dimethylester 286.......... 126

4.9.5. (E)-6-Hydroxy-2-(4-hydroxybut-2-enyl)-hexen-4-ensäure-methylester 288 .........126

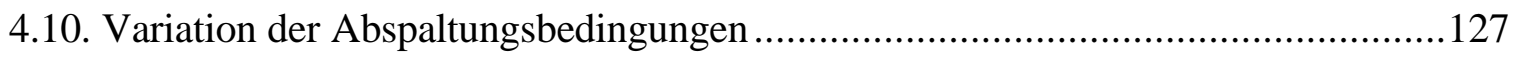

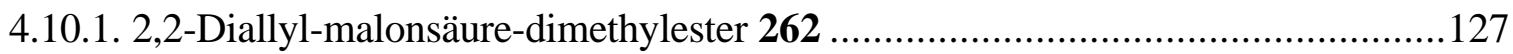

4.10.2. (E)-5-Phenyl-2-(3-phenylallyl)-pent-4-ensäure-methylester 263 ...................... 127

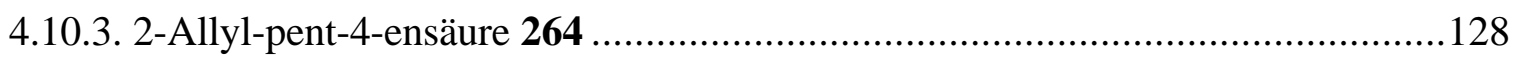

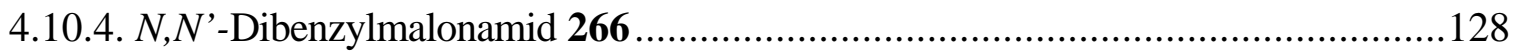

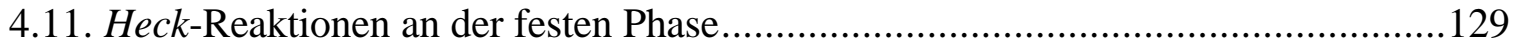

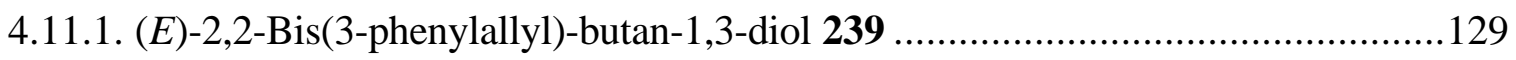

4.11.2. (E)-2,2-Bis(3-(3-methoxyphenyl)-allyl)-butan-1,3-diol 243 .............................129

4.11.3. (E)-2,2-Bis(3-(3-methoxyphenyl)-allyl)-propan-1,3-diol 302 ..........................129

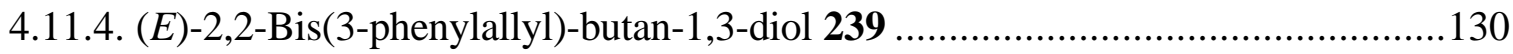

4.11.5. (E)-2,2-Bis(3-(3-methoxyphenyl)-allyl)-butan-1,3-diol 243 ............................ 131

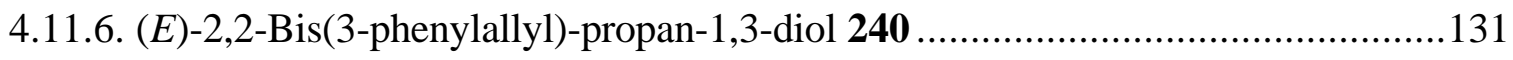

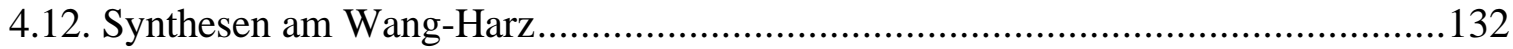

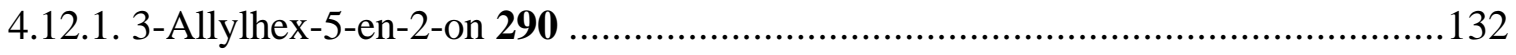

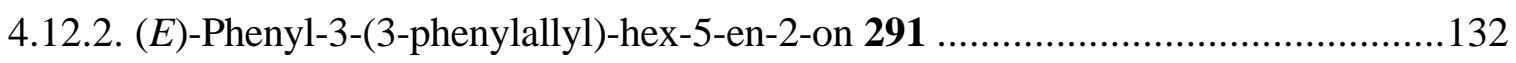

4.12.3. (E)-2,7,12-Triacetyl-tridec-4,9-diensäure-dimethylester 295 ..........................133 
5. Festphasensynthese von Pyrazolonen..................................................................134

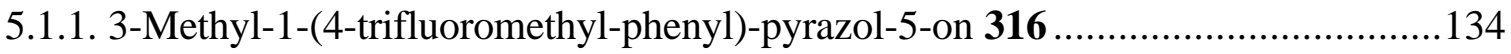

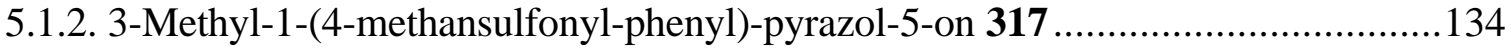

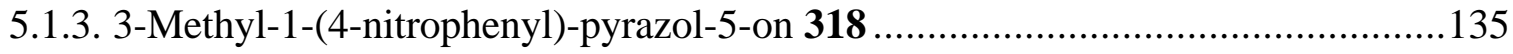

5.1.4. 1-(2-Chloro-6,7-dihydro-5H-cyclopentapyrimidin-4-yl)-3-methyl-pyrazol-

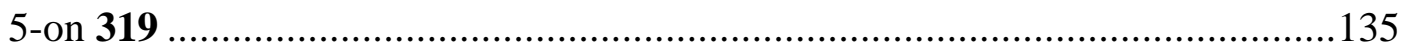

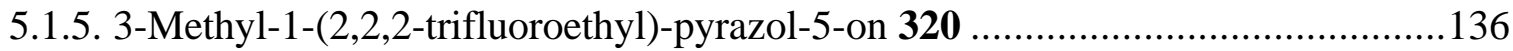

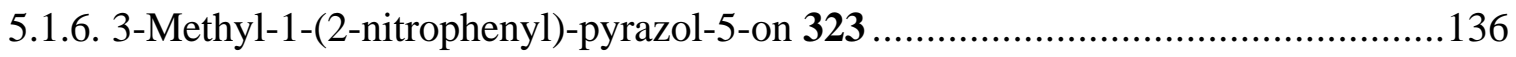

5.1.7. 1-(2,4-Difluorophenyl)-3-methyl-pyrazol-5-on 327 ....................................... 137

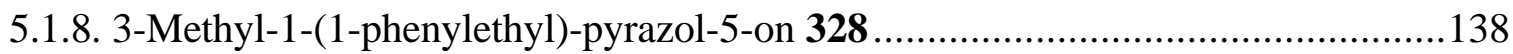

5.1.9. 3-Methyl-1-(2-methyl-cyclohexyl)-pyrazol-5-on 329.................................... 138

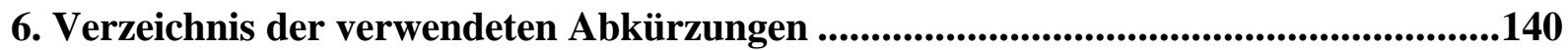

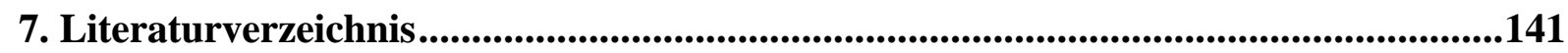

8. Danksagungen ...............................................................................................................146

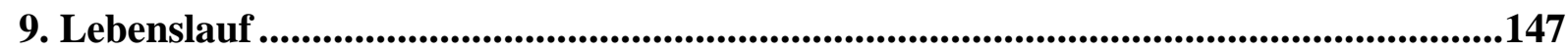




\section{A. Allgemeiner Teil}

\section{Einleitung}

Die chemische Forschung befindet sich in einem fortwährenden Wandel. Neue Entwicklungen und Entdeckungen veränderten ihr Erscheinungsbild, und die Grenze des Durchführbaren wurde immer wieder neu definiert. Während zu Beginn des Jahrhunderts die Konstitution der synthetisierten Verbindungen im Vordergrund stand, interessierte mehr und mehr auch deren Stereochemie. Die Etablierung der NMR-Spektroskopie als routinemäßige Labortechnik ermöglichte die immer genauere Untersuchung der Syntheseprodukte, was die Entwicklung diastereo- und enantioselektiver Methoden vorantrieb. Neben der Eleganz einer Synthese entstand aus ökonomischer und ökologischer Sicht das Interesse an umwelt- und ressourcenschonenden Umsetzungen. Lösungsansätze bestehen in der Verwendung sequentieller Transformationen ${ }^{[1]}$ und in katalytischen Prozessen. ${ }^{[2]}$ Intensive Forschung zur Verbesserung der Methodiken werden insbesondere in Forschungsgebieten durchgeführt, die sehr langwierig und daher kostenintensiv sind wie beispielsweise die Suche nach neuen pharmakologischen Wirkstoffen.

Bei der klassischen Wirkstoffsuche werden nach der Identifizierung einer Leitstruktur eine Vielzahl von strukturellen Variationen dieses Moleküls synthetisiert, um dessen Wirkungsprofil so zu verbessern und zu optimieren, daß am Ende ein Arzneistoff resultiert. In der Regel werden dazu viele tausend neue Verbindungen synthetisiert, charakterisiert und auf ihre biologische Aktivität getestet. ${ }^{[3]}$

Angetrieben durch Fortschritte in der Molekularbiologie und der Gentechnik ${ }^{[4]}$ gelingt die Identifizierung und Strukturaufklärung von Proteinen (Enzyme, Rezeptoren), die direkt mit den Krankheitsbefunden in Verbindung stehen. Dadurch eröffnet sich die Möglichkeit zum Verständnis der Ursachen von Krankheiten auf molekularer Ebene. Mit Hilfe solcher biologischer Targets konnten in den vergangenen Jahren eine Reihe neuer, hocheffizienter ScreeningVerfahren entwickelt werden, die durch Automatisierung und Miniaturisierung einen hohen Probendurchsatz ermöglichen. So können pro Jahr 100.000 Substanzen in einem Testsystem auf ihre biologische Wirkung hin untersucht werden. ${ }^{[5 a]}$ Eine effiziente Nutzung dieser sogenannten High-Throughput-Screening-Methoden ist nur dann gewährleistet, wenn in kurzen Zeiträumen eine große Anzahl strukturell verschiedener Testsubstanzen zur Verfügung gestellt werden. Dies kann von der traditionellen Synthesechemie nur schwer geleistet werden. Daher wurde die Kombinatorische Chemie ${ }^{[5]}$ entwickelt, die es erlaubt, in kurzer Zeit mittels weniger synthetischer Schritte eine Vielzahl diversomerer Verbindungen herzustellen. Ziel der Synthese ist hier nicht mehr die Herstellung einer einzelnen, genau definierten Struktur, sondern die pa- 
rallele, multiple Synthese von Substanzmischungen. Das Verfahren ermöglicht aber auch die effiziente parallele Synthese von Einzelsubstanzen. Die Mehrzahl der kombinatorischen Synthesen werden an der festen Phase durchgeführt, wodurch ein hohes Maß an Automatisierung möglich ist.

Die Kombinatorische Chemie greift an zwei Stellen in den Ablauf der Wirkstoffentwicklung ein, bei der Suche nach Leitstrukturen und bei ihrer Optimierung. Für ersteren Zweck werden sogenannte Bibliotheken benötigt, die aus einer großen Anzahl von Verbindungen mit einem hohen Maß an Diversität bestehen. Bibliotheken hingegen, die bei der Optimierung einer Leitstruktur Verwendung finden, besitzen eine vergleichsweise niedrigere Diversität. Eine häufig eingesetzte Strategie besteht in der Untersuchung von pharmakologisch aktiven Substanzklassen, die bereits Arzneimittel hervorgebracht haben wie z. B. die 1,4-Benzodiazepine und die Pyrazolone (siehe Kapitel 8). Obwohl sich beide Leitstrukturen schon lange im Blickfeld der Pharmaforschung befinden, eröffnet die Synthese vieler neuer Derivate durch kombinatorische Methoden neue Möglichkeiten in der Wirkstoffentwicklung. 


\section{Synthesen an der festen Phase}

\subsection{Allgemeine Prinzipien}

Anfang der 60er Jahre berichtete B. Merrifield ${ }^{[6]}$ über chemische Transformationen von Substraten, die an ein polymeres Trägermaterial gebunden waren. Er entwickelte eine automatisierbare Methode zur effizienten Synthese von Peptiden und wurde dafür 1984 mit dem Nobelpreis ausgezeichnet. Dieses revolutionäre Verfahren wird heute in vollautomatischen Syntheseautomaten zum Aufbau von Peptiden und Oligonucleotiden verwendet. ${ }^{[7]}$ Die Synthese kleiner, organischer Verbindungen am polymeren Träger hingegen genoß lange Zeit nur geringes Interesse und wurde in den 70er und frühen 80er Jahren im wesentlichen von J. Fréchet ${ }^{[8]}$ und C. Leznoff ${ }^{[9]}$ durchgeführt. Dies änderte sich Anfang der 90er Jahre schlagartig, als die Festphasensynthese durch ihre Nutzung im Rahmen der Kombinatorischen Chemie (siehe Kapitel 2.3.) eine schwunghafte Renaissance erlebte.

Zur Durchführung von Synthesen an der festen Phase wird das Reaktionsedukt über einen sogenannten Linker kovalent an das polymere Trägermaterial gebunden. Die nachfolgenden Reaktionen können unter Verwendung großer Reagenzüberschüsse durchgeführt werden, was die Produktbildung in hohen Ausbeuten begünstigt (siehe Abbildung 1). Nach beendeter Umsetzung kann das unlösliche Polymer und das daran kovalent gebundene Reaktionsprodukt durch Filtration von der flüssigen Phase und den darin gelösten Reagenzien und möglichen Nebenprodukten abgetrennt werden. Zeitaufwendige Reinigungen des Reaktionsprodukts durch chromatographische oder destillative Prozesse werden durch einfaches Waschen des Harzes ersetzt. Auf diese Weise können die Produkte einer mehrstufiger Synthesesequenz nach Abspaltung vom polymeren Träger in hohen Reinheiten erhalten werden. Diese genügen im allgemeinen den Ansprüchen moderner Screening-Verfahren, mit denen die erhaltenen Substanzen auf ihre biologische Aktivität hin untersucht werden können. Die Durchführung von Synthesen an der festen Phase läßt sich leicht automatisieren und bietet sich daher zum Einsatz in der multiplen, parallelen Herstellung von Einzelverbindungen und Verbindungsbibliotheken im Sinne der Kombinatorischen Chemie an. 


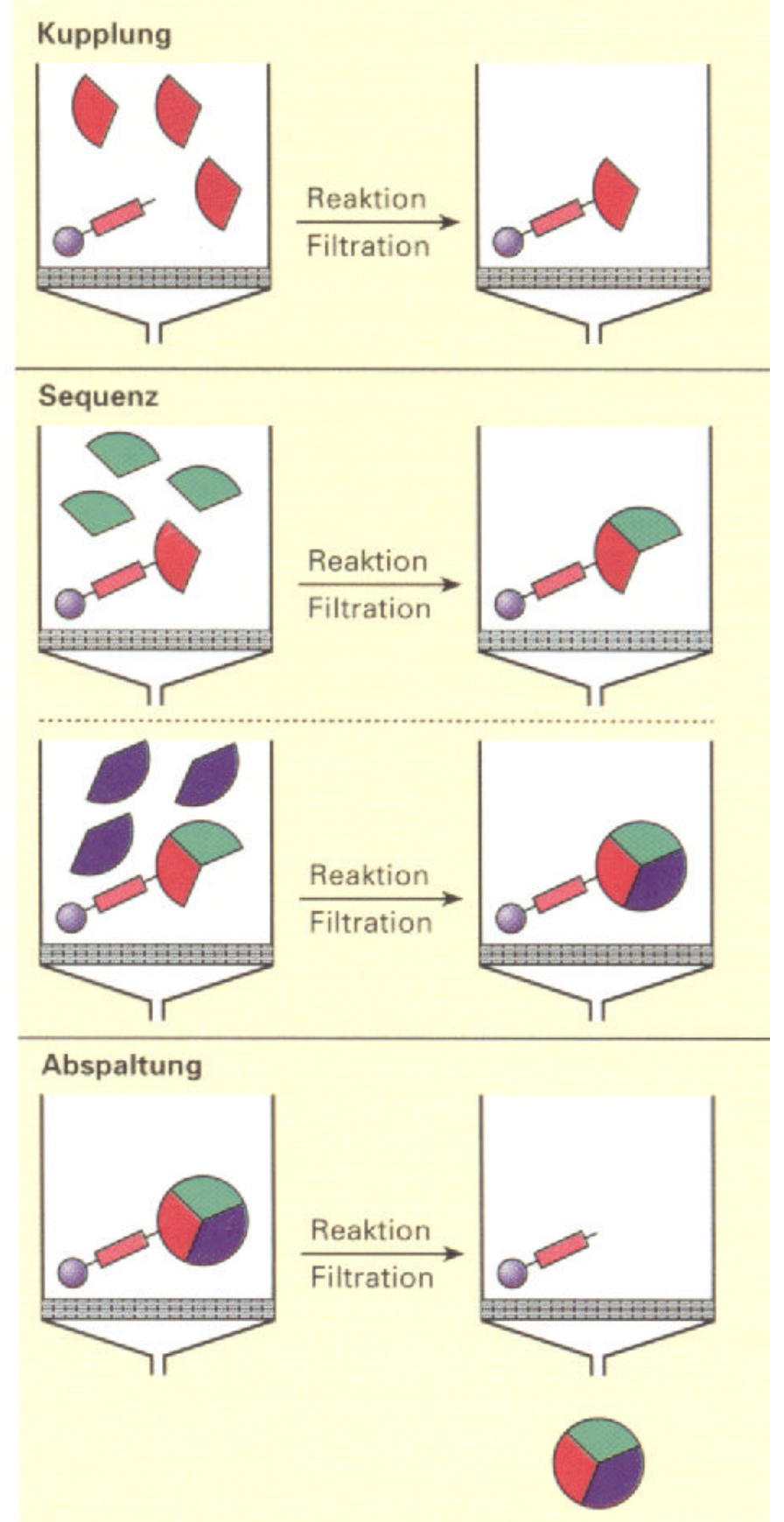

Abbildung 1: Syntheseführung an der festen Phase.

Zu den Nachteilen der Festphasensynthese zählt die Erhöhung der Reaktionschritte durch den zusätzlichen Anknüpfungs- und Abspaltungsschritt. Dank entsprechend entwickelter Linker verlaufen diese Transformationen jedoch meist nahezu quantitativ. Ein weiteres Problem besteht in den eingeschränkten Möglichkeiten der spektroskopischen Charakterisierung polymergebundener Verbindungen. Die in Lösung routinemäßig durchgeführte NMR-Spektroskopie liefert aufgrund starker Signalverbreiterungen durch die gehinderte Beweglichkeit der immobi- 
lisierten Substrate kaum verwertbare Ergebnisse. ${ }^{[10]}$ Neuere Entwicklungen, wie die MASTechniken („magic angle spinning“) und die Verwendung einer sogenannten Nano-ProbeSonde versprechen Abhilfe. ${ }^{[11]}$ Wegen der fehlenden Flüchtigkeit lassen sich polymergebundene Verbindungen auch nicht mit herkömmlichen Methoden massenspektroskopisch untersuchen. Spezielle Techniken wie ESI-MS, MALDI/TOF-MS und TOF/SIMS jedoch ermöglichen es, Massenspektren von Substanzen zu erhalten, die an einzelne Harzpartikel gebunden sind. ${ }^{[12]}$ Sehr einfach und schnell läßt sich die IR-Spektroskopie durchführen. Da aber bereits der polymere Träger ein sehr bandenreiches IR-Spektrum besitzt, werden viele Schwingungen der organischen Verbindung überlagert. Die meisten Polymere besitzen jedoch Bereiche geringer Absorption, so daß für die Reaktionskontrolle an den häufig verwendeten Polystyrol-Harzen z. B. die Banden von Carbonyl-Gruppen geeignet sind.

\subsection{Harze und Linker}

Als feste Phase dienen meist Polystyrol-Harze, aber auch Polyethylen, Hexamethylendiaminpolyacryl sowie Kieselgur und poröse Glasperlen kommen zum Einsatz. ${ }^{[13]}$ Da sich Polystyrol in vielen organischen Solventien löst, wird ein mit 1-2\% Divinylbenzol querverknüpftes Copolymer verwendet, das auch bessere mechanische Eigenschaften besitzt. Der polymere Träger enthält sogenannte Linker, welche die Anknüpfung und spätere Abspaltung von organischen Verbindungen ermöglichen (siehe Abbildung 2). Neben dem chlormethylierten Merrifield-Harz 1 und dem mit langen Polyethylenglycol-Ketten versehenen Tentagel-Harz $\mathbf{2}$ kommen besonders das Wang-Harz 3 mit einer terminalen Hydroxy-Gruppe sowie das Rink-Amid-Harz $\mathbf{5}$ mit einer Amino-Funktionalität zum Einsatz. Das Merrifield-Harz 1 ist dabei in der Anschaffung das kostengünstigste Harz und kann nach einer im Arbeitskreis L. F. Tietze ${ }^{[14]}$ entwickelten Methode mit einem sogenannten Spacer versehen werden, der eine gute Reaktivität der nachfolgend an das Polymer gebundenen Substrate gewährleistet. ${ }^{[14 a]}$ Die Konzentration der Anknüpfungsstellen der Harze und damit die Beladungsdichte polymergebundener Verbindungen variiert zwischen 0.1 und $1.2 \mathrm{mmol}$ pro Gramm Harz; das Merrifield-Harz 1 ist auch mit Beladungskapazitäten von bis zu 5 mmol pro Gramm erhältlich. ${ }^{[15]}$ 


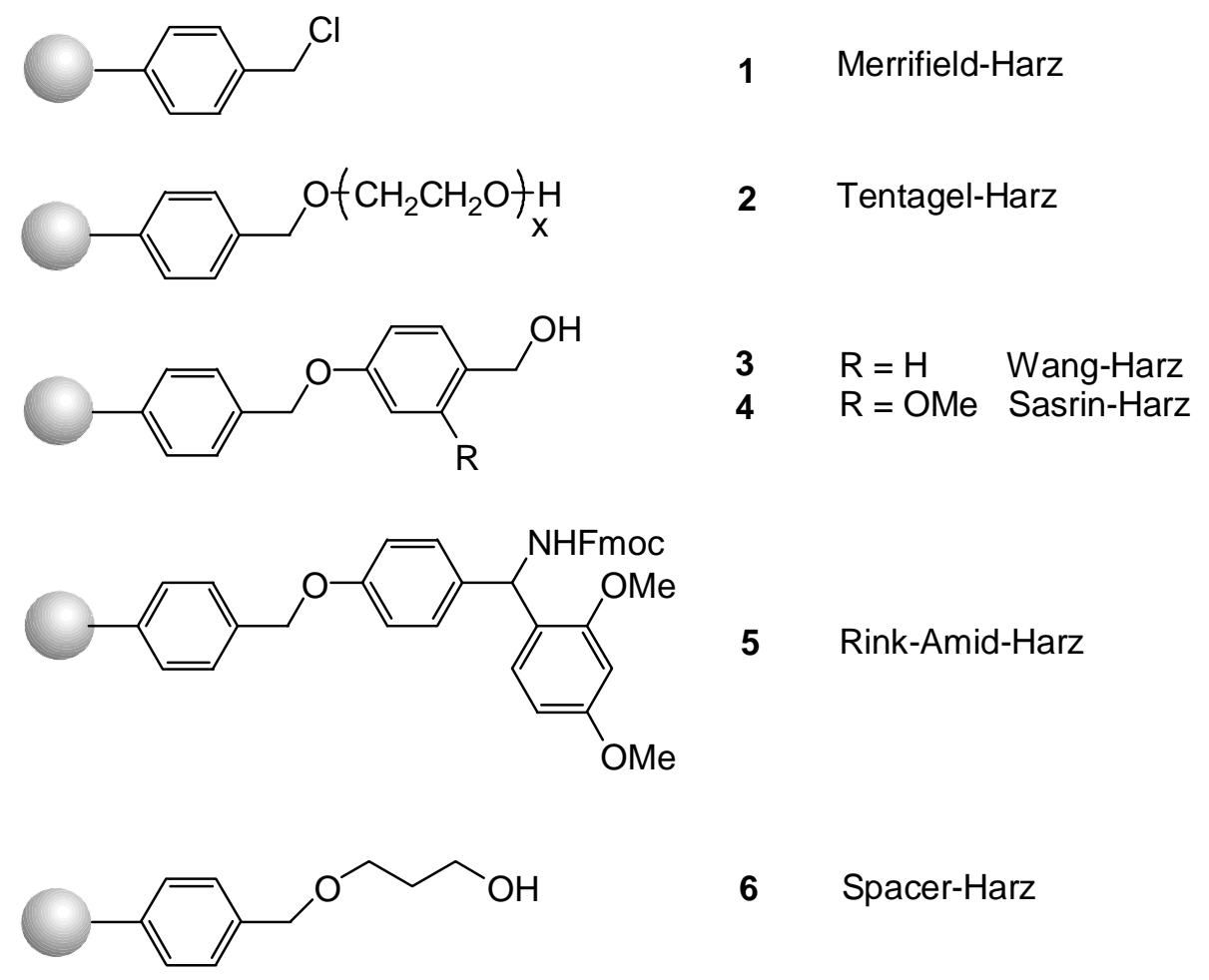

Abbildung 2: Verschiedene Harze für die Festphasensynthese.

Polystyrol-Harze, die meist in Pulverform mit einer Partikelgröße von 40-63 $\mu \mathrm{m}$ (200400 mesh) erhältlich sind, besitzen die Fähigkeit, in unpolaren Lösungsmitteln wie Dichlormethan, Toluol und Tetrahydrofuran um ein Mehrfaches des Trockenvolumens zu quellen. Da sich die eingeführten Funktionalitäten nicht nur an der Oberfläche der Polymere, sondern hauptsächlich im Inneren der Harz-Partikel befinden, ist es außerordentlich wichtig, Lösungsmittel mit guten Quelleigenschaften zu verwenden. Andernfalls können die Reagenzien nicht in das Harz diffundieren und die polymergebundenen Substrate erreichen, so daß unvollständige Umsetzungen resultieren. Polare Lösungsmittel wie Alkohole und Wasser, in denen das Harz nur schlecht quillt oder sogar wie im Falle von Wasser verklumpt, sind daher wenig geeignet für Festphasenreaktionen an Polystyrol-Harzen. Hier kann jedoch auf andere Trägermaterialien ausgewichen werden, wie z. B. Polyethylenglykol-Harze, die in polaren, nicht aber in unpolaren Lösungsmitteln quellen. Auch das Tentagel-Harz 2 zeigt gute Quelleigenschaften in polaren Solventien, doch der vergleichsweise hohe Preis und die sehr geringe Beladungsdichte von 0.1 - $0.3 \mathrm{mmol} / \mathrm{g}$ schränken die Benutzung ein.

Bei einer durchschnittlichen Beladungsdichte von $0.8 \mathrm{mmol} / \mathrm{g}$ sind ungefähr $5 \%$ der Phenylringe des Polystyrol-Grundgerüstes funktionalisiert. Dies bedeutet, daß die einzelnen polymergebundenen Substrate räumlich so weit voneinander getrennt sind als ob sie sich in einer hochverdünnten Lösung befänden (Pseudoverdünnungseffekt). ${ }^{[5,14,16]}$ Dies ermöglicht z. B. intramolekulare Ringschlußreaktionen oder die einseitige Umsetzung bifunktioneller Moleküle, die 
unter vergleichbaren Bedingungen in Lösung nicht realisierbar wären. Ein anderer Verdünnungseffekt beruht auf der Tatsache, daß die Reagenzien in Lösung zunächst in das Polymer hineindiffundieren müssen, um mit dem festphasengebundenem Substrat zu reagieren. Durch diese Diffusion ist die tatsächliche Konzentration des Reagenz am Reaktionsort deutlich geringer als in der übrigen Reaktionsmischung. ${ }^{[17]}$

Der polymere Träger ist mit einem Linker ${ }^{[18]}$ versehen, der die Brücke zwischen dem polymeren Grundkörper und dem Substrat darstellt. Er besitzt eine terminale Funktionalität, die einerseits das leichte Anknüpfen organischer Verbindungen, andererseits deren milde und quantitative Abspaltung nach beendeter Reaktionssequenz gewährleistet. Während der Reaktionen an der festen Phase hingegen muß er inert sein (siehe Abbildung 3). Dies erinnert an das Konzept einer Schutzgruppe, die sich ebenfalls durch leichte Anknüpfung und Abspaltung sowie Stabilität während der Transformationen auszeichnen muß.
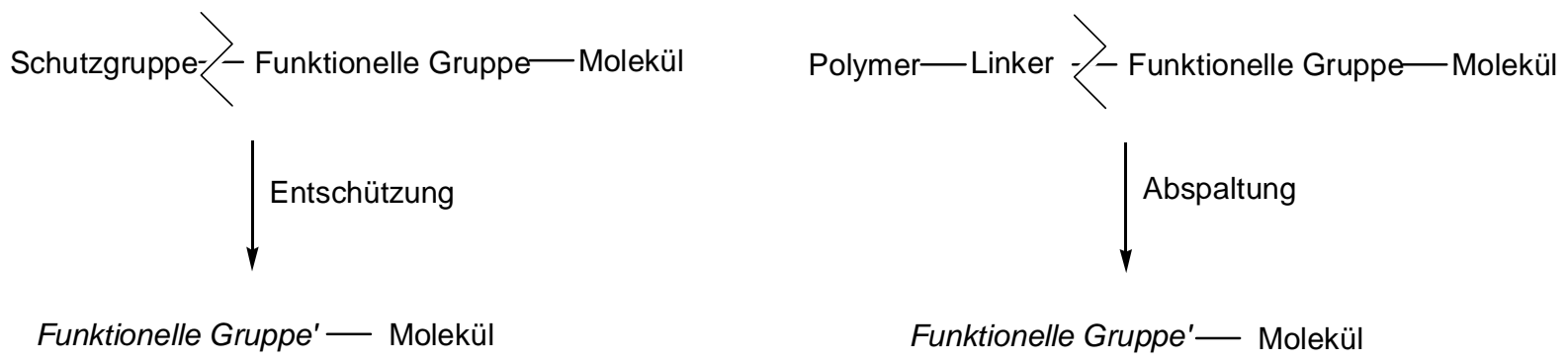

Abbildung 3: Prinzip eines Linkers.

Wie die meisten Harze stammen viele der verwendeten Linker aus der Peptidchemie. Dies führt allerdings häufig zu methodischen Einschränkungen. Die Abspaltung erfolgt meist unter sauren Bedingungen und hinterläßt in der Zielverbindung üblicherweise funktionelle Gruppen wie Carbonsäuren oder Carbonsäureamide, die eventuell an dieser Stelle unerwünscht sind. Es wurden jedoch eine Reihe neuerer Linker speziell für die organische Festphasensynthese entwickelt.

J. A. Ellman ${ }^{[19]}$ stellte den Linker 7 vor, der als eine festphasengebundene THP-Schutzgruppe betrachtet werden kann. Unter Säurekatalyse lassen sich primäre und sekundäre Alkohole an den polymeren Träger binden. Die Abspaltung erfolgt mit Trifluoressigsäure unter Freisetzung des Alkohols.

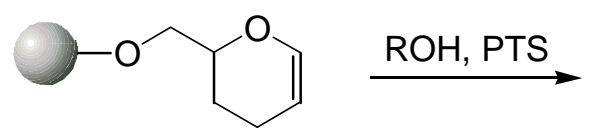

7

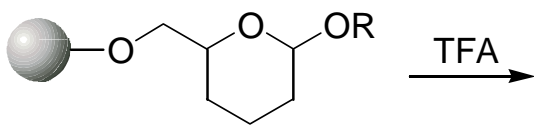

8
$\mathrm{ROH}$

9

Abbildung 4: THP-Linker. 
Linker, die gegenüber einer Vielzahl von Reaktionsbedingungen stabil sind, nach Aktivierung jedoch unter sehr milden Bedingungen die Abspaltung der Zielverbindung zulassen, sind für die organische Festphasensynthese von großem Interesse. G. W. Kenner ${ }^{[20]}$ stellte einen derartigen „Safty-catch“-Linker vor, indem er das polymergebundene Sulfonamid $\mathbf{1 0}$ mit aktivierten Carbonsäurederivaten umsetzte. Das erhaltene Acylsulfonamid 11 ist inert gegenüber basischen und nucleophilen Reagenzien. Nach Aktivierung durch Alkylierung des SulfonamidStickstoffes mit Iodmethan oder Iodacetonitril läßt sich die Abspaltung unter milden Bedingungen mit verschiedenen primären und sekundären Aminen sowie Hydroxid-Ionen erreichen.

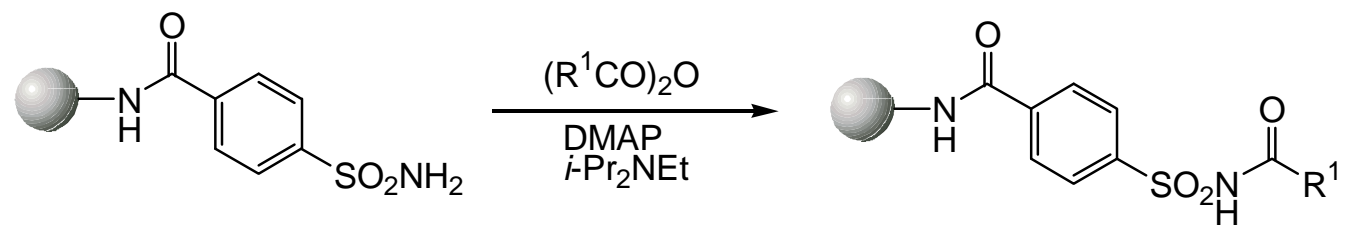

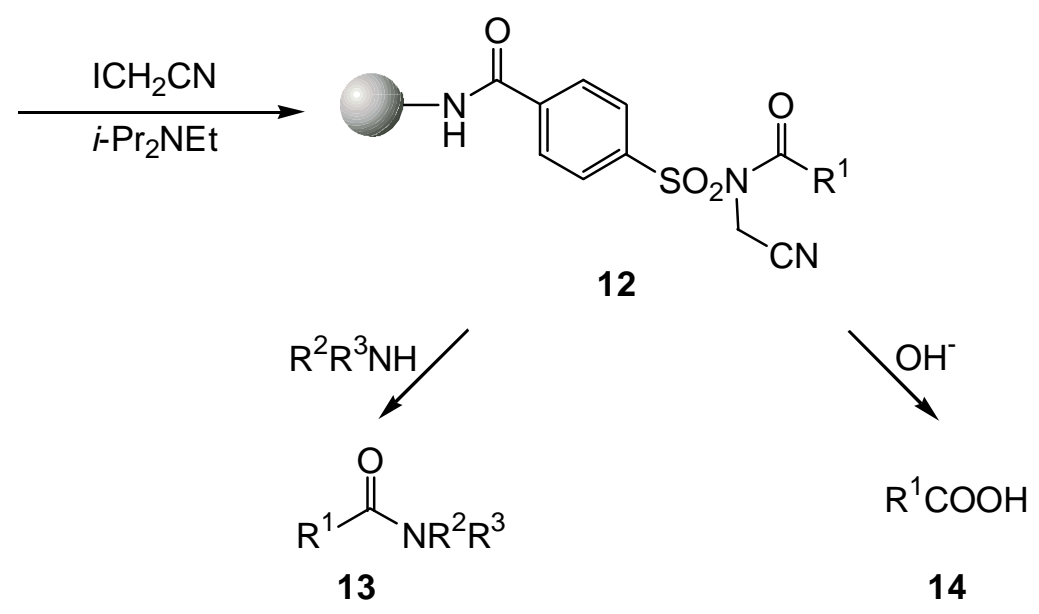

Abbildung 5: Safty-Catch-Linker.

Üblicherweise verbleibt nach erfolgter Abspaltung von der festen Phase an der Anknüpfposition des Zielmoleküls eine funktionelle Gruppe zurück, die jedoch manchmal unerwünscht sein kann. Durch Verwendung sogenannter ,traceless“ Linker erhält man an dieser Stelle eine C-HBindung. J. A. Ellman ${ }^{[21]}$ zeigte dies mit dem Silyl-Linker 15, der mit Fluoriden gespalten werden kann. Neben Silizium kommen auch Germanium, Selen und Phoshor als Heteroatome zum Einsatz. ${ }^{[22]}$ 


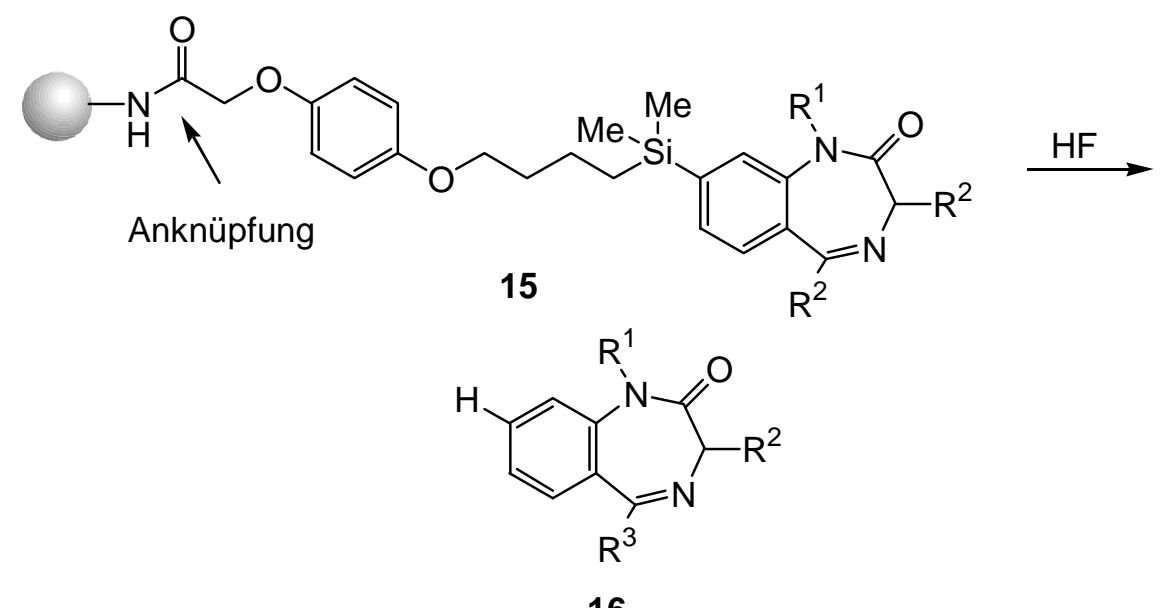

Abbildung 6: „Traceless“ Linker.

Eine weitere Strategie, um unerwünschte Funktionalitäten in der Zielverbindung zu vermeiden, besteht in der Abspaltung im letzten Schritt der Synthesesequenz. Dies gelingt z. B. durch Cyclisierungen unter gleichzeitiger Freisetzung vom polymeren Träger. Beispiele hierfür werden in Kapitel 2.4. besprochen.

Bei allen bisher beschriebenen Abspaltungsmethoden erfolgt die Freisetzung der polymergebundenen Zielverbindung durch Zugabe eines Abspaltungsreagenzes. Eine andere Möglichkeit ist die Verwendung von photolabilen Linkern, bei denen die Abspaltung durch Bestrahlung mit Licht einer bestimmten Längenwelle erreicht wird. C. P. Holmes ${ }^{[23]}$ demonstrierte dies bei der Synthese des 4-Thiazolidinons 18. Photochemische Abspaltungsmethoden stellen eine interessante Alternative dar, wenn übliche Abspaltungsmethoden nicht mit dem Produkt kompatibel sind. Auf die Festphasensynthese einer 4-Thiazolidinon-Bibliothek wird in Kapitel 2.4. eingegangen.<smiles>COc1cc(C(C)NC(=O)CN2C(=O)CSC2c2ccccc2)c([N+](=O)[O-])cc1OCCCC(=O)Nc1ccccc1</smiles><smiles>NC(=O)CN1C(=O)CSC1c1ccccc1</smiles>

18

Abbildung 7: Photolabile Linker. 


\subsection{Kombinatorische Chemie}

Der Denkansatz der Kombinatorischen Chemie ${ }^{[5,17,24]}$ erscheint für einen Chemiker auf den ersten Blick ungewohnt. Entgegen der traditionellen Vorgehensweise, jeweils nur eine einzelne, wohldefinierte Struktur zu einer Zeit herzustellen, geht es um die gleichzeitige Darstellung einer Vielzahl von chemisch ähnlichen -diversomeren- Verbindungen, die in Form einer Substanzbibliothek erhalten werden. Diese Substanzbibliotheken können dabei aus Verbindungsgemischen oder aus einer Ansammlung von Einzelverbindungen bestehen. Statt zwei Edukte A und $\mathbf{B}$ zu einem Produkt AB zu verknüpfen, werden mehrere Synthesebausteine des Typs A $\left(\mathbf{A}_{1-\mathbf{n}}\right)$ mit mehreren des Typs $\mathbf{B}\left(\mathbf{B}_{\mathbf{1 - n}}\right)$ umgesetzt, so daß man alle möglichen Kombinationen erhält (siehe Abbildung 8). Dieses kann parallel in getrennten Gefäßen oder simultan in einer Mischung erfolgen. Verwendet man fünf Bausteine A und fünf Bausteine $\mathbf{B}$, so erhält man in einem Syntheseschritt 25 neue Verbindungen. Bei jedem weiteren Schritt potenziert sich die Zahl und nach weiterer Umsetzung mit fünf Bausteinen $\mathbf{C}$ hätte man bereits 125 diversomere Strukturen. Mathematisch läßt sich dieser exponentielle Zuwachs durch $\mathrm{x}^{\mathrm{n}}$ beschreiben, wobei $\mathrm{x}$ die Anzahl der verwendeten Synthesebausteine und n die Anzahl der ausgeführten Reaktionsschritte angibt.
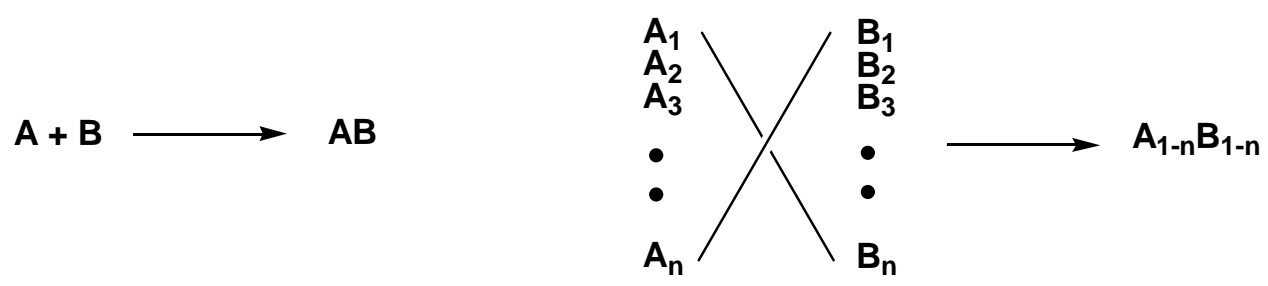

\section{Abbildung 8: Synthesestrategie.}

Die kombinatorischen Synthesemethoden können sowohl in Lösung als auch am polymeren Träger durchgeführt werden. Letzteres ist die in den meisten Fällen bevorzugte Variante, um die durch die Synthese an der festen Phase entstehenden Vorteile zu nutzen (siehe Kapitel 2.1.). Dennoch gibt es eine Reihe interessanter Anwendungen und Beispiele für die Generierung kombinatorischer Bibliotheken in flüssiger Phase. ${ }^{[25]}$

Die Reaktionsdurchführung an der festen Phase ist hervorragend geeignet, Substanzmischungen in einer parallelen Synthesestrategie durch ein ,split and combine“-Protokoll ${ }^{[26]}$ aufzubauen (siehe Abbildung 9). Dabei wird der polymere Träger in gleich großen Portionen auf verschiedene Gefäße verteilt und jeweils mit einem einzelnen Reagenz umgesetzt. Nach der Reaktion werden die Harze gründlich durchmischt und erneut nach gleichmäßiger Aufteilung in Einzelreaktionen eingesetzt. Nach beliebig häufiger Wiederholung dieses Vorganges werden alle möglichen Kombinationen der Synthesebausteine erhalten. Die diversomeren Verbindungen 
werden dabei in äquimolaren Mengen gebildet, wobei jedes Harz-Kügelchen nur eine, definierte Verbindung trägt (,one bead - one compound“).

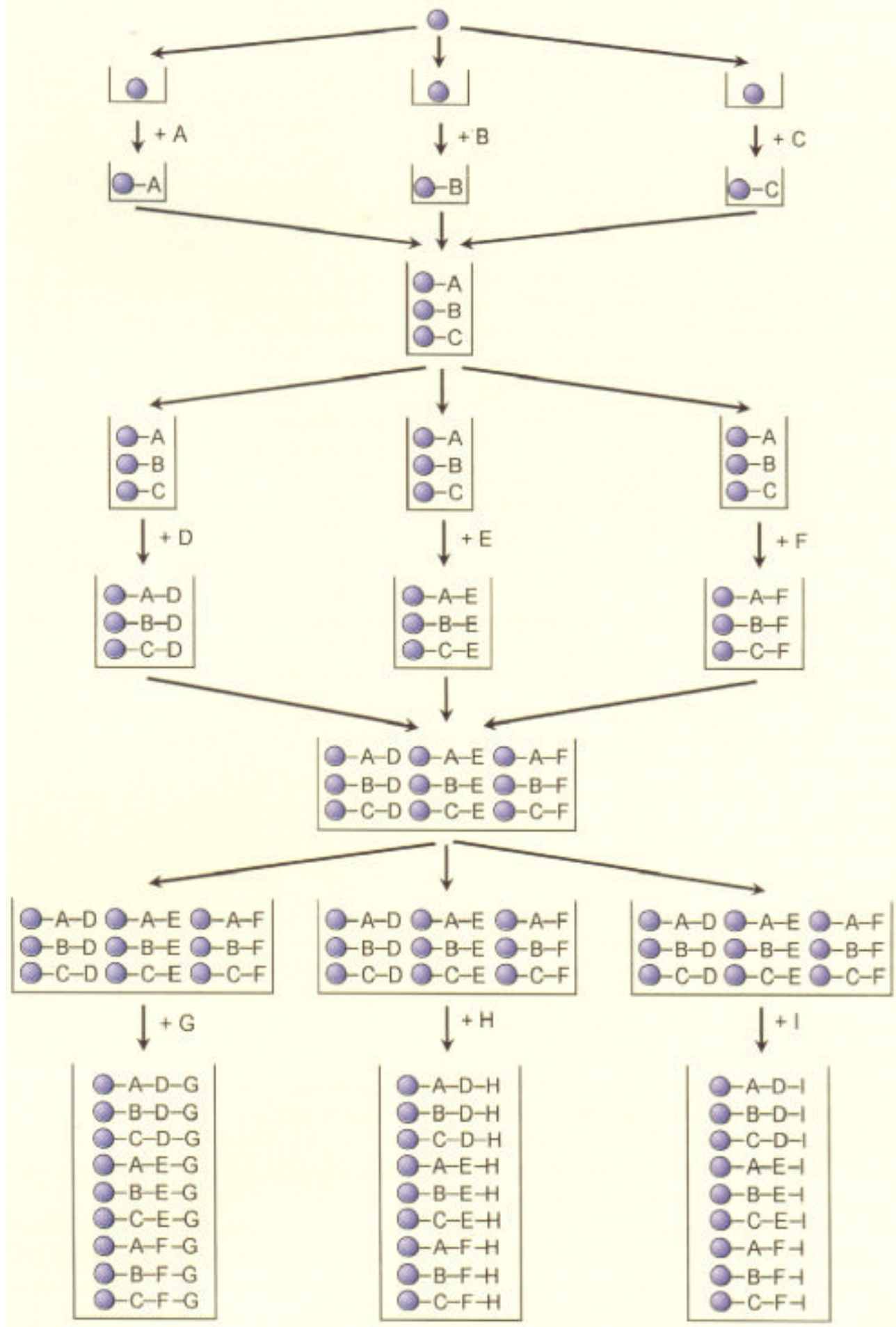

Abbildung 9: ,Split and combine“-Protokoll. 
Beim Aufbau einer Substanzbibliothek mittels simultaner Reaktionsführung werden die Synthesebausteine jeder Stufe als Mischung eingesetzt. Dadurch konkurrieren sie miteinander, und die Produkte werden in Abhängigkeit von der Reaktivität der Reagenzien in unterschiedlichen Anteilen gebildet. Diese Strategie mindert den Arbeitsaufwand durch die Verwendung lediglich eines Reaktionsgefäßes enorm, sollte jedoch nur benutzt werden, wenn sich die Synthesebausteine in ihrem Reaktionsverhalten nicht allzu stark unterscheiden.

Dank des hohen Entwicklungsstandes von halb- bzw. vollautomatischen Syntheseautomaten ${ }^{[27]}$ gewinnt der Aufbau von kombinatorischen Substanzbibliotheken durch parallele Synthese von Einzelverbindungen immer mehr an Bedeutung. Dies gilt besonders für die der Anwendung kombinatorischer Methoden bei der Suche und Optimierung von Wirkstoffen. Es hat sich als günstiger herausgestellt, einzelne Verbindungen auf ihre biologische Aktivität hin zu testen als dies in Mischung mit anderen Substanzen vorzunehmen. ${ }^{[28]}$ Dadurch entfallen Probleme wie die Identifizierung der aktiven Komponente in der Mischung durch aufwendige Dekonvolutionstechniken, d. h. schrittweise Resynthese von Subbibliotheken bis zur Einzelverbindung. ${ }^{[24]}$ Auch werden verfälschte Testergebnisse durch Synergieeffekte vermieden. Ferner ist die Anzahl der Verbindungen, die pro Zeiteinheit untersucht werden können, durch fortschreitende Optimierungen und Automatisierungen der Screening-Verfahren in den letzten Jahren derart gestiegen, daß ausreichende Kapazitäten zur Verfügung stehen. ${ }^{[28]}$

Bei der kombinatorischen Synthese von Substanzbibliotheken kommt es weniger auf eine überaus große Anzahl von Verbindungen an. Stattdessen ist viel mehr die Qualität der Bibliothek von entscheidender Bedeutung. Hierbei spielt der Begriff der Diversität eine wichtige Rolle. Eine Bibliothek ist divers, wenn ihre einzelnen Komponenten möglichst verschiedenartig sind. Dies bezieht sich im allgemeinen auf die räumliche Anordnung von funktionellen Gruppen, die für die nicht-kovalenten Wechselwirkungen mit den Test-Targets verantwortlich sind. Dabei werden für die Leitstruktursuche Bibliotheken mit einer hohen Diversität verwendet, für die Leitstrukturoptimierung dagegen werden Bibliotheken mit geringerer Diversität synthetisiert.

\subsection{Beispiele kombinatorischer Festphasensynthese}

Heterocyclen sind aufgrund ihrer oft vielfältigen biologischen Aktivitäten von großer Bedeutung für die Wirkstofforschung. Es ist daher verständlich, daß ein Großteil der kombinatorischen Festphasensynthesen auf heterocyclische Verbindungen konzentriert ist. Dabei werden vielfach aus der Lösungschemie bekannte Synthesen an die feste Phase übertragen. Obwohl die so erhaltenen Verbindungsklassen bereits bekannt und zum Teil gut erforscht sind, bietet die kombinatorische Synthese den Zugang zu einer Fülle von Variationen der Grundstrukturen, die für das Screening interessant sind. 
J. A. Ellman ${ }^{[29]}$ verwendete als Schlüsselschritt seiner Heterocyclensynthese eine StilleKupplung, mit der aus polymergebundenen N-Bpoc-geschützten 2-Aminoarylstannanen 19 und Carbonsäurechloriden 20 2-Aminoarylketone 21 an der festen Phase synthetisiert wurden. Durch Umsetzung mit $\alpha$-N-Fmoc-Aminosäurefluoriden 22 und anschließender Cyclisierung erhielt er den polymergebundenen Benzodiazepin-Grundkörper 24. Alkylierung mit 25 und Abspaltung mit TFA lieferte die diversomeren Benzodiazepine 26 in Ausbeuten zwischen 46 und $72 \%$ mit Reinheiten größer 85\%. Durch die Verwendung von einfachen, kommerziell in großem Umfang erhältlichen Synthesebausteinen (Carbonsäurechloride, Aminosäuren, Alkylierungsmittel) konnte eine Bibliothek von 11200 diversomeren Benzodiazepinen aufgebaut werden. $^{[24 a]}$

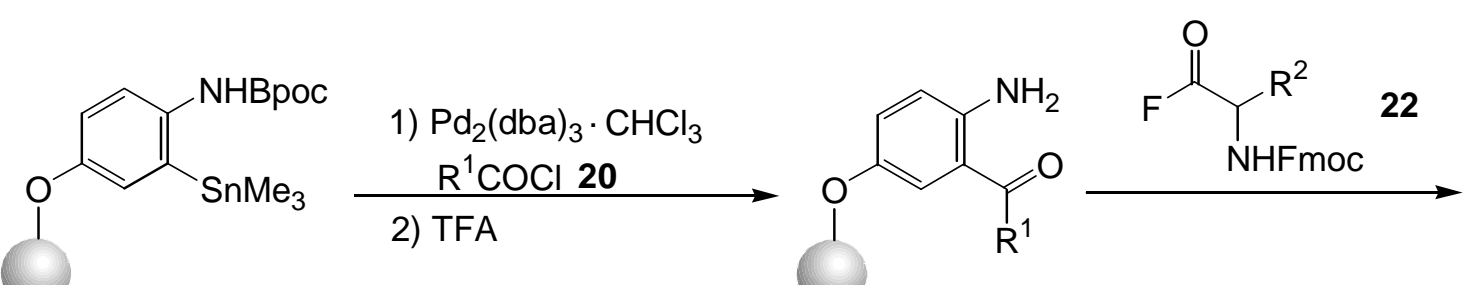

19

21<smiles>[R7]C(=O)c1cc(OC2CCCC2)ccc1NC(=O)C(NCC)C(=O)O</smiles>

23<smiles>[R]C1=NC([R])C(=O)Nc2ccc(OC(C)C)cc21</smiles>

24

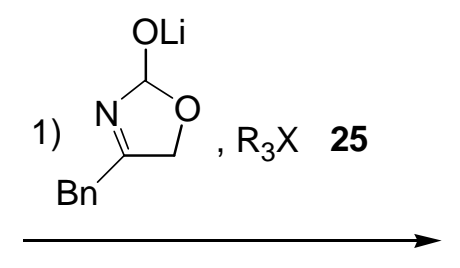

2) TFA, $\mathrm{CH}_{2} \mathrm{Cl}_{2}$<smiles>[R]C1=NC([R])C(=O)N([R])c2ccc(O)cc21</smiles>

26

Abbildung 10: 1,4-Benzodiazepine nach J. A. Ellman.

Eine kombinatorische Bibliothek von 4-Thiazolidinonen 29, einer Klasse von Cyclooxogenase1-Hemmern, beschrieb die Firma Affymax. ${ }^{[30]}$ Ausgehend von polymergebundenen Aminosäuren 27 wurden durch Kondensation mit Aldehyden die Imine 28 erhalten, welche durch Umsetzung mit Sulfanylessigsäure-Derivaten die 4-Thiazolidinone 29 lieferten. In Abhängigkeit von 
dem eingesetzten Linker konnten die Produkte als Amide 30 oder als Säuren abgespalten werden, die mit Diazomethan in die entsprechenden Methylester 31 überführt wurden. Mittels eines ,split and combine“-Protokolls wurden drei 4-Thiazolidinon-Bibliotheken mit jeweils 125 Mitgliedern synthetisiert. Die biologischen Testungen führten zur Identifizierung eines Inhibitors, der eine vergleichbare Wirkung wie die kommerziellen Cyclooxogenase-1-Hemmer Ibuprofen und Phenylbutazon ${ }^{[31]}$ aufweist.

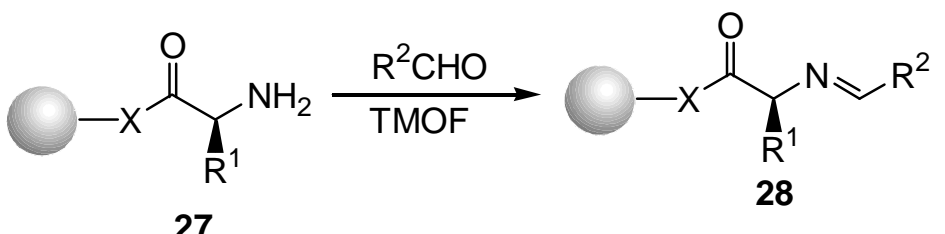<smiles>[X]C(=O)[C@H]([R7])N1C(=O)C([R])SC1[R7]</smiles>

29

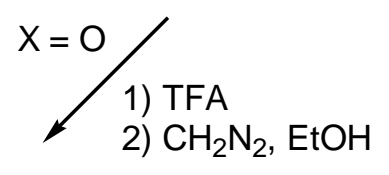

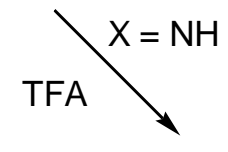<smiles>[R]C1SC([R7])N([C@@H]([R])C(=O)OC)C1=O</smiles>

30<smiles>[R]C1SC([R7])N([C@H]([R7])C(N)=O)C1=O</smiles>

31

Abbildung 11: 4-Thiazolidinone nach C. P. Holmes.

Neben relativ kleinen heterocyclischen Systemen wurden auch große Naturstoffe an der festen Phase aufgebaut. ${ }^{[32]}$ Diese Synthesen sind eng mit dem Namen K. C. Nicolaou verbunden, der eine Reihe von Naturstoffen am polymeren Träger generierte. So gelang ihm z. B. die Übertragung der von ihm in Lösung entwickelten Strategie zum Aufbau von diversen Epothilonen A an die feste Phase. ${ }^{[33]}$ Die Umsetzung des polymergebundenen Phosphorans 32 mit Baustein 33 lieferte in einer Wittig-Reaktion die Verbindung 34, welche nach Entschützung und Oxidation des Alkohols mit 35 gekuppelt wurde. Das Produkt dieser unselektiven Reaktion wurde mit 37 verestert. Die Abspaltung des Zielprodukts erfolgte unter Ringschluß durch eine MetatheseReaktion unter Ausbildung von 40, das in zwei Schritten in Lösung in Epothilon A 41 über- 
führt werden konnte. Dies ist ein Beispiel einer cyclisierenden Abspaltung, bei der der letzte synthetische Schritt und die Abpaltung von der festen Phase gleichzeitig geschehen.

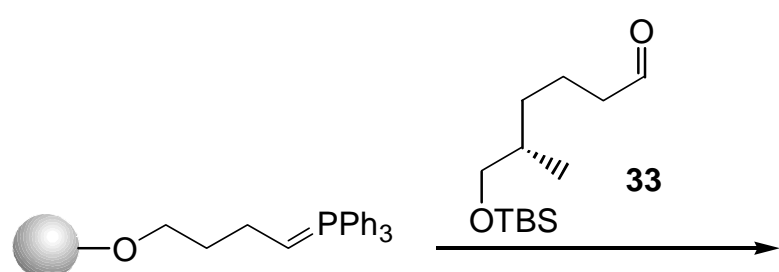

32

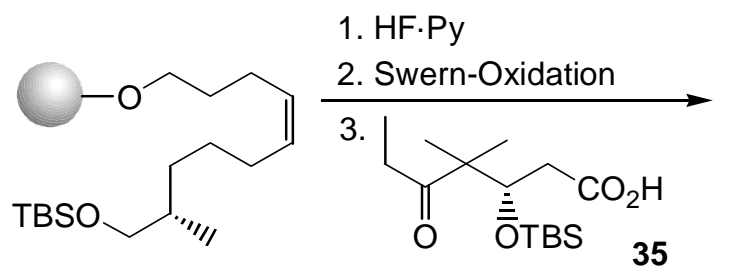

34

LDA, $\mathrm{ZnCl}_{2}$<smiles>C=CC[C@H](OC(=O)C[C@@H]([OH2+])C(C)(C)C(=O)C(C)[C@@H](O)[C@@H](C)CCCC/C=C\CCOC1CCCCC1)/C(C)=C/c1csc(C)n1</smiles>

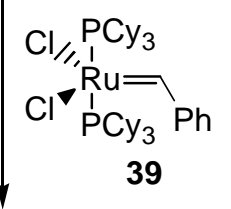

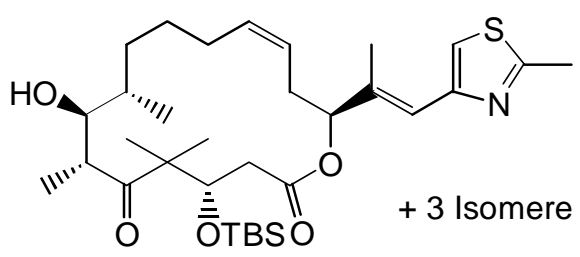

40

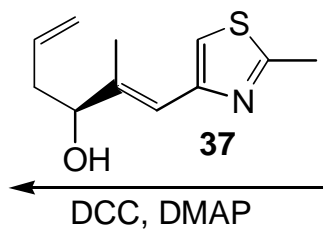<smiles>CCCCOCCCC/C=C\CCC[C@@H](C)[C@H](O)C(C)C(=O)C(C)(C)[C@H](CC(=O)O)O[Sb](=O)(=O)O</smiles>

36

Abbildung 12: Epothilone nach K. C. Nicolaou.

Diese „traceless“ Linker-Strategie nutzte K. C. Nicolaou auch bei der Synthese von (S)Zearalenon, ${ }^{[34]}$ indem er zur cyclisierenden Abspaltung eine Stille-Kupplung benutzte. 


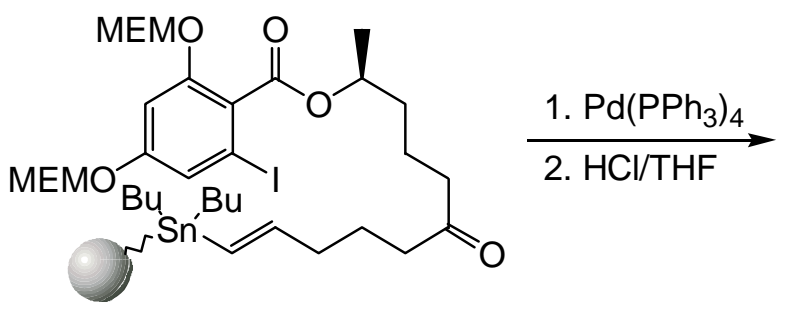

42<smiles>C[C@H]1CCCCC(=O)CCC/C=C/c2cc(O)cc(O)c2C1=O</smiles>

43

Abbildung 13: (S)-Zearalenon nach $K$. C. Nicolaou.

Eine elegante und flexible Festphasensynthese wurde von L. F. Tietze $e^{[35]}$ entwickelt, die ebenfalls eine cyclisierende Abspaltung im letzten Syntheseschritt verwendet (siehe Kapitel 8). 


\section{Palladium-katalysierte Reaktionen}

Übergangsmetall-katalysierte Reaktionen haben bereits vor vielen Jahren Einzug in die organische Synthese gehalten, wobei besonders das Palladium vielfältig eingesetzt werden kann. Neben der Wacker-Oxidation ${ }^{[36]}$ und der katalytischen Hydrierung ${ }^{[37]}$ sind leistungsfähige Methoden zur C-C- und C-X-Bindungsknüpfung entwickelt worden, die Palladium-katalysierte Reaktionen zu einem überaus wichtigen Werkzeug des synthetischen Chemikers gemacht haben. ${ }^{[38]}$

\subsection{Palladium-katalysierte allylische Alkylierung}

Palladium-katalysierte Reaktionen allylischer Substrate mit Nucleophilen wurden erstmals Mitte der 60er Jahre von $J$. Tsuji beschrieben ${ }^{[39]}$ und in den folgenden Jahren von ihm und besonders B. M. Trost weiterentwickelt. ${ }^{[40]}$ Sie zeichnen sich durch ihre große Chemo-, Regiound Stereoselektivität aus und eignen sich vor allem zum Aufbau stereogener Zentren, so daß ein Hauptanliegen der heutigen Forschung auf diesem Gebiet die Entwicklung chiraler Liganden zur enantioselektiven Synthese darstellt. ${ }^{[41]}$

Bei der allylischen Alkylierung werden $\pi$-Allyl-Palladium-Komplexe aus Substraten mit einer Abgangsgruppe in Allyl-Stellung generiert und mit Nucleophilen umgesetzt. Als Abgangsgruppen eignen sich beispielsweise Acetate, Carbonate, Chloride, Carbamate, Oxirane, Phenylether, Phosphonate und Sulfone, wobei die Reaktivität bei den Chloriden am größten ist gefolgt von den Carbonaten und Acetaten. Im ersten Schritt des Katalysecyclus ${ }^{[42]}$ (Abbildung 14) erfolgt eine Komplexierung des Olefins 45 mit der Palladium(0)-Spezies 44. Unter Inversion der Konfiguration findet anschließend eine oxidative Addition unter Ausbildung des $\eta^{1}-\sigma$-Komplexes 47 statt, welcher mit dem $\eta^{1}-\pi$-Allyl-Komplex 48 im Gleichgewicht steht. In Gegenwart von Phosphanliganden existiert weiterhin ein Gleichgewicht mit dem kationischen Komplex 49, der eine wesentlich höhere Reaktivität gegenüber einem nucleophilen Angriff besitzt. Letzterer Komplex ist bei bidentalen Liganden entropisch bevorzugt. 


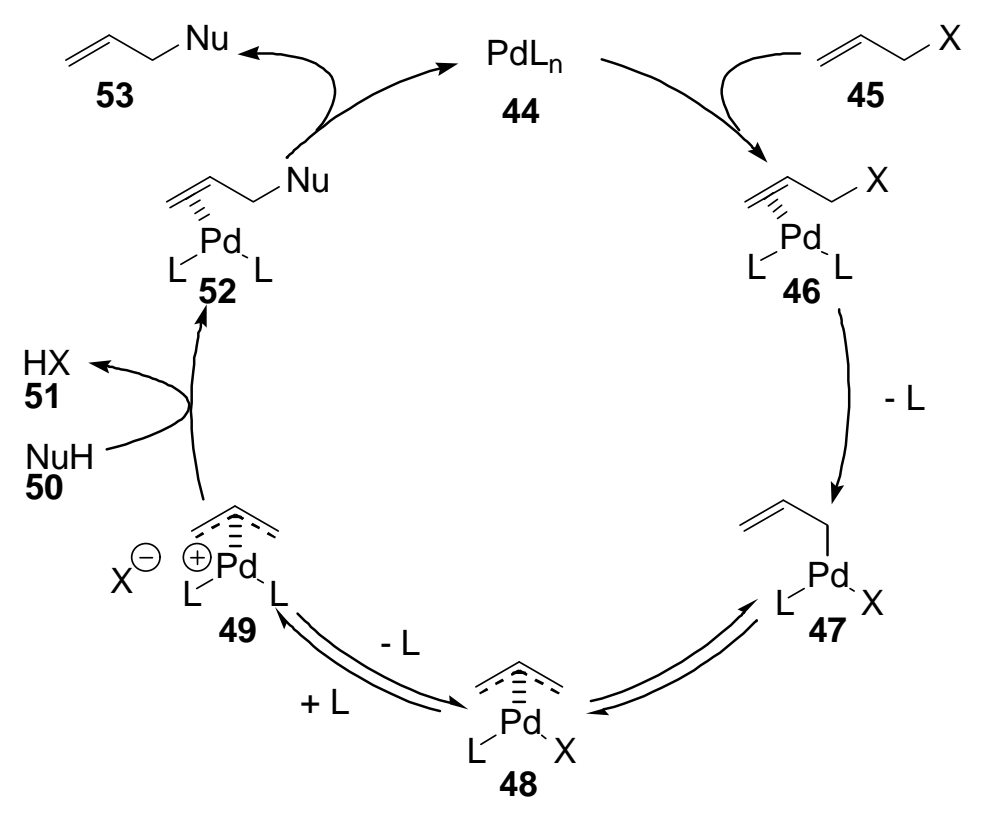

Abbildung 14: Katalysecyclus der Palladium-katalysierten allylischen Alkylierung weicher Nucleophile.

Die Nucleophile können nach ihrem Angriffsort in zwei Klassen unterteilt werden. ${ }^{[43]}$ Von weichen Nucleophilen spricht man, wenn der pKs-Wert der konjugierten Säure kleiner als 20 ist. Dies trifft auf Kohlenstoff-Nucleophile mit zwei elektronenziehenden Gruppen wie CarbonylSulfonyl-, Cyano- und Nitrogruppen zu. Auch Heteroatom-Nucleophile werden dieser Klasse zugeordnet. Verwendung finden insbesondere Stickstoff-Nucleophile wie Amine, Azide und Sulfonamide ebenso wie Sauerstoff-Nucleophile, z. B. Carboxylate und Phenole. Phosphorund Schwefel-Nucleophile können ebenfalls eingesetzt werden. ${ }^{[40 \mathrm{~d}]}$ Der Angriff des weichen Nucleophils erfolgt unabhängig von der vorherigen Position der Abgangsgruppe an einem Ende der Allyleinheit des $\pi$-Allyl-Palladium-Komplexes. Die Regioselektivität des Angriffs wird hauptsächlich durch sterische Faktoren bestimmt und erfolgt bei weichen Nucleophilen an der niedriger substituierten Seite. Durch Dissoziation des entstandenen Komplexes 52 wird der Katalysator zurückgebildet und das Produkt 53 freigesetzt.

Harte Nucleophile wie z. B. Metallorganyle, deren konjugierte Säure einen pKs-Wert größer als 20 besitzt, greifen nach dem HSAB-Prinzip ${ }^{[4]}$ das Palladium-Atom direkt unter Ausbildung eines $\eta^{3}$-Allyl- $\eta^{1}$-Alkyl-Palladium-Komplexes an. Durch reduktive Eliminierung wird unter Rückbildung des Katalysator das alkylierte Produkt erhalten.

Den stereochemischen Verlauf der allylischen Alkylierung veranschaulicht Abbildung 45. Weiche Nucleophile greifen den unter Inversion der Konfiguration gebildeten $\pi$-Allyl-PalladiumKomplex 55 von der dem Palladium gegenüberliegenden Seite aus an, so daß man als Ergebnis zweier Inversionen die Retention der Stereochemie im Produkt beobachtet. Harte Nucleophile 
hingegen werden nach Angriff auf das Metallatom durch eine reduktive Eliminierung unter Retention auf die Allyl-Einheit übertragen. Man erhält somit eine Inversion der Stereochemie.

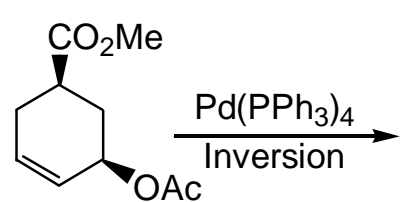

54<smiles>CCOC1=CC[C@@H](C(C)=O)CC1</smiles>

55<smiles>COC(C)C(C)C(C)=O</smiles>

58<smiles>COC(=O)[C]1CC=C[C@@H](O)C1</smiles>

59

Abbildung 15: Stereochemie der Umsetzung mit weichen bzw. harten Nucleophilen.

Die Konfiguration der Doppelbindung ist von untergeordnetem Einfluß auf die Produktbildung. Sowohl $(E)$ - als auch $(Z)$-Allylsubstrate können eingesetzt werden. In beiden Fällen erhält man $(E)$-konfigurierte Produkte. Dies läßt sich mit dem sogenannten $\pi$ - $\sigma-\pi$-Mechanis mus $^{[40 b]}$ erklären (siehe Abbildung 16). Der sich zunächst bildende syn-anti-Allyl-Komplex ${ }^{[45]}$ 61 bzw. 62 wandelt sich in den stabileren syn-syn-Komplex 68 um, der schließlich nucleophil angegriffen wird. Dieser Vorgang verläuft schnell im Vergleich mit den übrigen Schritten des Katalysecyclus, so daß selektiv Verbindungen mit $(E)$-konfigurierter Doppelbindung erhalten werden. 


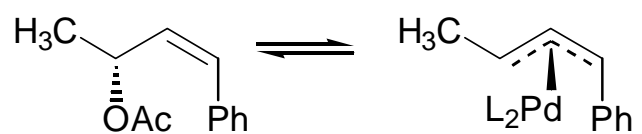

60<smiles>C=CCCCCC</smiles>

62<smiles>C/C=C\[C@H](OC(C)=O)c1ccccc1</smiles>

63<smiles>[CH][Pb](/C=C/C)(c1ccccc1)[C@H](/C=C/C)c1ccccc1</smiles>

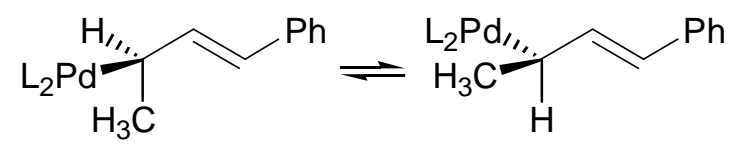

64

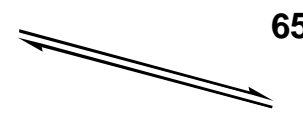

65<smiles>C=CC=C</smiles><smiles>C=[Tl]</smiles>

66

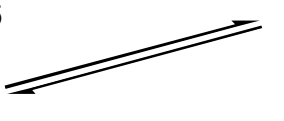

67<smiles>CCCC=Cc1ccccc1</smiles>

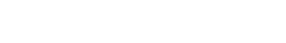

68<smiles>COC(C)C(C)/C=C/c1ccccc1</smiles>
$\mathrm{NaCH}\left(\mathrm{CO}_{2} \mathrm{Me}\right)_{2}$<smiles>C/C=C/[C@H](C(C)=O)c1ccccc1</smiles>

69 $90: 10$ 70

Abbildung 16: $\pi-\sigma-\pi-$ Mechanismus.

Bei den in der Literatur beschriebenen allylischen Alkylierungen werden häufig deprotonierte 1,3-Dicarbonylverbindungen mit dem aus Allylacetaten erzeugten $\pi$-Allyl-Palladium-Komplex umgesetzt. Eine interessante Variante ist die Verwendung von Allylcarbonaten 71, ${ }^{[46]}$ da hier bei der Bildung des Palladium-Komplexes 72 die Carbonat-Gruppe abgespalten wird, welche spontan decarboxyliert unter Ausbildung eines Alkoxid-Restes 74. Dieser wiederum kann nun das Nucleophil deprotonieren. Es muß also keine Base extra zugesetzt werden, was die Ökonomie der Umsetzung erhöht.

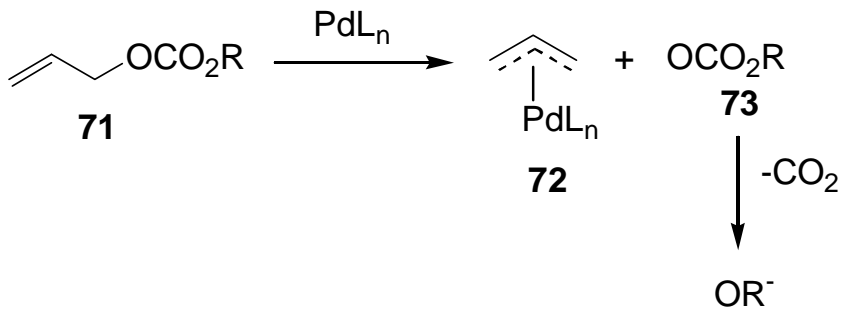

74

Abbildung 17: Allylische Alkylierung mit Allylcarbonaten. 


\subsection{Die Heck-Reaktion}

Im Jahre 1968 beschrieb $R$. F. Heck die nach ihm benannte Palladium-katalysierte Kupplung von Aryl- und Alkenylhalogeniden mit Alkenen. ${ }^{[47]}$ Nachdem diese Methode für viele Jahre ein Schattendasein fristete, begann vor knapp 15 Jahren ihr steiler Aufstieg zu einer der vielseitigsten und nützlichsten Transformationen in der organischen Synthese. Sie inspirierte die Entwicklung verwandter Palladium-katalysierter Umsetzungen, die mit Namen wie Stille, Suzuki und Sonogashira verbunden sind. ${ }^{[48]}$ Die große Beliebtheit der Heck-Reaktion beruht auf der Kompatibilität mit zahlreichen funktionellen Gruppen, was den Einsatz zum Aufbau hochkomplexer Strukturen erlaubt, den milden und sehr variationsfähigen Reaktionsbedingungen und der geringen Menge an benötigtem Katalysator. Nicht zu vergessen ist die Möglichkeit der enantioselektiven Reaktionsführung, wodurch diese Methode zusätzlich an Interesse gewinnt.

Der exakte Reaktionsverlauf ist bis heute nicht in allen Details geklärt. Der im folgenden beschriebene Mechanismus stellt jedoch eine allgemein akzeptierte Arbeitshypothese dar, die zur Vorhersage und Deutung der Versuchsergebnisse gute Dienste leistet. ${ }^{[48 \mathrm{~b}]}$

Als katalytisch aktive Spezies wird der koordinativ ungesättigte 14-Elektronen-Palladium(0)Komplex 77 angenommen (Abbildung 18). Er wird aus Palladium(II)-Salzen wie z. B. Palladiumacetat durch Reduktion gebildet. Als Reduktionsmittel wirken als Base zugesetzte tertiäre Amine (Abbildung 19), ${ }^{[4]}$ Phosphane oder das Olefin selbst. Eine andere Möglichkeit ist die Verwendung von Palladium(0)-Verbindungen wie z. B. Tetrakis(triphenylphosphan)palladium 75, das in Lösung als Tris(triphenylphosphan)palladium $\mathbf{7 6}$ vorliegt und durch endergonischen Verlust eines weiteren Phosphanliganden in die aktive Spezies 77 übergeht. 


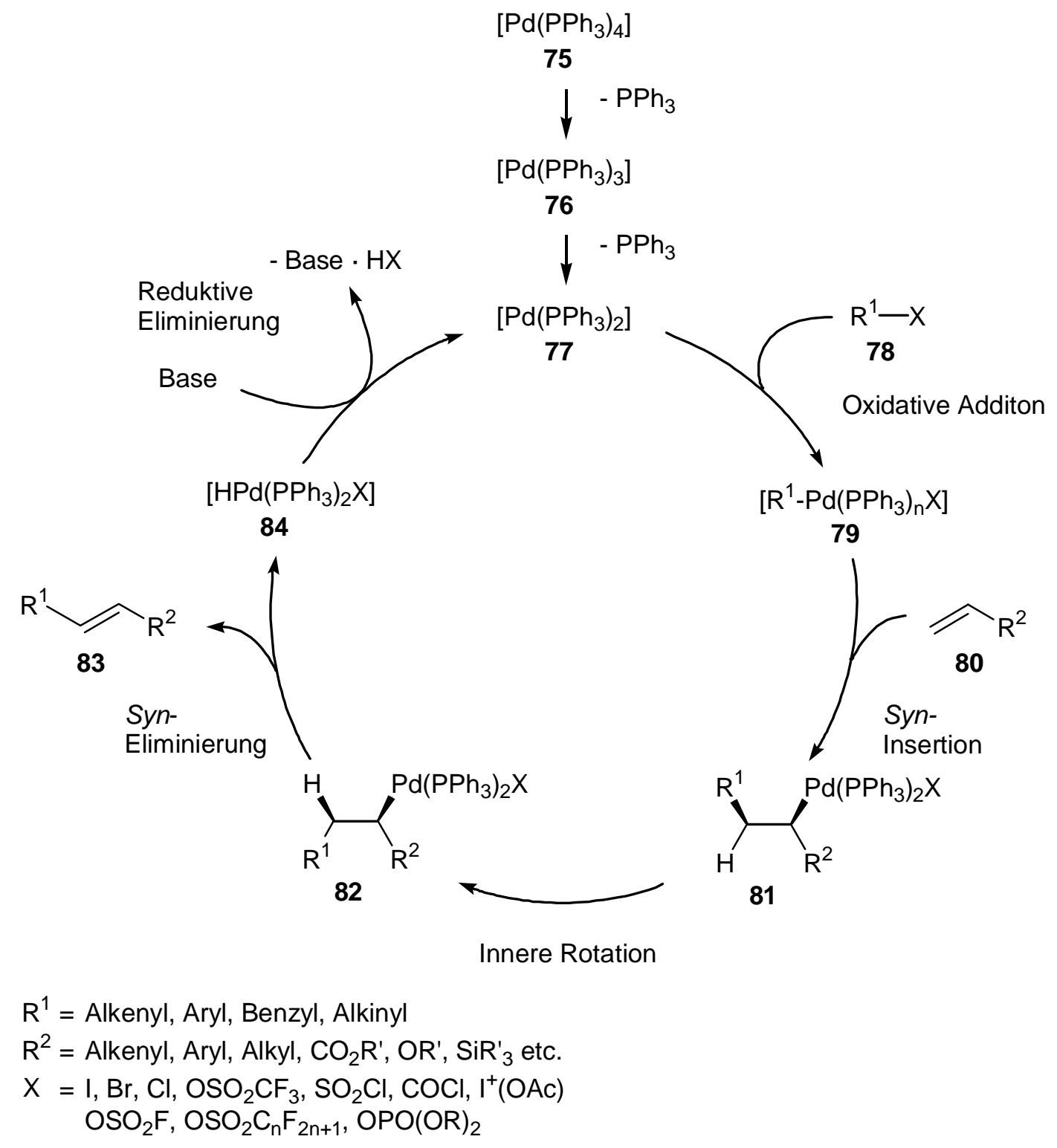

Abbildung 18: Katalysecyclus der Heck-Reaktion.

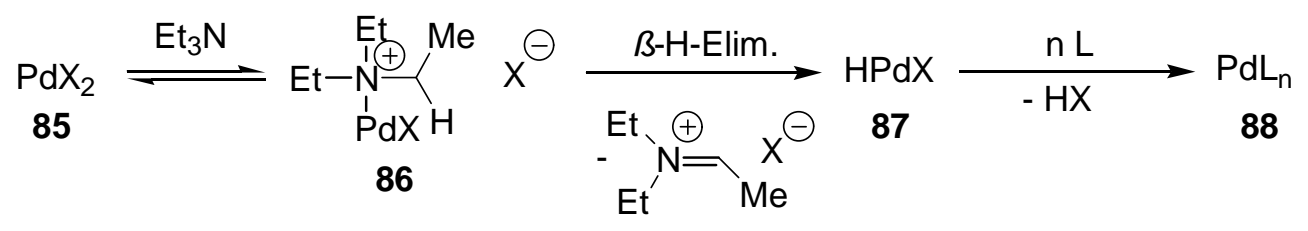

Abbildung 19: Reduktion von Pd(II)-Salzen durch Amine.

Weitere häufig verwendete Palladium(0)-Katalysatoren sind die Dibenzylidenaceton-Komplexe $\mathrm{Pd}(\mathrm{dba})_{2}$ und $\mathrm{Pd}_{2}(\mathrm{dba})_{3} \cdot \mathrm{CHCl}_{3}$ sowie der von W. A. Herrmann und M. Beller ${ }^{[50]}$ entwickelte Phosphapalladacyclus 89 (Abbildung 20). Dieser hat den Vorteil, daß er auch oberhalb von 
$120{ }^{\circ} \mathrm{C}$ stabil ist, während sich die üblicherweise als Liganden eingesetzten Phosphane bei dieser Temperatur durch Spaltung der Kohlenstoff-Phosphor-Bindung zersetzen.

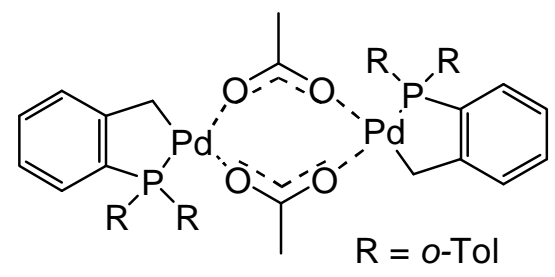

89

Abbildung 20: Herrmann-Beller-Katalysator.

Im ersten Schritt des Katalysecyclus erfolgt die oxidative Addition des Aryl- oder AlkenylHalogenids 78 an die aktive Spezies 77 unter Ausbildung eines $\sigma$-Aryl- oder $\sigma$-AlkenylPalladium(II)-Komplexes 79. Elektronenarme Substrate sind bei der oxidativen Addition reaktiver als elektronenreiche, und die Reaktivität nimmt mit abnehmender Bindungsstärke der $\mathrm{C}\left(\mathrm{sp}^{2}\right)$-X-Bindung in der Reihe $\mathrm{Cl} \ll \mathrm{Br}<\mathrm{OTf}<\mathrm{I}$ zu. Elektronenreiche Phosphanliganden beschleunigen die oxidative Addition. Als zweiter Schritt schließt sich die Insertion des Olefins 80 an. Nach Koordination des Alkens an den Palladium-Komplex, bei der ein anderer Ligand abgespalten wird, erfolgt die Insertion als eine Wanderung eines $\sigma$-Liganden vom Palladium auf den ungesättigten Liganden. Voraussetzung für diesen Vorgang ist eine cis-Orientierung des Alkens und des $\sigma$-Liganden, was durch Rotation aus der ,out-of-plane“-Konformation in die ,in-plane“-Konformation ${ }^{[48 b]}$ erreicht wird. Über einen Vier-Zentren-Übergangszustand kommt es in Form einer cis-selektiven Carbopalladierung zur Bindungsbildung zwischen Metall und Olefin. Dieser Koordinatons-Insertions-Prozeß kann in Abhängigkeit von der Natur des austretenden Liganden und von Additiven einen neutralen oder kationischen Weg verfolgen (Abbildung 21). ${ }^{[48 f]}$ Bei Verwendung monodentaler Liganden wird gewöhnlich der neutrale Pfad durchlaufen, bidentale Liganden hingegen favorisieren den kationischen Weg. Letzterer kann auch durch Benutzung von Aryl- oder Alkenyl-Triflaten aufgrund der schwachen Palladium-Triflat-Bindung $^{[51]}$ sowie durch Zusatz von Silber- oder Thalliumsalzen bei Umsetzungen mit Aryl- oder Alkenylhalogeniden infolge der Bildung schwerlöslicher Salze gezielt eingeschlagen werden. 


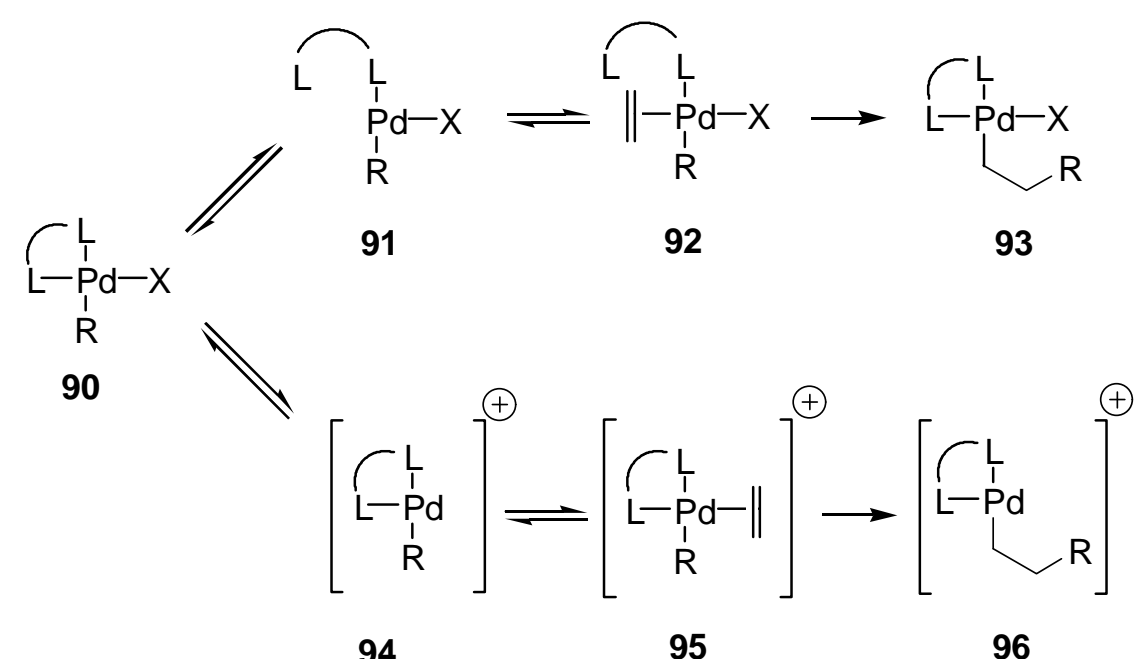

Abbildung 21: Neutraler und kationischer Koordinations-Insertions-Mechanismus.

Die Regiochemie der Olefininsertion wird durch sterische und elektronische Effekte kontrolliert, wobei erstere bei elektronenarmen oder nichtaktivierten Alkenen überwiegen, so daß die Einfügung des organischen Restes an der geringer substituierten Position erfolgt. Bei Verwendung elektronenreicher Olefine ist eine Vorhersage der Regiochemie häufig schwierig, da sich hier elektronische und sterische Effekte oft gegensinnig auswirken.

Zur Abspaltung des Produktes $\mathbf{8 3}$ durch eine $\beta$-Eliminierung ist eine synperiplanare Anordung des Palladiums bezüglich der Bindung zu einem $\beta$-ständigen Wasserstoffatom nötig. Hierzu erfolgt eine innere Rotation von 81, um die syn-Eliminierung zu ermöglichen. Der Eliminierungsschritt ist reversibel, so daß eine Readdition der entstandenen Hydrido-Palladium-Spezies 84 an 83 auftreten kann, was zu einer Isomerisierung der Doppelbindung führt. Wird ein terminales Alken eingesetzt, wird generell das thermodynamisch stabilere (E)-Alken $\mathbf{8 3}$ erhalten. Im letzten Schritt des Katalysecyclus regeneriert die zugesetzte Base den aktiven Palladium(0)Katalysator durch reduktive Eliminierung von HX aus 84, wodurch der Cyclus geschlossen wird.

\subsection{Ausgewählte Beispiele Palladium-katalysierter Reaktionen}

Beim Aufbau hochkomplexer Strukturen wie Naturstoffen können Synthesemethoden ihre Leistungsfähigkeit unter Beweis stellen. Dies gelang der Heck-Reaktion z. B. als Schlüsselschritt in der Morphin-Synthese von L. E. Overman, ${ }^{[52]}$ bei der ein quartäres Kohlenstoffzentrum aufgebaut wird. 
<smiles>C=C1C=C2CCN(C(C)=O)[C@H](Cc3ccc(OC)c(O)c3I)[C@H]2CC1</smiles>

97
$20 \%$ Kat. PMP, Toluol, $120^{\circ} \mathrm{C}$

$56 \%$

98

$$
\text { Kat. }=\left[\mathrm{Pd}\left(\mathrm{OC}(\mathrm{O}) \mathrm{CF}_{3}\right)_{2}\left(\mathrm{PPh}_{3}\right)_{2}\right]
$$
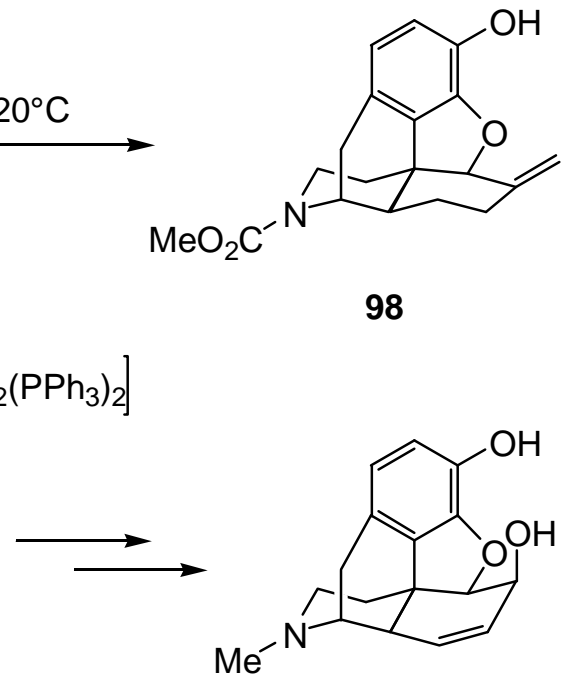

99

(-)-Morphin

Abbildung 22: (-)-Morphin-Synthese von L. E. Overman.

Auch enantioselektive Heck-Reaktionen konnten in der Naturstoffsynthese eingesetzt werden. In seinem Zugang zu dem indirekten Parasympathomimetikum (-)-Physostigmin 102 gelang L. E. Overman die Cyclisierung von 100 mit einem Enantiomerenüberschuß von 95\%. ${ }^{[53]}$<smiles>COc1ccc(N(C)C(=O)/C(C)=C\CO[SnH]2CCCCC2)c(I)c1</smiles>

100
1) $\mathrm{Pd}_{2} \mathrm{dba}_{3} \cdot \mathrm{CHCl}_{3}$ (S)-BINAP, PMP 2) $\mathrm{HCl}$ $84 \%, 95 \%$ ee<smiles>CNC(=O)Oc1ccc2c(c1)[C@]1(C)CCN(C)[C@@H]1N2C</smiles>
102

(-)-Physostigmin

Abbildung 23: (-)-Physostigmin-Synthese von L. E. Overman.

Ein elegantes Beispiel zur Synthese von Steroiden und Steroid-Analoga wurde von L. F. Tietze $e^{[54]}$ publiziert. Unter Ausnutzung der höheren Reaktivität von Vinylbromiden gegenüber Arylbromiden konnte das (Z)-Vinylbromid 103 mit einer Diastereoselektivität von über 98\% mit dem CD-Baustein 104 umgesetzt werden. Die anschließende Cyclisierung gelang mit dem Palladacyclus 89 in nahezu quantitativer Ausbeute. 


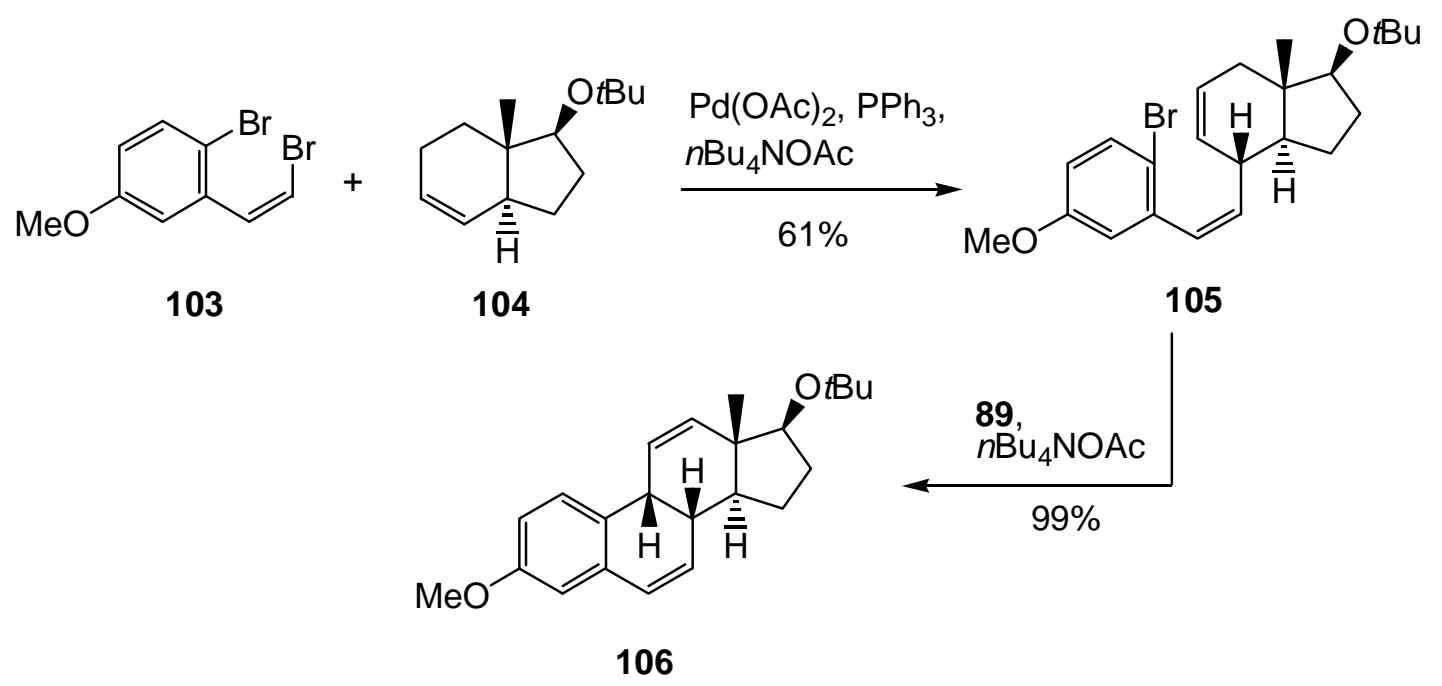

Abbildung 24: Steroid-Synthese von L. F. Tietze.

Durch die verschiedenen Arten von einsetzbaren Nucleophilen hat sich die allylische Alkylierung zu einem wichtigen Synthesewerkzeug entwickelt. Besonders elegant können mit dieser Methode mittlere und große Ringe aufgebaut werden, wie das Beispiel des 26-gliedrigen Makrocyclus 108 zeigt. $^{[55]}$

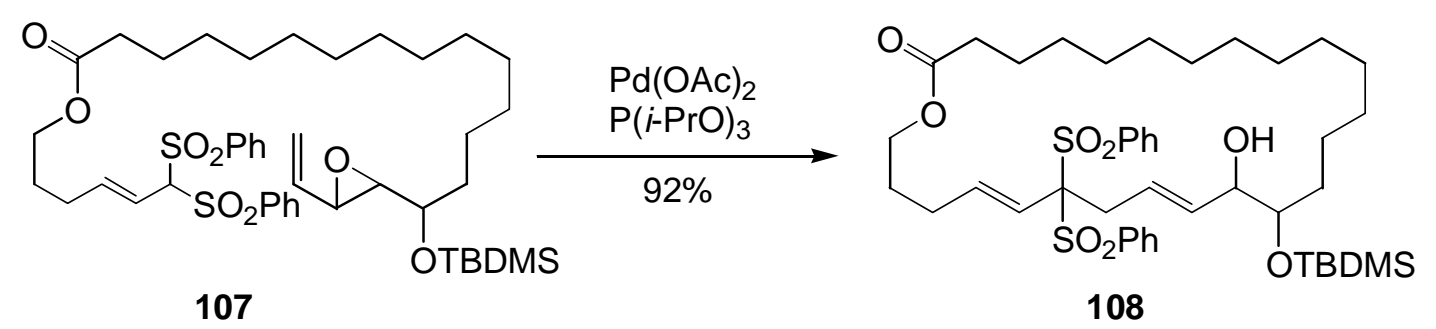

Abbildung 25: Aufbau von Makrocyclen durch allylische Alkylierung.

Mit guter Enantioselektivität von 94\% konnte B. M. Trost ${ }^{[56]}$ den prochiralen Diester 109 mit 6-Chlorpurin 110 in Gegenwart des $C_{2}$-symmetrischen Diphosphans 111 umsetzten. Nach weiteren Transformationen wurde das Carbanucleosid Neplanocin 113 erhalten. 


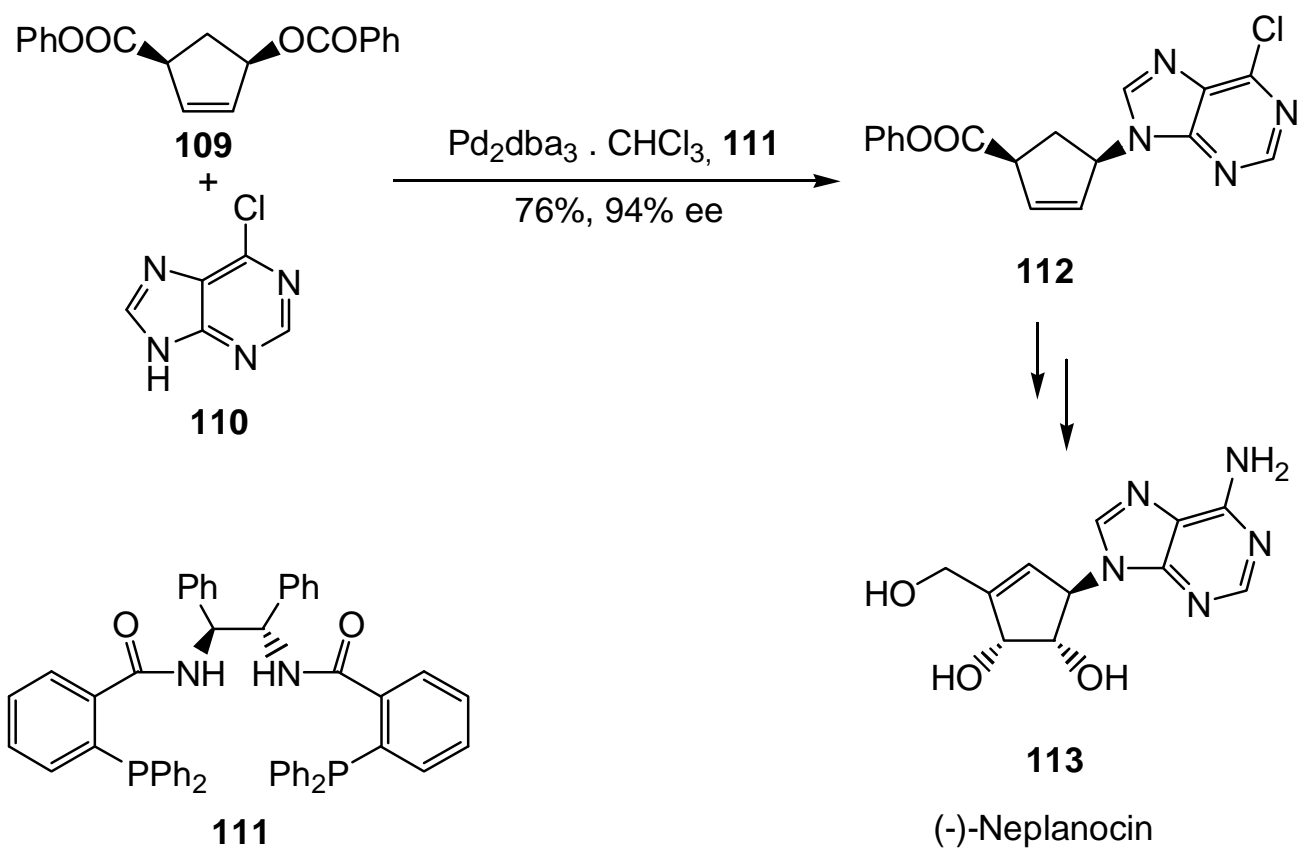

Abbildung 26: (-)-Neplanocin-Synthese von B. M. Trost.

Die Vielseitigkeit Palladium-katalysierter Reaktionen zeigt die sehr kurze Synthese von Cephalotaxin 117. Durch Kombination einer allylischen Aminierung zum Aufbau der SpiroVerbindung 115 und einer intramolekularen Heck-Reaktion zur Generierung des 7-Ringes gelang L. F. Tietze $e^{[57]}$ die Synthese der Verbindung 116, die in vier Stufen in Cephalotaxin 117 überführt werden kann.
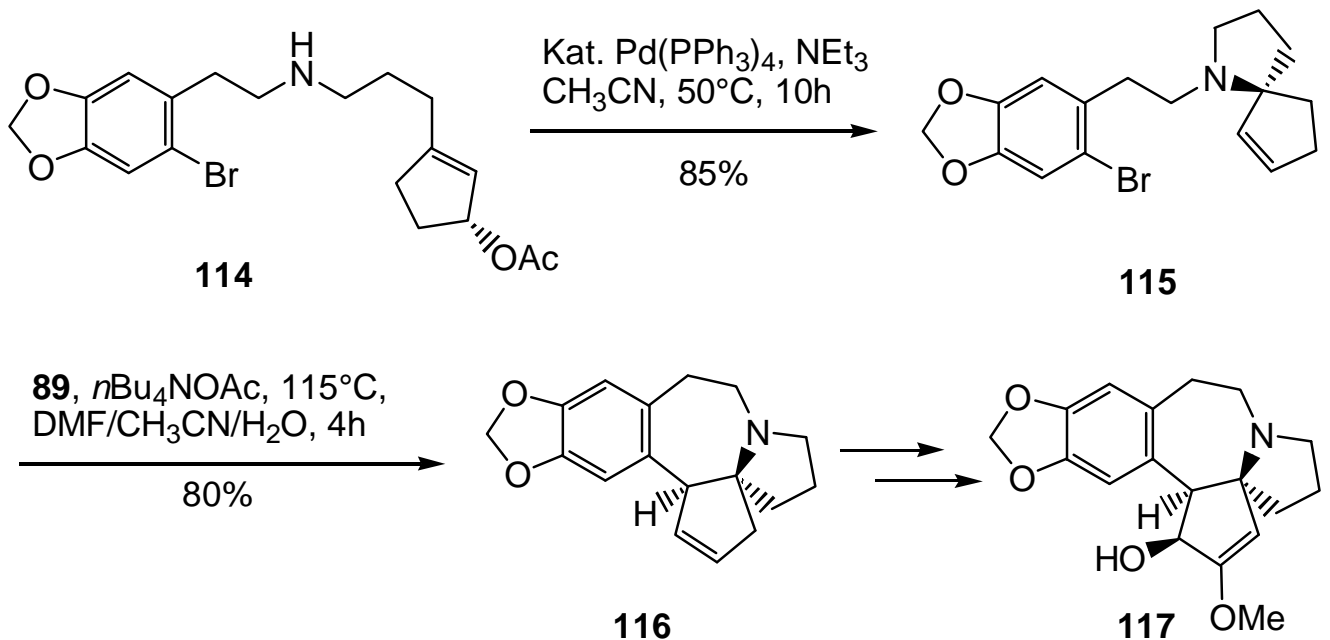

Cephalotaxin

Abbildung 27: Cephalotaxin-Synthese von L. F. Tietze. 


\section{Aufgabenstellung und Planung der Arbeit}

Die Festphasensynthese kleiner, organischer Moleküle gewinnt dank ihrer Anwendung im Rahmen der Kombinatorischen Chemie und deren Nutzung für die Suche nach neuen Wirkstoffen ein immer größeres Interesse. Sie bietet einen schnellen und effizienten Zugang zu groBen Substanzbibliotheken, die mit Hilfe des High-Throughput-Screenings innerhalb kürzester Zeit auf ihre biologische Aktivität hin getestet werden können. Besonders die leichte Reinigung und Isolierung polymergebundener Produkte durch einfache Waschprozeduren erlaubt eine Automatisierung der Synthese an der festen Phase und somit eine deutliche Beschleunigung des Aufbaus von Substanzbibliotheken.

Zur Durchführung effektiver Synthesen am polymeren Träger ist es unerläßlich, ein großes Repertoire an Synthesemethoden zur Verfügung zu haben. Ein wichtiges Gebiet stellen dabei übergangsmetallkatalysierte C-C-Verknüpfungsreaktionen dar. Hierbei sei neben der HeckReaktion besonders die Palladium-katalysierte allylische Alkylierung erwähnt. Mit ihr lassen sich Nucleophile, bevorzugt weiche, stabilisierte Carbanionen wie z. B. 1,3-Dicarbonylverbindungen, durch Substrate mit einer allylischen Abgangsgruppe in guten Ausbeuten und hoher Stereokontrolle alkylieren. Durch die große Variabilität der Allyl-Komponente und der hohen Toleranz funktioneller Gruppen ist diese Reaktion von großem Interesse für die Kombinatorische Chemie.

Im Rahmen der vorliegenden Arbeit sollte die Palladium-katalysierte allylische Alkylierung von festphasengebundenen 1,3-Dicarbonylverbindungen 118 etabliert werden. Neben der Optimierung der Reaktion ist es für die breite Anwendbarkeit der Reaktion erforderlich, unterschiedliche Abspaltungsmethoden zu entwickeln sowie verschiedene Trägerharze zu verwenden. Um eine hohe Diversität der Produkte zu gewährleisten, sollten sowohl verschiedene 1,3Dicarbonylverbindungen 118 als auch unterschiedliche Allylkomponenten 119, auch Bisallyltemplate, zum Einsatz kommen. Die Möglichkeit von Folgereaktionen am Harz erhöhen zusätzlich die Flexibilität der Methode. Hierzu sollten Heck-Reaktionen mit den polymergebundenen Olefinen 120 und 121 durchgeführt werden. 


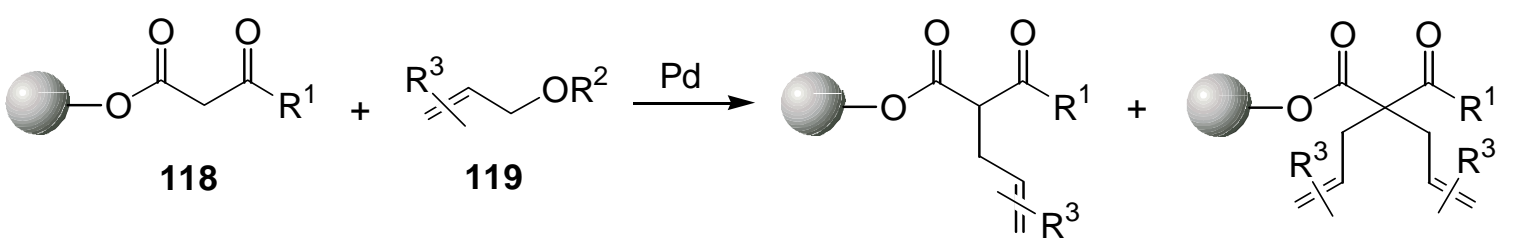

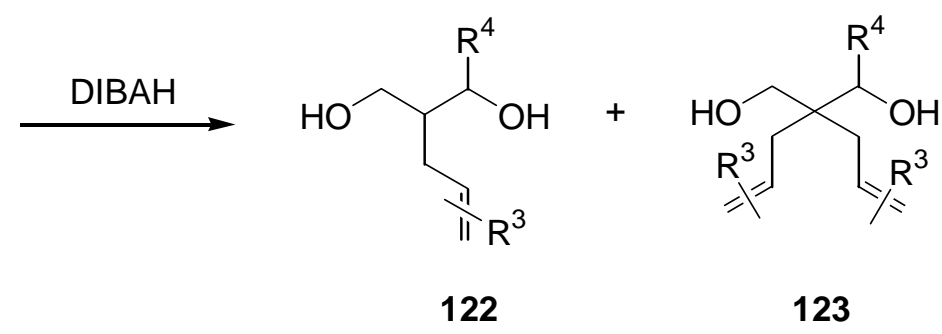

Ein weiteres Ziel bestand in der Festphasensynthese von Pyrazolonen unter Verwendung unterschiedlich substituierter Hydrazine 125. Dabei sollte auf eine von L. F. Tietze und A. Steinmetz ${ }^{[14 \mathrm{a}, 35]}$ entwickelte Reaktionssequenz zurückgegriffen werden. Durch die Variation der Hydrazin-Komponente sollte die Diversität der synthetisierten Heterocyclen deutlich erhöht werden.

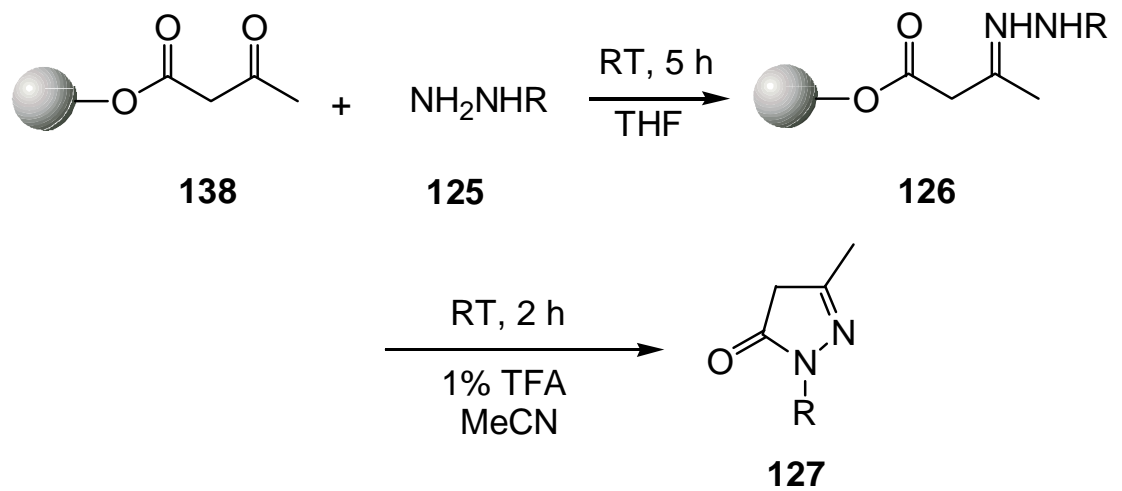

Zusammenfassend waren folgende Aufgaben zu bearbeiten:

1. Synthese der polymergebundenen 1,3-Dicarbonylverbindungen.

2. Synthese unterschiedlich substituierter Allyl-Komponenten mit verschiedenen allylischen Abgangsgruppen.

3. Palladium-katalysierte allylische Alkylierung an unterschiedlichen polymeren Trägern.

4. Untersuchung verschiedener Abspaltungsreaktionen.

5. Verwendung von Bisallyltemplaten in der Palladium-katalysierte allylische Alkylierung.

6. Heck-Reaktionen mit polymergebundenen Olefinen.

7. Festphasensynthese von Pyrazolonen. 


\section{Synthese der Edukte}

\subsection{Synthese von spacermodifiziertem Merrifield-Harz 6}

In Anlehnung an eine von L. F. Tietze und A. Steinmetz entwickelte Methode ${ }^{[14 a]}$ wurde Merrifield-Harz 1 (2\% DVB, 2-2.5 mmol Cl/g) mit einem Spacer versehen, indem es mit 5 Äquivalenten Mononatrium-1,3-propandiolat 128 und 0.1 Äquivalenten $n$-Tetrabutylammoniumiodid versetzt und für 24 Stunden bei Raumtemperatur unter Lichtausschluß gerührt wurde. Die Reinigung des Harzes erfolgte nach Zugabe von Wasser durch gründliches Waschen mit Dimethylformamid, Methanol und Dichlormethan. Im Gegensatz zu der ursprünglichen Vorschrift konnte die Reaktionstemperatur durch Zugabe des Iodids von $85{ }^{\circ} \mathrm{C}$ auf Raumtemperatur gesenkt werden. Ferner ist die „optische Qualität“ des spacermodifizierten Harzes deutlich besser, da es nicht mehr zu einer bräunlichen Verfärbung kommt, sondern das Harz seine gelblich-weiße Farbe behält.

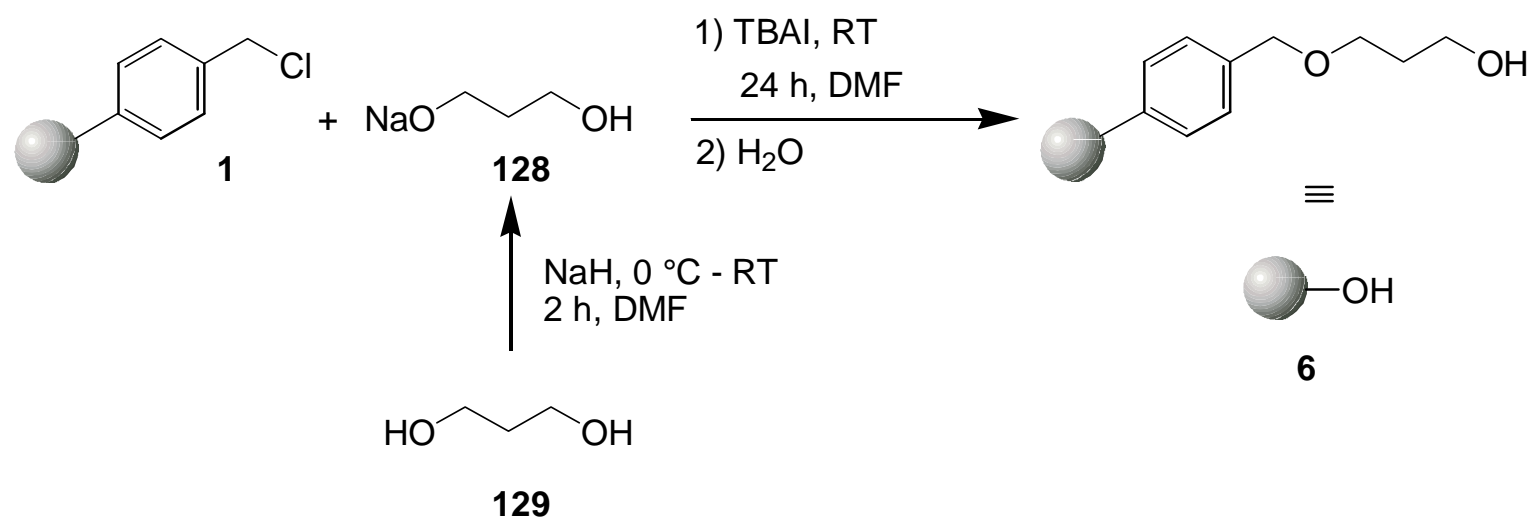

Zum Nachweis der Vollständigkeit der Umsetzung führte man eine Beilstein-Probe ${ }^{[58]}$ durch, die durch Ausbleiben einer grünen Flammenfärbung die Abwesenheit von Chlor zeigte. Um die Konzentration der freien Hydroxy-Gruppen $\mathrm{zu}$ bestimmen, wurde das Harz 6 mit 10 Äquivalenten 3,5-Dinitrobenzoylchlorid 130 umgesetzt. Anschließende elementaranalytische Bestimmung des Stickstoffgehalts ergab eine Beladungsdichte von 0.50 - 0.75 mmol Hydroxy-Gruppen pro Gramm spacermodifiziertes Harz 6. Auf diese Konzentration wurden im folgenden die Ausbeuten der Reaktionen an der festen Phase bezogen. 


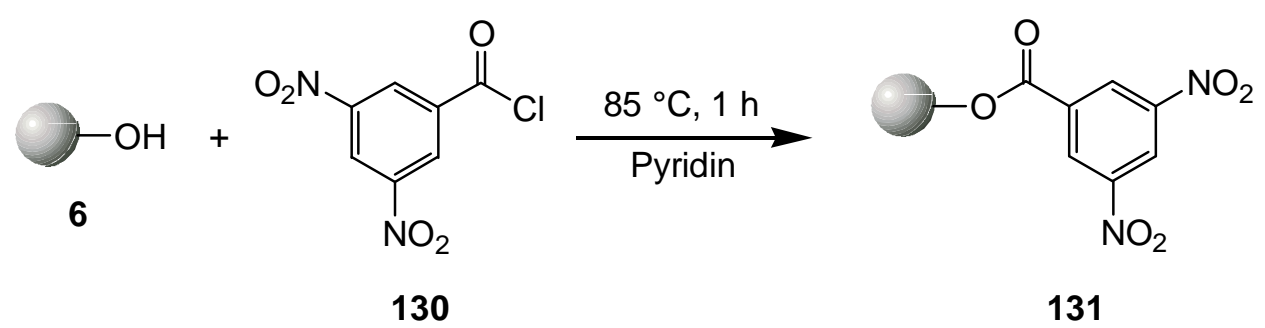

Eine weitere Methode zur Ermittelung der Beladungsdichte besteht in der Synthese von festphasengebundener Benzoesäure 133 durch Veresterung des Spacer-Harzes 6 mit 5 Äquivalenten Benzoylchlorid in Gegenwart von Triethylamin und 4-Dimethylaminopyridin. Durch reduktive Abspaltung von $\mathbf{1 3 3}$ konnten die Ergebnisse des elemantaranalytischen Verfahrens durch eine praxisnähere Reaktionssequenz bestätigt werden.

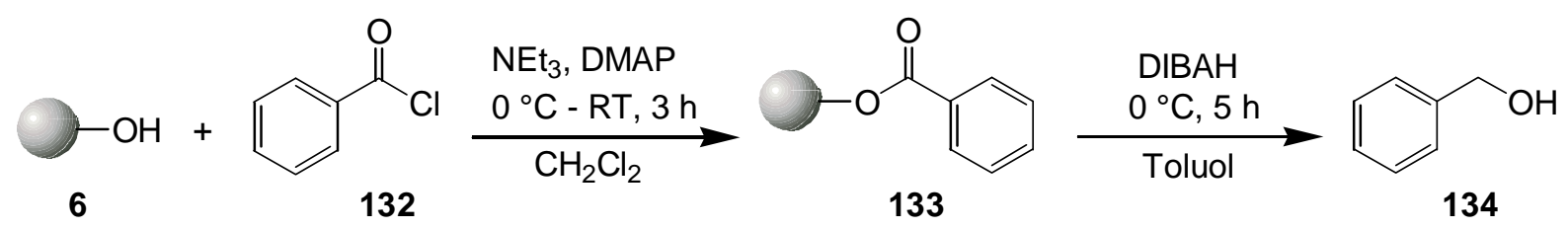

Die unterschiedlichen Beladungsdichten sind abhängig von der jeweils verwendeten MerrifieldHarz-Charge, innerhalb einer Charge wurden reproduzierbare Beladungen erzielt. Dies zeigt, daß die Qualität der käuflichen Harze deutlichen Schwankungen unterworfen ist. Abgesehen von variierenden Konzentrationen freier Hydroxy-Gruppen konnten jedoch keine Reaktivitätsunterschiede bezüglich der durchgeführten Festphasensynthesen festgestellt werden.

Ein Vergleich der Konzentration der reaktiven Zentren im Merrifield-Harz 1 ( 2.0 - $2.5 \mathrm{mmol} / \mathrm{g}$ ) und im spacermodifizierten Harz 6 ( $0.50-0.75 \mathrm{mmol} / \mathrm{g})$ zeigt, daß ein gewisser Anteil von querverknüpfenden Reaktionen stattgefunden hat, bei denen beide Seiten des Propanolats 128 mit dem polymeren Träger reagiert haben. Dies ist verständlich aufgrund der vergleichsweise hohen Beladungsdichte von ca. $2.5 \mathrm{mmol} \mathrm{Cl} / \mathrm{g}$ des Harzes 1 und der damit einhergehenden geringen räumlichen Trennung der reaktiven Zentren an der festen Phase. Die zusätzlichen Querverknüpfungen haben aber keinen negativen Einfluß auf die Quelleigenschaften des Harzes 6.

\section{Diskussion der spektroskopischen Daten}

Das IR-Spektrum von 131 weist drei neue Absorptionsbanden gegenüber dem von 6 auf. Es handelt sich dabei um das Signal der Esterfunktion bei $1732 \mathrm{~cm}^{-1}$ und um Banden bei 1510 und $1340 \mathrm{~cm}^{-1}$, die den Streckschwingungen der Nitro-Gruppe entsprechen. 
Im IR-Spektrum der festphasengebundenen Benzoesäure 133 erkennt man deutlich das Signal der Carbonyl-Gruppe bei $1718 \mathrm{~cm}^{-1}$.

\subsection{Synthese der polymergebundenen $1,3-$-Dicarbonylverbindungen 138 und 143}

Die Synthese von polymergebundenem Acetoacetat 138 gelang durch Transacylierung des Spacer-Harzes 6 mit 10 Äquivalenten Acetessigsäure-t-butylester 135 durch Erhitzen in Toluol. ${ }^{[59]}$ Es handelt sich dabei um ein einfaches und effizientes Verfahren, das ohne Zusatz von Katalysatoren auskommt. Als reaktive Spezies tritt das Acetylketen 136 auf.

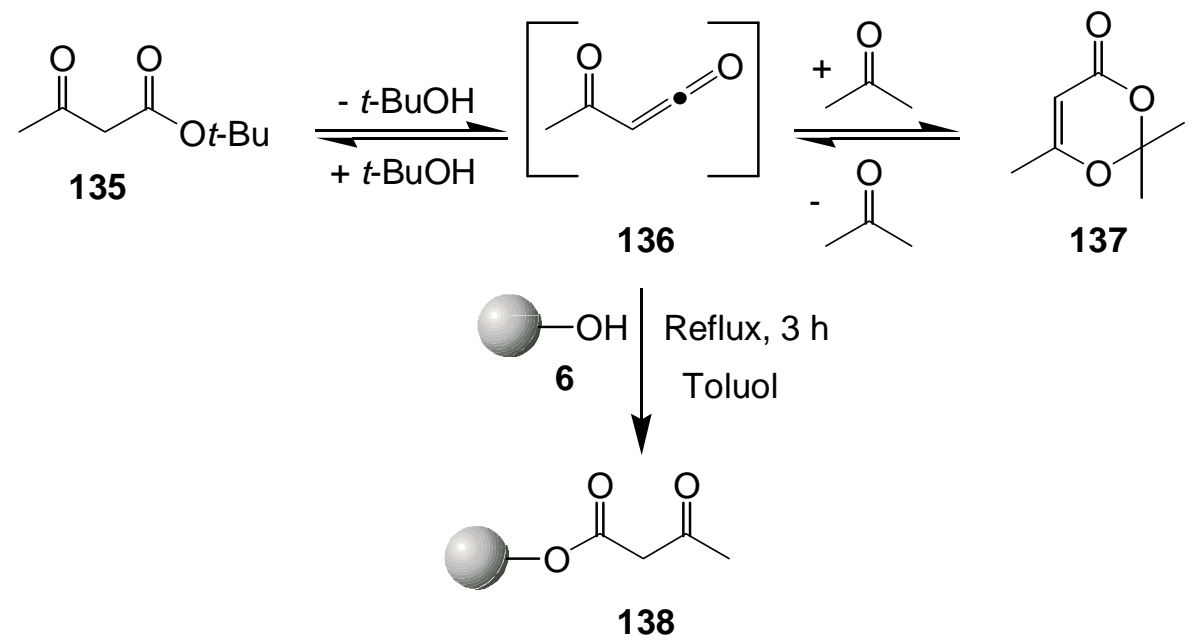

Ein anderer Zugang zu polymergebundenem Acetoacetat 138 besteht in der thermischen Umsetzung des Spacer-Harzes 6 mit 2,2,6-Trimethyl-dioxin-4-on 137, wobei auch hier 136 als reaktive Zwischenstufe auftritt. Durch seinen hohen Preis und seine geringere Reaktivität ${ }^{[60]}$ ist der Weg über 137 jedoch der erstgenannten Methode unterlegen. Eine weitere Möglichkeit besteht in der Verwendung von Diketen. Aufgrund der Toxizität und extremen Hydrolyseempfindlichkeit von Diketen ist aber das hier beschriebene Verfahren mit dem kostengünstigen und leicht handhabbaren Acetessigsäure-t-butylester zu bevorzugen.

Einen Hinweis auf die Beladung des Harzes 138 mit Acetoacetat gibt die von L. F. Tietze und A. Steinmetz durchgeführte Synthese von 3-Methyl-1-phenyl-2-pyrazol-5-on 141 durch Bildung des polymergebundenen Hydrazons 140 mit Phenylhydrazin 139 und nachfolgender, cyclisierender Abspaltung vom polymeren Träger. ${ }^{[14 a, 35]}$ Unter Annahme quantitativer Umsetzungen kann auf eine Konzentration von 0.50 - 0.75 mmol Acetoacetat pro Gramm Harz 138 geschlossen werden, was entsprechend der Beladungsdichte des jeweiligen Spacer-Harzes eine vollständigen Veresterung der freien Hydroxy-Gruppen in $\mathbf{6}$ bedeutet. 


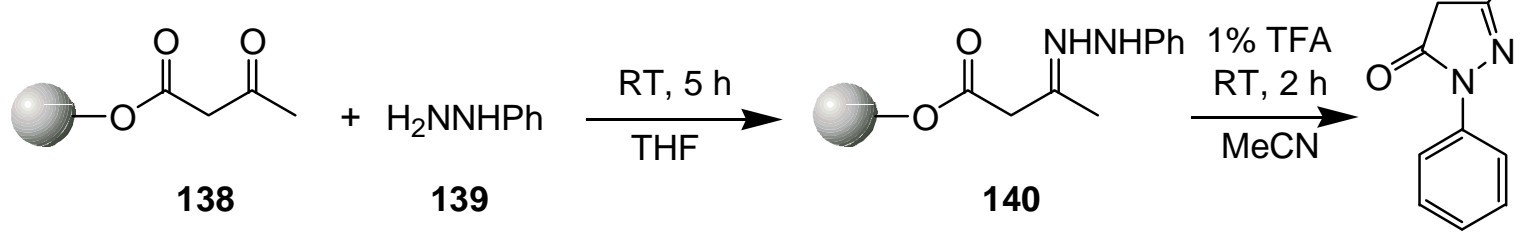

Zur Synthese von festphasengebundenem Methylmalonat 143 konnte auf eine von L. F. Tietze und A. Steinmetz ${ }^{[14 \mathrm{a}, 35]}$ etablierte Prozedur zurïckgegriffen werden, indem spacermodifiziertes Merrifield-Harz 6 mit 5 Äquivalenten Malonsäuremonomethylesterchlorid 142 unter Zusatz von Triethylamin umgesetzt wurde.<smiles>CCNC(=O)[C@H](CC)c1ccc(OC(=O)CC(=O)OC)cc1</smiles>

\section{Diskussion der spektroskopischen Daten von 138 und 143}

Die Entstehung von polymergebundenem Acetoacetat kann man deutlich im IR-Spektrum des Harzes 138 erkennen. Es entstehen zwei neue, intensive Banden bei 1742 und $1718 \mathrm{~cm}^{-1}$, die der Ester- bzw. der Ketogruppe zugeordenet werden können.

Im IR-Spektrum von 143 erkennt man eine starke Bande bei $1734 \mathrm{~cm}^{-1}$, die dem Signal der Ester-Funktionen entspricht.

\subsection{Synthese der Allylacetate}

Für die Darstellung von Allylacetaten existieren eine Reihe von synthetischen Methoden. Ein sehr einfacher und effizienter Zugang besteht in der Acetylierung von Allylalkoholen, welche sich wiederum durch Reduktion von $\alpha, \beta$-ungesättigten Carbonylverbindungen oder durch eine Grignard-Reaktion mit Aldehyden aufbauen lassen. 


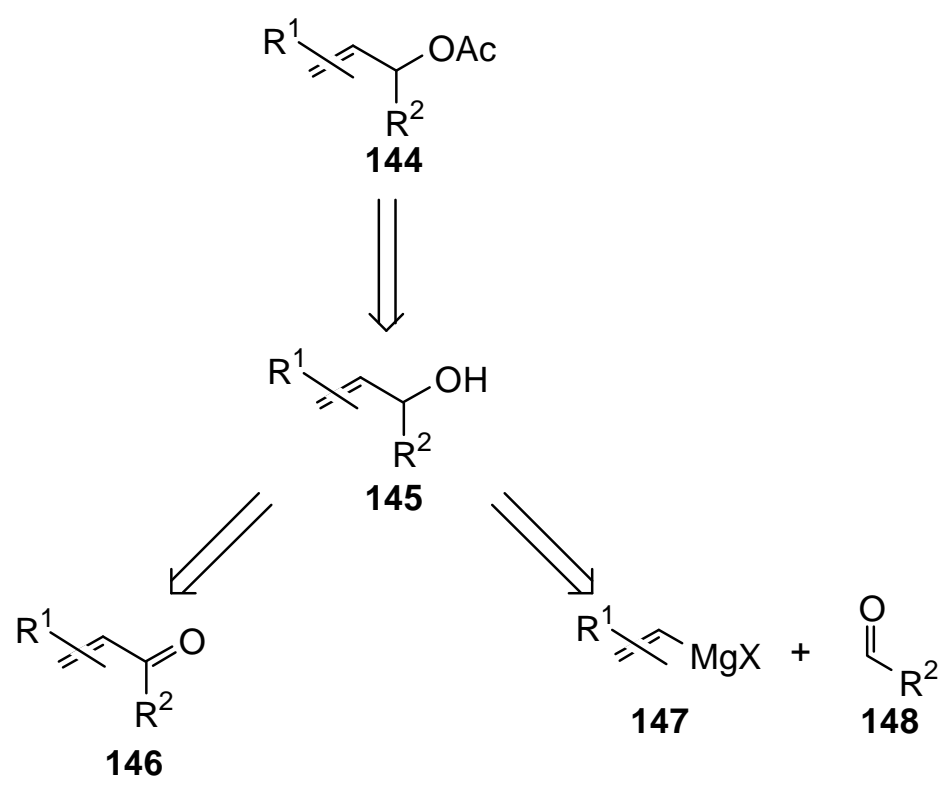

\subsubsection{Acetylierung von Allylalkoholen}

Die kommerziell erhältlichen Allylalkohole 149-154 beschränken sich im wesentlichen auf einfache, aliphatische Verbindungen, die sich in guten bis sehr guten Ausbeuten acetylieren ließen. Hierzu wurden sie mit Essigsäureanhydrid 155 in Gegenwart von Triethylamin und katalytischen Mengen 4-Dimethylaminopyridin in Dichlormethan umgesetzt. Man erhielt die Allylacetate 156-161 (Tabelle 1) mit hoher Reinheit, so daß sie ohne weitere Reinigung in den Allylierungsreaktionen eingesetzt werden konnten.

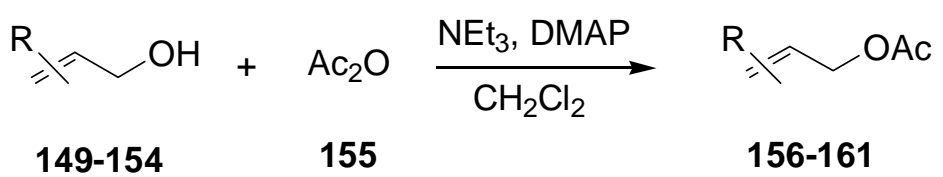




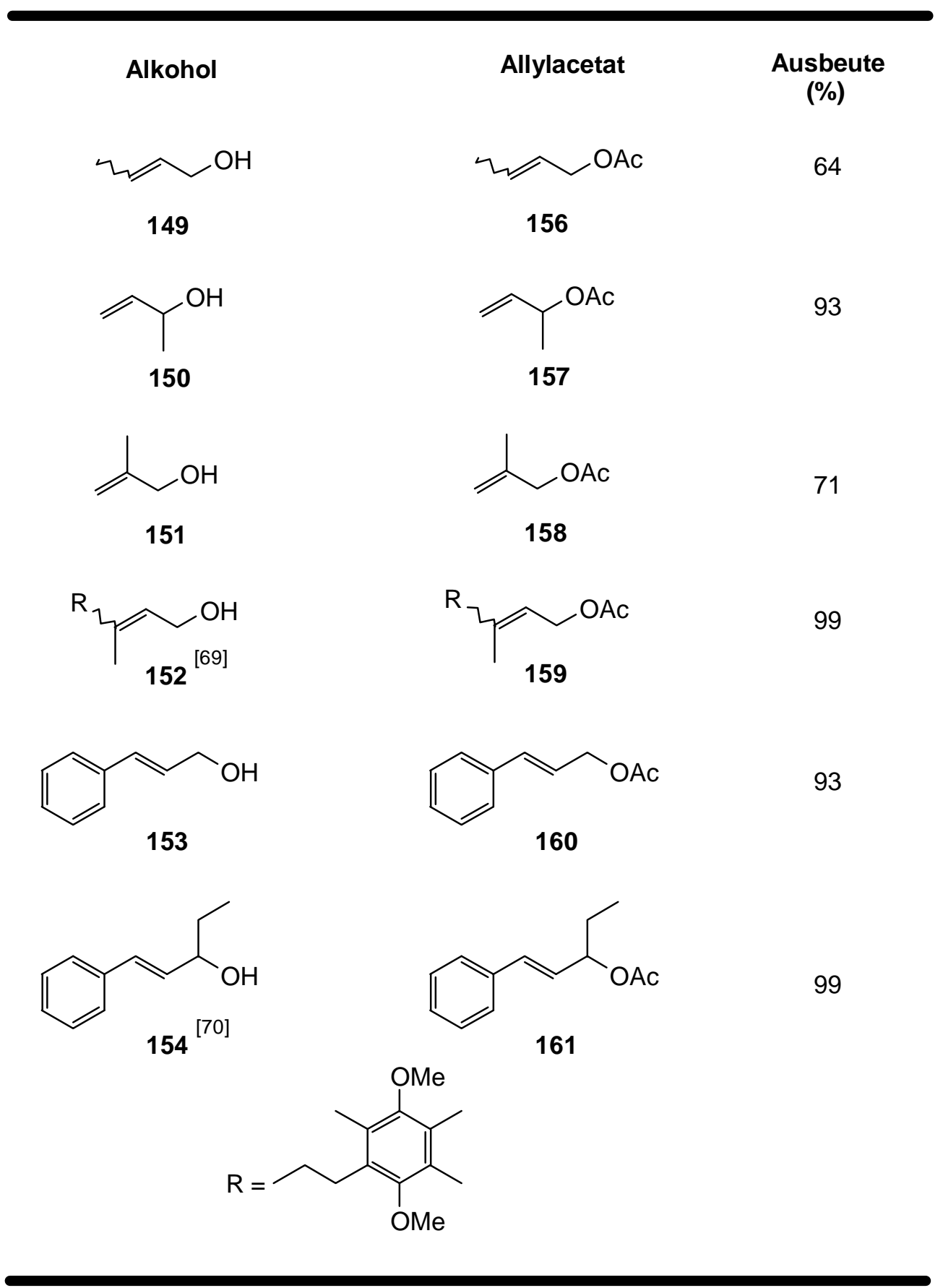

Tabelle 1: Synthetisierte Allylacetate 156-161.

\subsubsection{Reduktions-Acetylierungs-Sequenz}

Die Reduktion von $\alpha, \beta$-ungesättigten Carbonylverbindungen läßt sich problemlos und in hohen Ausbeuten durch Verwendung von Diisobutylaluminiumhydrid (DIBAH) 168 erreichen. So konnten die cyclischen, ungesättigten Ketone 162-165 und der Zimtaldehyd 166 bei $-78{ }^{\circ} \mathrm{C}$ zu den entsprechenden Alkoholen 169-173 umgesetzt werden. Die Reduktion des Zimtsäurederi- 
vats 167 wurde bei Raumtemperatur mit einem Überschuß an DIBAH durchgeführt. Trotzdem verlief die Reduktion aufgrund der geringen Reaktivität des intermediär entstehenden Carboxylat-Anions nicht vollständig.

Die erhaltenen Alkohole 169-174 ließen sich glatt zu den gewünschten Allylacetaten 175-180 umsetzen (Tabelle 2) und wurden ohne weitere Aufreinigung in den folgenden Reaktionen eingesetzt.

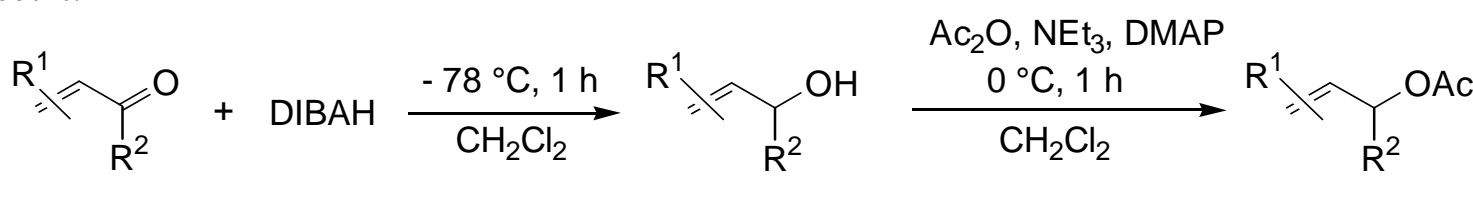

$162-167$

168

169-174

$175-180$

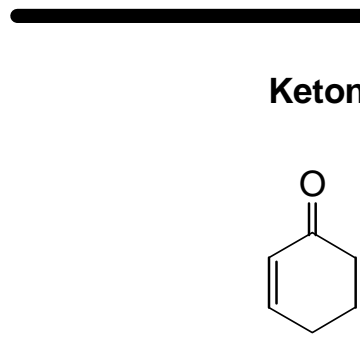

162<smiles>O=C1C=CCC1</smiles>

163<smiles>CC1=CCCC1=O</smiles>

164<smiles>CC1=CC(=O)CC1</smiles>

165
Allylacetat<smiles>CC(=O)OC1C=CCCC1</smiles>

175

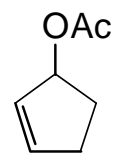

176<smiles>CC(=O)OC1CCC=C1C</smiles>

177<smiles>CC(=O)OC1C=C(I)CC1</smiles>

178
Ausbeute

(\%)

86

78

84

92

73

42<smiles>COc1cccc(/C=C/C(=O)O)c1</smiles>

Tabelle 2: Synthetisierte Allylacetate 175-180. 


\subsubsection{Grignard-Acetylierungs-Sequenz}

Ein weiterer kurzer Zugang zu Allylacetaten besteht in der Reaktion eines Aldehyds mit einer Vinyl-Grignard-Verbindung und nachfolgender Acetylierung. Auf diesem Wege wurden eine Reihe von heteroaromatischen Allylacetaten 190-193 synthetisiert (Tabelle 3).

Bei der Durchführung der Grignard-Reaktion wurde das Vinyl-Magnesium-Bromid 185 sehr langsam zur Reaktionslösung hinzugegeben, wodurch die Ausbeuten gesteigert werden konnten. Allerdings verliefen die Umsetzungen mit den Pyridincarbaldehyden 183 und 184 unter Auftreten von Nebenprodukten. In jedem Fall wurden die erhaltenen Rohprodukte unmittelbar in der nächste Reaktion eingesetzt, eine säulenchromatographische Reinigung erfolgte erst auf der Stufe der Allylacetate.
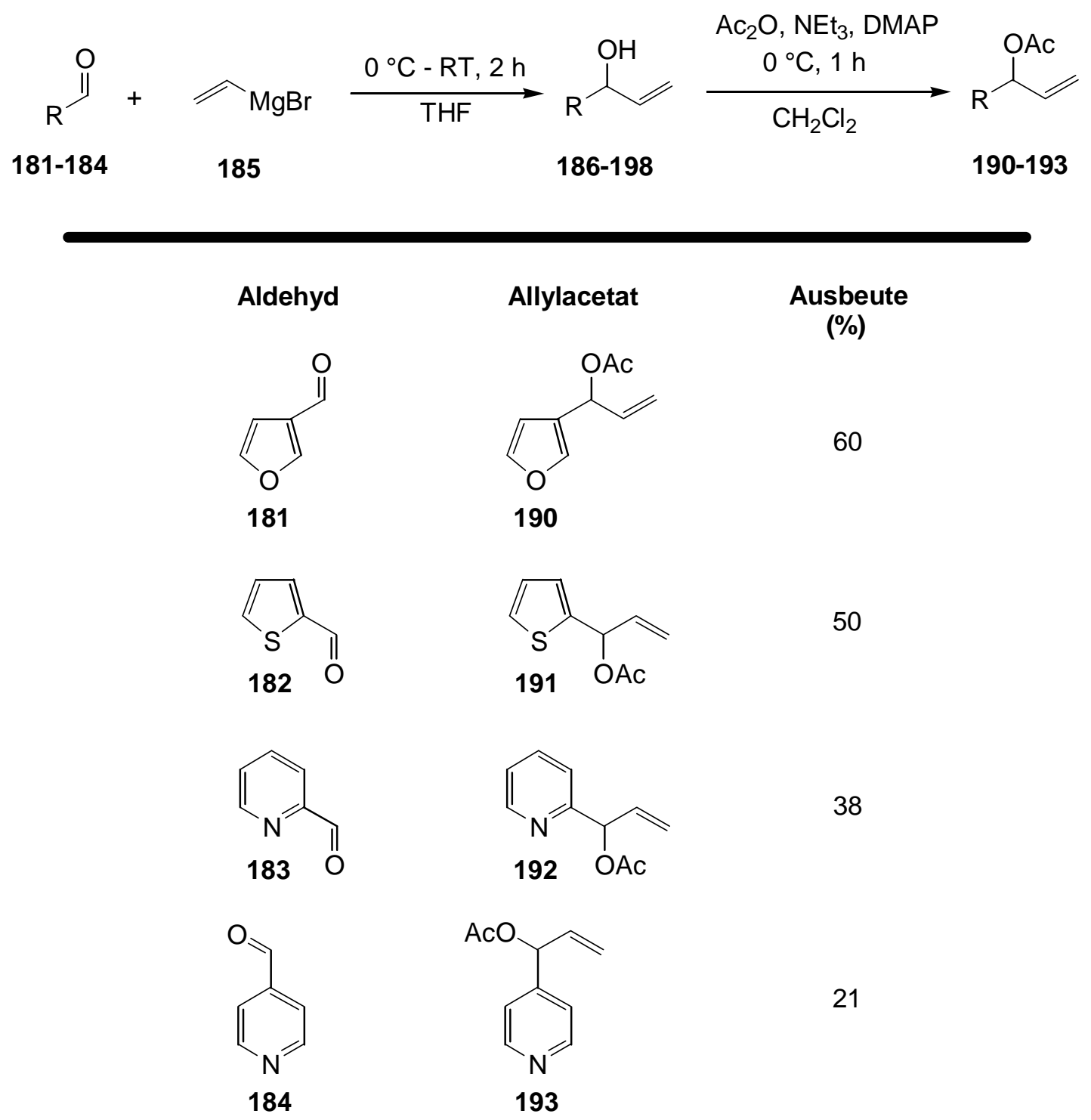

Tabelle 3: Synthetisierte Allylacetate 190-193. 


\subsection{Synthese der Allylcarbonate}

Zur Darstellung der Allylcarbonate 196 und 198 wurde auf die kommerziell erhältlichen Allylalkohole 194 und 197 zurückgegriffen, die mit Hilfe von Chlorameisensäure-ethylester 195 in die Zielverbindungen umgewandelt werden konnten.
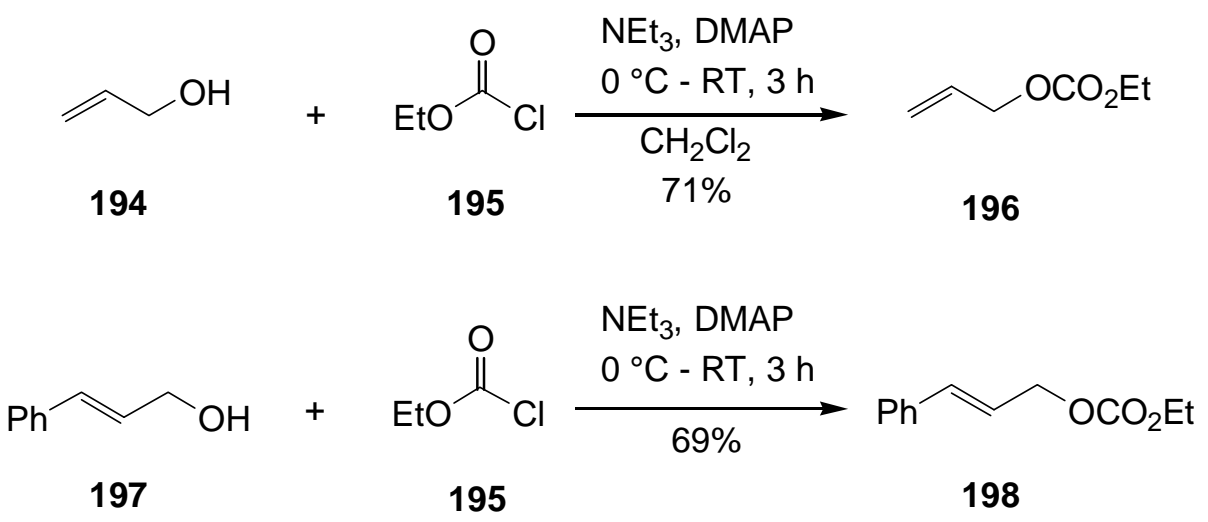


\section{Allylische Alkylierungen an der festen Phase}

Allylische Alkylierungen in flüssiger Phase sind eine seit langem bekannte, effektive Methode zum Aufbau von C-C- und C-X-Bindungen. ${ }^{[40]}$ Dabei wird in den meisten Fällen zunächst mit Hilfe von Natriumhydrid das Pronucleophil deprotoniert und dann mit der $\pi$-Allyl-PalladiumSpezies umgesetzt. Ist das Nucleophil jedoch polymergebunden, so muß die Deprotonierung mit einer flüssigen Base erfolgen, da das unlösliche Natriumhydrid nicht oder nur sehr unzureichend in den polymeren Träger hineindiffundieren kann. Eine elegante Lösung besteht in der Verwendung des bereits von B. M. Trost ${ }^{[61]}$ vorgeschlagenen und in allylischen Allylierungen in flüssiger Phase eingesetzten Basensystems N,O-Bis(trimethylsilyl)-acetamid (BSA)/Kaliumacetat. Dabei erfolgt durch Silylierung ${ }^{[61]}$ des Kaliumacetats in situ die Generierung der freien Base, die dann die 1,3-Dicarbonylverbindung deprotonieren kann.

\subsection{Verwendung aliphatischer Allylacetate}

Das unsubstituierte Allylacetat 199 konnte glatt mit polymergebundenem Acetoacetat 138 und Methylmalonat $143 \mathrm{zu}$ den entsprechenden festphasengebundenen Diallylverbindungen 200 und 201 umgesetzt werden. Es wurden 20 mol\% Tetrakis(triphenylphosphan)palladium als Katalysator und das oben angesprochene Basensystem BSA/KOAc verwendet und die Mischung in THF für 5 Stunden am Rückfluß erhitzt. Nach reduktiver Abspaltung von der festen Phase mit DIBAH konnten die 1,3-Diole 202 und 203 in Ausbeuten über drei Stufen von 88 bzw. 68\% ausgehend vom spacermodifizierten Merrifield-Harz 6 erhalten werden. Es wurden dabei ausschließlich die diallylierten Produkte gebildet, was auf die verwendeten Überschüsse von 5 Äquivalenten an Allylacetat zurückzuführen ist. Das Allylacetat 199 ist folglich unter den Reaktionsbedingungen zu reaktiv, um die Reaktion auf der Stufe der Monoalkylierung anzuhalten. Diese Beobachtung steht im Einklang mit analogen Umsetzungen in flüssiger Phase. ${ }^{[40]}$ 


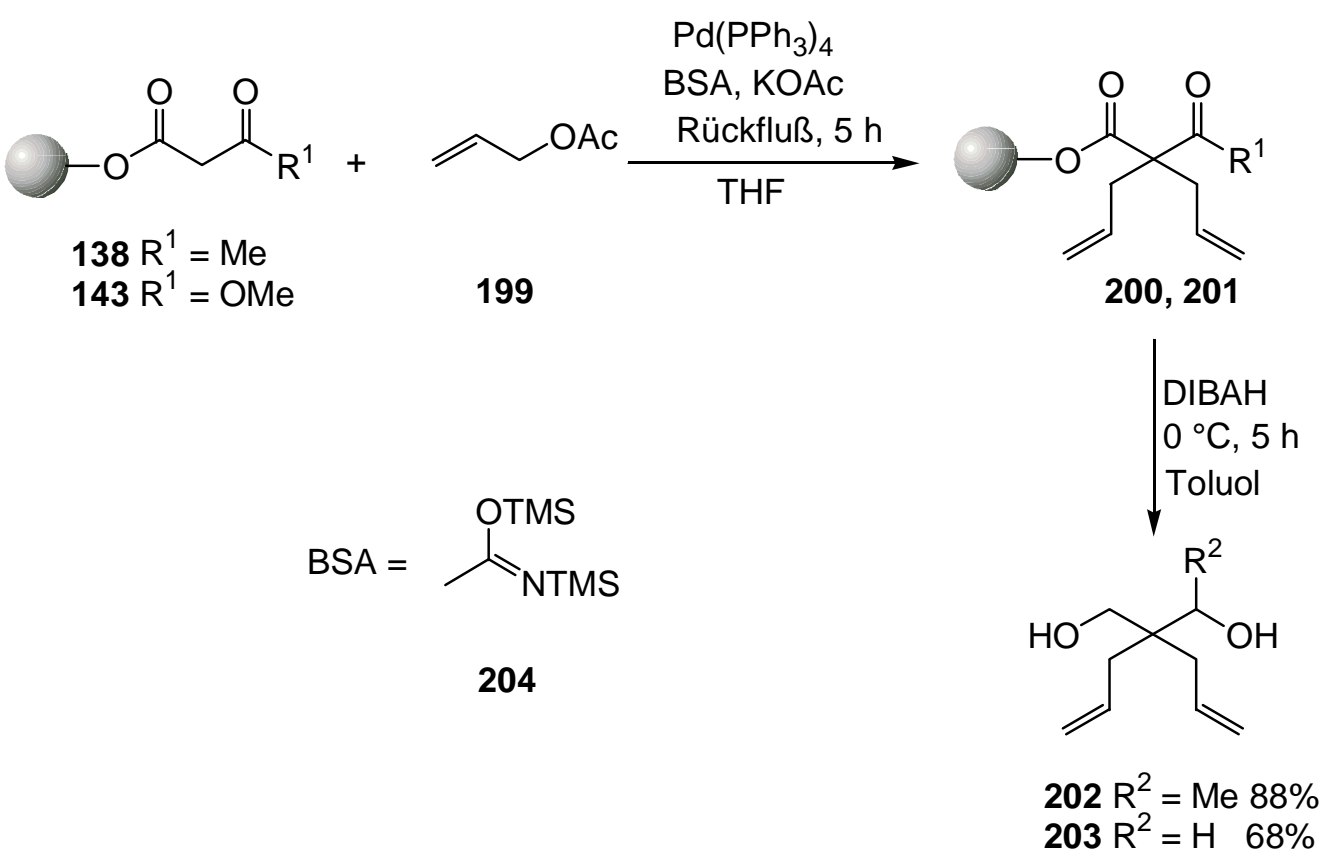

Die Menge des eingesetzten Palladium-Katalysators konnte bei Reaktionen an fester Phase nicht reduziert werden. Versuchte Synthesen mit nur 10 mol\% Tetrakis(triphenylphosphan)palladium ergaben unvollständige Umsetzungen in schlechten Ausbeuten nach Abspaltung von der festen Phase. Es muß angenommen werden, daß ein Teil des Palladium-Komplexes durch das Polystyrol-Harz desaktiviert wird und somit nicht zur Katalyse der Allylierung zur Verfügung steht. In flüssiger Phase genügten 7 mol\% Katalysator für die Synthese von 206 innerhalb von 30 Minuten unter Erhitzen am Rückfluß.<smiles>COC(=O)CC(C)=O</smiles>

205

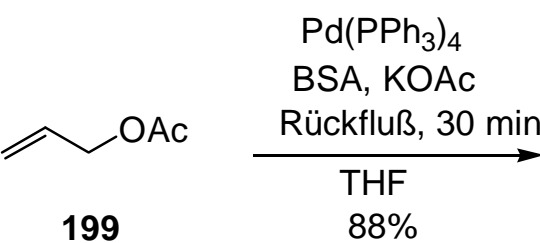

199<smiles>C=CCC(CC=C)(C(C)=O)C(=O)OC</smiles>

206

Eine Reihe weiterer aliphatischer Allylacetate wurden unter den oben erprobten Standardbedingungen (5 Äquivalente Allylacetat, 20 mol\% Tetrakis(triphenylphosphan)palladium, 5 Äquivalente BSA, kat. KOAc, Rückfluß, THF) mit polymergebundenem Acetoacetat 138 und Methylmalonat 143 umgesetzt. Dabei wurden verschiedene Substitutionsmuster der AllylKomponente ausgewählt, durch die die Bildung der $\pi$-Allyl-Palladium-Spezies und der Angriff des polymergebundenen Nucleophils erschwert werden. Neben dem unsubstituierten Palladium-Komplex 214 setzte man den terminal-substituierten Komplex 215 und den internsubstituierten Komplex 216 sowie den 1,1-disubstituierten Komplex 217 ein. 


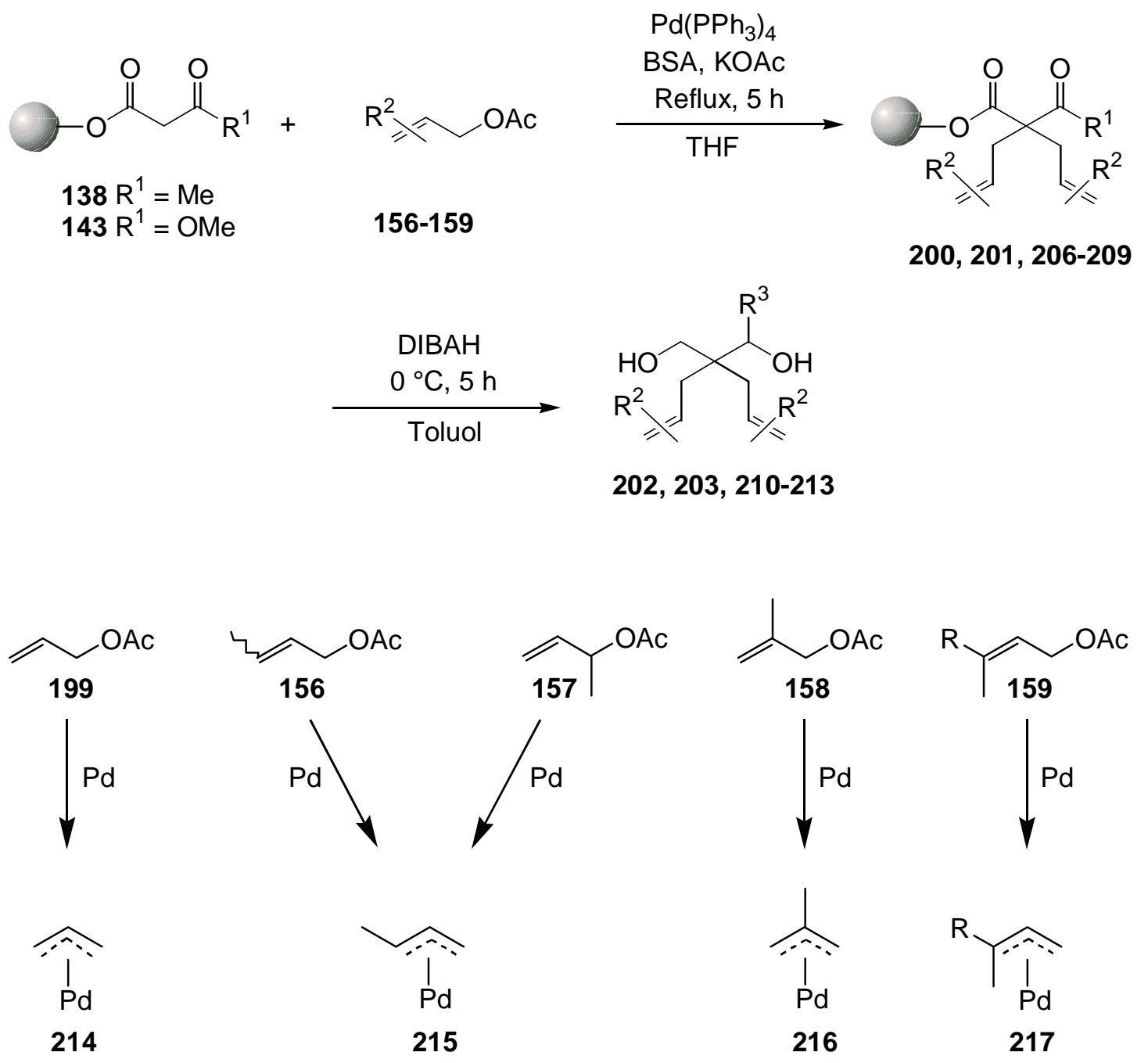<smiles>[2H]CCc1c(C)c(C)c(C)c(OC)c1C</smiles>

Wie aus Tabelle 4 ersichtlich sanken die Ausbeuten der erhaltenen 1,3-Diole 210-213 infolge der Substitution der $\pi$-Allyl-Palladium-Spezies, wobei eine Methyl-Gruppe in 1-Position einen weitaus größeren Effekt als in 2-Position hat. Besonders bei Einsatz des 1,1-disubstituierten $\pi$ Allyl-Palladium-Komplexes 217 sank die Ausbeute drastisch. Hier erfolgte auch nur eine Monoalkylierung des polymergebundenen Methylmalonats 143, was die eingeschränkte Reaktivität der Spezies 217 unterstreicht. 
Allylacetat<smiles>C=CCOC(C)=O</smiles>

199<smiles></smiles>

156<smiles>C=CC(C)OC(C)=O</smiles>

157<smiles>C=C(C)COC(C)=O</smiles>

158<smiles>[R17]C(C)=CCOC(C)=O</smiles><smiles>COc1c(C)c(C)c(CC[18F])c(C)c1OC</smiles>

Produkt<smiles>[R]C(O)C(CC=C)(CC=C)CCO</smiles><smiles>[R]C(O)C(CO)(C/C=C/C)C/C=C/C</smiles><smiles>[R]C(O)C(CO)(C/C=C\C)C/C=C/C</smiles><smiles>[R]C(O)C(CO)(CO)CC(=C)C</smiles><smiles>[Z7]C(C)=CCC(CO)C([R])O</smiles>

$203 \mathrm{R}=\mathrm{H}$

$210 R=M e$

$210 R=M e$

39

$211 \mathrm{R}=\mathrm{Me}$

$212 \mathrm{R}=\mathrm{H}$

60 45

Ausbeute

(\%)

88

68

39

$213 \mathrm{R}=\mathrm{Me}$

8

Tabelle 4: Synthetisierte 1,3-Diole 202, 203, 210-213.

In allen Fällen wurden ausschließlich die $E$-Verbindungen erhalten, unabhängig von der Ausgangskonfiguration des Allylacetats. Ursache hierfür ist der angenommene $\pi$ - $\sigma-\pi$ Mechanismus (siehe Kapitel 3.1.), der eine schnelle Isomerisierung der terminalen Substituenten der $\pi$-Allyl-Palladium-Spezies bewirkt, so daß 156 und 157 beide zu Komplex 215 führen und identische Produkte liefern. Man erkennt außerdem, daß die $\pi$-Allyl-Palladium-Spezies 
bevorzugt am weniger substituierten Ende nucleophil von der polymergebundenen 1,3Dicarbonylverbindung angegriffen wird, was schon aus der Chemie in flüssiger Phase bekannt ist. $^{[40 a]}$

Neben dem polymergebundenem Acetoacetat 138 können auch $\gamma$-substituierte $\beta$-Ketoester für allylische Alkylierungen an der festen Phase eingesetzt werden. Dies wurde am Beispiel des festphasengebundenen Phenylacetoacetats $\mathbf{2 1 8}^{[62]}$ gezeigt, welches unter den Standardbedingungen mit Allylacetat 199 umgesetzt wurde. Nach reduktiver Abspaltung von der festen Phase erhielt man das gewünschte 1,3-Diol 219 in einer Gesamtausbeute von $73 \%$.

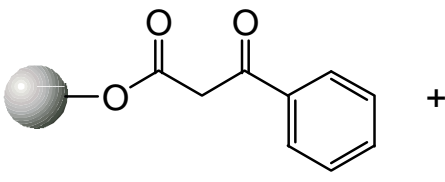

218

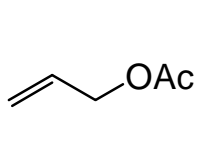

199
1) $\mathrm{Pd}\left(\mathrm{PPh}_{3}\right)_{4}$ BSA, KOAc Rückfluß, 5h, THF

2) DIBAH, Toluol $73 \%$

\subsubsection{Diskussion ausgewählter spektroskopischer Daten}

Aufgrund der strukturellen Ähnlichkeit der 1,3-Diole 202, 203 und 210-213 werden nur die spektroskopischen Daten der Verbindungen 202 und 203 im Detail diskutiert.

Im ${ }^{1} \mathrm{H}$-NMR-Spektrum von $\mathbf{2 0 3}$ erkennt man bei $\delta=2.08$ das Signal der beiden $\mathrm{CH}_{2}$-Gruppen der Allyleinheiten, das als Dublett mit einer Kopplungskonstanten von $J=7.0 \mathrm{~Hz}$ aufspaltet. Die beiden $\mathrm{OH}-\mathrm{Gruppen}$ resonieren als breites Singulett bei $\delta=2.74$. Das Signal der MethylenGruppen der Propandiol-Einheit beobachtet man bei $\delta=3.58$ als Singulett. Die trans-ständigen Protonen der Allyl-Gruppen resonieren bei $\delta=5.07$ als Dublett mit einer cis-Kopplung von $J=11.8 \mathrm{~Hz}$ zu den 2'- und 2' '-Protonen, während das Signal der cis-ständigen Protonen bei $\delta=5.10$ als Dublett mit einer Kopplungskonstanten von $J=15.6 \mathrm{~Hz}$ aufspaltet. Die Protonen der Methin-Gruppen resonieren als Dublett vom Dublett vom Triplett bei $\delta=5.84$ mit Kopplungen zu den terminalen Protonen mit $J=15.6 \mathrm{~Hz}$ bzw. $J=11.8 \mathrm{~Hz}$ sowie einer Kopplung zu den beiden 1'- und 1' '- $\mathrm{CH}_{2}$-Gruppen mit $J=7.0 \mathrm{~Hz}$.

Das ${ }^{13}$ C-NMR-Spektrum zeigt im hohen Feld das Signal der Methylen-Gruppen der Allyleinheiten bei $\delta=19.90$. Die Resonanz des quartären C-2-Atoms erkennt man bei $\delta=42.05$, während C-1 und C-3 bei $\delta=68.13$ resonieren. Die Methylen-Signale der Doppelbindungen findet man bei $\delta=118.1$, die der Methin-Gruppen bei $\delta=133.8$.

Im IR-Spektrum erkennt man bei $3384 \mathrm{~cm}^{-1}$ die breite Bande der OH-Gruppen, bei 2952 und $2927 \mathrm{~cm}^{-1}$ zeigen sich die C-H-Streckschwingungen. Die Bande bei $1639 \mathrm{~cm}^{-1}$ kann den olefinischen Bindungen zugeordnet werden. 
Das DCI-Massenspektrum zeigt die Massenpeaks $\mathrm{M}+18, \mathrm{M}+18+17$ und $2 \mathrm{M}+18$ bei $\mathrm{m} / \mathrm{z}=$ 174, 191 und 330.

202 zeigt ein deutlich komplexeres ${ }^{1}$ H-NMR-Spektrum als 203, da es aufgrund der zusätzlichen Methyl-Gruppe nicht mehr $\mathrm{C}_{2}$-symmetrisch ist. Die Protonen dieser $\mathrm{CH}_{3}$-Gruppe resonieren bei $\delta=1.26$ als Dublett mit $J=6.2 \mathrm{~Hz}$. Die Signale der diastereotopen Protonen der 1'$\mathrm{CH}_{2}$-Gruppe erscheinen als Dublett vom Dublett bei $\delta=1.92$ und $\delta=2.19$ und zeigen eine Kopplung zu der 2'-CH-Gruppe mit $J=7.4 \mathrm{~Hz}$ und eine geminale Kopplung untereinander mit $J=14.1 \mathrm{~Hz}$. Die 1' $-\mathrm{CH}_{2}$-Gruppe resoniert als Dublett mit $J=7.4 \mathrm{~Hz}$ bei $\delta=2.28$ und die beiden $\mathrm{OH}-G r u p p e n$ als breites Singulett bei $\delta=2.35$. Die Resonanzen der diastereotopen 1- $\mathrm{CH}_{2}$-Protonen zeigen sich jeweils als Dublett mit einer geminalen Kopplung von $J=11.0 \mathrm{~Hz}$ bei $\delta=3.57$ bzw. $\delta=3.75$. Als Quartett mit $J=6.2 \mathrm{~Hz}$ beobachtet man das Signal des Protons an C-3. Die Signale der trans-ständigen Protonen der Allyl-Gruppen resonieren bei $\delta=5.08$ als Dublett mit einer cis-Kopplung von $J=11.5 \mathrm{~Hz}$, während die cis-ständigen Protonen bei $\delta=5.12$ mit $J=15.1 \mathrm{~Hz}$ aufspalten. Das Signal von 2'-H und 2' '-H erscheint bei $\delta=5.86$ als Dublett vom Dublett vom Triplett mit $J=15.1,11.5$ und $6.2 \mathrm{~Hz}$.

Das ${ }^{13} \mathrm{C}-\mathrm{NMR}-$ Spektrum von 202 weist bei $\delta=18.04$ das Signal von C-4 auf. Die MethylenKohlenstoffatome der Allyleinheiten resonieren bei $\delta=36.14$, während das quartäre C-2 ein Signal bei $\delta=45.47$ erzeugt. Die Resonanz von C- 1 bzw. C-3 erkennt man bei $\delta=67.03$ bzw. 73.87. Das Signal der terminalen olefinischen Kohlenstoffatome zeigt sich bei $\delta=118.1$, das der internen bei $\delta=133.9$.

Im IR-Spektrum erkennt man bei $3350 \mathrm{~cm}^{-1}$ die Bande der OH-Gruppen. Die C-H-Streckschwingungen zeigen sich bei 3005 und $2958 \mathrm{~cm}^{-1}$, während die Banden bei 1451 und $1378 \mathrm{~cm}^{-1}$ als $\mathrm{CH}_{3}$-Deformationsschwingungen interpretiert werden können. Eine olefinische Bande erkennt man bei $1642 \mathrm{~cm}^{-1}$.

Das DCI-Massenspektrum zeigt die Massenpeaks M+18 und M+18+17 m/z = 188 und 205.

\subsection{Verwendung cyclischer Allylacetate}

Die cyclischen Allylacetate 175-178 wurden mit polymergebundenem Acetoacetat 138 und Methylmalonat 143 unter den erprobten Bedingungen umgesetzt. Dabei wurden selektiv die monoalkylierten Verbindungen in Gesamtausbeuten von 14 - 59\% erhalten (Tabelle 5). Selbst bei deutlich längeren Reaktionszeiten und größeren Überschüssen an Allylacetat konnte keine Dialkylierung beobachtet werden, so daß die allylische Alkylierung mit cyclischen Allylacetaten eine gute synthetische Methode darstellt, monoalkylierte 1,3-Dicarbonylverbindungen an der festen Phase zu generieren. 


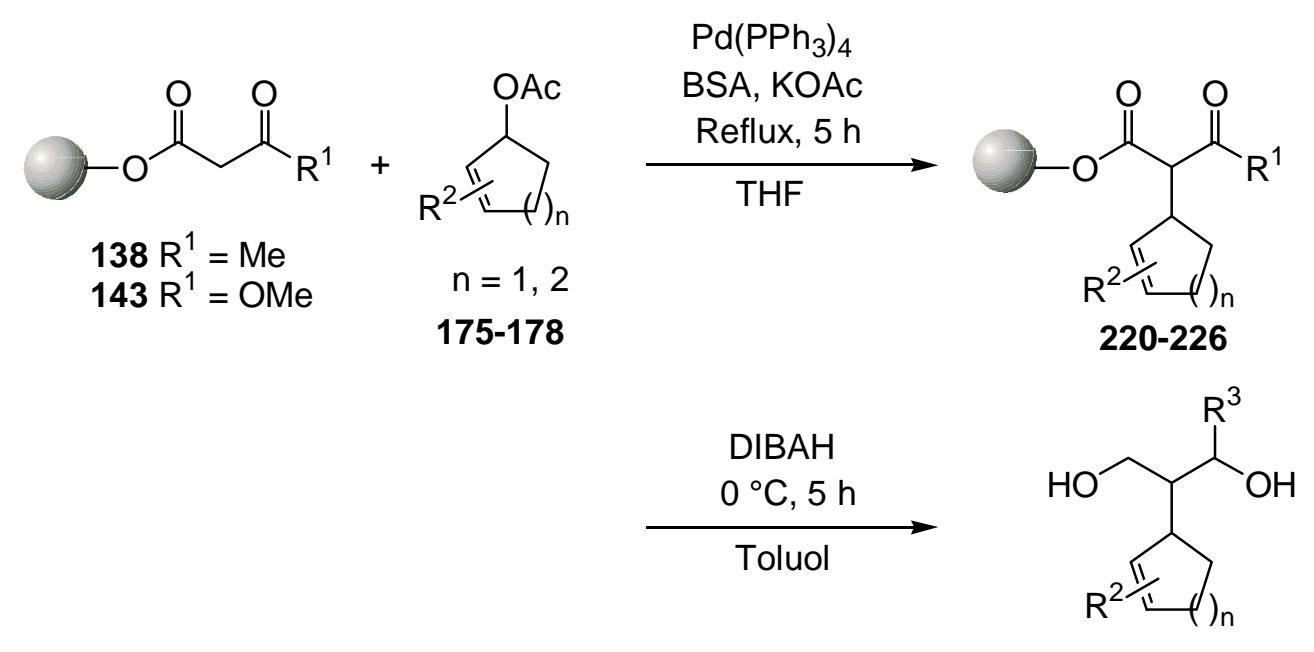

227-233

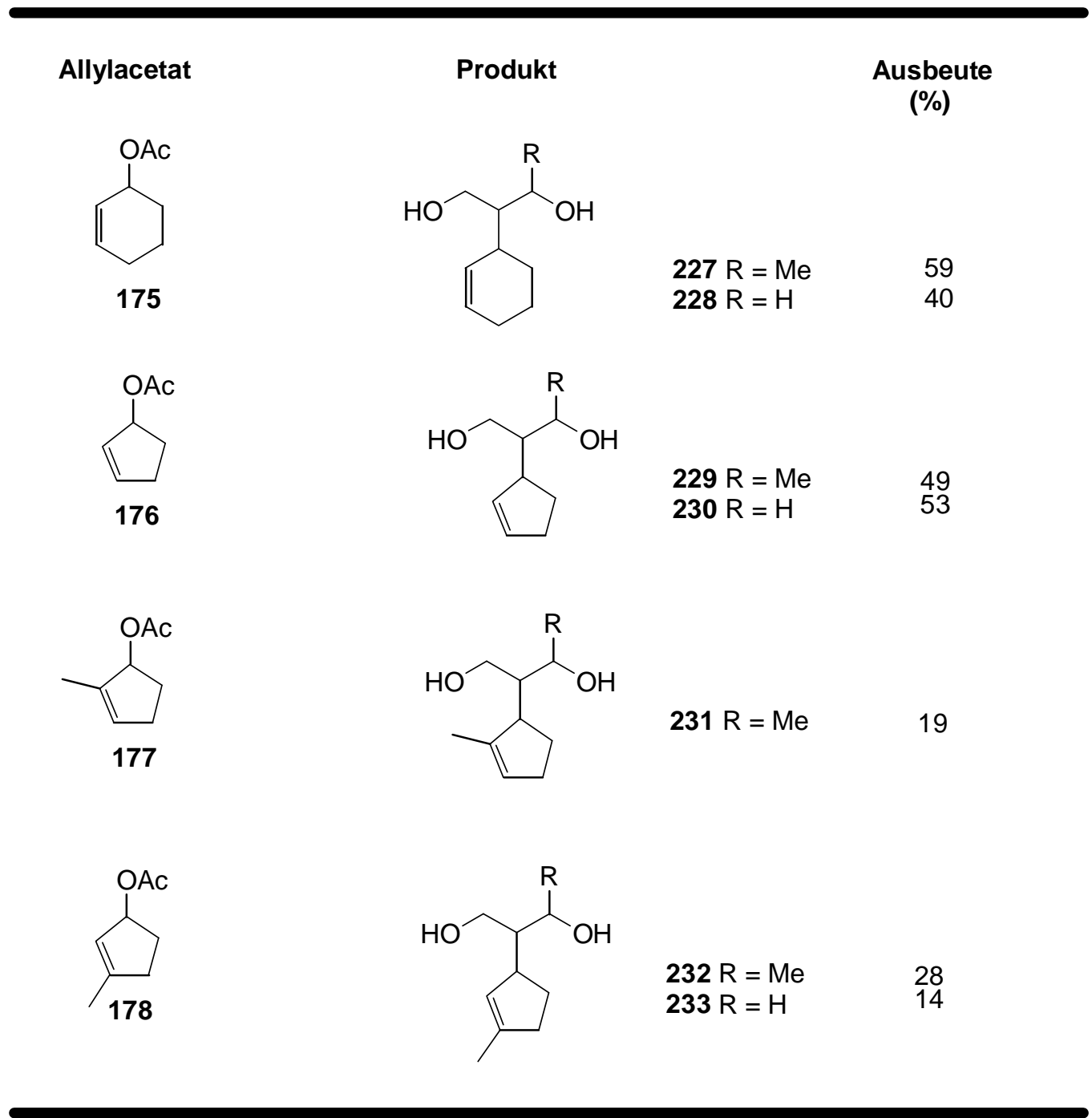

Tabelle 5: Synthetisierte 1,3-Diole 227-233.

Es zeigt sich, daß die unsubstituierten Cyclopentenyl- und Cyclohexenylacetate gute Substrate für die allylische Alkylierung an der festen Phase darstellen. An der Doppelbindung substitu- 
ierte Cyclopentenyl- und Cyclohexenylacetate konnten jedoch nur in geringeren Ausbeuten alkyliert werden.

\subsubsection{Diskussion ausgewählter spektroskopischer Daten}

Im ${ }^{1}$ H-NMR-Spektrum der Verbindung 227 erkennt man bei $\delta=1.30$ das Signal der MethylGruppe als Dublett mit einer Kopplungskonstanten von $J=5.9 \mathrm{~Hz}$. Aufgrund der Flexibilität des Ringes beobachtet man die Resonanzen von 2- $\mathrm{H}, 1^{\prime}-\mathrm{H}, 5^{\prime}-\mathrm{H}_{2}$ und 6'- $\mathrm{H}_{2}$ als Multiplett bei $\delta=1.34-1.84$ und die von 4'- $\mathrm{H}_{2}$ als zentriertes Multiplett bei $\delta=1.99$. Die OH-Gruppen ergeben ein Signal bei $\delta=2.46$ als breites Singulett. Ein zentriertes Multiplett bei $\delta=3.82$ kann der Methylen-Gruppe der Butandiol-Einheit zugeordnet werden, während deren MethinGruppe bei $\delta=4.06-4.21$ als Multiplett resoniert. Die Protonen der Doppelbindung ergeben ein Multiplett-Signal bei $\delta=5.56-5.86$.

Das ${ }^{13} \mathrm{C}-\mathrm{NMR}-$ Spektrum von 227 zeigt bei $\delta=22.00,24.90$ und 25.25 die Signale von C-4', C-5' und C-6'. Die Methyl-Gruppe resoniert bei $\delta=22.57$ und das quartäre C-2 bei $\delta=50.63$. Die Signale von C-1 und C-3 finden sich bei $\delta=62.78$ bzw. $\delta=70.20$, während die Doppelbindungskohlenstoffatome bei $\delta=128.5$ und $\delta=128.6$ resonieren.

Das IR-Spektrum zeigt bei $3377 \mathrm{~cm}^{-1}$ die Bande der OH-Gruppen und bei 2968 und $2931 \mathrm{~cm}^{-1}$ die C-H-Streckschwingungen. Bei $1652 \mathrm{~cm}^{-1}$ erkennt man die Bande der olefinischen Bindungen und bei 1453 und $1378 \mathrm{~cm}^{-1}$ die $\mathrm{CH}_{3}$-Deformationsschwingungen.

Das DCI-Massenspektrum zeigt die Massenpeaks M+18, M+18+17 und $2 \mathrm{M}+18$ bei $\mathrm{m} / \mathrm{z}=$ 188,205 und 358.

Im ${ }^{1} \mathrm{H}-\mathrm{NMR}$-Spektrum von 230 resoniert das $2-\mathrm{H}$ bei $\delta=1.45-1.65$ ebenso als Multiplett wie das 1 '-H bei $\delta=1.67-1.85$. Ein zentriertes Multiplett bei $\delta=1.76$ läßt sich dem 5 ' $-\mathrm{H}_{2}$ und ein breites Singulett bei $\delta=2.31$ den beiden OH-Gruppen zuordnen. Bei $\delta=2.74$ resoniert das 4'$\mathrm{H}_{2}$ als zentriertes Multiplett und bei $\delta=3.71$ - 3.95 die $\mathrm{CH}_{2}$-Gruppen der Propandiol-Einheit ebenfalls als Multiplett. Im Tieffeld läßt sich schließlich bei $\delta=5.66$ - 5.84 das MultiplettSignal der olefinischen Protonen erkennen.

Im ${ }^{13}$ C-NMR-Spektrum zeigen sich bei $\delta=27.76$ bzw. bei $\delta=32.04$ die Signale von C-5' bzw. C-4'. C-1' resoniert bei $\delta=44.02$ und das quartäre C-2 bei $\delta=46.79$, während die Signale von C-1 und C-3 bei $\delta=65.41$ und bei $\delta=65.63$ zu finden sind. Die Resonanz der Doppelbindungskohlenstoffatome erkennt man bei $\delta=131.8$ und bei $\delta=132.1$.

Das IR-Spektrum zeigt bei $3355 \mathrm{~cm}^{-1}$ die Bande der OH-Gruppen und bei 2934 und $2889 \mathrm{~cm}^{-1}$ die C-H-Streckschwingungen. Bei $1652 \mathrm{~cm}^{-1}$ erkennt man die Bande der olefinischen Bindungen. 
Das DCI-Massenspektrum zeigt die Massenpeaks $\mathrm{M}+18, \mathrm{M}+18+17$ und $2 \mathrm{M}+18$ bei $\mathrm{m} / \mathrm{z}=$ 160, 176 und 302.

\subsection{Verwendung aromatischer und heteroaromatischer Allylacetate}

Das phenylsubstituierte Allylacetat 160 ließ sich in guter Gesamtausbeute von 52 bzw. 57\% mit polymergebundenem Acetoacetat 138 bzw. Methylmalonat 143 umsetzen. Durch die hohe Reaktivität der phenylsubstituierten $\pi$-Allyl-Palladium-Spezies und die verwendeten Überschüsse erhielt man selektiv die dialkylierten Verbindungen. Die Verwendung ElektronenDonor-substituierter Phenylallylacetate wie 179 und 180 ließ erwartungsgemäß die Ausbeute der synthetisierten 1,3-Diole 241-244 infolge des erschwerten Angriffs der KohlenstoffNucleophile auf den elektronenreicheren $\pi$-Allyl-Palladium-Komplex sinken. Eine weitere Reaktivitätsabsenkung erfolgt bei Einführung eines zusätzlichen Substituenten wie in 245, so daß in diesem Fall trotz Einsatz des Allylacetats im Überschuß lediglich die monoalkylierte Verbindung 246 erhalten wurde (Tabelle 6).
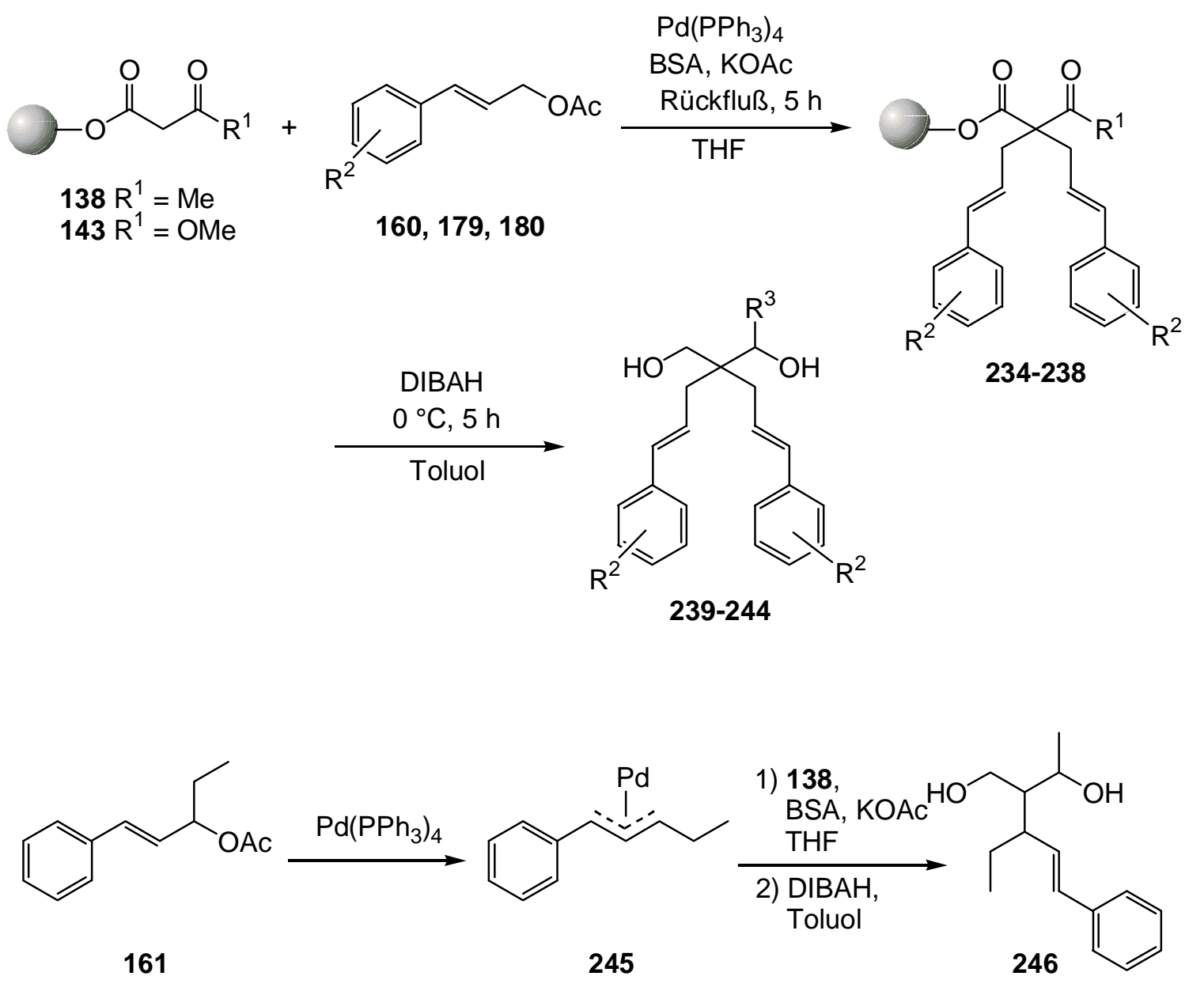


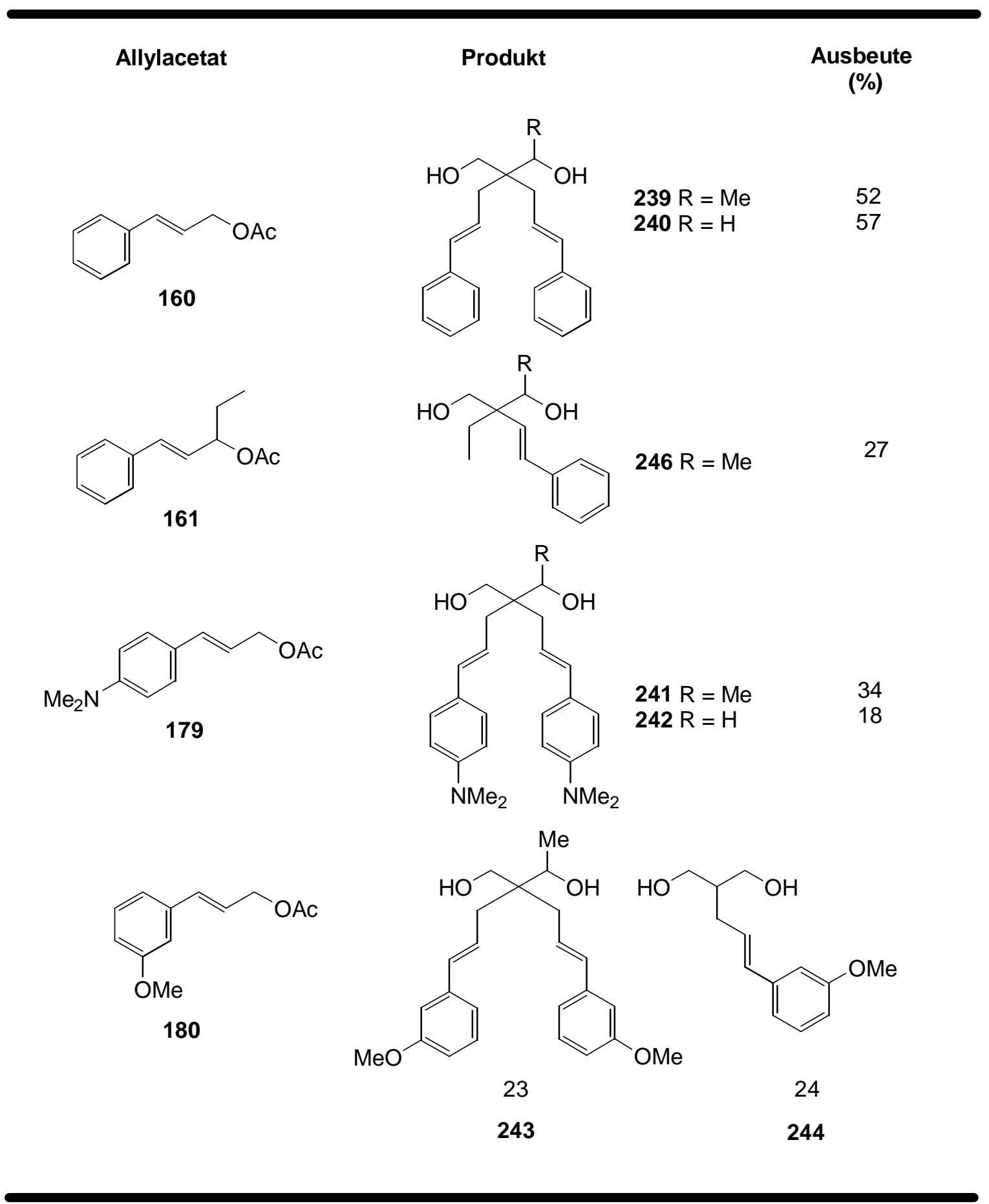

Tabelle 6: Synthetisierte 1,3-Diole 240-244, 246.

Einen überraschenden Effekt beobachtete man bei der Umsetzung des 3-Methoxysubstituierten Phenylallylacetats 180 mit festphasengebundenem Methylmalonat 143, die das monoalkylierte Diol 244 lieferte, während man bei Verwendung von polymergebundenem Acetoacetat 138 das erwartete dialkylierte Produkt $\mathbf{2 4 3}$ erhielt. Dies ist der einzige Fall, bei 
dem sich die beiden an die feste Phase gebundenen 1,3-Dicarbonylverbindungen in ihrem Reaktionsverhalten unterschieden.

Neben den aromatischen Allylacetaten 160, 161, 179, 180 wurden auch die heteroaromatisch substituierten Allylacetate 190 und 191 zur allylische Alkylierung an der festen Phase eingesetzt. Unter den verwendeten Standardbedingungen erhielt man selektiv die dialkylierten 1,3Diole 251-254 mit jeweils E-konfigurierter Doppelbindung (Tabelle 7). Wiederum lagen die erzielten Gesamtausbeuten von 23 - 14\% wegen des erschwerten Angriffs auf den elektronenreichen $\pi$-Allyl-Palladium-Komplex niedriger als bei Umsetzungen mit 160. Die erhaltenen Diole 251-254 wiesen darüber hinaus eine hohe Säureempfindlichkeit auf, die schon während der NMR-spektroskopischen Untersuchung durch Säurespuren im verwendeten Deuterochloroform zur Bildung von Zersetzungsprodukten führte.

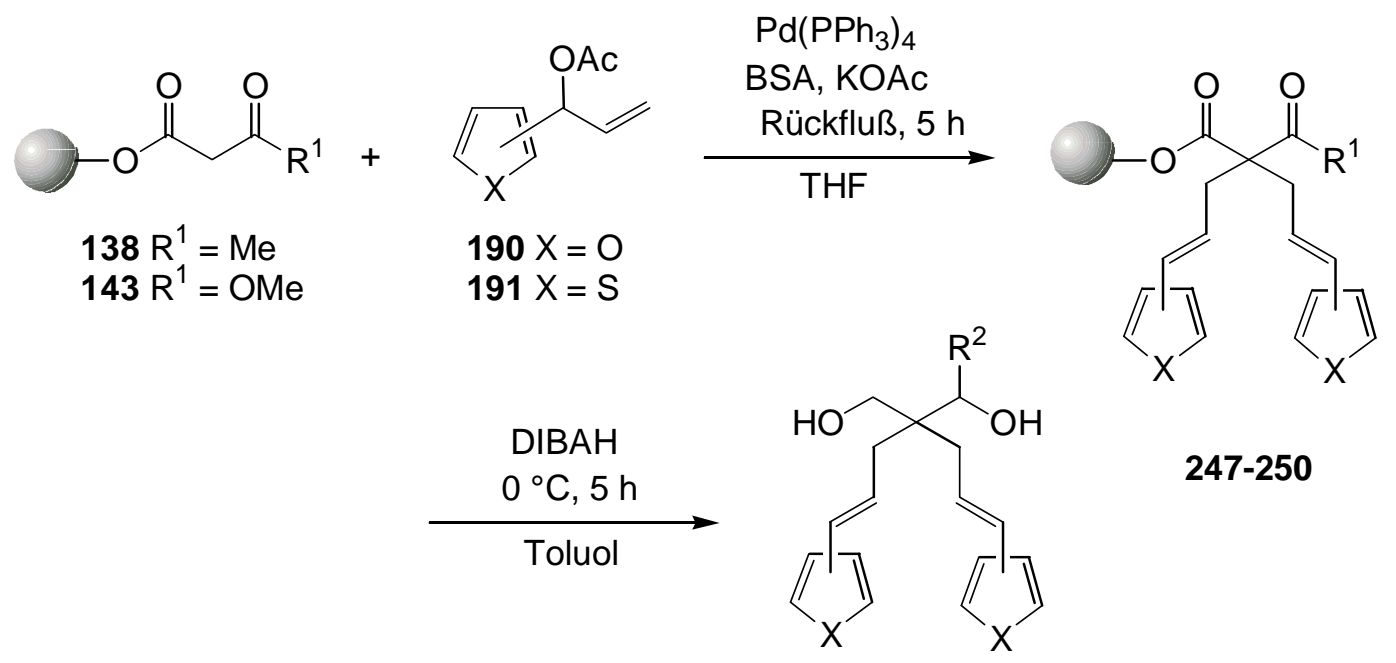

251-254 
Allylacetat

Produkt<smiles>C=CC(OC(C)=O)c1ccoc1</smiles><smiles>C=CC(OC(C)=O)c1cccs1</smiles>

191<smiles>[R]C(O)C(CO)(C/C=C/c1ccoc1)C/C=C/c1ccoc1</smiles>

$251 \mathrm{R}=\mathrm{Me} \quad 23$

$252 \mathrm{R}=\mathrm{H} \quad 20$

$253 \mathrm{R}=\mathrm{Me} \quad 20$

$254 \mathrm{R}=\mathrm{H} \quad 14$

Ausbeute

(\%)

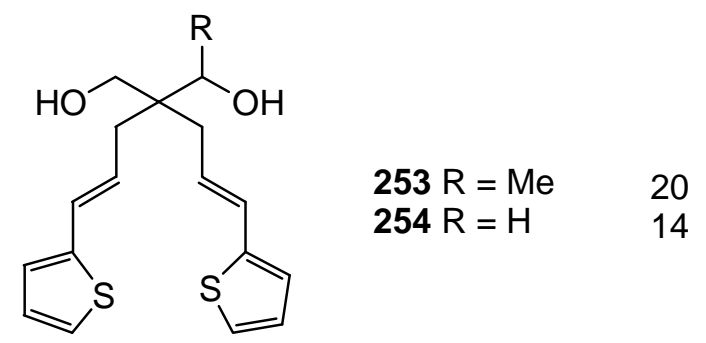

Tabelle 7: Synthetisierte 1,3-Diole 251-254.

Die Umsetzung des Pyridin-substituierten Allylacetats 192 mit polymergebundenem Acetoacetat 138 gelang nicht. Eine möglich Inhibierung der Reaktion durch intramolekulare Koordination des Palladiums an den Pyridin-Stickstoff in der intermediären $\pi$-Allyl-Palladium-Spezies konnte durch Einsatz des Allylacetats 193 ausgeschlossen werden. Eine mögliche intermolekulare Komplex-Bildung behindert ebenfalls nicht die Reaktion wie der Zusatz von 5 Äquivalenten Pyridin bei der Allylierung von Acetoacetat mit Allylacetat 199 an der festen Phase bewies, bei der das erwünschte, dialkylierte Produkt in einer Ausbeute von $86 \%$ erhalten wurde.<smiles>C=CC(OC(C)=O)c1ccccn1</smiles>

192<smiles>C=CC(OC(C)=O)c1ccncc1</smiles><smiles>CC(=O)CC(=O)Oc1ccccc1</smiles>

138

1) $\mathrm{Pd}\left(\mathrm{PPh}_{3}\right)_{4}$ $\underset{\text { 2) DIBA, KOAc, THF }}{\text { Doluol }}$ 


\subsubsection{Diskussion ausgewählter spektroskopischer Daten}

Im hohen Feld des ${ }^{1}$ H-NMR-Spektrums der Verbindung 239 resoniert die Methyl-Gruppe bei $\delta=1.31$ mit einer Kopplungskonstanten von $J=6.3 \mathrm{~Hz}$. Die Resonanzen der diastereotopen Protonen der 1'- $\mathrm{CH}_{2}$-Gruppe erkennt man bei $\delta=2.09$ bzw. bei $\delta=2.37$ jeweils als Dublett vom Dublett mit $J=14.2$ und 7.5 Hz, während die 1"'- $\mathrm{CH}_{2}$-Gruppe als Dublett-Signal mit $J=7.5 \mathrm{~Hz}$ bei $\delta=2.46$ erscheint. Die beiden OH-Protonen resonieren als Singulett bei $\delta=2.86$. Zwei Dublett-Signale bei $\delta=3.65$ und bei $\delta=3.83$ mit $J=10.7 \mathrm{~Hz}$ können der diastereotopen Methylen-Gruppe der Butandiol-Einheit zugeordnet werden, die Methin-Gruppe resoniert als Quartett mit $J=6.3 \mathrm{~Hz}$ bei $\delta=3.97$. Die Signale der 2'- und 2' '-CH-Gruppen beobachtet man bei $\delta=6.25$ und bei $\delta=6.33$ als Dublett vom Triplett mit $J=15.5$ und $7.5 \mathrm{~Hz}$ entsprechend einer trans-Kopplung zu 3'/3''-H und einer Kopplung zu 1'/1''-H $\mathrm{H}_{2}$ 3'-H und 3' '-H wiederum resonieren bei $\delta=6.46$ und bei $\delta=6.48$ als Dublett mit $J=15.5 \mathrm{~Hz}$. Im Tieffeld sieht man das Multiplett-Signal der Phenyl-Protonen bei $\delta=7.15-7.42$.

Im ${ }^{13}$ C-NMR-Spektrum erkennt man bei $\delta=18.09$ das Signal der Methyl-Gruppe. Bei $\delta=34.63$ und $\delta=36.19$ findet sich die Resonanz von C-1' und C-1"' und die von C-2, C-1 und $\mathrm{C}-3$ bei $\delta=45.04, \delta=67.65$ und $\delta=73.62$. Die Doppelbindungs-Kohlenstoffe resonieren bei $\delta=125.8$ und $\delta=126.0$ für C-3' und C-3', sowie bei $\delta=127.1$ und $\delta=127.2$ für C-2' und C-2', Die aromatischen C-5', C-9', C-5' und C-9', geben Signale bei $\delta=126.0$, C-7' und C7' 'bei $\delta=128.5$ und C-6', C-8', C-6' ' und C-8' ' bei $\delta=133.1$. Die Resonanzen der quartären C-4' und C-4', erscheinen bei $\delta=137.3$.

Das IR-Spektrum zeigt bei $3372 \mathrm{~cm}^{-1}$ die Bande der OH-Gruppen und bei 3034 und $2970 \mathrm{~cm}^{-1}$ die C-H-Streckschwingungen. Bei $1651 \mathrm{~cm}^{-1}$ erkennt man die Bande der olefinischen Bindungen und bei 1448 und $1378 \mathrm{~cm}^{-1}$ die der $\mathrm{CH}_{3}$-Deformationsschwingung.

Das DCI-Massenspektrum zeigt die Massenpeaks M+18, M+18+17 und $2 \mathrm{M}+18$ bei $\mathrm{m} / \mathrm{z}=$ 338,355 und 658.

Im ${ }^{1}$ H-NMR-Spektrum der Verbindung 252 erkennt man bei $\delta=1.72$ ein breites SingulettSignal der beiden $\mathrm{OH}-G r u p p e n .1$ ' $-\mathrm{H}_{2}$ und 1'' $-\mathrm{H}_{2}$ resonieren bei $\delta=2.23$ als Dublett mit $J=7.1 \mathrm{~Hz}, 1-\mathrm{H}_{2}$ und 3- $\mathrm{H}_{2}$ bei $\delta=3.66$ als Singulett. Das Signal von 2'-H und 2' '-H beobachtet man als Dublett vom Triplett bei $\delta=5.97$ mit einer trans-Kopplung von $J=15.1 \mathrm{~Hz} z u$ 3'/3''-H und einer Kopplung von $J=7.1 \mathrm{~Hz}$ zu 1'/1' '- $\mathrm{H}_{2}$. 3'-H und 3''-H resonieren bei $\delta=6.33$ als Dublett mit $J=15.1 \mathrm{~Hz}$. Die aromatischen 8'-H und 8' '-H ergeben ein zentriertes Multiplett-Signal bei $\delta=6.52$, die übrigen Signale der Furan-Ringe sammeln sich als Multiplett bei $\delta=7.33-7.42$.

Im ${ }^{13} \mathrm{C}$-NMR-Spektrum erkennt man bei $\delta=35.40$ das Signal der Methylenkohlenstoffatome der Allyleinheiten. Das quartäre C-2 resoniert bei $\delta=43.15$ und C-1 bzw. C-3 bei $\delta=68.53$. 
Die Resonanz der aromatischen C-8'/C-8', beobachtet man bei $\delta=107.4$ und die der olefinischen Kohlenstoffatome C-3'/C-3' ' und C-2'/C-2', bei $\delta=121.4$ und 124.9. Das Signal der quartären C-4'/C-4'" erkennt man bei $\delta=124.1$. Die zum Sauerstoffatom des Furan-Ringes ortho-ständigen Kohlenstoffatome C-5'/C-5' und C-7'/C-7', resonieren bei $\delta=139.7$ und 143.4.

Das IR-Spektrum zeigt bei $3345 \mathrm{~cm}^{-1}$ die Bande der OH-Gruppen und bei $2926 \mathrm{~cm}^{-1}$ die C-HStreckschwingungen. Bei $1713 \mathrm{~cm}^{-1}$ erkennt man die Bande der olefinischen Bindungen.

Das DCI-Massenspektrum zeigt die Massenpeaks $M+18$ und $M+18+17$ bei $\mathrm{m} / \mathrm{z}=306$ und 323.

\subsection{Verwendung von Allylcarbonaten}

Neben dem Einsatz von Allylacetaten für allylische Alkylierungen bietet sich auch die Verwendung von Allylcarbonaten an, da hierbei keine Base zugesetzt werden muß, um die 1,3-Dicarbonylverbindung zu deprotonieren (siehe Kapitel 3.1.). Zudem verlaufen die Reaktionen bei niedrigeren Temperaturen aufgrund der höheren Reaktivität der Allylcarbonate gegenüber den Allylacetaten. Dies wurde am Beispiel der Umsetzung der Allylcarbonate 196 und 198 mit polymergebundenem Acetoacetat $\mathbf{1 3 8}$ bei Raumtemperatur innerhalb von 16 Stunden gezeigt. Nach reduktiver Abspaltung von der festen Phase mit DIBAH erhielt man die gewünschten dialkylierten Produkte 202 bzw. 239 in guten Gesamtausbeuten von 68 bzw. 75\%. Im Vergleich zu den Synthesen mit Allylacetaten konnte hier durch Verlängerung der Reaktionszeiten die Umsetzung bei Raumtemperatur durchgeführt werden, die Selektivität zugunsten der dialkylierten Produkte wurde davon nicht beeinflußt.
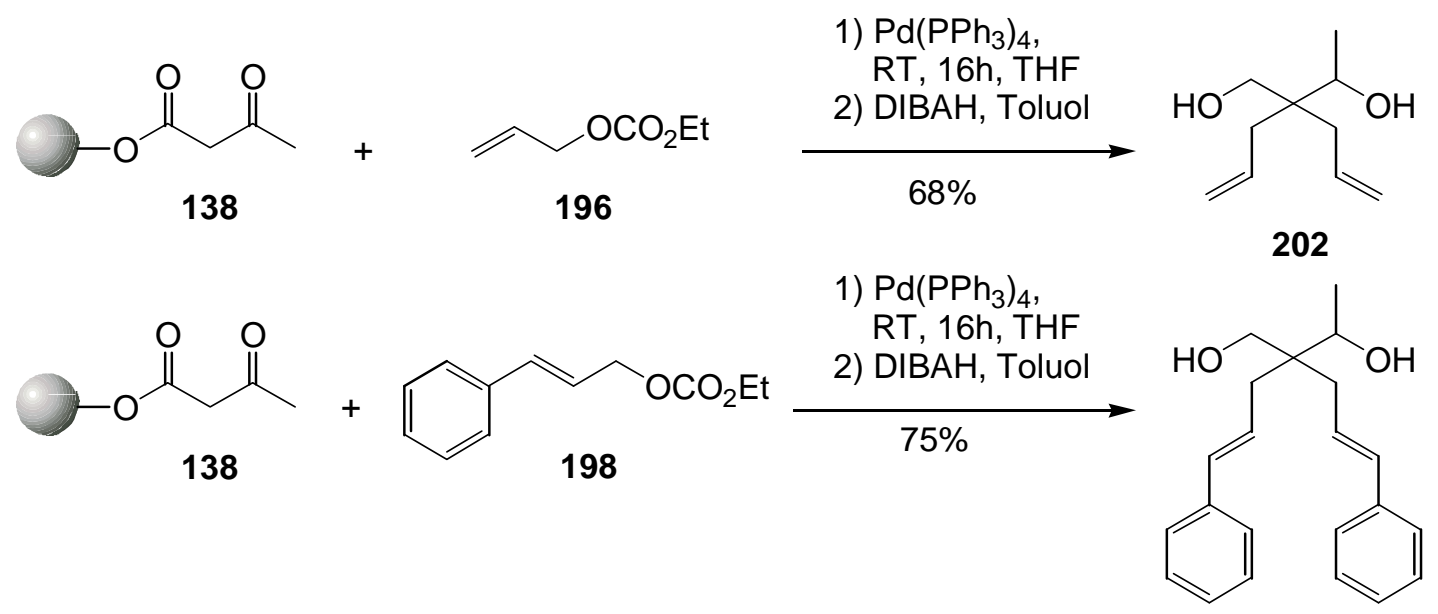

239

Weitere Synthese unter Verwendung von Allylcarbonaten werden in Kapitel 6.8.2. besprochen. 


\subsection{Verwendung von Allylchloriden}

Allylchloride stellen eine weitere Klasse von Substanzen dar, die für Palladium-katalysierte allylische Alkylierungen eingesetzt werden können. Ihre Verwendung ist jedoch durch den im Vergleich mit den Allylacetaten und -carbonaten erschwerten Zugang deutlich eingeschränkt.

Die Umsetzung des polymergebundenen Methylmalonats 143 mit dem kommerziell erhältlichen 3-Chlor-2-methyl-prop-1-en 255 gelang mit $n$-Butyllithium als Base. Hierzu wurde 143 zunächst bei $0{ }^{\circ} \mathrm{C}$ mit einem Äquivalent der Base versetzt und 15 Minuten bei Raumtemperatur gerührt. Anschließend gab man das Allylchlorid 255 und Tetrakis(triphenylphosphan)palladium hinzu und ließ 20 Stunden bei Raumtemperatur reagieren. Nach reduktiver Abspaltung von der festen Phase erhielt man ein Gemisch der mono- und dialkylierten Diole 257 und 212 in Ausbeuten von 28 und $10 \%$.

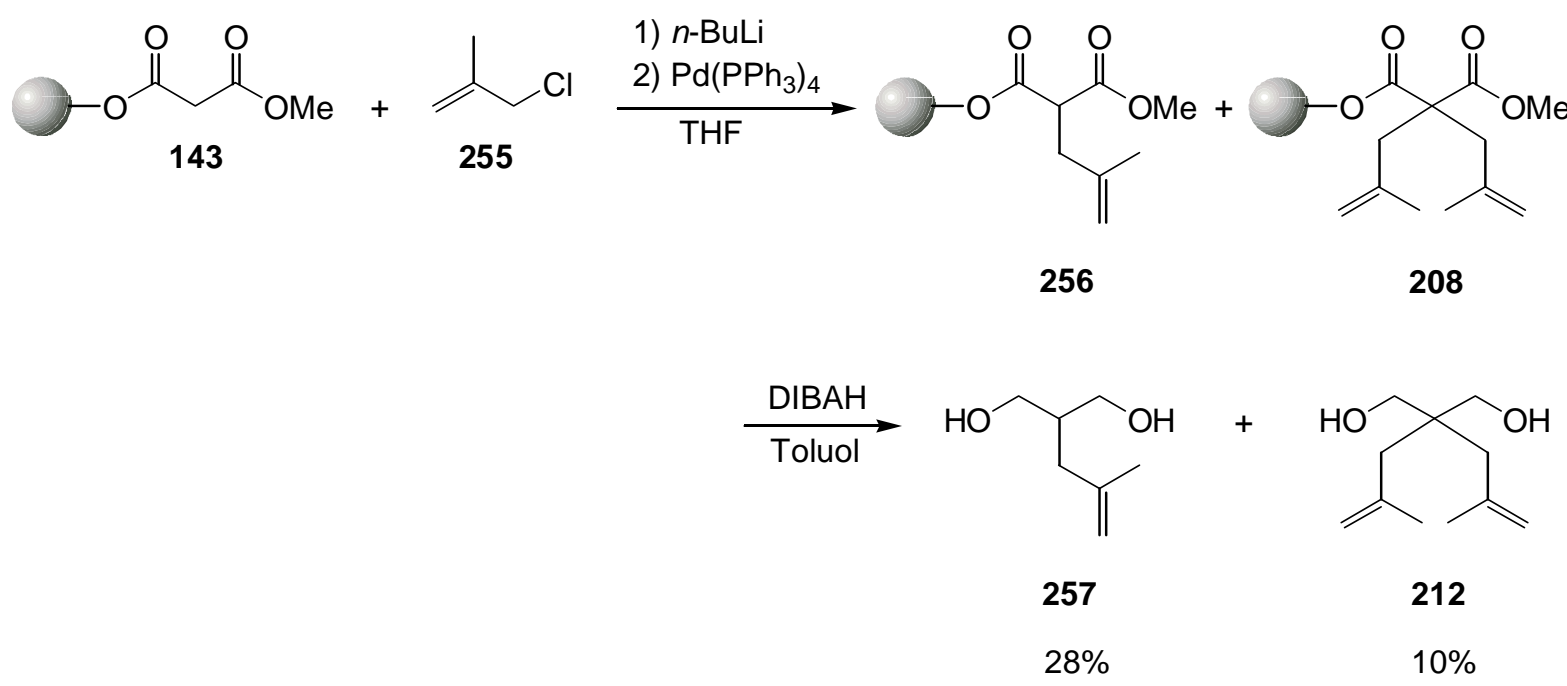

Obwohl nur eine Äquivalent Base verwendet wurde, beobachtete man auch die Bildung des dialkylierten Produktes 212. Dies könnte durch eine unvollständige Deprotonierung vor bzw. durch solvensvermittelte Deprotonierungs-Reprotonierungs-Prozesse zwischen deprotoniertem Methylmalonat 143 und monoalkyliertem Methylmalonat 256 während der Palladiumkatalysierten Reaktion erklärt werden. ${ }^{[14 a]}$

\subsection{Optimierungsversuche}

Die oben beschriebenen allylischen Alkylierungen an der festen Phase verlaufen in guten bis sehr guten Gesamtausbeuten. Lediglich die Umsetzungen mit den unreaktiveren heteroaroma- 
tisch-substituierten Allylacetaten ergeben die gewünschten Produkte nur in mäßigen Ausbeuten. Es ist bekannt, daß der intermediär gebildete $\pi$-Allyl-Palladium-Komplex $\mathbf{4 8}$ in Gegenwart von Phosphan-Liganden mit einem reaktiveren kationischen Komplex 49 im Gleichgewicht steht (siehe Kapitel 3.1.), welcher bei bidentalen Liganden entropisch begünstigt ist.

Durch Zugabe von 40 mol\% Triphenylphosphan zur Reaktion des Thiophen-substituierten Allylacetats 191 mit polymergebundenem Methylmalonat 138 war keine Steigerung der Ausbeute zu erzielen. Auch bidentale Liganden wie 1,2-Bis(diphenylphosphino)-ethan (DPPE) 258 führten nicht zu einer Verbesserung der Ausbeute. So erhielt man bei der Reaktion des Phenylsubstituierten Allylacetats $\mathbf{1 6 0}$ mit 138 ein Gemisch des mono- und dialkylierten Produkts im Verhältnis von $2: 1$ in 40\%iger Ausbeute. Bei dieser Umsetzung konnte ebenfalls gezeigt werden, daß ein Wechsel der Palladium-Quelle keinen positiven Effekt auf die Reaktion ausübt.

Auf die Verringerung der Katalysatormenge wurde bereits in Kapitel 6.1. eingegangen.<smiles>COC(=O)CC(=O)Oc1ccccc1</smiles>

143<smiles>C=CC(OC(C)=O)c1cccs1</smiles>

191<smiles>COC(=O)CC(=O)Oc1ccccc1</smiles>

143

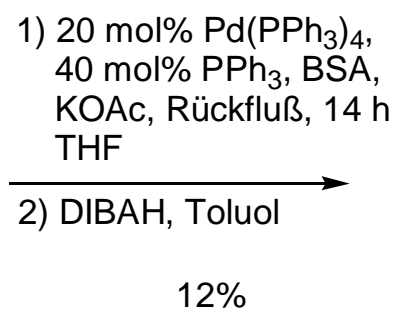

$12 \%$

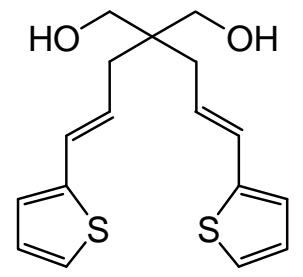

254

1) $10 \mathrm{~mol} \% \mathrm{Pd}_{2}(\mathrm{dba})_{3} \cdot \mathrm{CHCl}_{3}$, 20 mol\% DPPE, BSA, KOAc, Rückfluß, 6 h THF

2) DIBAH, Toluol

$40 \%$

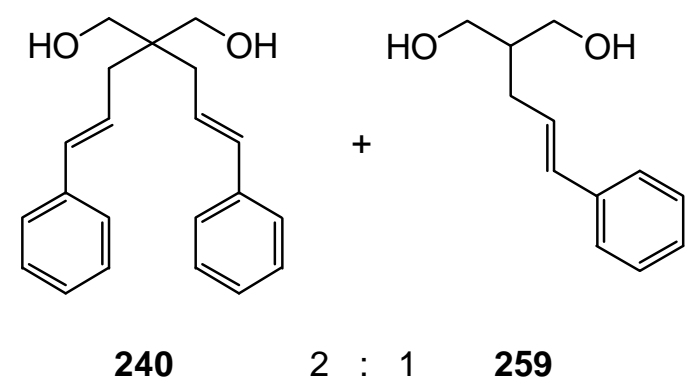

Ähnliche Ergebnisse waren bei der Variation des Lösungsmittels zu beobachten. Sowohl in Dimethylformamid als auch in Acetonitril verliefen die allylischen Alkylierungen in vergleichbaren Ausbeuten. Es konnte also gezeigt werden, daß die Reaktion durch Additive bzw. Wechsel des Lösungsmittels nicht verbessert werden konnte. 
Eine Variation der Reaktionstemperatur und -zeit hingegen zeigte deutliche Auswirkungen auf den Reaktionablauf. So konnte die Umsetzung von Allylacetat 199 mit polymergebundenem Acetoacetat 138 bei Raumtemperatur mit einer verlängerten Reaktionszeit von 10 Stunden durchgeführt werden. Nach Abspaltung von der festen Phase erhielt man 202 in einer hervorragenden Gesamtausbeute von 87\%, also im selben Bereich wie bei der Synthesedurchführung unter Rückflußbedingungen. Eine Übertragung auf die aromatischen Allylacetate gelang ebenfalls, wie die Alkylierung festphasengebundenen Acetoacetats 138 mit Phenylallylacetat 160 zeigte. Das gewünschte 1,3-Diol 239 wurde in 50\%iger Gesamtausbeute erhalten wurde, verglichen mit 52\% Ausbeute unter Rückflußbedingungen. Die Reaktionszeit mußte allerdings deutlich auf 24 Stunden verlängert werden, da bei kürzeren Reaktionszeiten Gemische aus mono- und dialkyliertem Produkt isoliert wurden.<smiles>CC(=O)CC(=O)Oc1ccccc1</smiles>

138

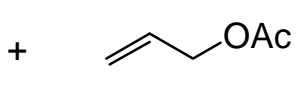

199
1) $\mathrm{Pd}\left(\mathrm{PPh}_{3}\right)_{4}$ BSA, KOAc, RT, $14 \mathrm{~h}, \mathrm{THF}$

2) DIBAH, Toluol

$87 \%$

1) $\mathrm{Pd}\left(\mathrm{PPh}_{3}\right)_{4}$, BSA, KOAc, RT, $24 \mathrm{~h}$, THF

2) DIBAH, Toluol $50 \%$<smiles>C=CCC(CO)(CC=C)C(C)O</smiles>

202<smiles>CC(=O)CC(=O)Oc1ccccc1</smiles>

138
160<smiles>CC(=O)OC/C=C/c1ccccc1</smiles> 


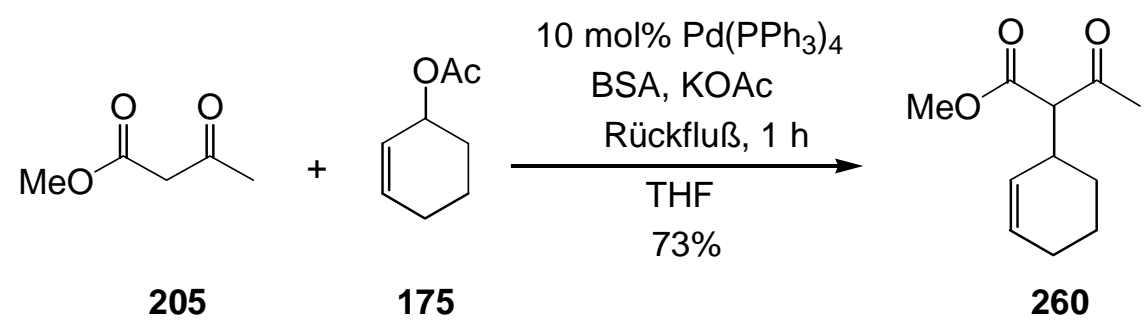

\subsection{Variation der Abspaltungsbedingungen}

Neben der Reduktion der Estergruppe zum Alkohol kann die Abspaltung von der festen Phase auch durch basische Umesterung ${ }^{[59]}$ erfolgen, wodurch man die entsprechenden Alkylester erhält. Hierzu wurde dialkyliertes Methylmalonat 201 für drei Stunden bei Raumtemperatur mit drei Äquivalenten Natriummethanolat 261 in Tetrahydrofuran umgesetzt. Nach Waschen des Harzes mit Methanol wurde das Filtrat mit saurem Ionentauscher (Amberlite IR 120) neutralisiert. Nach erneuter Filtration und Einengen im Vakuum erhielt man den Dimethylmalonsäureester 262 in einer Ausbeute von 41\%.

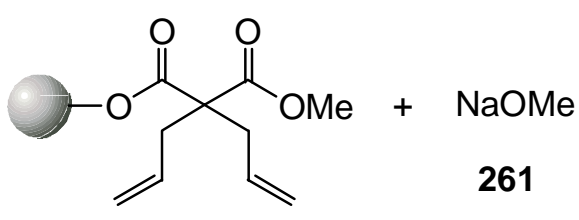

201

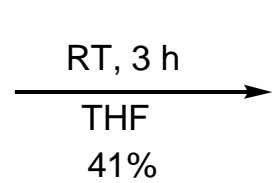<smiles>C=CCC(CC=C)(C(=O)OC)C(=O)OC</smiles>

262

Bei der Abspaltung des polymergebundenen Acetoacetats 233 mit Natriummethanolat erhielt man in einer Ausbeute von 53\% den Methylester 263, der sich infolge einer Retro-ClaisenKondensation aus dem primären Abspaltungsprodukt gebildet hat. Diese sog. Esterspaltung ${ }^{[63]}$ erfolgt, wie aus der Chemie in flüssiger Phase bekannt, bei $\alpha, \alpha$-dialkylierten 1,3Dicarbonylverbindungen besonders leicht, $\alpha$-monoalkylierte Acetoacetate hingegen sind unter den Reaktionsbedingungen stabil (siehe Kapitel 6.8.).<smiles>CC(=O)C(C/C=C/c1ccccc1)(C/C=C/c1ccccc1)C(=O)Oc1ccccc1</smiles>

$+\mathrm{NaOMe}$

261

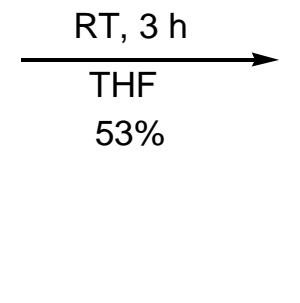<smiles>COC(=O)C(C/C=C/c1ccccc1)C/C=C/c1ccccc1</smiles>

263 
Die Abspaltung durch Umesterung muß unter Feuchtigkeitsausschluß durchgeführt werden, da sonst Gemische aus den gewünschten Methylestern und den entsprechenden Carbonsäuren gebildet werden. Letztere können auch gezielt synthetisiert werden, indem der Reaktionslösung Wasser zugesetzt wird. Auf diese Weise konnte 264 selektiv ausgehend von dem festphasengebundene Acetoacetat 200 in einer Ausbeute von 56\% erhalten werden.

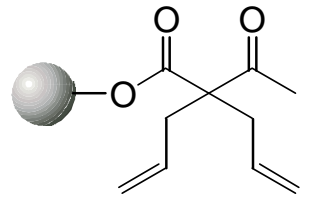

200
$+\mathrm{NaOMe}$

261

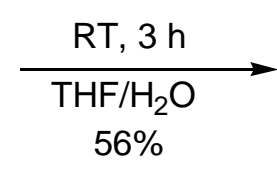

$56 \%$

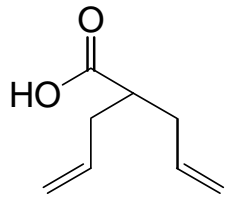

264

Eine weitere Möglichkeit der Abspaltung polymergebundener Ester besteht in der Umsetzung mit Aminen unter Ausbildung der entsprechenden Amide. So gelang es, polymergebundenes Methylmalonat 143 mit 20 Äquivalenten Benzylamin 265 innerhalb von 14 Stunden unter Rückflußbedingungen in THF in das Malonsäurediamid 266 umzuwandeln, welches aus der Waschlösung auskristallisierte.

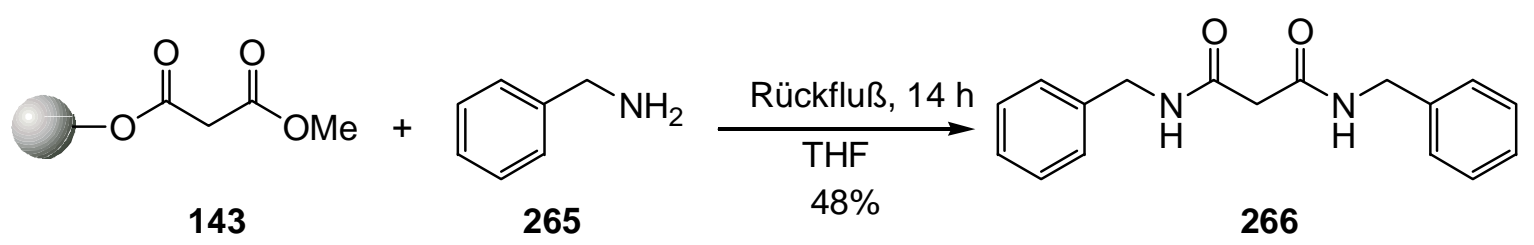

Durch die beschriebenen Abspaltungsmethoden kann die Diversität der Reaktionssequenz stark erweitert werden, da neben den 1,3-Diolen auch Ester und Carbonsäuren zugänglich sind. Ein weitere Vorteil besteht darin, daß diese Diversität im Abspaltungsschritt eingeführt werden kann, der oft durch den verwendeten Linker vorgegeben ist.

\subsubsection{Diskussion ausgewählter spektroskopischer Daten}

Im ${ }^{1} \mathrm{H}-\mathrm{NMR}-$ Spektrum der Verbindung 263 erkennt man die Signale von 2-H, 3- $\mathrm{H}_{2}$ und 1'- $\mathrm{H}_{2}$ als Multiplett bei $\delta=2.37-2.75$, während die Methyl-Gruppe des Esters bei $\delta=3.68$ als Singulett resoniert. Das 4-H- und 2'-H-Proton sind bei $\delta=6.15$ als Dublett vom Triplett mit $J=15.5$ und $6.9 \mathrm{~Hz}$ zu erkennen. Das Dublett bei $\delta=6.45$ mit einer transKopplungskonstanten von $J=15.5 \mathrm{~Hz}$ kann der Resonanz von 5-H und 3'-H zugeordnet werden. Im Tieffeld erkennt man schließlich das Multiplett-Signal der aromatischen Protonen bei $\delta=7.16-7.40$. 
Das ${ }^{13} \mathrm{C}-\mathrm{NMR}$-Spektrum zeigt bei $\delta=35.51$ die Resonanz der beiden Methylen-Gruppen und bei $\delta=45.80$ das Signal des C-2. Die Methyl-Gruppe des Esters resoniert bei $\delta=51.09$. Die ortho-ständigen aromatischen Kohlenstoffatome erzeugen Signal bei $\delta=126.5$, während die $m e t a$-ständigen bei $\delta=128.7$ resonieren. Die olefinischen Signale erscheinen bei $\delta=127.1$ für C-4 und C-2' sowie bei $\delta=132.7$ für C-5 und C-3'. Die quartären aromatischen Kohlenstoffatome resonieren bei $\delta=137.8$ und die Carbonyl-Gruppe bei $\delta=174.7$.

Das IR-Spektrum zeigt bei 3029 und $2953 \mathrm{~cm}^{-1}$ die Banden der C-H-Streckschwingungen und bei $1728 \mathrm{~cm}^{-1}$ die der Carbonylgruppe des Estern. Die Schwingung der olefinischen Bindungen erkennt man bei $1651 \mathrm{~cm}^{-1}$.

Das DCI-Massenspektrum zeigt die Massenpeaks $M+1, M+18$ und $M+18+17$ bei $\mathrm{m} / \mathrm{z}=307$, $324,341$.

\subsection{Verwendung von Bisallyltemplaten}

Die bisher beschriebenen Synthesen an der festen Phase waren dadurch gekennzeichnet, daß die $\pi$-Allyl-Palladium-Spezies in Lösung generiert und mit dem polymergebundenen Nucleophil umgesetzt wurde. Eine Reaktionsführung im umgekehrten Sinne wird ermöglicht durch Palladium-katalysierte Umsetzungen mit Bisallyltemplaten, also mit Verbindungen, die zwei Abgangsgruppen in Allylstellung besitzen. ${ }^{[40 a]}$ Nach Alkylierung des festphasengebundenen Nucleophils 138 kann in einer zweiten Reaktion durch Zusatz des Palladium-Katalysator eine polymergebundene $\pi$-Allyl-Palladium-Spezies aufgebaut werden, welche dann mit verschiedenen Nucleophilen umgesetzt werden kann. Dabei stehen eine Vielzahl unterschiedlicher Nucleophile zur Verfügung, so daß die Diversität der Reaktionssequenz stark erweitert werden kann. 


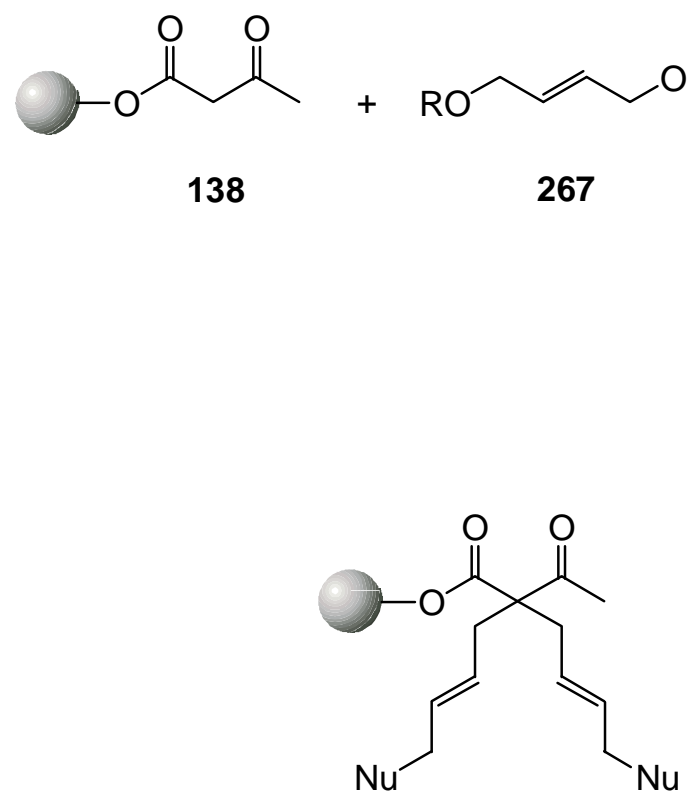

270
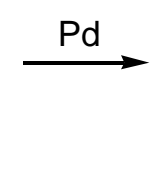<smiles>CC(=O)C(C/C=C/CO)(C/C=C/CO)C(=O)Oc1ccccc1</smiles><smiles>C1[Te]C[TeH]1</smiles><smiles>CC(=O)C(C/C=C\C#N)(C/C=C\C=[Pt])C(=O)Oc1ccccc1</smiles>

268

\subsubsection{Acetate als Bisallyltemplate}

Das Diacetat 272 ließ sich problemlos durch Acetylierung von (Z)-But-2-en-1,4-diol 271 mit zwei Äquivalenten Essigsäureanhydrid $\mathbf{1 5 5}$ in Gegenwart von Triethylamin und 4Dimethylaminopyridin in einer Ausbeute von $98 \%$ erhalten. Infolge des bereits erwähnten $\pi-\sigma-$ $\pi$-Mechanismus (siehe Kapitel 3.1.) ist bei allylischen Alkylierungen mit 272 von Produkten mit $E$-konfigurierten Doppelbindungen auszugehen.

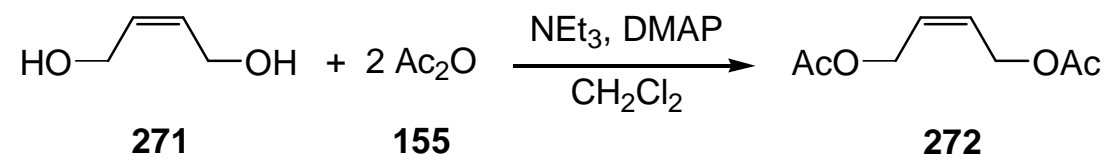

$98 \%$

Unter den erprobten Standardbedingungen wurde polymergebundenes Acetoacetat 138 mit dem Diacetat 272 zur Reaktion gebracht. Nach reduktiver Abspaltung von der festen Phase mit DIBAH erhielt man jedoch ein undefiniertes Gemisch von Produkten. Im ${ }^{1} \mathrm{H}-\mathrm{NMR}$-Spektrum waren keine Spuren der gewünschten Verbindung nachzuweisen. Auch Versuche, eine zweite Alkylierung vor der Abspaltung anzuschließen, schlugen fehl, sowohl bei der Reaktionsführung im Sinne einer Eintopf-Reaktion, bei der Methylacetoacetat 205 als Nucleophil nach einer Reaktionszeit von 5 Stunden zu der Mischung gegeben wurde, als auch bei getrennter Reaktionsfolge, bei der nach Waschen des Harzes nach 5 Stunden die Durchführung der zweiten Alkylierung in einem gesonderten Reaktionsschritt erfolgte. 
<smiles>CC(=O)CC(=O)Oc1ccccc1</smiles>

138<smiles>CC(=O)OC/C=C\COC(C)=[Fe]</smiles>

272
1) $\mathrm{Pd}\left(\mathrm{PPh}_{3}\right)_{4}, \mathrm{BSA}$

KOAc, THF

2) DIBAH, Toluol<smiles>CC(=O)CC(=O)Oc1ccccc1</smiles>

138<smiles></smiles>

272
1) $\mathrm{Pd}\left(\mathrm{PPh}_{3}\right)_{4}, \mathrm{BSA}$ KOAc, THF

2) 10 eq. Methylacetoacetat 205, $\mathrm{Pd}\left(\mathrm{PPh}_{3}\right)_{4}, \mathrm{BSA}, \mathrm{KOAc}, \mathrm{THF}$

3) DIBAH, Toluol

Ähnlich negative Ergebnisse lieferten die Synthesen ausgehend von dem monoacetylierten Butendiol 273, das durch Acetylierung von 271 mit nur einem Äquivalent Essigsäureanhydrid 155 in einer Ausbeute von $45 \%$ erhalten wurde. In diesem Fall soll zunächst selektiv die AcetatEinheit abreagieren. Nach Überführung des polymergebundenen Alkohols in das entsprechende Acetat kann dann die zweite Alkylierungsreaktion angeschlossen werden. Durch diese schrittweise Syntheseführung sollten weniger Nebenreaktionen auftreten. Aber sowohl die reduktive Abspaltung des erwünschten polymergebundenen Allylalkohols 274 als auch dessen Acetylierung an der festen Phase und anschließende palladium-katalysierte Umsetzung mit Methylacetoacetat 205 ergaben nur undefinierte Produktgemische.

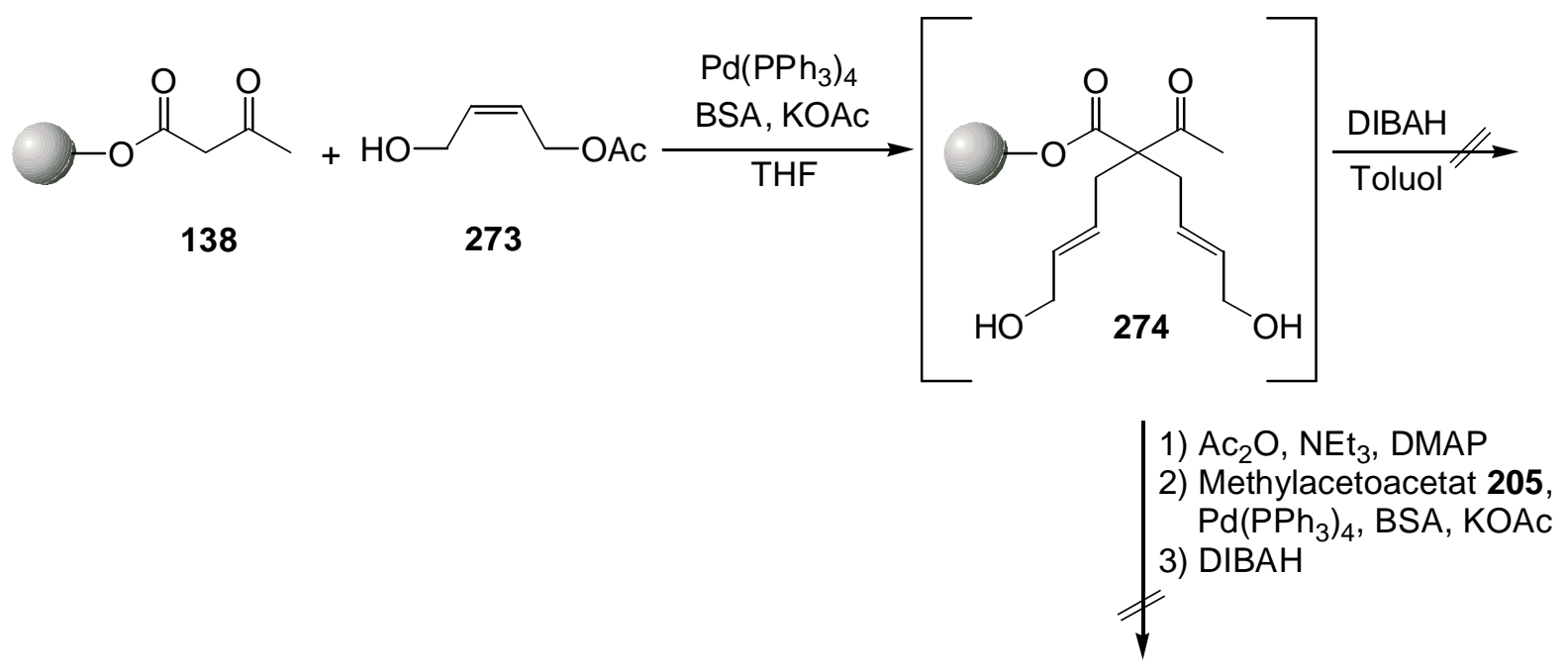

Da auch die Reaktion von 273 mit Methylacetoacetat 205 in flüssiger Phase zu einem Produktgemisch führte, wurden keine weiteren Umsetzungen mit den Acetaten 273 und 272 vorgenommen und statt dessen die entsprechenden Carbonate eingesetzt, mit denen die Reaktion bei milderen Bedingungen durchgeführt werden kann. 


\subsubsection{Carbonate als Bisallyltemplate}

Das Carbonat 275 und das Dicarbonat 276 konnten durch Umsetzung des (Z)-But-2-en-1,4diols 279 mit einem Äquivalent Chlorameisensäureethylester 195 durch langsames Zutropfen bei $0{ }^{\circ} \mathrm{C}$ in Ausbeuten von 64 bzw. $11 \%$ synthetisiert werden.
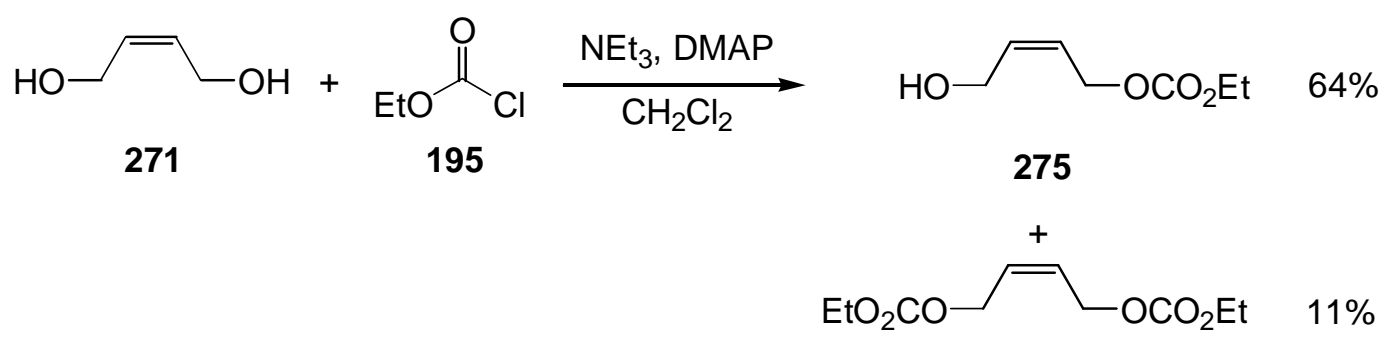

276

Nach den Mißerfolgen bei der Verwendung von Acetaten als Bisallyltemplate wurde zunächst die Eignung von Carbonaten für diesen Zweck getestet, indem Methylacetoacetat 205 mit zwei Äquivalenten des Hydroxycarbonats 275 in flüssiger Phase bei Raumtemperatur für eine Stunde umgesetzt wurde. Man erhielt die mono- und dialkylierten 1,3-Dicarbonylverbindungen 277 und 278 in Ausbeuten von 49 bzw. 40\%.

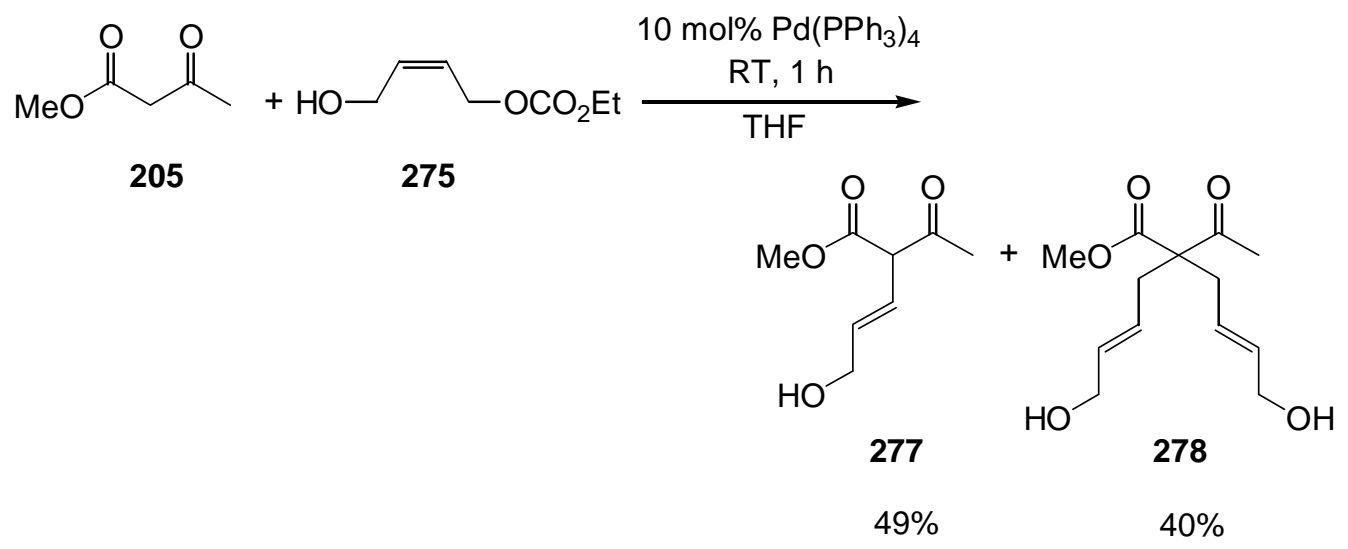

Die Übertragung an die feste Phase gelang problemlos. Durch Verwendung eines Überschusses an Carbonat 275 und die Verlängerung der Reaktionszeit auf 15 Stunden konnte selektiv die dialkylierte Verbindung 274 aufgebaut werden. Nach reduktiver Abspaltung vom polymeren Träger wurde das erhaltene Tetraol 279 direkt acetyliert. Man erhielt das Tetraacetat 280 ausgehend vom spacermodifizierten Merrifield-Harz 6 in einer Gesamtausbeute von 30\% über 4 Stufen. Dies zeigt, daß sich mit dem Hydroxycarbonat 275 polymergebundene Allylalkohole synthetisieren lassen, die nach Acetylierung zu den entsprechenden Allylacetaten Substrate für weitere palladium-katalysierte allylische Alkylierungen darstellen. 


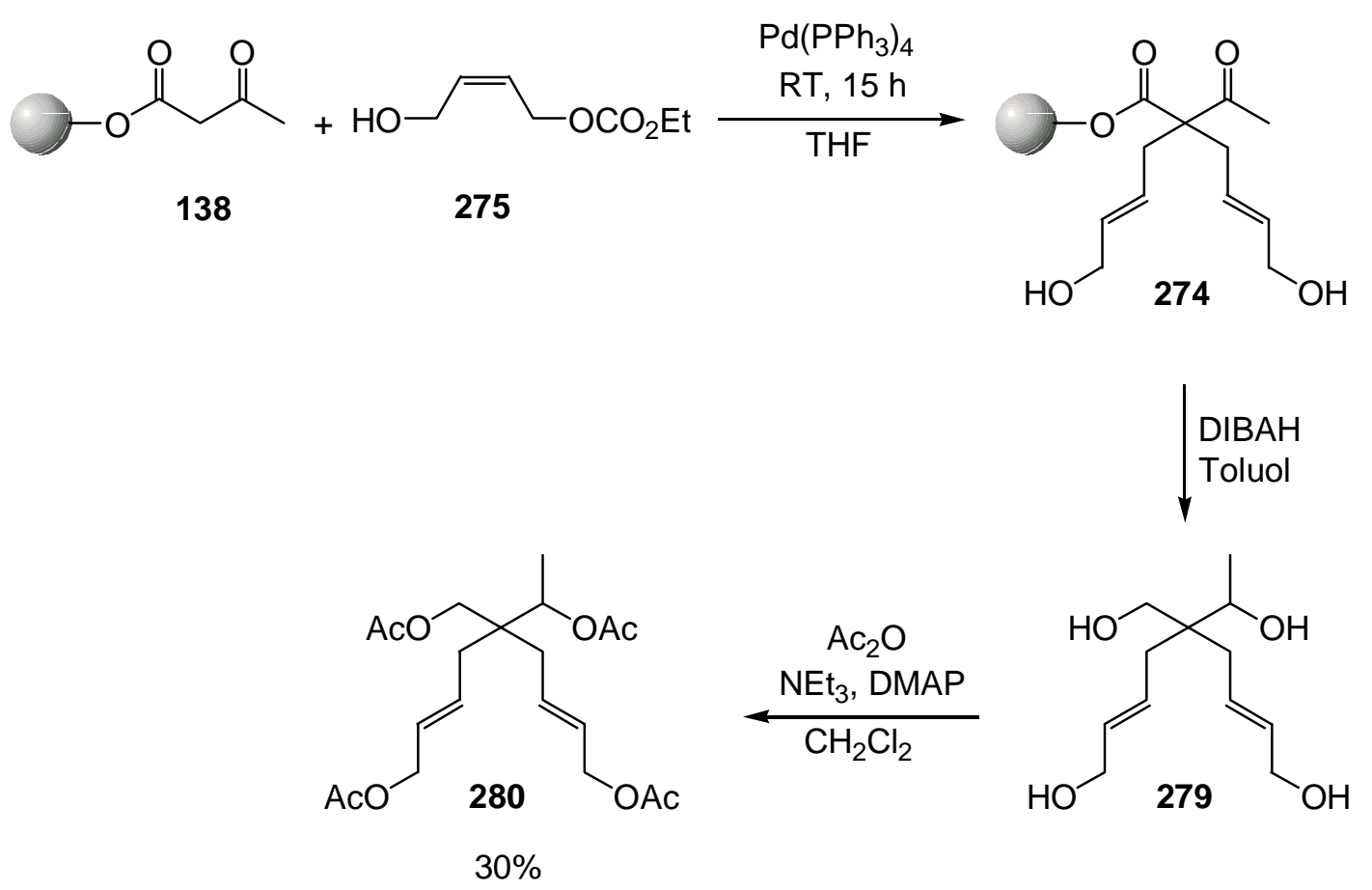

Zu diesem Zweck wurde 274 mit Essigsäureanhydrid in das Acetat 281 überführt. Charakteristisch für das Acetat 281 ist die IR-Bande der Acetat-Carbonyl-Gruppen bei $1738 \mathrm{~cm}^{-1}$. Die Umsetzung mit 20 Äquivalenten Methylacetoacetat 205 erfolgte unter den Standardbedingungen. Nach Abspaltung durch basische Umesterung mit Natriummethanolat von der festen Phase erhielt man das gewünschte Produkt 285 in einer Ausbeute von 50\%. Die analoge Reaktionssequenz mit Dimethylmalonat 282 als Nucleophil lieferte den Ester 286 in $40 \%$ iger Gesamtausbeute. 


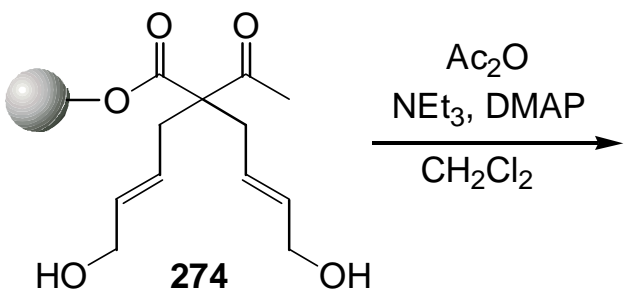<smiles>CC(=O)OC/C=C/CC(C/C=C/COC(C)=O)(C(C)=O)C(=O)Oc1ccccc1</smiles>
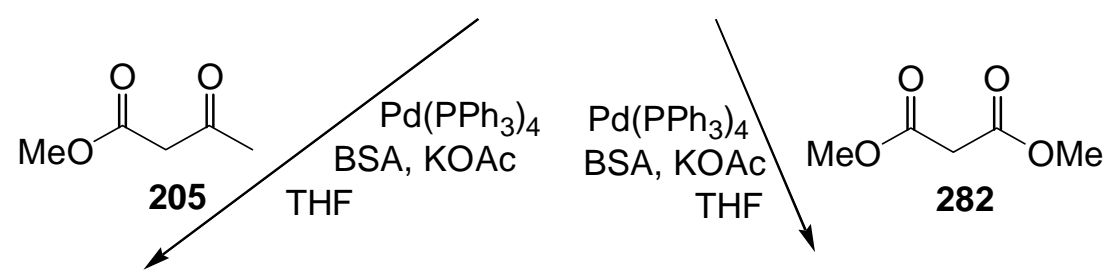<smiles>COC(=O)C(C/C=C/CC(C/C=C/CC(C(C)=O)C(C)=O)(C(C)=O)C(=O)Oc1ccccc1)C(C)=O</smiles>

283<smiles>COC(=O)C1C/C=C/CC(C/C=C/CC(C(C)=O)C(C)=O)C(C(C)=O)C(=O)C1</smiles>

285

$50 \%$<smiles>COC(=O)C(C/C=C/CC(C/C=C/CC(C(=O)OC)C(=O)OC)(C(C)=O)C(=O)Oc1ccccc1)C(C)=O</smiles>

284<smiles>COC(=O)C(C/C=C/CC(C(=O)OC)C(=O)OC)C/C=C/CC(C(=O)OC)C(=O)OC</smiles>

286

$40 \%$

In den oben durchgeführten Synthesen wurden große Überschüsse des Nucleophils eingesetzt, um sicherzustellen, daß die beiden polymergebundenen Allyleinheiten jeweils mit einer 1,3Dicarbonylverbindungen reagieren. Die Umsetzung mit nur einem Äquivalent Nucleophil sollte zu dessen doppelter Alkylierung unter Ausbildung eines 10-gliedrigen Ringes 287 führen. Dies konnte jedoch an der festen Phase nicht verwirklicht werden, da die Konzentration des Nucleophils infolge von Verdünnungseffekten (siehe Kapitel 2.2.) in diesem Fall zu gering war. Es wurde lediglich der Allylalkohol 288 und Spuren alkylierter Verbindungen erhalten. 


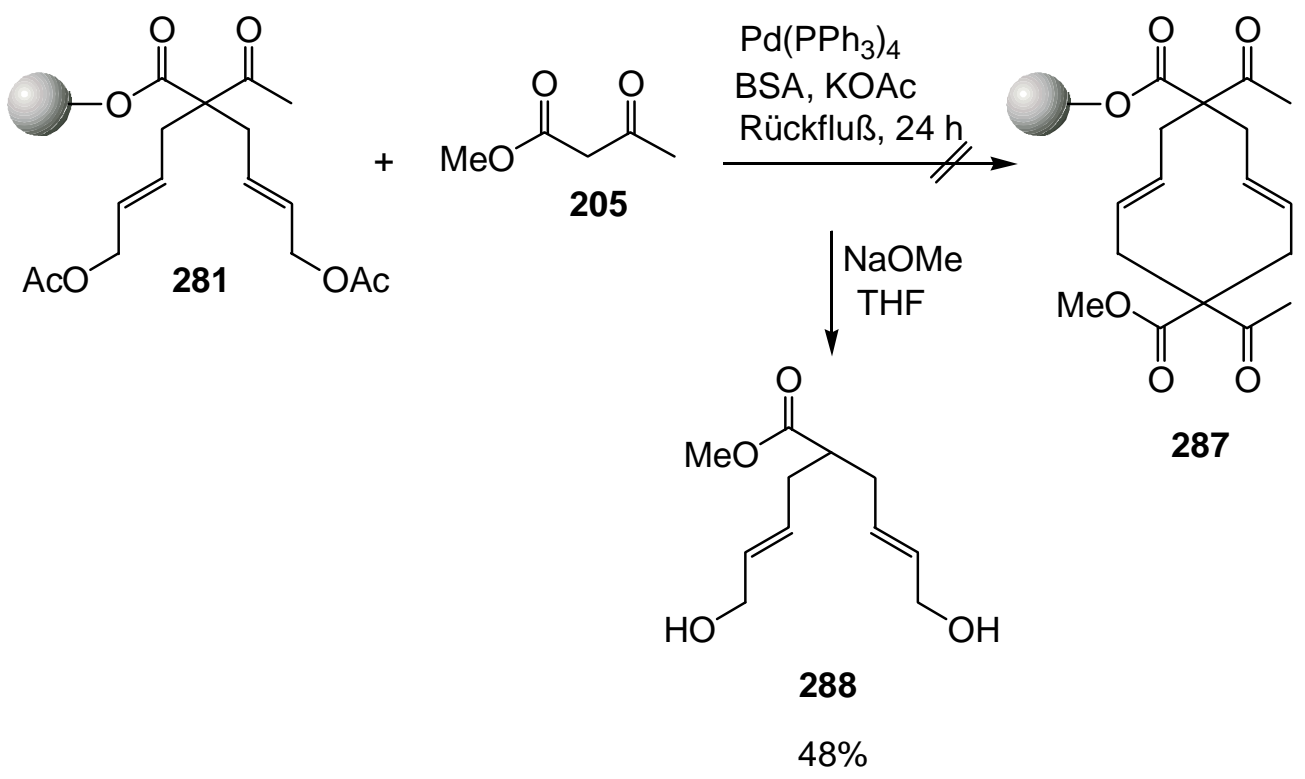

Die beschriebene Synthesesequenz an der festen Phase kann auch im Sinne einer EintopfReaktion durchgeführt werden. Hierzu wurde polymergebundenes Acetoacetat 138 mit dem Dicarbonat 276 umgesetzt, nach 5 Stunden bei Raumtemperatur das Nucleophil 205 in einem Überschuß von 20 Äquivalenten hinzugefügt und für weitere 10 Stunden gerührt. Nach basischer Abspaltung von der festen Phase erhielt man das gewünschte Produkt in einer Ausbeute von $45 \%$.

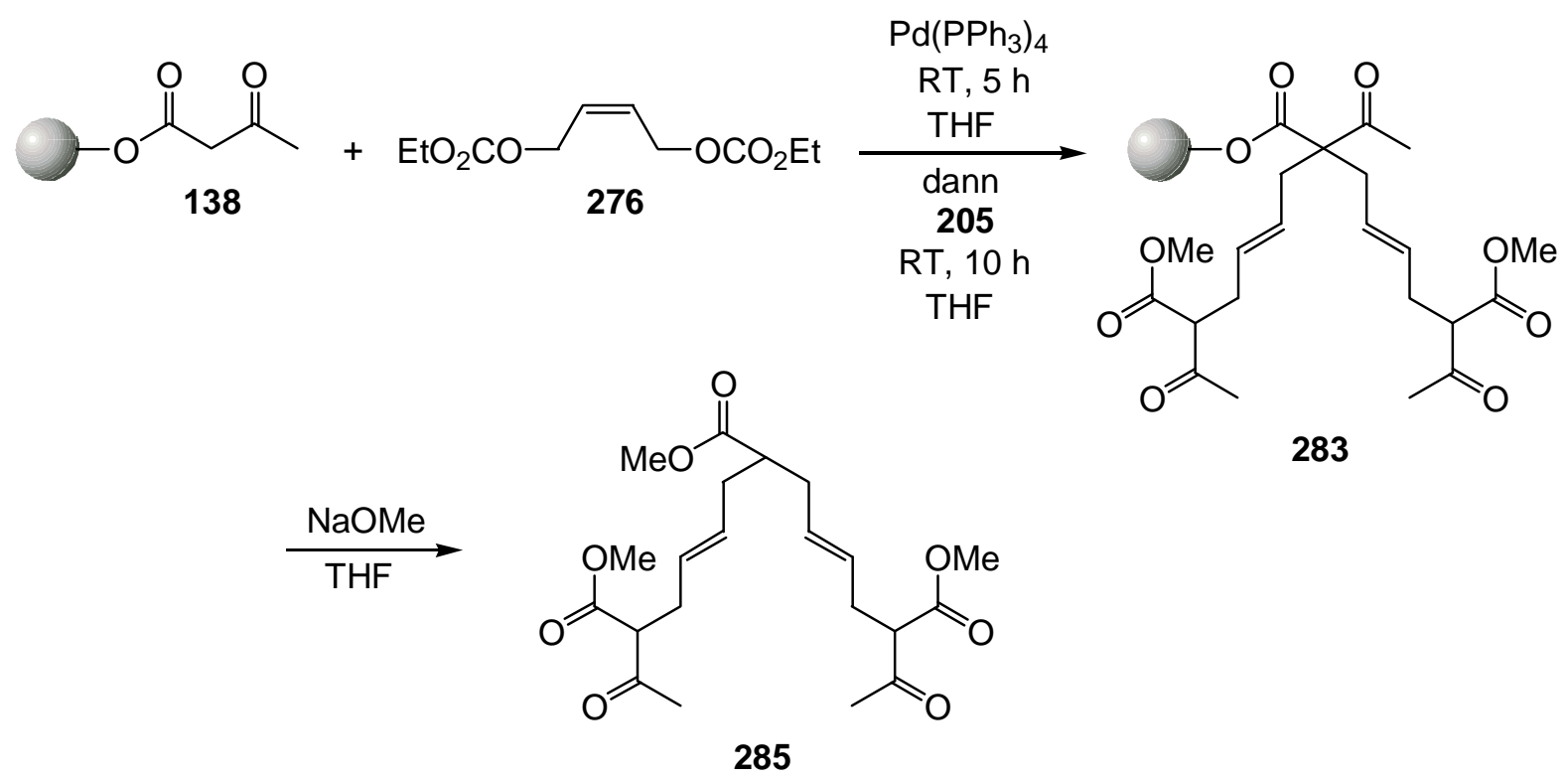

$45 \%$

Es zeigt sich, daß allylische Alkylierungen sowohl mit polymergebundenen Nucleophilen als auch mit festphasengebundenen $\pi$-Allyl-Palladium-Spezies möglich sind, wobei in letzteren Fall 
besonders die Möglichkeit der Eintopf-Reaktion eine effektive Methode zur C-C-Verknüpfung an der festen Phase darstellt.

\subsection{Synthesen am Wang-Harz}

Bei den bisher durchgeführten allylischen Alkylierungen wurde stets das spacermodifizierte Merrifield-Harz 6 als polymerer Träger verwendet. Ein weiteres Harz mit einer freien $\mathrm{OH}-$ Funktionalität ist das Wang-Harz 3 (siehe Kapitel 2.2.). Die Abspaltung polymergebundener Produkte erfolgt durch Umsetzung mit Trifluoressigsäure, die nach beendeter Reaktion leicht im Vakuum entfernt werden kann. Bei der Abspaltung von polymergebundenen Acetoacetaten erhält man die entsprechenden $\beta$-Ketosäuren, die spontan zu den Ketonen decarboxylieren. ${ }^{[64]}$ Auf diese Weise wird bei der Abspaltung die funktionelle Gruppe, die zur Anknüpfung an die feste Phase diente, durch ein Wasserstoff-Atom ersetzt. Somit handelt es sich hier um einen „traceless“ Linker, der jedoch nicht erst aufwendig synthetisiert werden muß wie andere Beispiele in Kapitel 2.2. zeigen. Ferner kann durch die Verwendung des Wang-Harzes 3 die Diversität der abgespaltenen Produkte weiter erhöht werden, da nun neben 1,3-Diolen, Estern und Carbonsäuren auch die entsprechenden Ketone zugänglich sind.

Die Synthese des polymergebundenen Acetoacetats 289 gelang analog zur Synthese von 138 durch Erhitzen des Wang-Harzes 3 mit 10 Äquivalenten Acetessigsäure-t-butylester 135 für drei Stunden am Rückfluß (siehe Kapitel 5.1.).

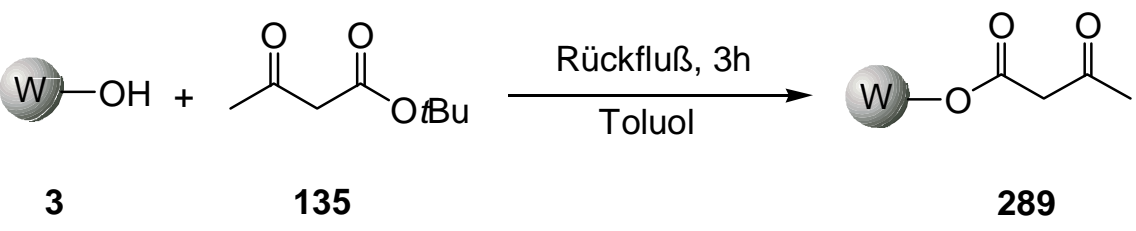

Um die Durchführbarkeit der allylischen Alkylierung von 289 zu demonstrieren, wurde 289 mit Allylacetat 199 und dem Phenyl-substituierten Allylcarbonat 198 umgesetzt. Nach säurekatalysierter Abspaltung vom Wang-Harz erhielt man die entsprechenden dialkylierten Ketone 290 und 291 in Gesamtausbeuten von 72 bzw. 60\% bezogen auf die Beladungsdichte des WangHarzes 3 von 1 mmol Hydroxy-Gruppen pro Gramm Harz. Man erhält also ähnliche Ausbeuten wie bei vergleichbaren Synthesen am spacermodifizierten Merrifield-Harz 6. 
<smiles>[CH2+]C(=O)CC(=O)Oc1ccccc1</smiles>

289<smiles>C=CCOC(C)=O</smiles>

199 \begin{tabular}{l} 
1) $\mathrm{Pd}\left(\mathrm{PPh}_{3}\right)_{4}$ \\
$\mathrm{BSA}, \mathrm{KOAc}$ \\
$\mathrm{RT}, 10 \mathrm{~h}, \mathrm{THF}$ \\
\hline 2) $\mathrm{RT}, 1 \mathrm{~h}$ \\
$20 \% \mathrm{TFA}$ \\
$\mathrm{CH}_{2} \mathrm{Cl}_{2}$
\end{tabular}

$72 \%$<smiles>CCOC(=O)OC/C=C/c1ccccc1</smiles>

1) $\mathrm{Pd}\left(\mathrm{PPh}_{3}\right)_{4}$ RT, 5h, THF

2) RT, $1 \mathrm{~h}$ $20 \%$ TFA $\mathrm{CH}_{2} \mathrm{Cl}_{2}$ $60 \%$<smiles>CC(=O)C(C/C=C/c1ccccc1)C/C=C/c1ccccc1</smiles>

Auch die Reaktion von Bisallyltemplaten gestaltete sich problemlos. Ausgehend von polymergebundenem Acetoacetat 289 konnte das Keton 295 in einer vierstufigen Synthesesequenz bestehend aus Alkylierung mit dem Allylcarbonat 275, Acetylierung des Allylalkohols 292, Umsetzung mit Methylacetoacetat 205 und saurer Abspaltung von der festen Phase in einer hervorragenden Gesamtausbeute von $66 \%$ erhalten werden.<smiles>CC(=O)C(C/C=C/CO)(C/C=C/CO)C(=O)Oc1ccc(C(F)(F)F)cc1</smiles>

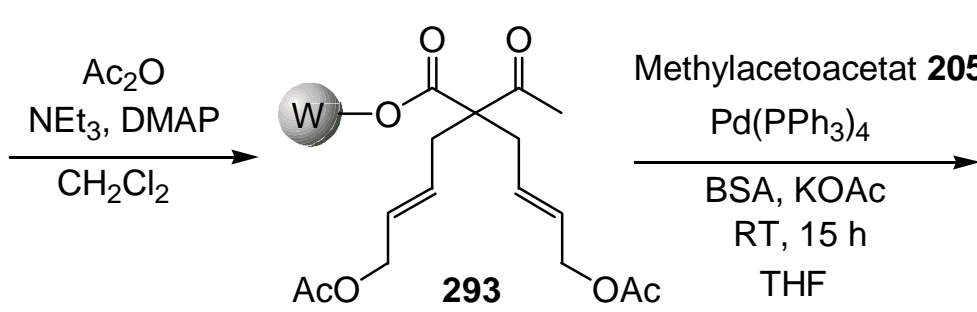

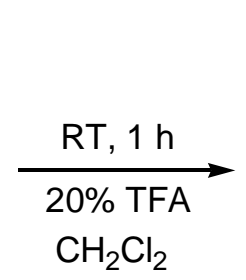<smiles>COC(=O)C(C/C=C/CC(C/C=C/CC(C(C)=O)C(C)=O)C(C)=O)C(C)=O</smiles>

294 


\section{Heck-Reaktionen an der festen Phase}

Eine retrosynthetische Betrachtung der allylischen Alkylierungen mit aromatischen Allylacetaten zeigt, daß sich die erhaltenen polymergebundenen Produkte auch in einer zweistufigen Synthesesequenz aufbauen lassen. Hierbei können die komplexen Allylacetate 297, die zunächst aus geeigneten Vorläufern synthetisiert werden müssen, zerlegt werden in Allylacetat 199 und Arylhalogenide, welche kommerziell in großem Umfang verfügbar sind. Nach der Generierung der Bisallyl-1,3-dicarbonylverbindung 298 an der festen Phase durch allylischen Alkylierung wird mittels einer Heck-Reaktion die gewünschte Verbindung 296 erhalten. Durch diese zweistufige Reaktionsführung kann die Flexibilität der Reaktion und die Diversität der Zielprodukte deutlich gesteigert werden.

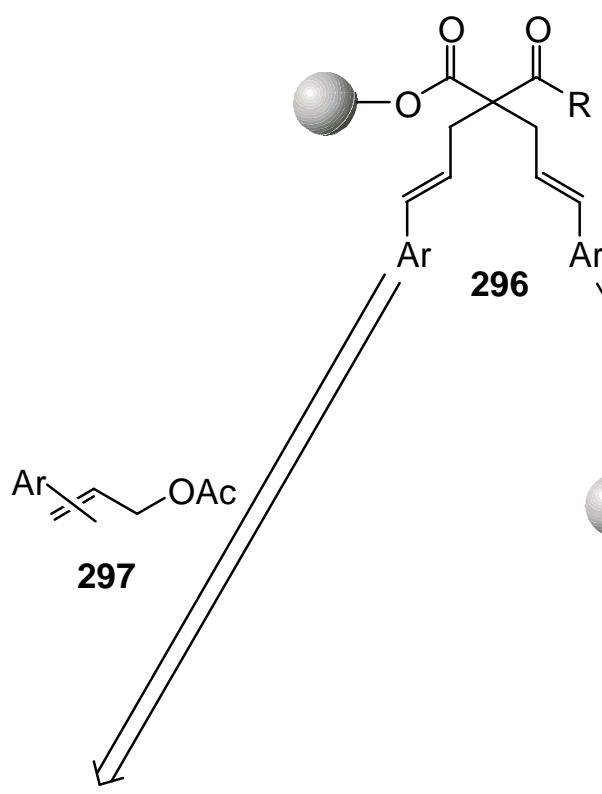<smiles>[R]C(=O)CC(=O)Oc1ccccc1</smiles>

138,143

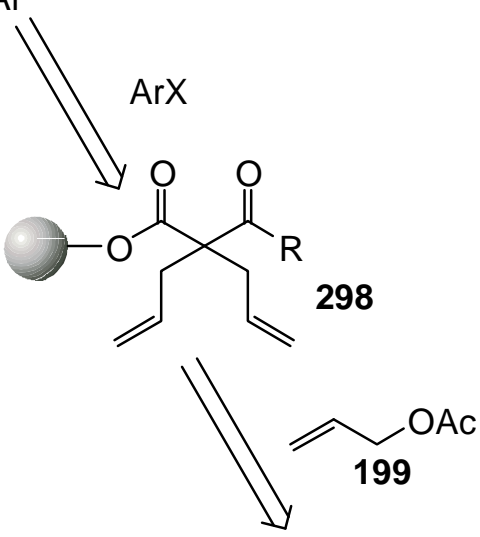<smiles>[R]C(=O)CC(=O)Oc1ccccc1</smiles>

138,143

\subsection{Verwendung von Iodaromaten}

Die Reaktionsbedingungen der durchgeführten Synthesen sind an Standardbedingungen für Heck-Reaktionen in Lösung angelehnt. So wurde polymergebundenes, dialkyliertes Acetoacetat 200 und Methylmalonat 201 mit Iodbenzol 299 bzw. 3-Iodanisol 300 unter Palladium- 
acetat-Katalyse zu den entsprechenden Heck-Produkten 233, 238 und 301 umgesetzt, die nach reduktiver Abspaltung von der festen Phase die 1,3-Diole 239, 243 und 302 in guten Gesamtausbeuten von 43 - 64\% lieferten (Tabelle 8). Dabei wurde in dieser Zwei-Stufen-Strategie das Anisol-Derivat $\mathbf{2 4 3}$ in einer Ausbeute von 53\% über vier Stufen erhalten, während die EinStufen-Strategie unter Verwendung des aromatischen Allylacetats 180 (siehe Kapitel 6.3.) die Zielverbindung lediglich in 23\%iger Gesamtausbeute über drei Stufen ergab.
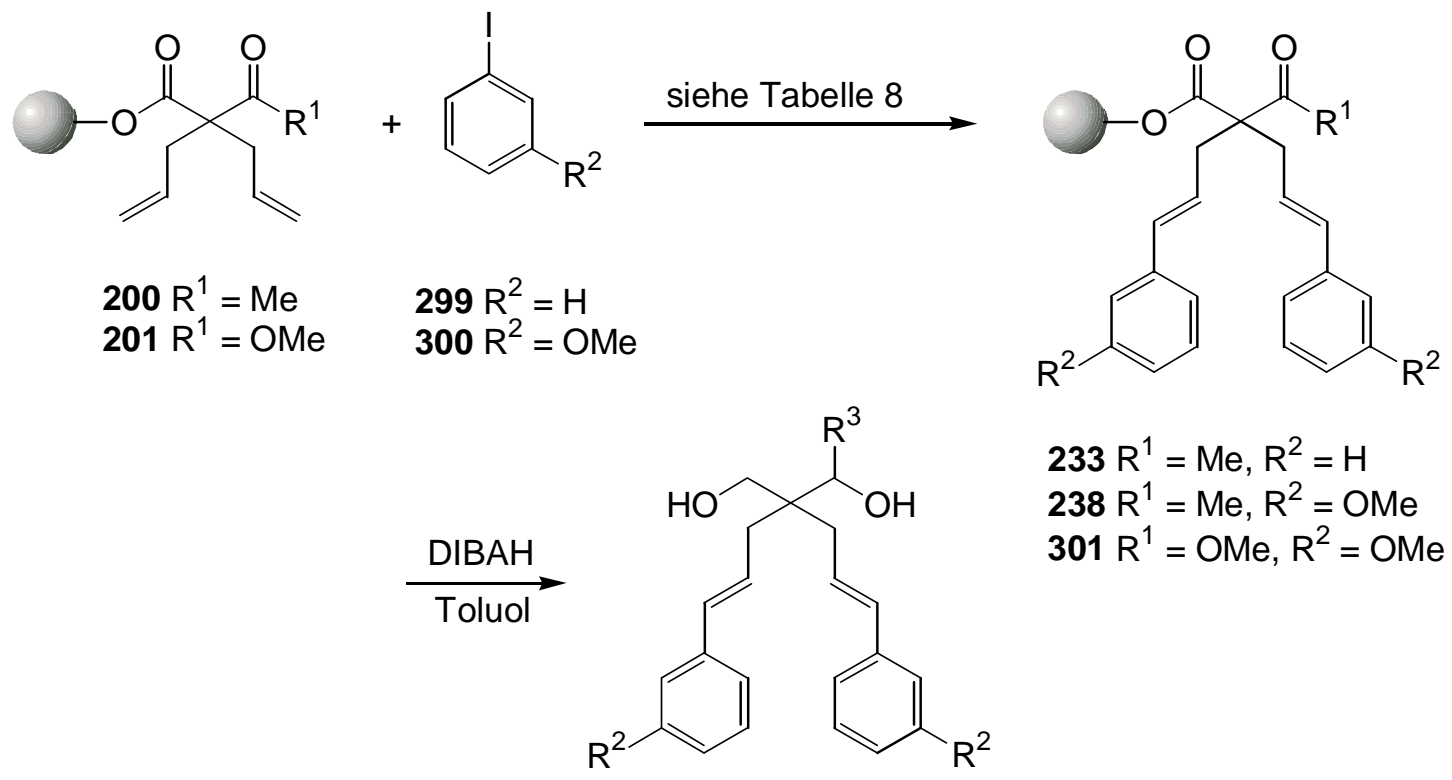

$\begin{array}{ll}239 R^{2}=H, R^{3}=M e & 64 \% \\ 243 R^{2}=O M e, R^{3}=M e & 53 \% \\ 302 R^{2}=O M e, R^{3}=H & 43 \%\end{array}$

\begin{tabular}{|c|c|c|c|c|c|c|c|}
\hline Edukt & Iodid & Katalysator & Zusätze & LM & Bedingungen & Produkt & $\begin{array}{c}\text { Ausbeute } \\
(\%)\end{array}$ \\
\hline $\mathbf{2 0 0}$ & $\mathbf{2 9 9}$ & $\begin{array}{c}20 \mathrm{~mol} \% \\
\mathrm{Pd}(\mathrm{OAc})_{2}\end{array}$ & 3 eq. $\mathrm{NEt}_{3}$ & $\mathrm{DMF}$ & $60{ }^{\circ} \mathrm{C}, 20 \mathrm{~h}$ & $\mathbf{2 3 9}$ & 15 \\
\hline $\mathbf{2 0 0}$ & $\mathbf{2 9 9}$ & $\begin{array}{c}20 \mathrm{~mol} \% \\
\mathrm{Pd}(\mathrm{OAc})_{2}\end{array}$ & 3 eq. $\mathrm{NEt}_{3}$ & $\mathrm{MeCN}$ & $80{ }^{\circ} \mathrm{C}, 7 \mathrm{~h}$ & $\mathbf{2 3 9}$ & 64 \\
\hline $\mathbf{2 0 0}$ & $\mathbf{3 0 0}$ & $\begin{array}{c}20 \mathrm{~mol} \% \\
\mathrm{Pd}(\mathrm{OAc})_{2}\end{array}$ & $\begin{array}{c}3 \text { eq. } \mathrm{KOAc}_{2} \\
1 \text { eq. } \mathrm{Bu}_{4} \mathrm{NCl}\end{array}$ & $\mathrm{DMF}$ & $100{ }^{\circ} \mathrm{C}, 21 \mathrm{~h}$ & $\mathbf{2 4 3}$ & 39 \\
\hline $\mathbf{2 0 0}$ & $\mathbf{3 0 0}$ & $\begin{array}{c}20 \mathrm{~mol} \% \\
\mathrm{Pd}(\mathrm{OAc})_{2}\end{array}$ & 3 eq. $\mathrm{NEt}_{3}$ & $\mathrm{THF}$ & $65{ }^{\circ} \mathrm{C}, 5 \mathrm{~h}$ & $\mathbf{2 4 3}$ & 53 \\
\hline $\mathbf{2 0 1}$ & $\mathbf{3 0 0}$ & $\begin{array}{c}20 \mathrm{~mol} \% \\
\mathrm{Pd}(\mathrm{OAc})_{2}\end{array}$ & 3 eq. $\mathrm{NEt}_{3}$ & $\mathrm{MeCN}$ & $80{ }^{\circ} \mathrm{C}, 7 \mathrm{~h}$ & $\mathbf{3 0 2}$ & 43 \\
\hline
\end{tabular}

Tabelle 8: Heck-Reaktionen mit Aryliodiden. 


\subsection{Verwendung von Bromaromaten}

Neben den oben beschriebenen Iodaromaten konnten auch die weniger reaktiven, aber im gröBeren Umfang kommerziell erhältlichen Bromaromaten in Heck-Reaktionen an der festen Phase eingesetzt werden. Ausgehend von dem dialkylierten Acetoacetat 200 und Methylmalonat 201 wurden mit Brombenzol 303 und 3-Bromanisol 304 nach Abspaltung vom polymeren Träger die 1,3-Diole 239, 240 und 243 in guten Gesamtausbeuten von 55 - 60\% erhalten (Tabelle 9). Im Vergleich zu den Umsetzungen mit den Iodaromaten mußten höhere Temperaturen und längere Reaktionszeiten verwendet werden, um analoge Ausbeuten zu erreichen. Die besten Ergebnisse wurden mit Palladiumacetat als Katalysator, Triphenylphosphan als Ligand und Kaliumacetat als Base unter Phasentransfer-Bedingungen in DMF erzielt. Auch mit Tributylamin als Base wurden gute Resultate erreicht, die Verwendung des Hermann-BellerKatalysators 89 jedoch ergab keinen positiven Effekt auf die Ausbeute.

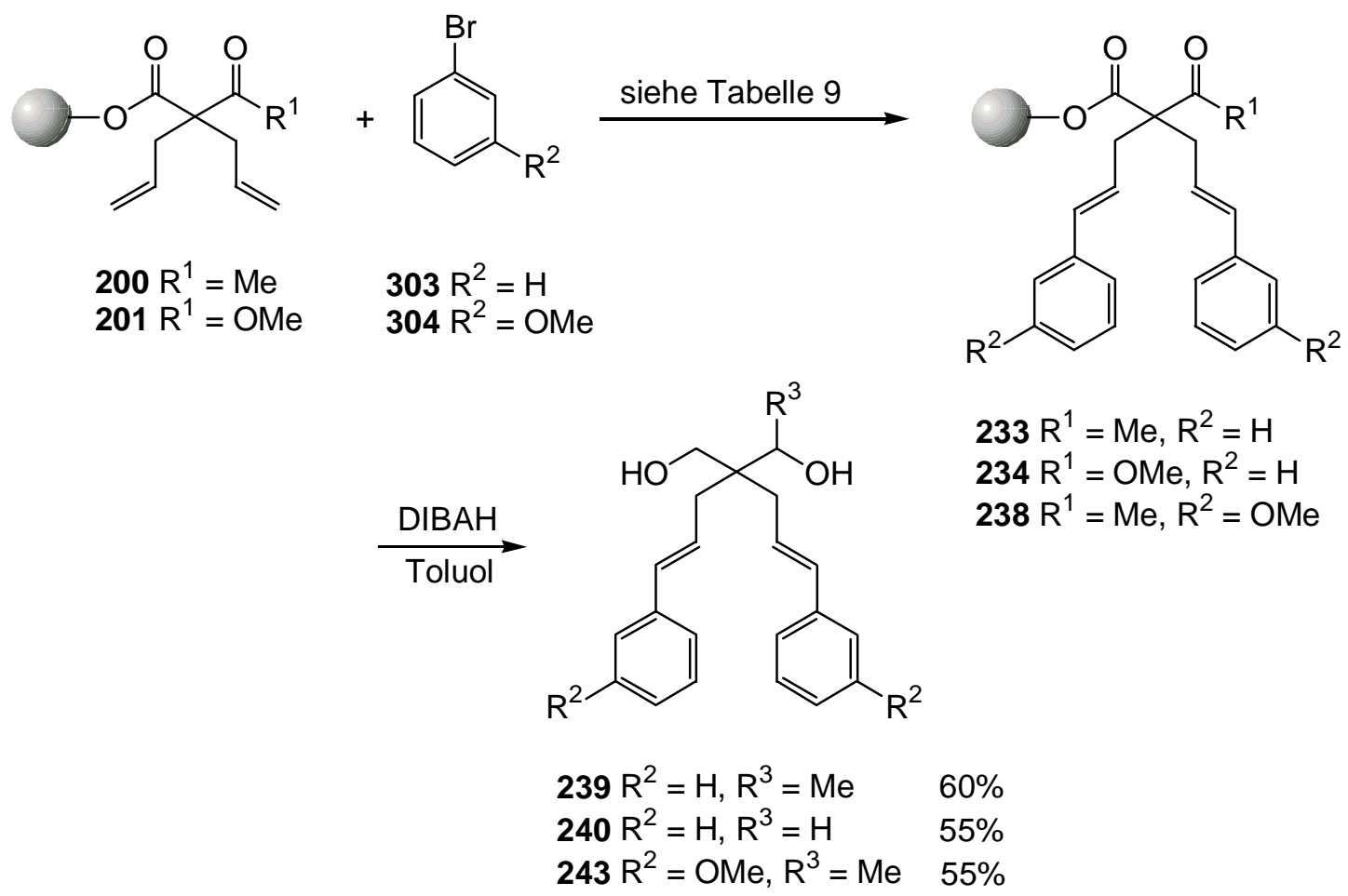

Auch mit den in Heck-Reaktionen weniger reaktiven Bromaromaten zeigt sich, daß trotz der Erhöhung der Anzahl der Synthesestufen die erhaltenen Gesamtausbeuten der Zielverbindungen vergleichbar oder sogar höher sind als bei den in Kapitel 6.3. beschriebenen allylischen Alkylierungen mit substituierten, aromatischen Allylacetaten. 


\begin{tabular}{|c|c|c|c|c|c|c|c|}
\hline Edukt & Bromid & Katalysator & Zusätze & LM & Bedingungen & Produkt & $\begin{array}{c}\text { Ausbeute } \\
(\%)\end{array}$ \\
\hline 200 & 303 & $\begin{array}{l}20 \mathrm{~mol} \% \\
\mathrm{Pd}(\mathrm{OAc})_{2}\end{array}$ & $\begin{array}{c}40 \mathrm{~mol}^{\%} \\
\mathrm{PPh}_{3} \\
3 \text { eq. } \mathrm{Net}_{3}\end{array}$ & $\mathrm{MeCN}$ & $80{ }^{\circ} \mathrm{C}, 5 \mathrm{~h}$ & 239 & 23 \\
\hline 200 & 303 & $10 \mathrm{~mol} \% 89$ & 3 eq. $\mathrm{NEt}_{3}$, & $\mathrm{DMF}$ & $110^{\circ} \mathrm{C}, 12 \mathrm{~h}$ & 239 & Spuren \\
\hline 200 & 303 & $10 \mathrm{~mol} \% 89$ & $\begin{array}{l}3 \text { eq. } \mathrm{KOAc}, \\
1 \text { eq. } \mathrm{Bu}_{4} \mathrm{NCl}\end{array}$ & DMF & $120^{\circ} \mathrm{C}, 12 \mathrm{~h}$ & 239 & 20 \\
\hline 200 & 303 & $\begin{array}{l}20 \mathrm{~mol} \% \\
\mathrm{Pd}(\mathrm{OAc})_{2}\end{array}$ & $\begin{array}{c}40 \mathrm{~mol}^{\%} \\
\mathrm{PPh}_{3}, \\
3 \text { eq. } \mathrm{KOAc}, \\
1 \text { eq. } \mathrm{Bu}_{4} \mathrm{NCl}\end{array}$ & DMF & $100^{\circ} \mathrm{C}, 22 \mathrm{~h}$ & 239 & 60 \\
\hline 201 & 303 & $\begin{array}{l}20 \mathrm{~mol} \% \\
\operatorname{Pd}(\mathrm{OAc})_{2}\end{array}$ & $\begin{array}{c}40 \mathrm{~mol}^{\%} \\
\mathrm{PPh}_{3}, \\
3 \text { eq. } \mathrm{KOAc}, \\
1 \text { eq. } \mathrm{Bu}_{4} \mathrm{NCl}\end{array}$ & $\mathrm{DMF}$ & $100^{\circ} \mathrm{C}, 22 \mathrm{~h}$ & 240 & 55 \\
\hline 200 & 304 & $\begin{array}{l}20 \mathrm{~mol} \% \\
\mathrm{Pd}(\mathrm{OAc})_{2}\end{array}$ & $\begin{array}{c}40 \mathrm{~mol}^{\%} \\
\mathrm{PPh}_{3} \\
3 \text { eq. } \mathrm{NBu}_{3}\end{array}$ & DMF & $100^{\circ} \mathrm{C}, 24 \mathrm{~h}$ & 243 & 55 \\
\hline
\end{tabular}

Tabelle 9: Heck-Reaktionen mit Arylbromiden.

Die Übertragung der Ergebnisse auf heteroaromatische Halogenide gelang nicht. Bei der Umsetzung von 2-Bromthiophen $\mathbf{3 0 5}$ mit dem polymergebundenen Diallylsystem 200 unter Palladiumacetat-Katalyse oder mit dem Palladacyclus 89 konnten nach der reduktiven Abspaltung von der festen Phase neben dem diallylierten 1,3-Diol 202 nur Spuren der Zielverbindung NMR-spektroskopisch nachgewiesen werden. Ähnliche Resultate wurden bei der Verwendung der entsprechenden Iodverbindung erhalten.<smiles>C=CCC(CC=C)(C(C)=O)C(=O)Oc1ccccc1</smiles>

200<smiles>Brc1cccs1</smiles>

1) siehe Tabelle 10

2) DIBAH, Toluol

305<smiles>CC(O)C(CO)(C/C=C/c1cccs1)C/C=C/c1cccs1</smiles> 


\begin{tabular}{|c|c|c|c|c|c|c|}
\hline Edukt & Bromid & Katalysator & Zusätze & LM & Bedingungen & $\begin{array}{c}\text { Ausbeute } \\
(\%)\end{array}$ \\
\hline 200 & 305 & $\begin{array}{l}20 \mathrm{~mol} \% \\
\mathrm{Pd}(\mathrm{OAc})_{2}\end{array}$ & $\begin{array}{c}40 \mathrm{Mol}_{\%} \mathrm{PPh}_{3}, \\
3 \text { eq. } \mathrm{NBu}_{3}\end{array}$ & $\mathrm{DMF}$ & $100{ }^{\circ} \mathrm{C}, 22 \mathrm{~h}$ & Spuren \\
\hline 200 & 305 & $\begin{array}{l}20 \mathrm{~mol} \% \\
\mathrm{Pd}(\mathrm{OAc})_{2}\end{array}$ & $\begin{array}{c}40 \mathrm{Mol}^{2} \mathrm{PPh}_{3}, \\
3 \text { eq. } \mathrm{KOAc}, \\
1 \text { eq. } \mathrm{Bu}_{4} \mathrm{NCl}\end{array}$ & DMF & $100{ }^{\circ} \mathrm{C}, 72 \mathrm{~h}$ & Spuren \\
\hline 200 & 305 & $\begin{array}{l}10 \text { mol\% } 89 \\
\text { (Beller-Kat.) }\end{array}$ & 3 eq. $\mathrm{NBu}_{3}$ & $\mathrm{DMF}$ & $120^{\circ} \mathrm{C}, 48 \mathrm{~h}$ & Spuren \\
\hline
\end{tabular}

Tabelle 10: Versuchte Heck-Reaktionen mit heteroaromatischen Arylhalogeniden.

\subsection{Diskussion ausgewählter spektroskopischer Daten}

Im ${ }^{1} \mathrm{H}-\mathrm{NMR}$-Spektrum der Verbindung 243 erkennt man bei $\delta=1.31$ das Dublett-Signal der 4- $\mathrm{H}_{3}$-Gruppe mit $J=6.2 \mathrm{~Hz}$. Die Resonanzen der diastereotopen 1'- $\mathrm{H}_{2}$-Protonen beobachtet man bei $\delta=2.09$ und $\delta=2.38$ jeweils als Duplett vom Duplett mit einer geminalen Kopplungskonstanten von $J=13.7 \mathrm{~Hz}$ und einer Kopplung zu 2'-H mit $J=7.6 \mathrm{~Hz}$, während die 1 ' $-\mathrm{H}_{2}$-Protonen als Dublett mit $J=7.6 \mathrm{~Hz}$ bei $\delta=2.46$ resonieren. Das Signal der 1-OHGruppe bei $\delta=2.68$ erscheint als Duplett mit einer Kopplung von $J=5.1 \mathrm{~Hz}$ zu der 1- $\mathrm{H}_{2}$-Gruppe, 3-OH hingegen resoniert als breites Singulett bei $\delta=2.77$. Die 1- $\mathrm{H}_{2}-$ Protonen sind wiederum diastereotop und zeigen eine Resonanz bei $\delta=3.65$ und $\delta=3.83$ als Dublett vom Duplett mit $J=11.3$ und $5.1 \mathrm{~Hz}$. Ein zentriertes Multiplett bei $\delta=3.97 \mathrm{kann}$ dem $3-\mathrm{H}$ zugeordnet werden. Die 2'-H- und 2''-H-Protonen erzeugen ein Signal bei $\delta=6.24$ und $\delta=6.28$ in Form eines Dubletts vom Triplett mit $J=15.5$ und 7.6 Hz, während die 3'-H- und 3"'-H-Protonen bei $\delta=6.44$ und $\delta=6.45$ als Dubletts mit einer trans-Kopplung zu 2'/2"'-H mit $J=15.5 \mathrm{~Hz}$ resonieren. Die aromatischen 7'-H und 7' -H erkennt man als Dublett mit $J=8.2 \mathrm{~Hz}$ bei $\delta=6.77$, die 5 '-H und 5 ''-H bei $\delta=6.88$ als Singulett. Die zur MethoxyGruppe para-ständigen Protonen resonieren bei $\delta=6.95$ als Dublett mit $J=8.2 \mathrm{~Hz}$ sowie bei $\delta=7.22$ die $m e t a$-ständigen Protonen als Triplett mit $J=8.2 \mathrm{~Hz}$.

Das ${ }^{13}$ C-NMR-Spektrum zeigt im Hochfeld bei $\delta=18.01$ das Signal von C-4 und bei $\delta=34.51$ und 36.15 die Resonanzen von C-1' und C-1',. Die Signale von C-2, C-1 und C-3 beobachtet man bei $\delta=44.88,55.16$ und 73.57. Die aromatischen C-5' und C-5' resonieren bei $\delta=111.5$, C-7' und C-7' ' bei $\delta=112.6$, während die Signale von C-9' und C-9', bei $\delta=118.7$ zu sehen sind. Die olefinischen Kohlenstoffatome zeigen eine Resonanz bei $\delta=126.3$ für C-3' und C-3' ' sowie bei $\delta=129.5$ für C-2' und C-2'". Die quartären C-4'/C-4', bzw. C-6'/C-6', geben ein Signal bei $\delta=138.8$ bzw. 159.7 . 
Das IR-Spektrum zeigt bei $3356 \mathrm{~m}^{-1}$ die Bande der OH-Gruppen und bei 3026 und $2937 \mathrm{~cm}^{-1}$ die C-H-Streckschwingungen. Bei 1599 und $1579 \mathrm{~cm}^{-1}$ erkennt man die Banden der olefinischen Bindungen und bei 1454 und $1377 \mathrm{~cm}^{-1}$ die der $\mathrm{CH}_{3}$-Deformationsschwingung.

Das DCI-Massenspektrum zeigt die Massenpeaks $M+18$ und $2 \mathrm{M}+18$ bei m/z = 400 und 782 . 


\section{Festphasensynthese von Pyrazolonen}

Pyrazolone und ihre Derivate stellen eine wichtige Substanzklasse von Heterocyclen dar, die dank ihrer vielfältigen biologischen Aktivität reges Interesse der synthetischen Chemiker ${ }^{[65]}$ hervorgerufen und Verwendung in einer ganzen Reihe von Medikamenten gefunden haben, z. B. als Analgetika, Antiphlogistika, Antirheumatika und Antipyretika. ${ }^{[31]}$

L. F. Tietze und A. Steinmetz ${ }^{[35]}$ erarbeiteten eine flexible Synthese von Pyrazolonen an der festen Phase ausgehend von polymergebundenem Acetoacetat 138. Nach selektiven Alkylierungen in $\alpha$ - und $\gamma$-Position wurden mit Phenylhydrazin 308 die entsprechenden Hydrazone 309 aufgebaut, die durch cyclisierende Abspaltung die gewünschten Pyrazolone 310 in Ausbeuten von 40 - 95\% lieferten. Die hohe Reinheit der Produkte von > 90\% erklärt sich aus der Tatsache, daß nur für die Cyclisierung geeignete Strukturen vom polymeren Träger abgespalten werden können, Verunreinigungen jedoch verbleiben an der festen Phase.<smiles>CC(=O)CC(=O)Oc1ccccc1</smiles>

138

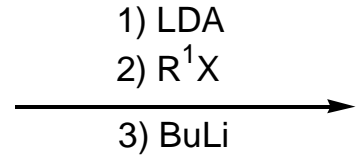

4) $R^{2} X$

5) $\mathrm{H}^{+}$<smiles>[R]C([R])C(=O)CC(=O)Oc1ccccc1</smiles>

306<smiles>[R7]C([R7])C(=N)C([R7])C([R7])C(=O)Oc1ccccc1</smiles>

307

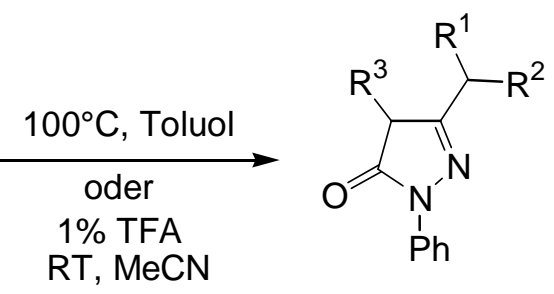

310

Bei den durchgeführten Synthesen wurde stets Phenylhydrazin 308 eingesetzt. Nach der Modifikation der übrigen Positionen des Pyrazolon-Ringes soll nun die Hydrazin-Komponente variiert werden, um eine maximale Diversität der synthetisierten Heterocyclen zu gewährleisten. Hierzu wurden eine Reihe unterschiedlich substituierter Phenylhydrazine, heteroaromatische sowie aliphatische Hydrazine eingesetzt. ${ }^{[66]}$ Die Hydrazon-Bildung erfolgte ausgehend von polymergebundenem Acetoacetat 138 durch Umsetzung mit 10 Äquivalenten des entsprechen- 
den Hydrazins in Tetrahydrofuran für 5 Stunden bei Raumtemperatur. Die cyclisierende Abspaltung gelang in einer 1\%igen Lösung von Trifluoressigsäure in Acetonitril innerhalb von zwei Stunden bei Raumtemperatur.

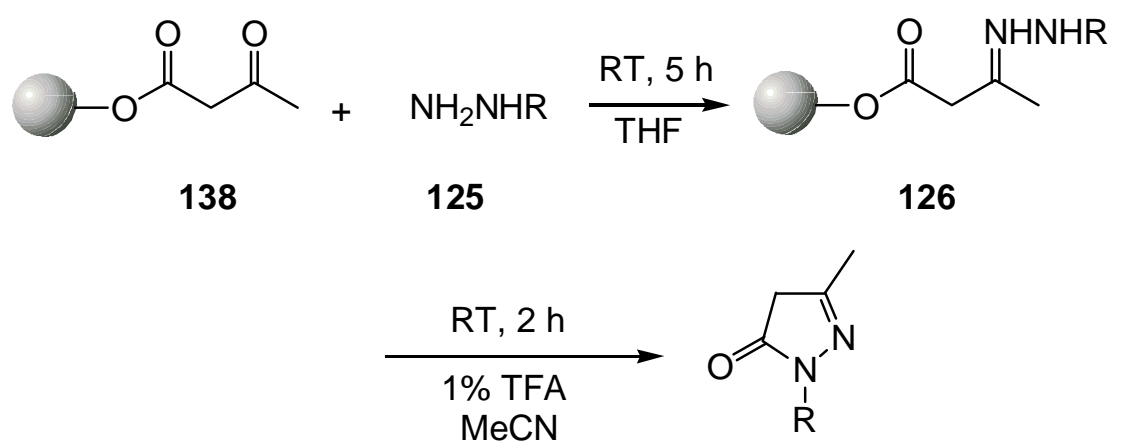

127

Wie aus Tabelle 11 ersichtlich wurden die Pyrazolone 316-320 in guten bis sehr guten Gesamtausbeuten von 49 - 96\% über drei Stufen erhalten. Neben den para-substituierten Phenylhydrazinen 311-313 reagierten auch das heteroaromatische Hydrazin 314 sowie das Trifluorethylhydrazin 315 glatt zu den gewünschten Produkten, die Reinheiten von > 90\% aufwiesen. 


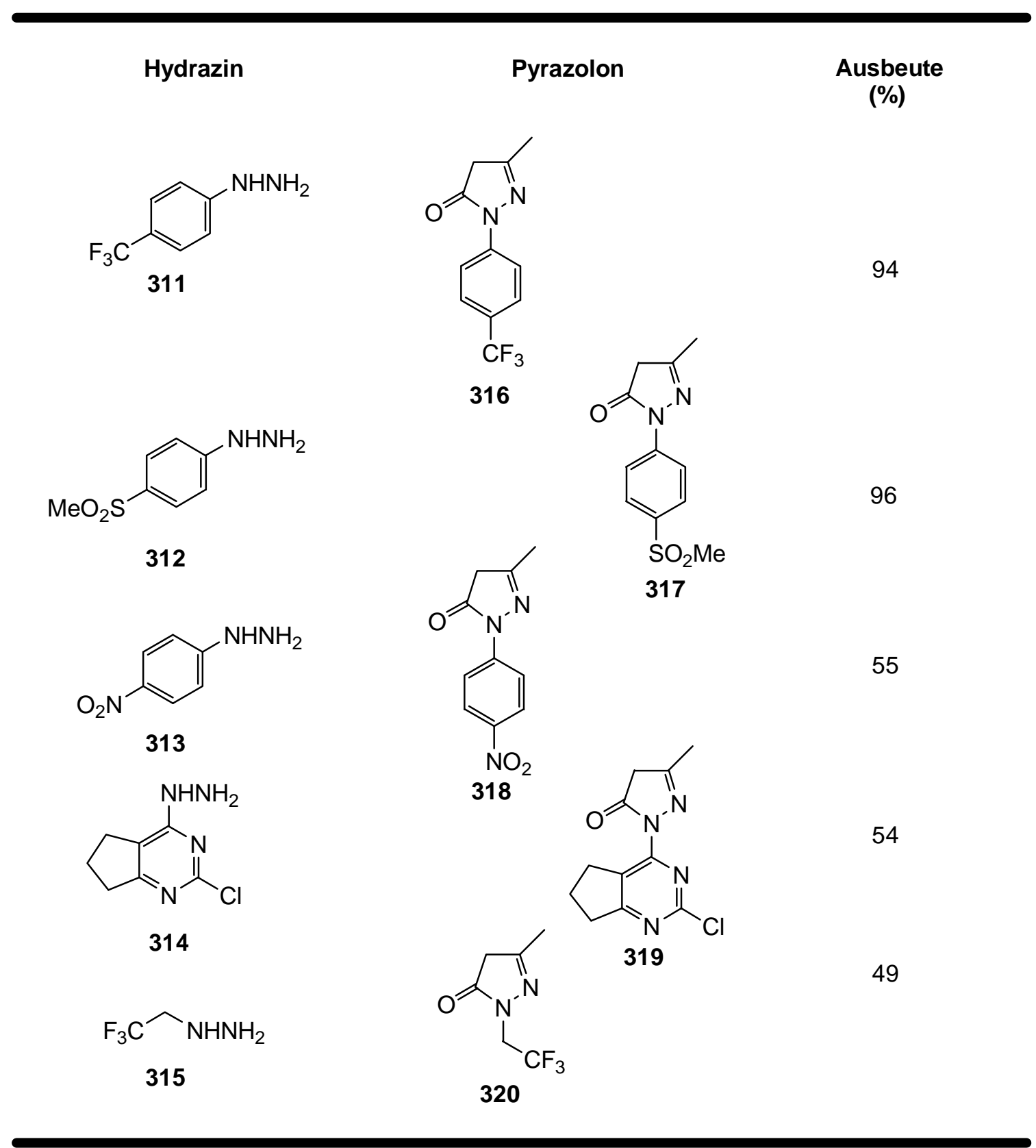

Tabelle 11: Synthetisierte Pyrazolone 316-320.

Das ortho-nitrophenyl-substituierte Hydrazon 322 konnte weder Trifluoressigsäure-katalysiert bei Raumtemperatur noch thermisch bei $100{ }^{\circ} \mathrm{C}$ cyclisiert werden. Dies gelang jedoch durch Erwärmen von 322 in einer 1\%igen Lösung von Trifluoressigsäure in Toluol auf $80^{\circ} \mathrm{C}$ für 5 Stunden und man erhielt $\mathbf{3 2 3}$ in 57\%iger Ausbeute. Trotz der verschärften Reaktionsbedingungen lieferte die Abspaltung von der festen Phase das Produkt in der erwarteten Reinheit. 


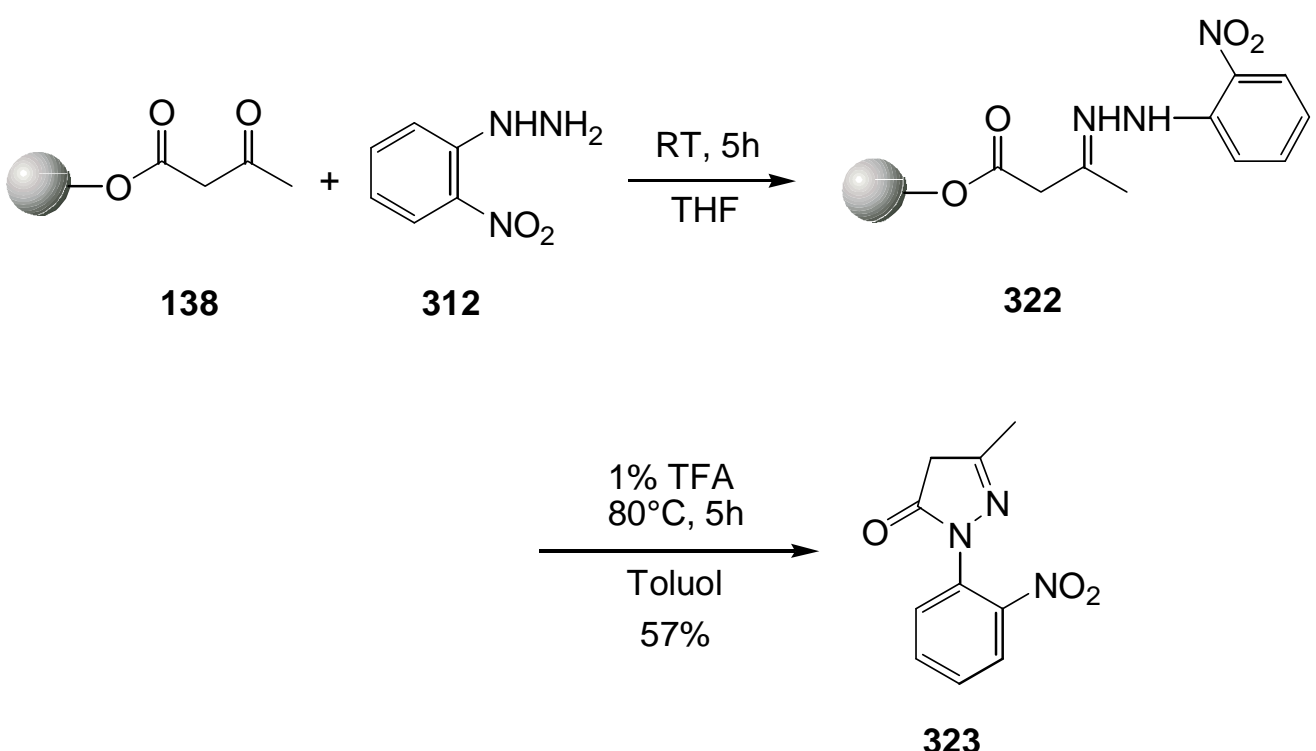

Neben den Hydrazinen 311-315, 321 wurden auch die Hydrazin-Hydrochloride 324-326 für die Heterocyclen-Synthese am polymeren Träger eingesetzt. Es zeigte sich, daß die Hydrazine vor der Reaktion mit dem polymergebundenen Acetoacetat 138 aus den entsprechenden Salzen freigesetzt werden müssen. Dies geschah durch Waschen der Hydrochloride mit gesättigter Natriumhydrogencarbonat-Lösung und anschließender Extraktion mit Essigester. Die organische Phase wurde getrocknet und nach dem Einengen im Vakuum setzte man die Hydrazine direkt mit polymergebundenem Acetoacetat 138 um. Nach Abspaltung von der festen Phase erhielt man die Pyrazolone 327-329 in Gesamtausbeuten von 45 - 63\% (Tabelle 12). In diesen Fällen bereitete eine ortho-Substitution des Phenylringes oder eine sekundäres Zentrum in 1'Position keine Probleme bei der Cyclisierung zum Pyrazolon. 
Hydrazin<smiles>NNc1ccc(F)cc1F</smiles>

324<smiles>CC(NN)c1ccccc1</smiles>

325<smiles>CC1CCCCC1NN</smiles>

326
Pyrazolon<smiles>CC1=NN(c2ccc(F)cc2F)C(=O)C1</smiles>

327<smiles>CC1=NN(C(C)c2ccccc2)C(=O)C1</smiles>

328

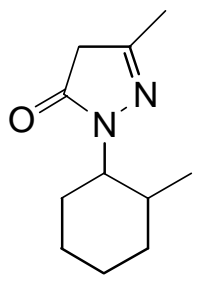

329
Ausbeute

(\%)

50

63

45

\section{Tabelle 12: Synthetisierte Pyrazolone 327-329.}

Die Pyrazolone 328 und 329 wurden als Gemische aus OH- und Keto-Form ${ }^{[67]}$ erhalten. Nach ${ }^{1}$ H-NMR-spektroskopischer Untersuchung liegt das Verhältnis im Falle von 328 bei 2.5 : 1, im Falle von 329 bei $2: 1$.

Einige der verwendeten Hydrazine ließen sich nicht für die Festphasensynthese von Pyrazolonen einsetzen. Während das Benzolthiazol 333 kein polymergebundenes Hydrazon bildete, gelang die Cyclisierung der aus den stark elektronenarmen Acrylsäureester- und Thiosemicarbazid-Derivate 330 bzw. 331 sowie dem Benzolsulfonsäurehydrazid 332 gebildeten Hydrazone nicht, weder bei Raumtemperatur noch bei $80{ }^{\circ} \mathrm{C}$. Vermutlich ist die Nucleophile des Stickstoffs durch die elektronenziehenden Substituenten zu stark abgesenkt. 
<smiles>COC(=O)/C(C#N)=C\N</smiles>

330<smiles>CCNC(=S)NN</smiles>

331<smiles>NNS(=O)(=O)c1ccccc1</smiles>

332<smiles>Cc1cccc2sc(NN)nc12</smiles>

333

\subsection{Diskussion ausgewählter spektroskopischer Daten}

Im ${ }^{1} \mathrm{H}-\mathrm{NMR}-$ Spektrum der Verbindung 317 erkennt man bei $\delta=2.23$ das Signal der MethylGruppe an C-3 als Singulett, während die Schwefel-gebundene Methyl-Gruppe bei $\delta=3.06$ ebenfalls als Singulett resoniert. Die Resonanz der Methylen-Gruppe des Heterocyclus erscheint bei $\delta=3.50$ als Singulett. Im Tieffeld beobachtet man zwei Dublett-Signale mit jeweils $J=9.4 \mathrm{~Hz}$ der aromatischen 3'-H und 5'-H bei $\delta=7.93$ bzw. von 2'-H und 6'-H bei $\delta=8.15$. Das ${ }^{13}$ C-NMR-Spektrum zeigt bei $\delta=17.02$ das Signal der Methyl-Gruppe an C-3 und bei $\delta=43.08$ das Signal von C-4. Die Sulfonylmethyl-Gruppe resoniert bei $\delta=44.59$. Die Resonanzen der aromatischen C-2' und C-6' bzw. C-3' und C-5' beobachtet man bei $\delta=118.1$ bzw. $\delta=128.3$, während die quartären Kohlenstoffatome C-4' und C-1' bei $\delta=135.4$ und $\delta=142.2$ resonieren. Die Signale von C-3 und C-5 erscheinen bei $\delta=157.5$ und $\delta=170.9$.

Im IR-Spektrum zeigen sich $\mathrm{CH}$-Streckschwingungen bei $2920 \mathrm{~cm}^{-1}, \mathrm{CH}_{3}$-Deformationsschwingungen werden bei 1451 und $1360 \mathrm{~cm}^{-1}$ beobachtet. Besonders charakteristisch sind die Banden bei 1716 und $1598 \mathrm{~cm}^{-1}$, die der Carbonyl-Gruppe bzw. der olefinischen Bindungen zugeordnet werden können. Des weiteren kann das Signal bei $820 \mathrm{~cm}^{-1}$ als $\mathrm{CH}$-Deformationsschwingung eines 1,4-disubstituierten Benzolringes interpretiert werden.

Im EI-Massenspektrum erkennt man neben dem Molpeak bei $\mathrm{m} / \mathrm{z}=252$, der gleichzeitig den Basispeak darstellt. Molekülfragmente bei $\mathrm{m} / \mathrm{z}=237$ und $\mathrm{m} / \mathrm{z}=173$ entsprechend der $\mathrm{Ab}$ spaltung eine Methyl-Gruppe bzw. der Sulfonyl-Gruppe. Schließlich kann das Fragment bei $\mathrm{m} / \mathrm{z}=155$ durch den Verlust der Methylsulfonylphenyl-Einheit erklärt werden. 


\section{Zusammenfassung}

Die Festphasensynthese verknüpft mit kombinatorischen Ansätzen ist ein wichtiges Instrument zur Entwicklung neuer pharmakologischer Wirkstoffe. Durch die Verwendung polymergebundener Verbindungen gelang die Automatisierung vieler Reaktionschritte, wodurch sich die Anzahl der Syntheseprodukte pro Zeiteinheit stark steigern ließ. Die erhaltenen Substanzbibliotheken lassen sich mit Hilfe moderner Screening-Verfahren sehr effizient und mit hohem Durchsatz auf ihre biologische Aktivität hin untersuchen. Die direkte Übertragung von Synthesemethode, die in Lösung entwickelt wurden, an die feste Phase ist nur in Ausnahmefällen möglich. Es besteht daher ein großer Bedarf an der Entwicklung neuer Transformationen am polymeren Träger.

In der vorliegenden Arbeit konnte die Palladium-katalysierte allylische Alkylierung an der festen Phase etabliert werden. Durch den einfachen Zugang zu einer Fülle von AllylKomponenten und der hohen Toleranz gegenüber einer Vielzahl funktioneller Gruppen ist diese Reaktion von großem Interesse für die Kombinatorische Chemie.

Die Synthese der polymergebundenen 1,3-Dicarbonylverbindungen 138 und 143 gelang durch Umsetzung des spacer-modifizierten Merrifield-Harzes 6 mit Acetessigsäure-t-butylester 135 bzw. Malonsäuremonomethylesterchlorid 142.

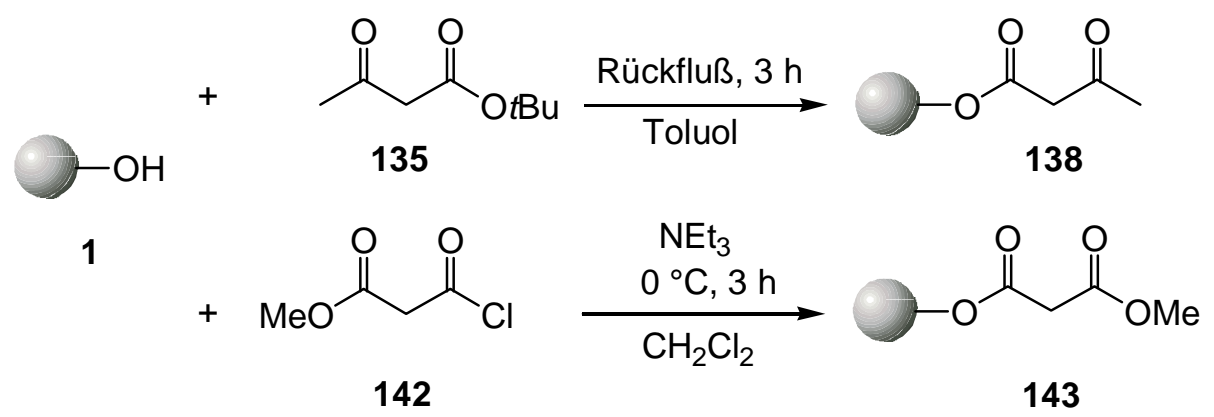

Die verwendeten Allylacetate konnten durch Acetylierung von Allylalkoholen synthetisiert werden, welche durch Reduktion von $\alpha, \beta$-ungesättigten Carbonylverbindungen sowie durch Grignard-Reaktion von Allylmagnesiumbromid mit Aldehyden erhalten wurden. Es ließen sich auf diese Weise unterschiedlich substituierte aliphatische, cyclische sowie aromatisch- und heteroaromatisch-substituierte Allylacetate in Ausbeuten von 21 - 99\% herstellen. 


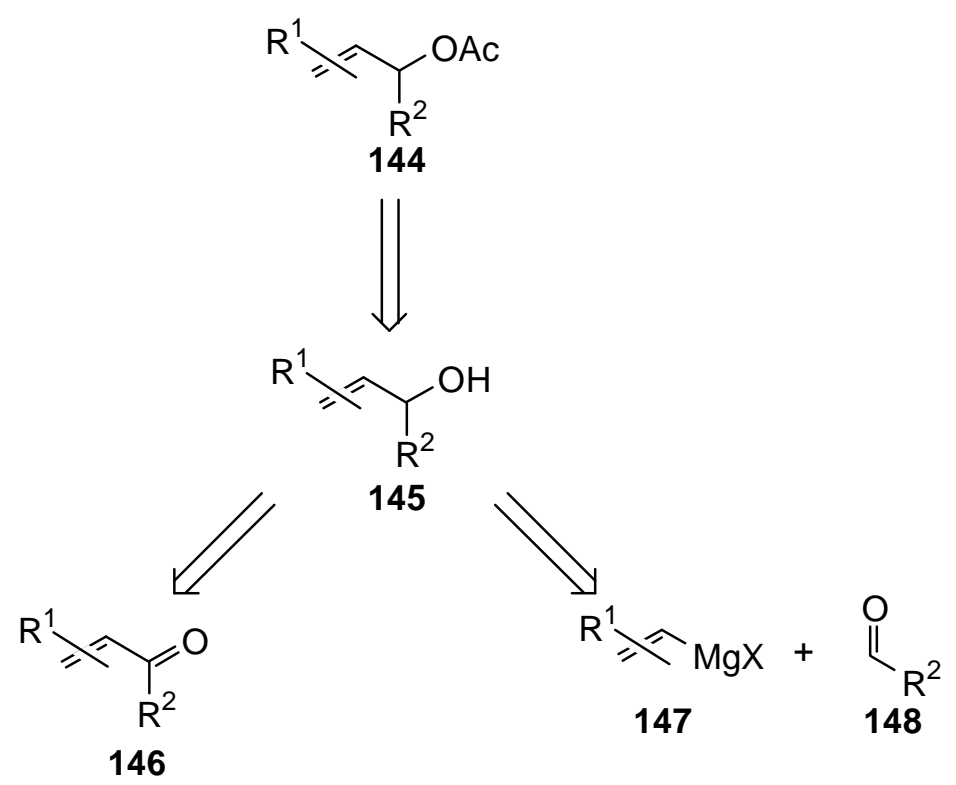

Die aliphatischen Allylacetate 156-158,199 wurden mit polymergebundenem Acetoacetat 138 und Methylmalonat 143 unter Zusatz von 20 mol\% Tetrakis(triphenylphosphan)palladium und dem Basensystem N,O-Bis(trimethylsilyl)acetamid (BSA)/Kaliumacetat unter Rückflußbedingungen oder bei Raumtemperatur in THF umgesetzt. Nach reduktiver Abspaltung von der festen Phase mit Diisobutylaluminiumhydrid (DIBAH) erhielt man die dialkylierten 1,3-Diole 202,203,210-212 in guten bis sehr guten Gesamtausbeuten von 45 - 88\% über drei Stufen ausgehend von der Konzentration freier Hydroxy-Gruppen in spacermodifizierten Merrifield-Harz 6. Im Falle des hochsubstituierten Allylacetats 159 ließ sich lediglich das monoalkylierte Produkt 213 in 8\%iger Ausbeute isolieren.
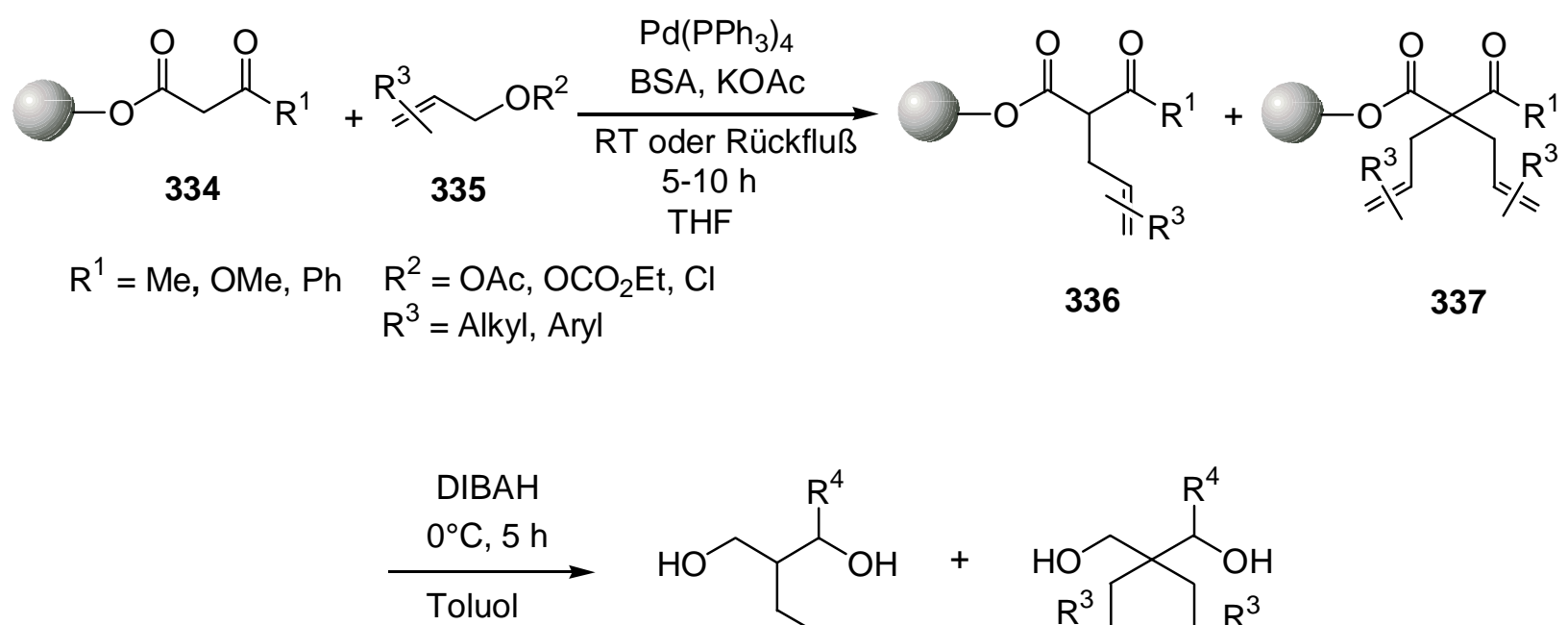<smiles>[R]C(O)C(CO)CC=C</smiles>

338

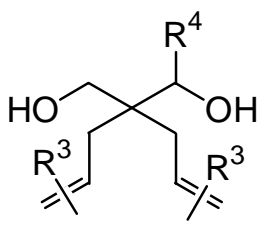

$\mathrm{R}^{4}=\mathrm{Me}, \mathrm{H}, \mathrm{Ph}$ 


\begin{tabular}{|c|c|c|c|c|}
\hline Allylacetat & $\mathrm{R}^{3}$ & Produkt & $\mathrm{R}^{4}$ & Ausbeute (\%) \\
\hline 199 & $\mathrm{H}$ & 202 & $\mathrm{Me}$ & $88^{\mathrm{a}}$ \\
\hline 199 & $\mathrm{H}$ & 203 & $\mathrm{H}$ & $68^{\mathrm{a}}$ \\
\hline 156 & 3-Me & 210 & $\mathrm{Me}$ & $39^{\mathrm{a}}$ \\
\hline 157 & 1-Me & 210 & $\mathrm{Me}$ & $39^{\mathrm{a}}$ \\
\hline 158 & 2-Me & 211 & $\mathrm{Me}$ & $60^{\mathrm{a}}$ \\
\hline 158 & 2-Me & 212 & $\mathrm{H}$ & $45^{\mathrm{a}}$ \\
\hline 159 & 3-Me, 3-R & 213 & $\mathrm{Me}$ & $8^{\mathrm{b}}$ \\
\hline 199 & $\mathrm{H}$ & 219 & $\mathrm{Ph}$ & $73^{\mathrm{a}}$ \\
\hline 175 & cycl-Hexenyl & 227 & $\mathrm{Me}$ & $59^{\mathrm{b}}$ \\
\hline 175 & cycl-Hexenyl & 228 & $\mathrm{H}$ & $40^{\mathrm{b}}$ \\
\hline 176 & cycl-Pentenyl & 229 & $\mathrm{Me}$ & $49^{b}$ \\
\hline 176 & cycl-Pentenyl & 230 & $\mathrm{H}$ & $53^{\mathrm{b}}$ \\
\hline 177 & $\begin{array}{c}\text { 2-Me- } \\
\text { cycl-Pentenyl }\end{array}$ & 231 & $\mathrm{Me}$ & $19^{\mathrm{b}}$ \\
\hline 178 & $\begin{array}{c}\text { 3-Me- } \\
\text { cycl-Pentenyl }\end{array}$ & 232 & $\mathrm{Me}$ & $28^{\mathrm{b}}$ \\
\hline 178 & $\begin{array}{c}\text { 3-Me- } \\
\text { cycl-Pentenyl }\end{array}$ & 233 & $\mathrm{H}$ & $14^{\mathrm{b}}$ \\
\hline
\end{tabular}

\footnotetext{
${ }^{\mathrm{a}}$ Dialkyliert.

${ }^{\mathrm{b}}$ Monoalkyliert.
}

Tabelle 13: Synthetisierte Diole ausgehend von aliphatischen und cyclischen Allylacetaten.

Die Umsetzungen mit den cyclische Allylacetaten 175-178 lieferten auch bei Verwendung hoher Überschüsse und längerer Reaktionszeiten ausschließlich die monoalkylierten 1,3-Diole 227-233 in Ausbeuten von 14 - 59\%.

Die aromatisch- bzw heteroaromatisch-substituierten Allylacetate 130, 179, 180, 190, 191 lieferten die dialkylkierten 1,3-Diole 239-243, 251-254 in Gesamtausbeuten von 14 - 57\%, bei Verwendung des 1,3-disubstituierten Acetats 161 wurde nur das monoalkylierte Diol 246 erhalten. 


\begin{tabular}{|c|c|c|c|c|}
\hline Allylacetat & $\mathrm{R}^{3}$ & Produkt & $\mathrm{R}^{4}$ & Ausbeute (\%) \\
\hline $\mathbf{1 6 0}$ & $3-\mathrm{Ph}$ & $\mathbf{2 3 9}$ & $\mathrm{Me}$ & $52^{\mathrm{a}}$ \\
\hline $\mathbf{1 6 0}$ & $3-\mathrm{Ph}$ & $\mathbf{2 4 0}$ & $\mathrm{H}$ & $57^{\mathrm{a}}$ \\
\hline $\mathbf{1 6 1}$ & 1-Et-3-Ph & $\mathbf{2 4 6}$ & $\mathrm{Me}$ & $27^{\mathrm{b}}$ \\
\hline $\mathbf{1 7 9}$ & 3-(4-NMe $)-\mathrm{Ph}$ & $\mathbf{2 4 1}$ & $\mathrm{Me}$ & $34^{\mathrm{a}}$ \\
\hline $\mathbf{1 7 9}$ & 3-(4-NMe 2$)-\mathrm{Ph}$ & $\mathbf{2 4 2}$ & $\mathrm{H}$ & $18^{\mathrm{a}}$ \\
\hline $\mathbf{1 8 0}$ & 3-(3-OMe)-Ph & $\mathbf{2 4 3}$ & $\mathrm{Me}$ & $23^{\mathrm{a}}$ \\
\hline $\mathbf{1 8 0}$ & 3-(3-OMe)-Ph & $\mathbf{2 4 4}$ & $\mathrm{H}$ & $24^{\mathrm{b}}$ \\
\hline $\mathbf{1 9 0}$ & 1-(3-Furyl) & $\mathbf{2 5 1}$ & $\mathrm{Me}$ & $23^{\mathrm{a}}$ \\
\hline $\mathbf{1 9 0}$ & 1-(3-Furyl) & $\mathbf{2 5 2}$ & $\mathrm{H}$ & $20^{\mathrm{a}}$ \\
\hline $\mathbf{1 9 1}$ & 1-(2-Thiophenyl) & $\mathbf{2 5 3}$ & $\mathrm{Me}$ & $20^{\mathrm{a}}$ \\
\hline $\mathbf{1 9 1}$ & 1-(2-Thiophenyl) & $\mathbf{2 5 4}$ & $\mathrm{H}$ & $14^{\mathrm{a}}$ \\
\hline
\end{tabular}

${ }^{\mathrm{a}}$ Dialkyliert.

${ }^{\mathrm{b}}$ Monoalkyliert.

Tabelle 14: Synthetisierte 1,3-Diole ausgehend von aromatischen Allylacetaten.

Neben Allylacetaten konnten auch Allylcarbonate und -chloride für allylische Alkylierungen an der festen Phase eingesetzt werden. Die Carbonate 196 und 198 wurden ohne den Zusatz einer Base mit polymergebundenem Acetoacetat 138 bei Raumtemperatur umgesetzt, und man erhielt die dialkylierten Produkte in guten Ausbeuten von 68 bzw. 75\%. Die Verwendung von $n$ Buthyllithium zur Deprotonierung des festphasengebundenen Nucleophils und anschließende Alkylierung mit dem Allylchlorid 255 ergab ein Gemisch aus mono- und dialkyliertem 1,3-Diol.<smiles>CC(=O)CC(=O)Oc1ccccc1</smiles>

138<smiles>[CH2+]C(=O)CC(=O)Oc1ccccc1</smiles>
138<smiles>C=CCOC(=O)OCC</smiles>

196<smiles>CCOC(=O)OC/C=C/c1ccccc1</smiles>

1) $\mathrm{Pd}\left(\mathrm{PPh}_{3}\right)_{4}$ RT, $16 \mathrm{~h}$, THF

2) DIBAH, Toluol $68 \%$<smiles>C=CCC(CO)(CC=C)C(C)O</smiles>
202
1) $\mathrm{Pd}\left(\mathrm{PPh}_{3}\right)_{4}$, RT, $16 \mathrm{~h}$, THF
2) DIBAH, Toluol $75 \%$ 
<smiles>CCC(C)=C[C+]S(=O)(=O)c1ccccc1</smiles>

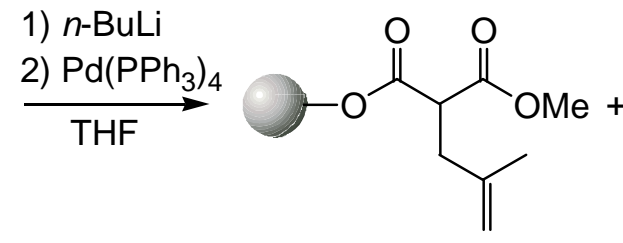

256<smiles>C=C(C)CC(CC(=C)C)(C(=O)OC)C(=O)Oc1ccccc1</smiles>

208<smiles>C=C(C)CC(CO)CO</smiles>

257<smiles>C=C(C)CC(CO)(CO)CC(=C)C</smiles>

212

Die Abspaltung der polymergebundenen Reaktionsprodukte von der festen Phase erfolgte meist durch Reduktion des Esterfunktionalität zum Alkohol. Als weitere Abspaltungsmethoden konnten auch die basische Umesterung und die Verseifung des festphasengebundenen Esters etabliert werden, so daß ebenfalls Ester und Carbonsäuren als Endprodukte zugänglich sind. Damit läßt sich die Diversität der Produkte im Abspaltungsschritt, der oft durch den verwendeten Linker vorgegeben ist, stark erhöhen.<smiles>CCCCC(=O)O</smiles>
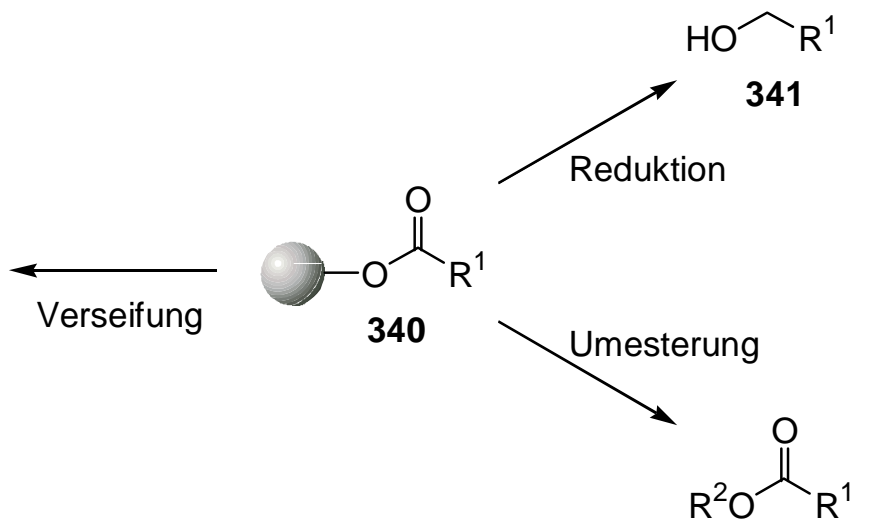

342

Neben den einfachen Allylcarbonaten ließen sich auch funktionalisierte Allylcarbonate vom Typ 275 einsetzten, die die Bildung von $\pi$-Allyl-Palladium-Komplexen an der festen Phase erlauben. Nachfolgende Umsetzung mit Nucleophilen ergab nach basischer Abspaltung vom polymeren Träger die gewünschten Verbindungen in Ausbeuten von 40 - 50\%. Die Synthese gelang sowohl im Zuge eines Zwei-Stufen-Prozesses unter Verwendung des Monocarbonats 275 mit anschließender Acetylierung des polymergebundenen Allylalkohols 273 als auch in einer einstufigen Reaktionsführung mit dem Dicarbonat 276. Besonders letztere Methode eignet sich zur schnellen und effizienten C-C-Verknüpfung an der festen Phase. 

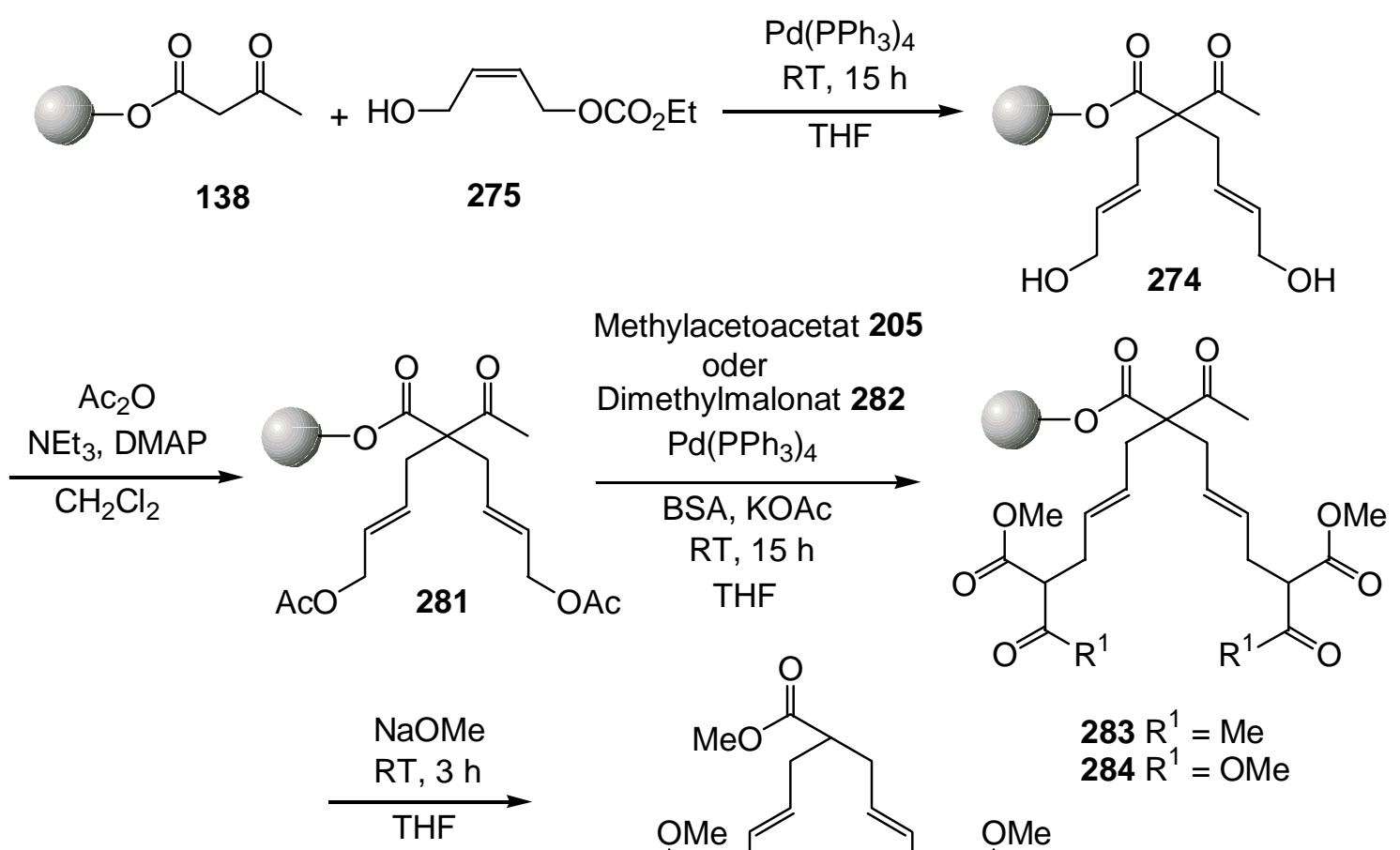<smiles>[R]C(=O)C(C/C=C/CC(C/C=C/CC(C(=O)OC)C(=O)OC)C(=O)OC)C(=O)OC</smiles>

Neben dem spacermodifizierten Merrifield-Harz 6 wurde auch das Wang-Harz 3 in den entwickelten Synthese-Sequenzen eingesetzt. Hierbei erfolgte die Abspaltung mit Trifluoressigsäure, die bei den verwendeten Acetoacetaten $\beta$-Ketosäuren lieferte, welche zu den entsprechenden Ketonen decarboxylierten. Es handelt sich hierbei um ein einfaches Beispiel eines „traceless“ Linkers. Durch Allylierung und nachfolgende Abspaltung vom polymeren Träger konnten die Ketone 290, 291 und 295 in Ausbeuten von 60 - 72\% erhalten werden. 
<smiles>[CH2+]C(=O)CC(=O)Oc1ccccc1</smiles>

289<smiles>C=CCOC(C)=O</smiles>

199

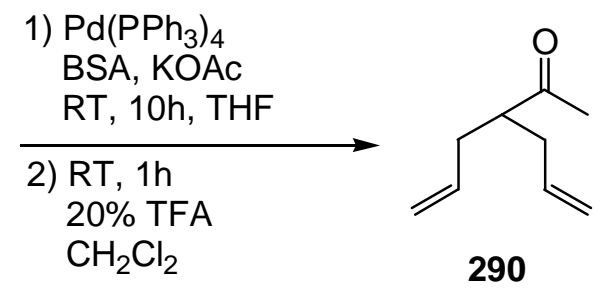

$72 \%$<smiles>CCOC(=O)OC/C=C/c1ccccc1</smiles>

1) $\mathrm{Pd}\left(\mathrm{PPh}_{3}\right)_{4}$ RT, 5h, THF

2) $\mathrm{RT}, 1 \mathrm{~h}$
$20 \% \mathrm{TFA}$
$\mathrm{CH}_{2} \mathrm{Cl}_{2}$

$60 \%$<smiles>CC(=O)C(C/C=C/c1ccccc1)C/C=C/c1ccccc1</smiles>

291<smiles>CC(=O)CC(=O)Oc1ccccc1</smiles>

289

1) $275, \mathrm{Pd}\left(\mathrm{PPh}_{3}\right)_{4}$ 2) $\mathrm{Ac}_{2} \mathrm{O}$

3) 205, $\mathrm{Pd}\left(\mathrm{PPh}_{3}\right)_{4}$

4) TFA<smiles>COC(=O)C(C/C=C/CC(C/C=C/CC(C(C)=O)C(C)=O)C(C)=O)C(C)=O</smiles>

295

$66 \%$

Die durch allylische Alkylierung erhaltenen, polymergebundenen Olefine konnten in einer Heck-Reaktion weiter umgesetzt werden. Sowohl Aryl-Iodide als auch Aryl-Bromide konnten eingesetzt werden, und man erhielt die entsprechenden 1,3-Diole in guten bis sehr guten Gesamtausbeute von 43 - 64\%. Die Ausbeuten dieser vierstufigen Sequenz lagen höher als bei der Umsetzung mit aromatisch-substituierten Allyacetaten in einer Drei-Stufen-Synthese.

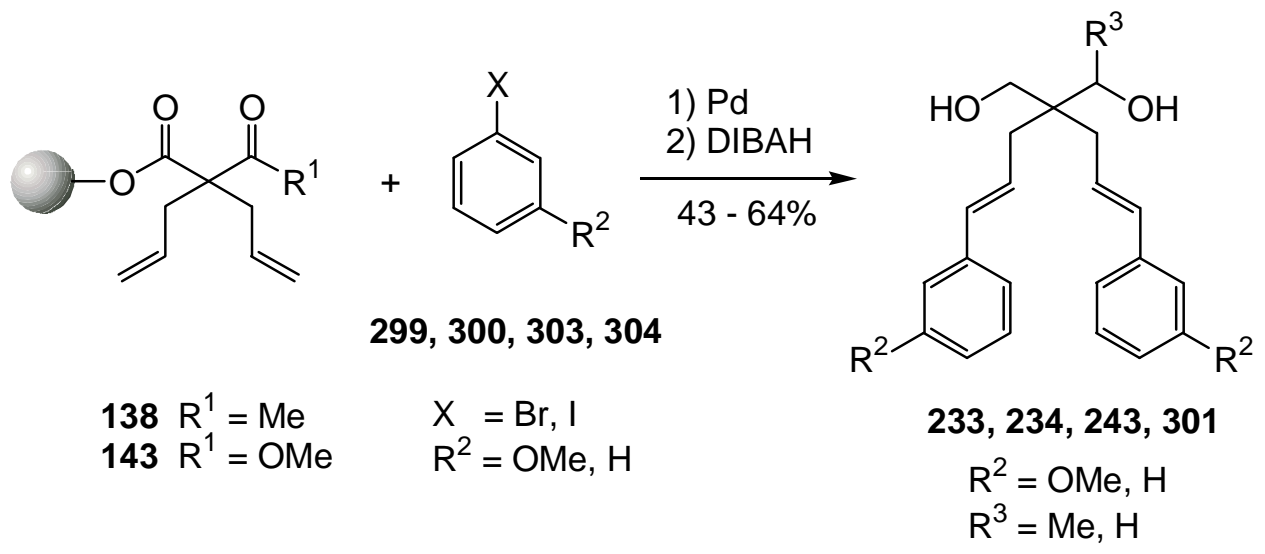




\begin{tabular}{|c|c|c|c|c|c|}
\hline Arylhalogenid & $\mathrm{X}$ & $\mathrm{R}^{2}$ & Produkt & $\mathrm{R}^{3}$ & Ausbeute (\%) \\
\hline $\mathbf{2 9 9}$ & $\mathrm{I}$ & $\mathrm{H}$ & $\mathbf{2 3 9}$ & $\mathrm{Me}$ & 64 \\
\hline $\mathbf{3 0 0}$ & $\mathrm{I}$ & $\mathrm{OMe}$ & $\mathbf{2 4 3}$ & $\mathrm{Me}$ & 53 \\
\hline $\mathbf{3 0 0}$ & $\mathrm{I}$ & $\mathrm{OMe}$ & $\mathbf{3 0 2}$ & $\mathrm{H}$ & 43 \\
\hline $\mathbf{3 0 3}$ & $\mathrm{Br}$ & $\mathrm{H}$ & $\mathbf{2 3 9}$ & $\mathrm{Me}$ & 60 \\
\hline $\mathbf{3 0 3}$ & $\mathrm{Br}$ & $\mathrm{H}$ & $\mathbf{2 4 0}$ & $\mathrm{H}$ & 55 \\
\hline $\mathbf{3 0 4}$ & $\mathrm{Br}$ & $\mathrm{OMe}$ & $\mathbf{2 4 3}$ & $\mathrm{Me}$ & 55 \\
\hline
\end{tabular}

Tabelle 15: Synthetisierte Diole

In einem weiteren Teil der vorliegenden Arbeit wurde die Festphasensynthese von Pyrazolonen unter Verwendung aliphatischer und aromatischer Hydrazine sowie Hydrazin-Hydrochloride untersucht. Es wurden 9 verschiedene Pyrazolone in Gesamtausbeuten von 45 - $96 \%$ erhalten. Damit konnte gezeigt werden, daß bei der von L. F. Tietze und A. Steinmetz entwickelten Festphasen-Pyrazolonsynthesen unter Verwendung von Hydrazin eine wesentliche Steigerung der Diversität durch Einsatz von substituierten Hydrazinen möglich ist.

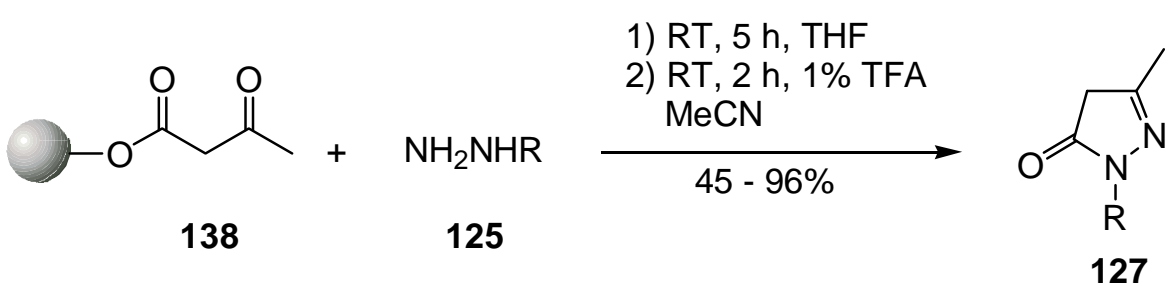

\begin{tabular}{|c|c|c|c|}
\hline Hydrazin & $\mathrm{R}$ & Pyrazolon & Ausbeute (\%) \\
\hline $\mathbf{3 1 1}$ & $4-\mathrm{CF}_{3}-\mathrm{Ph}$ & $\mathbf{3 1 6}$ & 94 \\
\hline $\mathbf{3 1 2}$ & $4-\mathrm{SO}_{2} \mathrm{Me}-\mathrm{Ph}$ & $\mathbf{3 1 7}$ & 96 \\
\hline $\mathbf{3 1 3}$ & $4-\mathrm{NO}_{2}-\mathrm{Ph}$ & $\mathbf{3 1 8}$ & 55 \\
\hline $\mathbf{3 1 4}$ & $\mathrm{C}_{7} \mathrm{H}_{6} \mathrm{ClN}_{2}$ & $\mathbf{3 1 9}$ & 54 \\
\hline $\mathbf{3 1 5}$ & $\mathrm{CH}_{2} \mathrm{CF}_{3}$ & $\mathbf{3 2 0}$ & 49 \\
\hline $\mathbf{3 2 1}^{32}$ & $2-\mathrm{NO}_{2}-\mathrm{Ph}$ & $\mathbf{3 2 3}$ & $57^{\mathrm{a}}$ \\
\hline $\mathbf{3 2 4}^{\mathrm{b}}$ & $2,4-\mathrm{F}_{2}-\mathrm{Ph}$ & $\mathbf{3 2 7}$ & 50 \\
\hline $\mathbf{3 2 5}^{\mathrm{b}}$ & $(1-\mathrm{Ph})-\mathrm{Et}$ & $\mathbf{3 2 8}$ & 63 \\
\hline $\mathbf{3 2 6}^{\mathrm{b}}$ & $(1-\mathrm{Me})-c y c l-\mathrm{Hex}$ & $\mathbf{3 2 9}$ & 45 \\
\hline
\end{tabular}

\footnotetext{
${ }^{\mathrm{a}}$ Die cyclisierende Abspaltung erfolgte bei $80^{\circ} \mathrm{C}$ in Toluol.

${ }^{\mathrm{b}}$ Hydrochlorid.
}

Tabelle 16: Synthetisierte Pyrazolone. 
In der vorliegenden Arbeit gelang es, die allylische Alkylierung an der festen Phase zu etablieren und damit das Methodenrepertoire für Synthesen am polymeren Träger zu erweitern. Ferner wurde die Synthesesequenz von Pyrazolonen an der festen Phase durch die Variation verschiedener Hydrazine komplettiert. Beide Transformationen stehen nun zur Durchführung von kombinatorischen Synthesen zum Aufbau von Substanzbibliotheken zur Verfügung. 


\section{B. Experimenteller Teil}

\section{Allgemeine Methoden}

Alle Umsetzungen wurden, soweit nötig, in ausgeheizten Glasapparaturen unter einem leichten Argon-Überdruck durchgeführt. Palladium-katalysierte Reaktionen wurden in entgasten Solventien durchgeführt. Die Lösungsmittel wurden entsprechend den üblichen Labormethoden getrocknet und destilliert. Käufliche Edukte wurden ohne weitere Reinigung eingesetzt, sofern nicht anders angegeben.

\subsection{Verwendete Geräte}

\section{Schmelzpunkte:}

Schmelzpunktbestimmungsapparatur FP61 der Firma Mettler. Die Werte sind nicht korrigiert.

\section{Infrarotspektren:}

Modell IFS 25 und Vektor 22 der Firma Bruker. Kristalline Substanzen wurden als $\mathrm{KBr}$ Preßlinge, nichtkristalline Verbindungen als Film zwischen KBr-Platten gemessen. Zur Kalibrierung diente eine Polystyrolbande bei $1601 \mathrm{~cm}^{-1}$.

\section{UV/VIS-Spektren:}

Modelle Lambda 2 und Lambda 9 der Firma Perkin-Elmer.

\section{${ }^{1}$ H-NMR-Spektren:}

Modelle XL-200 (200 MHz), VXR-200 (200 MHz) und VXR-500 (500 MHz) der Firma Varian, Modell AMX-300 (300 MHz) der Firma Bruker. Die chemischen Verschiebungen sind in Einheiten der $\delta$-Skala angegeben, Tetramethylsilan $\left(\delta_{\mathrm{TMS}}=0.00 \quad \mathrm{ppm}\right), \quad$ Benzol $\left(\delta_{\mathrm{TMS}}=7.26 \mathrm{ppm}\right)$ und Chloroform $\left(\delta_{\mathrm{TMS}}=7.24 \mathrm{ppm}\right)$ dienten als interner Standard. Zur Kennzeichnung der Multiplizitäten der Signale werden folgende Abkürzungen verwendet: $\mathrm{s}$ (Singulett), d (Dublett), $\mathrm{t}$ (Triplett), q (Quartett), quint (Quintett), m (Multiplett), $\mathrm{m}_{\mathrm{c}}$ (zentriertes Multiplett), br. (breit). Die Kopplungkonstanten $J$ sind in Hertz (Hz) angegeben. 


\section{${ }^{13}$ C-NMR-Spektren:}

Modell XL-200 (50.3 MHz), VXR-200 (50.3 MHz) und VXR-500 (125.7 MHz) der Firma Varian, Modell AMX-300 (75.5 MHz) der Firma Bruker. Die chemischen Verschiebungen ( $\delta$-Skala) sind den ${ }^{1} \mathrm{H}$-breitbandentkoppelten Spektren entnommen, die Multiplizitäten der Signale wurden in multiple-selection-Experimenten (APT-Pulsfolge) bestimmt. Als interner

Standard dienten die Signale von Tetramethylsilan $\left(\delta_{\mathrm{TMS}}=0.00 \mathrm{ppm}\right)$ und Benzol $\left(\delta_{\mathrm{TMS}}=128.50 \mathrm{ppm}\right)$.

\section{Massenspektren:}

Modelle MAT 95 der Firma Finnigan. In Klammern sind die relativen Intensitäten bezogen auf den Basispeak $(\mathrm{I}=100)$ angegeben.

\section{Elementaranalysen:}

Mikroanalytisches Labor des Institutes für Organische Chemie der Universität Göttingen.

\subsection{Chromatographische Methoden}

\section{Dünnschichtchromatographie (DC):}

Es wurden DC-Fertigfolien SIL G/UV 254 der Firma Macherey, Nagel \& Co., (Schichtstärke $0.25 \mathrm{~mm}$ ) verwendet. Angegeben sind $\mathrm{R}_{\mathrm{f}}$-Werte (Laufhöhe relativ zur Laufmittelfront). Neben der UV-Detektion dienten eine 1\%ige Kaliumpermanganatlösung, eine Vanilin-SchwefelsäureLösung (425 ml Methanol, $50 \mathrm{ml}$ Eisessig, $15 \mathrm{ml}$ konz. Schwefelsäure, $2.5 \mathrm{~g}$ Vanillin) sowie eine Molybdatophosphorsäure-Lösung als Anfärbereagenzien.

\section{Säulenfiltration (SF) und Säulenchromatographie (SC):}

Für die Arbeiten unter normalem Druck wurde Kieselgel der Korngröße 0.063 - 0.200 mm der Firma Macherey, Nagel \& Co. verwendet, für die Arbeiten unter erhöhtem Druck (Flash Chromatographie, FC) Kieselgel derselben Firma mit 0.032 - 0.063 mm Korngröße.

\subsection{Allgemeine Bemerkungen zu Festphasenreaktionen}

Die beschriebenen Reaktionen an fester Phase wurden in der geringstmöglichen Menge an Lösungsmittel durchgeführt. Es wurde jeweils nur soviel Solvens verwendet, daß das Harz vollständig gequollen und die Rührbarkeit der Suspension gewährleistet war. Es wurden Über- 
schüsse an Reagenzien und im Vergleich zu Reaktionen in Lösung verlängerte Reaktionszeiten verwendet, um vollständige Umsätze zu ermöglichen.

Aufgrund der eingeschränkten Möglichkeiten zur Charakterisierung polymergebundener Verbindungen ${ }^{[5]}$ konnten nur die abgespaltenen Zielstrukturen spektroskopisch vollständig identifiziert werden. Einige Transformationen an der festen Phase konnten durch Aufnahme von FTIR-Spektren verfolgt werden. Da im Rahmen der Kombinatorischen Chemie jeweils nur geringste Mengen an Produkten erhalten werden, wurde auf Verbrennungsanalysen verzichtet und i. a. hochauflösende Massenspektren angefertigt.

Alle angegebenen Ausbeuten sind als Gesamtausbeuten über mehrere Stufen zu verstehen. Sie sind bezogen auf eine Beladungsdichte von 0.54 - $0.75 \mathrm{mmol} \mathrm{OH/g}$ (Kapitel 5.1.) des spacermodifizierten Merrifield-Harzes 6 bzw. 1 mmol OH/g des Wang-Harzes 3. 


\section{Allgemeine Arbeitsvorschriften}

\subsection{Synthesen in flüssiger Phase}

\subsubsection{AAV 1: DIBAH-Reduktion von Enonen}

Das Enon (30 mmol, 1 eq.) wird in trockenem $\mathrm{CH}_{2} \mathrm{Cl}_{2}(60 \mathrm{ml})$ gelöst und die Mischung auf $-78^{\circ} \mathrm{C}$ abgekühlt. Man gibt tropfenweise eine $1 \mathrm{M}$ Lösung von DIBAH in Hexan (1.1 eq.) hinzu und läßt $1 \mathrm{~h}$ bei $-78^{\circ} \mathrm{C}$ rühren. Nach dem Erwärmen auf $0{ }^{\circ} \mathrm{C}$ wird mit ges. $\mathrm{NH}_{4}$ Cl-Lösung $(5 \mathrm{ml})$ versetzt, über Celite filtriert und gründlich mit $\mathrm{CH}_{2} \mathrm{Cl}_{2}$ (100 ml) gewaschen. Die abgetrennte wäßrige Phase extrahiert man dreimal mit $\mathrm{Et}_{2} \mathrm{O}(10 \mathrm{ml})$, wäscht mit ges. NaCl-Lösung $(5 \mathrm{ml})$, trocknet über $\mathrm{Na}_{2} \mathrm{SO}_{4}$ und entfernt das Lösungsmittel im Vakuum. Das erhaltene Rohprodukt wird ohne weitere Reinigung in der anschließenden Acetylierung eingesetzt.

\subsubsection{AAV 2: Grignard-Reaktion aromatischer Aldehyde}

Der Aldehyd (10 mmol, 1 eq.) wird in trockenem THF (25 ml) vorgelegt und auf $0{ }^{\circ} \mathrm{C}$ gekühlt. Nach sehr langsamer Zugabe von Vinyl-Magnesium-Bromid (1.5 eq.) als 1 M Lösung in THF wird auf Raumtemperatur erwärmt und $2 \mathrm{~h}$ gerührt. Bei $0{ }^{\circ} \mathrm{C}$ versetzt man mit ges. $\mathrm{NH}_{4} \mathrm{Cl}-$ Lösung $(10 \mathrm{ml})$ und extrahiert die wäßrige Phase dreimal mit $\mathrm{Et}_{2} \mathrm{O}(10 \mathrm{ml})$. Die vereinigten organischen Phasen werden mit ges. $\mathrm{NaHCO}_{3}$-Lösung $(10 \mathrm{ml})$ und ges. NaCl-Lösung $(10 \mathrm{ml})$ gewaschen, über $\mathrm{Na}_{2} \mathrm{SO}_{4}$ getrocknet und im Vakuum eingeengt. Das erhaltene Rohprodukt wird ohne weitere Reinigung in der anschließenden Acetylierung eingesetzt.

\subsubsection{AAV 3: Acetylierung von Allylalkoholen}

Der Allylalkohol (10 mmol, 1 eq.) wird in trockenem $\mathrm{CH}_{2} \mathrm{Cl}_{2}(20 \mathrm{ml})$ vorgelegt, mit $\mathrm{NEt}_{3}$ (1.1 eq.) und DMAP (0.05 eq.) versetzt und auf $0{ }^{\circ} \mathrm{C}$ gekühlt. Nach der tropfenweisen Zugabe von $\mathrm{Ac}_{2} \mathrm{O}$ (1.1 eq.) rührt man $1 \mathrm{~h}$ bei $0{ }^{\circ} \mathrm{C}$. Man fügt $\mathrm{H}_{2} \mathrm{O}(5 \mathrm{ml})$ hinzu, trennt die Phasen und extrahiert die wäßrige Phase mit $\mathrm{Et}_{2} \mathrm{O}$ (dreimal $10 \mathrm{ml}$ ). Die vereinigten organischen Phasen werden mit ges. $\mathrm{NaHCO}_{3}$-Lösung $(10 \mathrm{ml}), 0.5 \mathrm{M} \mathrm{HCl}$-Lösung $(10 \mathrm{ml})$ und ges. NaCl-Lösung 
$(10 \mathrm{ml})$ gewaschen und über $\mathrm{Na}_{2} \mathrm{SO}_{4}$ getrocknet. Nach dem Einengen im Vakuum wird das Rohprodukt i. a. ohne weitere Reinigung in die folgende Reaktion eingesetzt.

\subsubsection{AAV 4: Darstellung von Carbonaten aus Allylalkoholen}

Der Allylalkohol (10 mmol, 1 eq.) wird in trockenem $\mathrm{CH}_{2} \mathrm{Cl}_{2}(20 \mathrm{ml})$ vorgelegt, mit $\mathrm{NEt}_{3}$ (1.1 eq.) und DMAP (0.05 eq.) versetzt und auf $0{ }^{\circ} \mathrm{C}$ gekühlt. Nach der tropfenweisen Zugabe von Chlorameisensäureethylester (1.1 eq.) rührt man $1 \mathrm{~h}$ bei $0{ }^{\circ} \mathrm{C}$ und dann $2 \mathrm{~h}$ bei Raumtemperatur. Man fügt $\mathrm{H}_{2} \mathrm{O}(5 \mathrm{ml})$ hinzu, trennt die Phasen und extrahiert die wäßrige Phase mit $\mathrm{Et}_{2} \mathrm{O}$ (dreimal $10 \mathrm{ml}$ ). Die vereinigten organischen Phasen werden mit ges. $\mathrm{NaHCO}_{3}$ - $\mathrm{Lösung}$ $(10 \mathrm{ml}), 0.5 \mathrm{M} \mathrm{HCl}$-Lösung $(10 \mathrm{ml})$ und ges. NaCl-Lösung $(10 \mathrm{ml})$ gewaschen und über $\mathrm{Na}_{2} \mathrm{SO}_{4}$ getrocknet. Nach dem Einengen im Vakuum wird das Rohprodukt säulenchromatographisch an Kieselgel gereinigt.

\subsubsection{AAV 5: Allylische Alkylierung von 1,3-Dicarbonylverbindungen}

Die 1,3-Dicarbonylverbindung (1.0 mmol, 1 eq.) wird in entgastem THF (2 ml) gelöst. Anschließend fügt man die Allylkomponente (2.1 mmol, 2.1 eq.), N,O-Bis(trimethylsilyl)acetamid (1.0 mmol, 2 eq.), Tetrakis(triphenylphosphan)palladium (10 mol\%) und eine Spatelspitze KOAc hinzu und rührt für die angegebene Zeit bei der angegebenen Temperatur. Nach beendeter Reaktion wird über Kieselgel säulenfiltriert und das Rohprodukt säulenchromatographisch an Kieselgel gereinigt.

\subsection{Synthesen am polymeren Träger}

\subsubsection{AAV 6: Allylische Alkylierung von 1,3-Dicarbonylverbindungen mit Allylacetaten}

Die polymergebundene 1,3-Dicarbonylverbindung (300 mg, 1 eq.) wird in trockenem THF (4 ml) gequollen und die Suspension entgast, indem $1 \mathrm{~h}$ lang Argon durch die Reaktionsmischung geleitet wird. Man fügt das Allylacetat (5 eq.), N,O-Bis(trimethylsilyl)acetamid (5 eq.), Tetrakis(triphenylphosphan)palladium $(20 \mathrm{~mol} \%)$ und eine Spatelspitze KOAchinzu. Man rührt für die angegebene Zeit bei der angegebenen Temperatur. Anschließend wird abfiltriert, das Harz mit THF (20 ml), DMF (20 ml), THF (20 ml), MeOH (20 ml) und $\mathrm{CH}_{2} \mathrm{Cl}_{2}(20 \mathrm{ml})$ gewaschen und bei $55^{\circ} \mathrm{C}$ im Vakuum getrocknet. 


\subsubsection{AAV 7: Allylische Alkylierung von 1,3-Dicarbonylverbindungen mit Allylcarbo- naten}

Die polymergebundene 1,3-Dicarbonylverbindung (300 mg, 1 eq.) wird vorgelegt und durch mehrfaches Evakuieren und Belüften des Kolbens mit Argon entgast. Anschließend wird das Harz in trockenem, entgastem THF (4 ml) gequollen. Man fügt das Allylcarbonat (5 eq.) und Tetrakis(triphenylphosphan)palladium (20 mol\%) hinzu und rührt für die angegebene Zeit bei der angegebenen Temperatur. Anschließend wird abfiltriert, das Harz mit THF (20 ml), DMF (20 ml), THF (20 ml), MeOH $(20 \mathrm{ml})$ und $\mathrm{CH}_{2} \mathrm{Cl}_{2}(20 \mathrm{ml})$ gewaschen und bei $55^{\circ} \mathrm{C}$ im Vakuum getrocknet.

\subsubsection{AAV 8: Reduktive Abspaltung polymergebundener 1,3-Dicarbonylverbindungen}

Die in trockenem Toluol $(5 \mathrm{ml})$ gequollene polymergebundene 1,3-Dicarbonylverbindung (300 mg) wird bei $0{ }^{\circ} \mathrm{C}$ mit $1 \mathrm{M}$ DIBAH-Lösung in Hexan (10 eq.) versetzt und 5 Stunden bei gleichbleibender Temperatur gerührt. Nach beendeter Reaktion zerstört man überschüssiges Reagenz durch vorsichtige Zugabe von $\mathrm{MeOH}(0.5 \mathrm{ml})$ bei $0{ }^{\circ} \mathrm{C}$ und überführt die gesamte Reaktionsmischung einschließlich des Harzes in einen Scheidetrichter. Die Mischung wird mit ges. Natrium-Kalium-Tartrat-Lösung $(5 \mathrm{ml})$ gewaschen und die wäßrige Phase solange mit $\mathrm{Et}_{2} \mathrm{O}(5 \mathrm{ml})$ extrahiert, bis sich dünnschichtchromatographisch kein Produkt mehr nachweisen läßt. Die vereinigten organischen Phasen wäscht man mit ges. NaCl-Lösung, trocknet über $\mathrm{Na}_{2} \mathrm{SO}_{4}$ und engt im Vakuum ein. Das Rohprodukt wird säulenchromatographisch gereinigt.

\subsubsection{AAV 9: Abspaltung von polymergebundenen 1,3-Dicarbonylverbindungen durch basische Umesterung}

Die in trockenem THF (3 ml) gequollene polymergebundene 1,3-Dicarbonylverbindung (300 mg) wird mit 5.4 M NaOMe-Lösung in $\mathrm{MeOH}$ (3 eq.) versetzt und 3 Stunden bei Raumtemperatur gerührt. Anschließend wird das Harz filtriert und mit $\mathrm{MeOH}$ (20 ml) gewaschen, wobei die organische Phase direkt auf sauren Ionentauscher Amberlite IR 120 (2 g) gegeben wird. Nach zweistündigem Rühren wird filtriert, mit $\mathrm{MeOH}$ gewaschen und die organische Phase im Vakuum eingeengt. 


\subsubsection{AAV 10: Synthese von Pyrazolonen}

Das in trockenem THF (3 ml) gequollene polymergebundene Acetoacetat $(300 \mathrm{mg}$ ) wird mit dem Hydrazin (10 eq.) versetzt und 5 Stunden bei Raumtemperatur gerührt. Anschließend wird abfiltriert und das Harz mit THF (20ml), $\mathrm{MeOH}(20 \mathrm{ml}), \mathrm{CH}_{2} \mathrm{Cl}_{2}$ (20ml) und $\mathrm{MeCN}(20 \mathrm{ml})$ gewaschen.

Der polymere Träger wird nicht getrocknet, sondern im MeCN gequollenen Zustand in einen ausgeheizten Kolben überführt und mit einer 1\%igen Lösung von Trifluoressigsäure in MeCN versetzt. Nach zweistündigem Rühren bei Raumtemperatur filtriert man ab, wäscht mit $\mathrm{MeCN}$ $(20 \mathrm{ml})$ und Toluol $(20 \mathrm{ml})$ und engt im Vakuum ein. Zur vollständigen Entfernung der Trifluoressigsäure wird zweimal Toluol $(20 \mathrm{ml})$ zugegeben und im Vakuum eingeengt. Das Produkt wird im Ölpumpenvakuum getrocknet. 


\section{Synthesen in flüssiger Phase}

\subsection{Synthese der Allylacetate}

\subsection{1. (E/Z)-Essigsäure-but-2-enylester 156}

Gemäß AAV 3 wurde (E/Z)-2-Buten-1-ol 149 (1.70 ml, 1.44 g, 20.0 mmol) acetyliert. Man erhielt $1.47 \mathrm{~g}$ (12.8 mmol, 64\%) einer farblosen Flüssigkeit.

$\mathbf{R}_{\mathbf{f}}=0.50($ Pentan : Essigester $=10: 1)$.

${ }^{1}$ H-NMR $\left(200 \mathrm{MHz}, \mathrm{CDCl}_{3}\right): \delta=1.73\left(\mathrm{~d}, J=6.5 \mathrm{~Hz}, 3 \mathrm{H}, 4^{\prime}-\mathrm{H}_{3}\right), 2.06$ (s, $\left.3 \mathrm{H}, 2-\mathrm{H}_{3}\right), 4.50$ (d, $\left.J=6.5 \mathrm{~Hz}, 2 \mathrm{H}, 1^{\prime}-\mathrm{H}_{2}\right), 5.50-5.91$ (m, $\left.2 \mathrm{H}, 2^{\prime}-\mathrm{H}, 3^{\prime}-\mathrm{H}\right)$.

${ }^{13}$ C-NMR (50.3 MHz, $\mathrm{CDCl}_{3}$ ): $\delta=17.80$ (C-4'), 21.03 (C-2), 65.25 (C-1'), 125.0 (C-3'), $131.4(\mathrm{C}-2$ ') $), 170.8$ (C-1).

MS $(70 \mathrm{eV}, \mathrm{EI}): \mathrm{m} / \mathrm{z}(\%)=114(13)\left[\mathrm{M}^{+}\right], 71(28)\left[\mathrm{M}^{+}-\mathrm{CH}_{3} \mathrm{O}\right], 43(100)\left[\mathrm{CH}_{3} \mathrm{O}^{+}\right]$.

$\mathrm{C}_{5} \mathrm{H}_{8} \mathrm{O}_{2}(\mathbf{1 0 0 . 1 1})$

\subsubsection{Essigsäure-1-methylallylester 157}

Gemäß AAV 3 wurde 1-Buten-3-ol 150 (5.24 ml, 4.32 g, 60.0 mmol) acetyliert. Man erhielt $5.54 \mathrm{~g}(48.6 \mathrm{mmol}, 93 \%)$ einer leicht gelblichen Flüssigkeit.

$\mathbf{R}_{\mathbf{f}}=0.55($ Pentan : Essigester $=10: 1)$.

${ }^{1} \mathbf{H}$-NMR $\left(200 \mathrm{MHz}, \mathrm{CDCl}_{3}\right): \delta=1.32\left(\mathrm{~d}, J=6.2 \mathrm{~Hz}, 3 \mathrm{H}, 1\right.$ ' $\left.-\mathrm{CH}_{3}\right), 2.06$ (s, $\left.3 \mathrm{H}, 2-\mathrm{H}_{3}\right), 4.14$ $\left(\mathrm{d}, J=10.5 \mathrm{~Hz}, 2 \mathrm{H}, 3^{\prime}-\mathrm{H}_{\mathrm{a}}\right), 5.24\left(\mathrm{~d}, J=16.4 \mathrm{~Hz}, 1 \mathrm{H}, 3\right.$ '- $\left.\mathrm{H}_{\mathrm{b}}\right), 5.29-5.42$ (dq, $J=6.0,5.9 \mathrm{~Hz}$, $1 \mathrm{H}, 1$ '-H), 5.84 (ddd, $J=16.4,10.5,5.9 \mathrm{~Hz}, 1 \mathrm{H}, 2^{\prime}$-H).

${ }^{13}$ C-NMR (50.3 MHz, $\mathrm{CDCl}_{3}$ ): $\delta=19.90$ (1'-C), 21.31 (C-2), 70.99 (C-1'), 115.7 (C-3'), $137.6(\mathrm{C}-2$ ') $), 170.2(\mathrm{C}-1)$.

MS $(70 \mathrm{eV}, \mathrm{EI}): \mathrm{m} / \mathrm{z}(\%)=114(5)\left[\mathrm{M}^{+}\right], 72(70)\left[\mathrm{M}^{+}-\mathrm{CH}_{3} \mathrm{O}+\mathrm{H}\right], 43(100)\left[\mathrm{CH}_{3} \mathrm{O}^{+}\right]$.

$\mathrm{C}_{6} \mathrm{H}_{10} \mathrm{O}_{2}(\mathbf{1 1 4 . 1 4})$ 


\subsubsection{Essigsäure-2-methylallylester 158}

Gemäß AAV 3 wurde 2-Methyl-2-propen-1-ol 151 (4.24 ml, 3.60 g, 50.0 mmol) acetyliert. Es wurden $4.05 \mathrm{~g}$ (35.5 mmol, 71\%) der Zielverbindung als klare Flüssigkeit erhalten.

$\mathbf{R}_{\mathbf{f}}=0.50($ Pentan : Essigester $=10: 1)$.

${ }^{1} \mathbf{H}-\mathrm{NMR}\left(200 \mathrm{MHz}, \mathrm{CDCl}_{3}\right): \delta=1.76$ (s, $3 \mathrm{H}, 2$ '- $\mathrm{CH}_{3}$ ), 2.10 (s, $3 \mathrm{H}, 2-\mathrm{H}_{3}$ ), 4.50 (s, $2 \mathrm{H}, 3$ '$\left.\mathrm{H}_{2}\right), 4.93$ (s, $1 \mathrm{H}, 1^{\prime}-\mathrm{H}_{\mathrm{a}}$ ), 4.97 (s, $\left.1 \mathrm{H}, 1^{\prime}-\mathrm{H}_{\mathrm{b}}\right)$.

${ }^{13}$ C-NMR (50.3 MHz, $\mathrm{CDCl}_{3}$ ): $\delta=19.50$ (2'-C), 20.90 (C-2), 67.75 (C-1'), 112.8 (C-3'), 139.9 (C-2'), 170.7 (C-1).

MS $(70 \mathrm{eV}, \mathrm{EI}): \mathrm{m} / \mathrm{z}(\%)=114(8)\left[\mathrm{M}^{+}\right], 72(60)\left[\mathrm{M}^{+}-\mathrm{CH}_{3} \mathrm{O}+\mathrm{H}\right], 43(100)\left[\mathrm{CH}_{3} \mathrm{O}^{+}\right]$.

$\mathrm{C}_{6} \mathrm{H}_{10} \mathrm{O}_{2}(\mathbf{1 1 4 . 1 4})$

\subsection{4. (E)-Essigsäure-5-(2,5-dimethoxy-3,4,6-trimethylphenyl)-3-methyl-pent -2-enylester 159}

Gemäß AAV 3 wurde (E)-5-(2,5-Dimethoxy-3,4,6-trimethylphenyl)-3-methyl-pent-2-en-1-ol 152 (0.97 g, $3.5 \mathrm{mmol})$ acetyliert. Man erhielt $1.11 \mathrm{~g}$ (12.8 mmol, 99\%) eines weißen Feststoffes.

$\mathbf{R}_{\mathbf{f}}=0.55($ Pentan : Essigester $=10: 1)$.

${ }^{1}$ H-NMR $\left(200 \mathrm{MHz}, \mathrm{CDCl}_{3}\right): \delta=1.81$ (s, $3 \mathrm{H}, 3$ ' - $\mathrm{CH}_{3}$ ), 2.07 (s, $3 \mathrm{H}, 2-\mathrm{H}_{3}$ ), 2.18 (s, $9 \mathrm{H}, \mathrm{Ph}-$ $\mathrm{CH}_{3}$ ), 2.22 (t, $\left.J=7.9 \mathrm{~Hz}, 2 \mathrm{H}, 4^{\prime}-\mathrm{H}_{2}\right), 2.72$ (t, $\left.J=7.9 \mathrm{~Hz}, 2 \mathrm{H}, 5^{\prime}-\mathrm{H}_{2}\right), 3.64$ (s, $\left.3 \mathrm{H}, \mathrm{OMe}\right)$, 3.68 (s, $3 \mathrm{H}, \mathrm{OMe}), 4.63$ (d, $\left.J=7.0 \mathrm{~Hz}, 2 \mathrm{H}, 1^{\prime}-\mathrm{H}_{2}\right), 5.43$ (t, $\left.J=7.0 \mathrm{~Hz}, 1 \mathrm{H}, 2^{\prime}-\mathrm{H}\right)$.

${ }^{13} \mathbf{C}-N M R\left(50.3 \mathrm{MHz}, \mathrm{CDCl}_{3}\right): \delta=12.03$ (9'-C), 12.71 (8'-C), 13.11 (11'-C), 16.56 (3'-C), 21.08 (C-2), 26.09 (C-5'), 39.91 (C-4'), 60.07, 60.96 (OMe), 62.95 (C-1'), 118.1 (C-2'), 127.1 (C-11'), 127.9 (C-8'), 128.3 (C-9'), 131.6 (C-6'), 142.4 (C-3'), 152.9 (C-7'), 153.0 (C$\left.10^{\prime}\right), 171.1(\mathrm{C}-1)$.

MS (DCI, $\left.\mathrm{NH}_{3}\right): \mathrm{m} / \mathrm{z}=338[\mathrm{M}+18]$.

$\mathrm{C}_{19} \mathrm{H}_{28} \mathrm{O}_{4}(320.42)$

\subsection{5. (E)-Essigsäure-3-phenylallylester 160}

Gemäß AAV 3 wurde (E)-Zimtalkohol 153 (2.68 g, 20.0 mmol) acetyliert. Es wurden $3.27 \mathrm{~g}$ (18.6 mmol, 93\%) einer leicht gelblichen Flüssigkeit erhalten. 
$\mathbf{R}_{\mathbf{f}}=0.55($ Pentan : Essigester $=10: 1)$.

${ }^{1}$ H-NMR $\left(200 \mathrm{MHz}, \mathrm{CDCl}_{3}\right): \delta=2.1\left(\mathrm{~s}, 3 \mathrm{H}, 2-\mathrm{H}_{3}\right), 4.73\left(\mathrm{dd}, J=6.4,1.3 \mathrm{~Hz}, 2 \mathrm{H}, 1\right.$ ' $\left.-\mathrm{H}_{2}\right)$, $6.28\left(\mathrm{dt}, J=16.1,6.4 \mathrm{~Hz}, 1 \mathrm{H}, 2^{\prime}-\mathrm{H}\right), 6.64$ (d, $\left.J=16.1 \mathrm{~Hz}, 1 \mathrm{H}, 3^{\prime}-\mathrm{H}\right), 7.25-7.43$ (m, $5 \mathrm{H}$, $\mathrm{Ph}-\mathrm{H})$.

${ }^{13}$ C-NMR (50.3 MHz, $\mathrm{CDCl}_{3}$ ): $\delta=20.97$ (C-2), 65.04 (C-1'), 123.1 (C-2'), 126.5 (C-5', C9'), 128.0 (C-7'), 128.5 (C-6', C-8'), 134.1 (C-3'), 136.6 (C-4'), 170.7 (C-1).

MS (DCI, $\left.\mathrm{NH}_{3}\right): \mathrm{m} / \mathrm{z}=194[\mathrm{M}+18]$.

$\mathrm{C}_{11} \mathrm{H}_{12} \mathrm{O}_{2}$ (176.21)

\subsection{6. (E)-Essigsäure-1-ethyl-3-phenylallylester 161}

Gemäß AAV 3 wurde (E)-1-Phenyl-pent-1-en-3-ol 154 (1.62 g, 10.0 mmol) acetyliert. Man erhielt $1.95 \mathrm{~g}$ (9.90 mmol, 99\%) einer klaren Flüssigkeit.

$\mathbf{R}_{\mathbf{f}}=0.75($ Pentan : Essigester $=10: 1)$.

${ }^{1}$ H-NMR $\left(200 \mathrm{MHz}_{\mathrm{f}} \mathrm{CDCl}_{3}\right): \delta=0.94\left(\mathrm{t}, J=7.8 \mathrm{~Hz}, 3 \mathrm{H}, 2\right.$ ''- $\left.-\mathrm{H}_{3}\right), 1.65-1.84$ (m, $2 \mathrm{H}, 1$ ''$\left.\mathrm{H}_{2}\right), 2.08\left(\mathrm{~s}, 3 \mathrm{H}, 2-\mathrm{H}_{3}\right), 5.34\left(\mathrm{q}, J=6.9 \mathrm{~Hz}, 1 \mathrm{H}, 1\right.$ '-H), 6.12 (dd, $J=16.0,6.9 \mathrm{~Hz}, 1 \mathrm{H}, 2^{\prime}-$ H), 6.60 (d, $J=16.0 \mathrm{~Hz}, 1 \mathrm{H}, 3$ '-H), 7.22-7.43 (m, $5 \mathrm{H}, \mathrm{Ph}-\mathrm{H})$.

${ }^{13}$ C-NMR (50.3 MHz, $\mathrm{CDCl}_{3}$ ): $\delta=9.57$ (C-2',), 21.31 (C-2), 27.59 (C1'’), 76.00 (C-1'), 126.5 (C-5', C-9'), 127.4 (C-2'), 127.7 (C-3'), 128.5 (C-6', C-8'), 132.5 (C-7'), 136.3 (C-4'), $170.4(\mathrm{C}-1)$.

MS $(70 \mathrm{eV}, \mathrm{EI}): \mathrm{m} / \mathrm{z}(\%)=204(25)\left[\mathrm{M}^{+}\right], 161(40)\left[\mathrm{M}^{+}-\mathrm{CH}_{3} \mathrm{O}\right], 133(100)\left[\mathrm{Ph}(\mathrm{CH})_{3} \mathrm{O}^{+}+\mathrm{H}\right]$, $77(20)\left[\mathrm{Ph}^{+}\right], 43(45)\left[\mathrm{CH}_{3} \mathrm{O}^{+}\right]$.

\section{$\mathrm{C}_{13} \mathrm{H}_{16} \mathrm{O}_{2}$ (204.26)}

\subsubsection{Essigsäure-cyclohex-2-enylester 175}

Gemäß AAV 1 wurde Cyclohex-2-enon 162 (2.90 ml, 2.88 g, 30.0 mmol) reduziert und gemäß AAV 3 acetyliert. Man erhielt $2.90 \mathrm{~g}(25.8 \mathrm{mmol}, 86 \%)$ einer klaren Flüssigkeit.

$\mathbf{R}_{\mathbf{f}}=0.70($ Pentan : Essigester $=10: 1)$.

${ }^{1}$ H-NMR $\left(200 \mathrm{MHz}, \mathrm{CDCl}_{3}\right): \delta=1.56-1.98\left(\mathrm{~m}, 6 \mathrm{H}, 4-\mathrm{H}_{2}, 5^{\prime}-\mathrm{H}_{2}, 6^{\prime}-\mathrm{H}_{2}\right), 2.02$ (s, $\left.3 \mathrm{H}, 2-\mathrm{H}_{3}\right)$, $5.72\left(\mathrm{~m}_{\mathrm{c}}, 1 \mathrm{H}, 3^{\prime}-\mathrm{H}\right)$, 5.63-5.77 (m, $\left.1 \mathrm{H}, 1^{\prime}-\mathrm{H}\right), 5.89-6.03$ (m, 1 H, 2'-H).

${ }^{13}$ C-NMR (50.3 MHz, $\left.\mathrm{CDCl}_{3}\right): \delta=18.90\left(\mathrm{C}^{2} 5^{\prime}\right), 21.37$ (C-2), 24.88 (C-4'), 28.32 (C-6'), 68.06 (C-1'), 125.7 (C-3'), 132.5 (C-2'), 170.6 (C-1). 
MS (70 eV, EI): m/z (\%) = $140(15)\left[\mathrm{M}^{+}\right], 98(65)\left[\mathrm{M}^{+}-\mathrm{CH}_{3} \mathrm{O}+\mathrm{H}\right], 79(100)\left[\mathrm{M}^{+}-\mathrm{CH}_{3} \mathrm{O}-\mathrm{H}_{2} \mathrm{O}\right]$, $43(90)\left[\mathrm{CH}_{3} \mathrm{O}^{+}\right]$.

$\mathrm{C}_{8} \mathrm{H}_{12} \mathrm{O}_{2}$ (140.17)

\subsubsection{Essigsäure-cyclopent-2-enylester 176}

Gemäß AAV 1 wurde Cyclopent-2-enon 163 (0.83 ml, 0.82 g, 10 mmol) reduziert und gemäß AAV 3 acetyliert. Man erhielt $0.98 \mathrm{~g}$ (7.8 mmol, 78\%) der Zielverbindung als klare Flüssigkeit.

$\mathbf{R}_{\mathbf{f}}=0.79($ Pentan : Essigester $=10: 1)$.

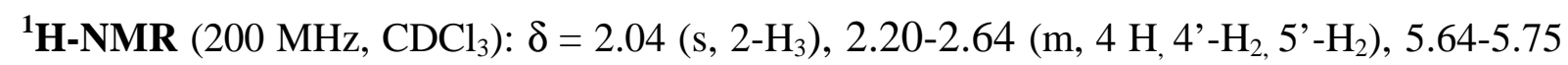
(m, 1 H, 3'-H), 5.79-5.87 (m, 1 H, 1'-H), 6.07-6.15 (m, 1 H, 2'-H).

${ }^{13}$ C-NMR (50.3 MHz, $\mathrm{CDCl}_{3}$ ): $\delta=21.31$ (C-2), 29.77 (C-4'), 31.06 (C-5'), 80.46 (C-1'), 129.2 (C-3'), 137.5 (C-2'), 171.0 (C-1).

MS $\left(\mathrm{DCI}, \mathrm{NH}_{3}\right): \mathrm{m} / \mathrm{z}=144[\mathrm{M}+18]$.

$\mathrm{C}_{7} \mathrm{H}_{10} \mathrm{O}_{2}$ (126.15)

\subsubsection{Essigsäure-2-methyl-cyclopent-2-enylester 177}

Gemäß AAV 1 wurde 2-Methyl-cyclopent-2-enon $164(0.78 \mathrm{ml}, 0.77 \mathrm{~g}, 8.0 \mathrm{mmol})$ reduziert und gemäß AAV 3 acetyliert. Es wurden 0.94 g (6.7 mmol, 84\%) einer klaren Flüssigkeit erhalten.

$\mathbf{R}_{\mathbf{f}}=0.77($ Pentan : Essigester $=10: 1)$.

${ }^{1} \mathbf{H}-\mathbf{N M R}\left(200 \mathrm{MHz}, \mathrm{CDCl}_{3}\right): \delta=1.64$ (s, $\left.3 \mathrm{H}, 2^{\prime}-\mathrm{CH}_{3}\right), 1.99$ (s, $\left.3 \mathrm{H}, 2-\mathrm{H}_{3}\right), 2.19-2.43$ (m, 4 $\left.\mathrm{H}, 4^{\prime}-\mathrm{H}_{2}, 5^{\prime}-\mathrm{H}_{2}\right)$.

${ }^{13}$ C-NMR (50.3 MHz, $\mathrm{CDCl}_{3}$ ): $\delta=13.81$ (2'-C), 21.30 (C-2), 30.24 (C-5'), 30.99 (C-4'), 82.37 (C-1'), 130.8 (C-3'), 138.0 (C-2'), 171.2 (C-1).

MS (70 eV, EI): m/z (\%) = $140(5)\left[\mathrm{M}^{+}\right], 97(55)\left[\mathrm{M}^{+}-\mathrm{CH}_{3} \mathrm{O}\right], 79(100)\left[\mathrm{M}^{+}-\mathrm{CH}_{3} \mathrm{O}-\mathrm{OH}\right], 43$ (50) $\left[\mathrm{CH}_{3} \mathrm{O}^{+}\right]$.

$\mathrm{C}_{8} \mathrm{H}_{12} \mathrm{O}_{2}$ (140.17) 


\subsubsection{Essigsäure-3-methyl-cyclopent-2-enylester 178}

Gemäß AAV 1 wurde 3-Methyl-cyclopent-2-enon 165 (0.78 ml, 0.77 g, 8.0 mmol) reduziert und gemäß AAV 3 acetyliert. Es wurden 1.04 g (7.3 mmol, 92\%) einer klaren Flüssigkeit erhalten.

$\mathbf{R}_{\mathbf{f}}=0.77($ Pentan : Essigester $=10: 1)$.

${ }^{1} \mathbf{H}-\mathbf{N M R}\left(200 \mathrm{MHz}, \mathrm{CDCl}_{3}\right): \delta=1.81\left(\mathrm{~s}, 3 \mathrm{H}, 3^{\prime}-\mathrm{CH}_{3}\right), 2.03$ (s, $\left.3 \mathrm{H}, 2-\mathrm{H}_{3}\right), 2.17-2.51$ (m, 4 $\left.\mathrm{H}, 4^{\prime}-\mathrm{H}_{2}, 5^{\prime}-\mathrm{H}_{2}\right), 5.45\left(\mathrm{~m}_{\mathrm{c}}, 1 \mathrm{H}, 1^{\prime}-\mathrm{H}\right), 5.65\left(\mathrm{~m}_{\mathrm{c}}, 1 \mathrm{H}, 2^{\prime}-\mathrm{H}\right)$.

${ }^{13}$ C-NMR (50.3 MHz, $\mathrm{CDCl}_{3}$ ): $\delta=16.79$ (3'-C), 21.37 (C-2), 30.79 (C-5'), 35.22 (C-4'), 81.37 (C-1'), 123.4 (C-2'), 148.5 (C-3'), 171.2 (C-1).

MS $\left(\mathrm{DCI}, \mathrm{NH}_{3}\right): \mathrm{m} / \mathrm{z}=158[\mathrm{M}+18]$.

$\mathrm{C}_{8} \mathrm{H}_{12} \mathrm{O}_{2}(\mathbf{1 4 0 . 1 7})$

\subsubsection{1. (E)-Essigsäure-3-(4-dimethylaminophenyl)-allylester 179}

Gemäß AAV 1 wurde (E)-4-Dimethylamino-zimtaldehyd 160 (1.75 g, 10.0 mmol) reduziert und gemäß AAV 3 acetyliert. Man erhielt $1.61 \mathrm{~g}$ (7.35mmol, 73\%) einer klaren Flüssigkeit.

$\mathbf{R}_{\mathbf{f}}=0.40($ Pentan : Essigester $=3: 1)$.

${ }^{1}$ H-NMR $\left(200 \mathrm{MHz}, \mathrm{CDCl}_{3}\right): \delta=2.09$ (s, $\left.3 \mathrm{H}, 2-\mathrm{H}_{3}\right), 2.97\left(\mathrm{~s}, 6 \mathrm{H}, \mathrm{NCH}_{3}\right), 4.69$ (d, $J=6.9$ $\mathrm{Hz}, 2 \mathrm{H}, 1$ ' $\left.-\mathrm{H}_{2}\right), 6.08$ (dt, $\left.J=15.6,6.9 \mathrm{~Hz}, 1 \mathrm{H}, 2^{\prime}-\mathrm{H}\right), 6.58$ (d, $\left.J=15.6 \mathrm{~Hz}, 1 \mathrm{H}, 3^{\prime}-\mathrm{H}\right), 6.68$ (d, $J=8.8 \mathrm{~Hz}, 2 \mathrm{H}, 3$ ' '-H, 5' '-H), 7.29 (d, J=8.8 Hz, $2 \mathrm{H}, 2$ ''-H, 6' '-H).

${ }^{13} \mathrm{C}-\mathrm{NMR}\left(50.3 \mathrm{MHz}, \mathrm{CDCl}_{3}\right): \delta=20.97(\mathrm{C}-2), 43.12\left(2 * \mathrm{NCH}_{3}\right), 65.04$ (C-1'), 123.1 (C-2'), 124.6 (C-4'), 126.5 (C-5', C-9'), 128.0 (C-7'), 128.5 (C-6', C-8'), 134.1 (C-3'), 170.7 (C-1). MS $\left(\mathrm{DCI}, \mathrm{NH}_{3}\right): \mathrm{m} / \mathrm{z}=237[\mathrm{M}+18]$.

$\mathrm{C}_{13} \mathrm{H}_{17} \mathrm{NO}_{2}$ (219.27)

\subsubsection{2. (E)-Essigsäure-3-(3-methoxyphenyl)-allylester 180}

(E)-3-Methoxyzimtsäure 167 (0.89 g, $5.0 \mathrm{mmol}, 1$ eq.) wurde in trockenem $\mathrm{CH}_{2} \mathrm{Cl}_{2}(20 \mathrm{ml})$ gelöst und die Mischung auf $-40{ }^{\circ} \mathrm{C}$ abgekühlt. Man gab tropfenweise eine $1 \mathrm{M}$ Lösung von DIBAH in Hexan (20 ml, $20 \mathrm{mmol}, 4$ eq.) hinzu, ließ auf Raumtemperatur kommen und rührte $3 \mathrm{~h}$ bei gleichbleibender Temperatur. Bei $0{ }^{\circ} \mathrm{C}$ wurde vorsichtig mit ges. $\mathrm{NH}_{4} \mathrm{Cl}$-Lösung $(5 \mathrm{ml})$ versetzt, über Celite filtriert und gründlich mit $\mathrm{CH}_{2} \mathrm{Cl}_{2}(100 \mathrm{ml})$ gewaschen. Die abgetrennte 
wäßrige Phase extrahierte man dreimal mit $\mathrm{Et}_{2} \mathrm{O}(10 \mathrm{ml})$, wusch die vereinigte organische Phase mit ges. NaCl-Lösung $(5 \mathrm{ml})$, trocknete über $\mathrm{Na}_{2} \mathrm{SO}_{4}$ und entfernte das Lösungsmittel im Vakuum. Das erhaltene Rohprodukt wurde gemäß AAV 3 acetyliert. Man erhielt $0.45 \mathrm{~g}$ (2.1 mmol, 42\%) eine klaren Flüssigkeit.

$\mathbf{R}_{\mathbf{f}}=0.50($ Pentan : Essigester $=10: 1)$.

${ }^{1} \mathbf{H}$-NMR $\left(200 \mathrm{MHz}, \mathrm{CDCl}_{3}\right): \delta=2.11\left(\mathrm{~s}, 3 \mathrm{H}, 2-\mathrm{H}_{3}\right), 3.82(\mathrm{~s}, 3 \mathrm{H}, \mathrm{OMe}), 4.73(\mathrm{~d}, J=6.3 \mathrm{~Hz}$, $2 \mathrm{H}, 1$ '- $\left.-\mathrm{H}_{2}\right), 6.28$ (dt, $\left.J=16.3,6.3 \mathrm{~Hz}, 1 \mathrm{H}, 2^{\prime}-\mathrm{H}\right), 6.64$ (d, $J=16.3 \mathrm{~Hz}, 1 \mathrm{H}, 3$ '-H), 6.82 (d, $J$ $\left.=7.8 \mathrm{~Hz}, 1 \mathrm{H}, 7^{\prime}-\mathrm{H}\right), 6.93\left(\mathrm{~s}, 1 \mathrm{H}, 5^{\prime}-\mathrm{H}\right), 6.99$ (d, $\left.J=7.8 \mathrm{~Hz}, 1 \mathrm{H}, 9^{\prime}-\mathrm{H}\right), 7.25$ (t, J = 7.8 Hz, $\left.1 \mathrm{H}, 8^{\prime}-\mathrm{H}\right)$.

${ }^{13}$ C-NMR (50.3 MHz, $\mathrm{CDCl}_{3}$ ): $\delta=20.97$ (C-2), 56.47 (OMe), 65.04 (C-1'), 111.5 (C-5'), 113.5 (C-7'), 118.1 (C-9'), 123.0 (C-2'), 129.0 (C-8'), 134.1 (C-3'), 136.1 (C-4'), 159.1 (C6'), 170.7 (C-1).

MS $\left(\mathrm{DCI}, \mathrm{NH}_{3}\right): \mathrm{m} / \mathrm{z}=224[\mathrm{M}+18]$.

$\mathrm{C}_{12} \mathrm{H}_{14} \mathrm{O}_{3}$ (206.23)

\subsubsection{Essigsäure-1-furan-3-yl-allylester 190}

Gemäß AAV 2 wurde Furan-3-carbaldehyd 181 (0.83 ml, 0.96 g, 10.0 mmol) umgesetzt und anschließend nach AAV 3 acetyliert. Man erhielt nach säulenchromatographischer Reinigung $(80 \mathrm{~g}$, Pentan : Essigester = $20: 1) 0.98 \mathrm{~g}(5.9 \mathrm{mmol}, 60 \%)$ der Zielverbindung als farblose Flüssigkeit.

$\mathbf{R}_{\mathbf{f}}=0.45($ Pentan : Essigester $=10: 1)$.

${ }^{1} \mathbf{H}-\mathbf{N M R}\left(200 \mathrm{MHz}, \mathrm{CDCl}_{3}\right): \delta=2.10\left(\mathrm{~s}, 3 \mathrm{H}, 2-\mathrm{H}_{3}\right), 5.28\left(\mathrm{~d}, J=10.8 \mathrm{~Hz}, 1 \mathrm{H}, 3^{\prime}-\mathrm{H}_{\mathrm{a}}\right), 5.34$ (d, $\left.J=16.7 \mathrm{~Hz}, 1 \mathrm{H}, 3^{\prime}-\mathrm{H}_{\mathrm{b}}\right), 6.01$ (ddd, $\left.J=16.7,10.8,6.0 \mathrm{~Hz}, 1 \mathrm{H}, 2^{\prime}-\mathrm{H}\right), 6.26$ (d, $J=6.0$ $\mathrm{Hz}, 1 \mathrm{H}, 1$ '-H), 6.38 ( $\mathrm{m}_{\mathrm{c}}, 1 \mathrm{H}, 5$ '’-H), 7.38-7.44 (m, 2 H, 2' '-H, 4' '-H).

${ }^{13}$ C-NMR (50.3 MHz, $\mathrm{CDCl}_{3}$ ): $\delta=21.11$ (C-2'’), 68.79 (C-1'), 109.2 (C-4), 117.2 (C-3'), 118.6 (C-3), 135.0 (C-2'), 140 (C-2), 143.2 (C-5), 169.8 (C=O).

MS $(70 \mathrm{eV}, \mathrm{EI}): \mathrm{m} / \mathrm{z}(\%)=166(15)\left[\mathrm{M}^{+}\right], 124(20)\left[\mathrm{M}^{+}-\mathrm{CH}_{3} \mathrm{O}+1\right], 67$ (15) $\left[\mathrm{C}_{4} \mathrm{H}_{4} \mathrm{O}^{+}\right], 43$ (100) $\left[\mathrm{CH}_{3} \mathrm{O}^{+}\right]$.

$\mathrm{C}_{9} \mathrm{H}_{10} \mathrm{O}_{2}$ (150.17) 


\subsubsection{Essigsäure-1-thiophen-2-yl-allylester 191}

Gemäß AAV 2 wurde Thiophen-2-carbaldehyd 182 (1.37 ml, 1.68 g, 15.0 mmol) umgesetzt und anschließend nach AAV 3 acetyliert. $1.34 \mathrm{~g}$ (7.41 mmol, 50\%) der Zielverbindung wurden nach säulenchromatographischer Reinigung $(80 \mathrm{~g}$, Pentan : Essigester $=20: 1)$ als leicht gelbliche Flüssigkeit erhalten.

$\mathbf{R}_{\mathbf{f}}=0.50($ Pentan : Essigester $=10: 1)$.

${ }^{1}$ H-NMR $\left(200 \mathrm{MHz}, \mathrm{CDCl}_{3}\right): \delta=2.10\left(\mathrm{~s}, 3 \mathrm{H}, 2-\mathrm{H}_{3}\right), 5.30\left(\mathrm{~d}, J=10.6 \mathrm{~Hz}, 1 \mathrm{H}, 3^{\prime}-\mathrm{H}_{\mathrm{a}}\right), 5.38$ (d, $J=16.1 \mathrm{~Hz}, 1 \mathrm{H}, 3^{\prime}-\mathrm{H}_{\mathrm{b}}$ ), 6.08 (ddd, $\left.J=16.1,10.6,6.1 \mathrm{~Hz}, 1 \mathrm{H}, 2^{\prime}-\mathrm{H}\right), 6.53$ (d, $J=6.1$ Hz, 1 H, 1'-H), 6.95-7.12 (m, 2 H, 3' '-H, 5' -H), 7.27-7.35 (m, 1 H, 4'”-H).

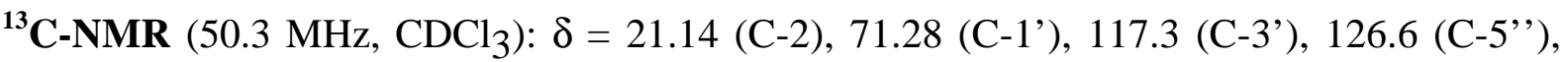
126.7 (C-3'’), 126.8 (C-4'’), 135.2 (C-2'), 141.7 (C-1'), 169.7 (C-1).

MS $\left(\mathrm{DCI}, \mathrm{NH}_{3}\right): \mathrm{m} / \mathrm{z}=200[\mathrm{M}+18]$.

$\mathrm{C}_{9} \mathrm{H}_{10} \mathrm{O}_{2} \mathrm{~S}(182.24)$

\subsubsection{Essigsäure-1-pyridin-4-yl-allylester 192}

Gemäß AAV 2 wurde Pyridin-4-carbaldehyd 183 (1.41 ml, 1.60 g, 15.0 mmol) umgesetzt und anschließend nach AAV 3 acetyliert. Es wurden nach säulenchromatographischer Reinigung (50 g, Pentan : Essigester = $3: 1) 0.55 \mathrm{~g}$ (3.12 mmol, 21\%) der gewünschten Verbindung als rötliches Öl erhalten.

$\mathbf{R}_{\mathbf{f}}=0.40($ Pentan : Essigester $=3: 1)$.

${ }^{1}$ H-NMR $\left(200 \mathrm{MHz}, \mathrm{CDCl}_{3}\right): \delta=2.15\left(\mathrm{~s}, 3 \mathrm{H}, 2\right.$ ' '- $\left.\mathrm{H}_{3}\right), 5.32$ (d, $\left.J=9.6 \mathrm{~Hz}, 1 \mathrm{H}, 3^{\prime}-\mathrm{H}_{\mathrm{a}}\right), 5.34$ $\left(\mathrm{d}, J=17.8 \mathrm{~Hz}, 1 \mathrm{H}, 3^{\prime}-\mathrm{H}_{\mathrm{b}}\right), 5.94$ (ddd, $\left.J=17.8,9.6,6.3 \mathrm{~Hz}, 1 \mathrm{H}, 2^{\prime}-\mathrm{H}\right), 6.21$ (d, $J=6.3 \mathrm{~Hz}$, $1 \mathrm{H}, 1$ '-H), 7.27 (d, J=4.1 Hz, $2 \mathrm{H}, 2$ ' '-H, 6' '-H), 8.60 (d, J = 4.1 Hz, 2 H, 3''-H, 5' '-H).

${ }^{13}$ C-NMR (50.3 MHz, $\mathrm{CDCl}_{3}$ ): $\delta=21.03$ (C-2), 80.28 (C-1'), 116.1 (C-3'), 129.6 (C-2', C6’'), 146.1 (C-1'’), 150.8 (C-3', C-5'), 135.2 (C-2'), 170.3 (C-1).

MS (DCI, $\left.\mathrm{NH}_{3}\right): \mathrm{m} / \mathrm{z}=195[\mathrm{M}+18]$.

$\mathrm{C}_{10} \mathrm{H}_{11} \mathrm{NO}_{2}$ (177.19) 


\subsubsection{Essigsäure-1-pyridin-2-yl-allylester 193}

Gemäß AAV 2 wurde Pyridin-2-carbaldehyd 184 (1.43 ml, 1.60 g, 15.0 mmol) umgesetzt und anschließend nach AAV 3 acetyliert. Nach säulenchromatographischer Reinigung (50 g, Pentan : Essigester = $5: 1)$ erhielt man $1.00 \mathrm{~g}$ (5.68 mmol, 38\%) der Zielverbindung als rötliches Öl erhalten.

$\mathbf{R}_{\mathbf{f}}=0.35$ (Pentan : Essigester $\left.=3: 1\right)$.

${ }^{1} \mathbf{H}-\mathbf{N M R}\left(200 \mathrm{MHz}, \mathrm{CDCl}_{3}\right): \delta=2.17\left(\mathrm{~s}, 3 \mathrm{H}, 2-\mathrm{H}_{3}\right), 5.31$ (d, $J=10.1 \mathrm{~Hz}, 1 \mathrm{H}, 3$ '- $\mathrm{H}_{\mathrm{a}}$ ), 5.39 $\left(\mathrm{d}, J=17.2 \mathrm{~Hz}, 1 \mathrm{H}, 3^{\prime}-\mathrm{H}_{\mathrm{b}}\right), 6.11\left(\mathrm{ddd}, J=17.2,10.1,6.7 \mathrm{~Hz}, 1 \mathrm{H}, 2^{\prime}-\mathrm{H}\right), 6.30$ (d, $J=6.7$ Hz, $\left.1 \mathrm{H}, 1^{\prime}-\mathrm{H}\right), 7.24$ (dt, J = 7.9, 1.8 Hz, 1 H, 4'-H), 7.36 (d, J = 7.9 Hz, $1 \mathrm{H}, 6$ ' -H), 7.70 (dt, $\left.J=7.9,1.8 \mathrm{~Hz}, 1 \mathrm{H}, 5^{\prime}-\mathrm{H}\right), 8.61$ (d, $\left.J=5.1 \mathrm{~Hz}, 1 \mathrm{H}, 3^{\prime}-\mathrm{H}\right)$.

${ }^{13}$ C-NMR (50.3 MHz, $\mathrm{CDCl}_{3}$ ): $\delta=21.15$ (C-2), 77.00 (C-1'), 117.8 (C-3'), 121.2 (C-4'’), 122.8 (C-6’'), 135.0 (C-2'), 136.8 (C-5'’), 149.4 (C-3’'), 158.0 (C-1'’), 169.8 (C-1).

MS $\left(\mathrm{DCI}, \mathrm{NH}_{3}\right): \mathrm{m} / \mathrm{z}=195[\mathrm{M}+18]$.

$\mathrm{C}_{10} \mathrm{H}_{11} \mathrm{NO}_{2}$ (177.19)

\subsubsection{7. (Z)-Essigsäure-4-acetoxy-but-2-enylester 272}

Gemäß AAV 3 wurde (Z)-But-2-en-1,4-ol 271 (1.64 ml, 1.76 g, 20.0 mmol) mit zwei Äquivalenten Essigsäureanhydrid acetyliert. Es wurden $3.37 \mathrm{~g}$ (19.6 mmol, 98\%) 272 als farblose Flüssigkeiten erhalten.

$\mathbf{R}_{\mathbf{f}}=0.80($ Pentan : Essigester $=3: 1)$.

${ }^{\mathbf{1}} \mathbf{H}-\mathrm{NMR}\left(200 \mathrm{MHz}, \mathrm{CDCl}_{3}\right): \delta=2.07\left(\mathrm{~s}, 6 \mathrm{H}, 2-\mathrm{H}_{3}, 2\right.$ ' ' $\left.-\mathrm{H}_{3}\right), 4.68\left(\mathrm{~d}, J=4.2 \mathrm{~Hz}, 4 \mathrm{H}, 1\right.$ ' $-\mathrm{H}_{2}$, 4'- $\left.\mathrm{H}_{2}\right), 5.75$ (t, $\left.J=4.2 \mathrm{~Hz}, 2 \mathrm{H}, 2^{\prime}-\mathrm{H}, 3^{\prime}-\mathrm{H}\right)$.

${ }^{13}$ C-NMR (50.3 MHz, CDCl $)$ ): $\delta=20.85$ (C-2, C-2'’), 60.37 (C-1', C-4'), 128.3 (C-2', C-3'), $171.3(\mathrm{C}=\mathrm{O})$.

MS $\left(\mathrm{DCI}, \mathrm{NH}_{3}\right): \mathrm{m} / \mathrm{z}=190[\mathrm{M}+18]$.

$\mathrm{C}_{8} \mathrm{H}_{12} \mathrm{O}_{4}$ (172.17)

\subsubsection{8. (Z)-Essigsäure-4-hydroxy-but-2-enylester 273 und ( $Z$ )-Essigsäure-4-acetoxy-} but-2-enylester 272

Gemäß AAV 3 wurde (Z)-But-2-en-1,4-ol 271 (3.28 ml, 3.52 g, 40.0 mmol) acetyliert, wobei das Essigsäureanhydrid sehr langsam zur Reaktionslösung hinzugegeben wurde. Nach säulen- 
chromatographischer Trennung an Kieselgel (60 g, Pentan : Essigester $=4: 1)$ wurden $2.33 \mathrm{~g}$ (17.9 mmol, 45\%) 273 und $1.85 \mathrm{~g}$ (10.7 mmol, 27\%) 272 als farblose Flüssigkeiten erhalten.

$\mathbf{R}_{\mathbf{f}}(\mathbf{2 7 3})=0.25($ Pentan : Essigester $=3: 1)$.

${ }^{1}$ H-NMR (200 MHz, $\mathrm{CDCl}_{3}$ ): $\delta=2.01$ (br. s, $\left.1 \mathrm{H}, \mathrm{OH}\right), 2.07$ (s, $\left.3 \mathrm{H}, 2-\mathrm{H}_{3}\right), 4.26$ (d, J = 6.4 $\left.\mathrm{Hz}, 2 \mathrm{H}, 4^{\prime}-\mathrm{H}_{2}\right), 4.68$ (d, $\left.J=6.4 \mathrm{~Hz}, 2 \mathrm{H}, 1^{\prime}-\mathrm{H}_{2}\right), 5.64$ (dtt, $J=11.2,6.8,1.0 \mathrm{~Hz}, 1 \mathrm{H}, 3$ '- $\mathrm{H}$ ), $5.86\left(\mathrm{dtt}, J=11.2,6.8,1.0 \mathrm{~Hz}, 1 \mathrm{H}, 2^{\prime}-\mathrm{H}\right)$.

${ }^{13}$ C-NMR (50.3 MHz, $\mathrm{CDCl}_{3}$ ): $\delta=20.99$ (C-2), 58.27 (C-4'), 60.23 (C-1'), 125.3 (C-3'), $133.4(\mathrm{C}-2$ ') $171.2(\mathrm{C}=\mathrm{O})$.

MS (DCI, $\left.\mathrm{NH}_{3}\right): \mathrm{m} / \mathrm{z}=148[\mathrm{M}+18]$.

$\mathrm{C}_{6} \mathrm{H}_{10} \mathrm{O}_{3}(\mathbf{1 3 0 . 1 4})$

Spektroskopische Daten der Verbindung 272 siehe 3.1.17.

\subsection{Synthese der Allylcarbonate}

\subsubsection{Allyl-ethyl-carbonat 196}

Gemäß AAV 4 wurde Allylalkohol $194(1.02 \mathrm{ml}, 0.87 \mathrm{~g}, 15.0 \mathrm{mmol})$ in die Zielverbindung umgewandelt. Nach säulenchromatographischer Reinigung an Kieselgel (5 g, Pentan : Essigester $=20: 1)$ wurden $1.38 \mathrm{~g}(10.6 \mathrm{mmol}, 71 \%)$ einer klaren Flüssigkeit erhalten.

$\mathbf{R}_{\mathbf{f}}=0.53($ Pentan : Essigester $=10: 1)$.

${ }^{1} \mathbf{H}$-NMR $\left(200 \mathrm{MHz}, \mathrm{CDCl}_{3}\right): \delta=1.32\left(\mathrm{t}, J=6.7 \mathrm{~Hz}, 3 \mathrm{H}, 2^{\prime}-\mathrm{H}_{3}\right), 4.21(\mathrm{q}, J=6.7 \mathrm{~Hz}, 2 \mathrm{H}$, $\left.1^{\prime}-\mathrm{H}_{2}\right), 4.64\left(\mathrm{~d}, J=5.6 \mathrm{~Hz}, 2 \mathrm{H}, 1-\mathrm{H}_{2}\right), 5.26\left(\mathrm{~d}, J=10.4 \mathrm{~Hz}, 1 \mathrm{H}, 3-\mathrm{H}_{\mathrm{a}}\right), 5.35(\mathrm{~d}, J=17.5$ $\mathrm{Hz}, 1 \mathrm{H}, 3-\mathrm{H}_{\mathrm{b}}$ ), 5.93 (ddt, $J=17.5,10.4,5.6 \mathrm{~Hz}, 1 \mathrm{H}, 2-\mathrm{H}$ ).

${ }^{13}$ C-NMR (50.3 MHz, $\mathrm{CDCl}_{3}$ ): $\delta=14.28$ (C-2'), 64.03 (C-1'), 68.25 (C-1), 118.7 (C-3), $131.6(\mathrm{C}-2), 154.9(\mathrm{C}=\mathrm{O})$.

MS (DCI, $\left.\mathrm{NH}_{3}\right): \mathrm{m} / \mathrm{z}=148[\mathrm{M}+18]$.

$\mathrm{C}_{6} \mathrm{H}_{10} \mathrm{O}_{3}(\mathbf{1 3 0 . 1 4})$ 


\subsection{2. (E)-3-Phenylallyl-ethyl-carbonat 198}

Gemäß AAV 4 wurde (E)-Zimtalkohol 197 (2.01 g, $15.0 \mathrm{mmol})$ in die Zielverbindung umgewandelt. Nach säulenchromatographischer Reinigung an Kieselgel (10 g, Pentan : Essigester = 30 : 1) wurden $2.13 \mathrm{~g}$ (10.6 mmol, 69\%) einer klaren Flüssigkeit erhalten.

$\mathbf{R}_{\mathbf{f}}=0.30($ Pentan : Essigester $=25: 1)$.

${ }^{1}$ H-NMR $\left(200 \mathrm{MHz}, \mathrm{CDCl}_{3}\right): \delta=1.32\left(\mathrm{t}, J=6.8 \mathrm{~Hz}, 3 \mathrm{H}, 2^{\prime}-\mathrm{H}_{3}\right), 4.23(\mathrm{q}, J=6.8 \mathrm{~Hz}, 2 \mathrm{H}$, $\left.1^{\prime}-\mathrm{H}_{2}\right), 4.79\left(\mathrm{~d}, J=6.3 \mathrm{~Hz}, 2 \mathrm{H}, 1-\mathrm{H}_{2}\right), 6.29$ (dt, $\left.J=16.2,6.3 \mathrm{~Hz}, 1 \mathrm{H}, 2-\mathrm{H}\right), 6.70$ (d, $J=16.2$ $\mathrm{Hz}, 1 \mathrm{H}, 3-\mathrm{H})$, 7.27-7.43 (m, $5 \mathrm{H}, \mathrm{Ph}-\mathrm{H})$.

${ }^{13}$ C-NMR (50.3 MHz, $\mathrm{CDCl}_{3}$ ): $\delta=14.28$ (C-2'), 64.05 (C-1'), 68.15 (C-1), 122.5 (C-2), 126.6 (C-5, C-9), 128.1 (C-7), 128.5 (C-6, C-8), 134.6 (C-3), 136.0 (C-4), 155.0 (C=O).

MS $\left(\mathrm{DCI}, \mathrm{NH}_{3}\right): \mathrm{m} / \mathrm{z}=224[\mathrm{M}+18]$.

$\mathrm{C}_{12} \mathrm{H}_{14} \mathrm{O}_{3}$ (206.23)

\subsection{3. (Z)-Ethyl-4-hydroxybut-2-encarbonat 275 und (Z)-1,4-Bis(ethoxycarbonyloxy)- but-2-en 276}

Gemäß AAV 4 wurde (Z)-But-2-en-1,4-diol 271 (3.28 ml, 3.52 g, 40.0 mmol) in die Zielverbindungen umgewandelt, wobei der Chlorameisensäureethylester sehr langsam zur Reaktionslösung hinzugegeben wurde. Nach säulenchromatographischer Trennung an Kieselgel (40 g, Pentan : Essigester = $3:$ 1) wurden $4.07 \mathrm{~g}$ (25.6 mmol, 64\%) 275 und $1.02 \mathrm{~g}$ (4.40 mmol, $11 \%) 276$ als farblose Flüssigkeiten erhalten.

275:

$\mathbf{R}_{\mathbf{f}}=0.80($ Pentan : Essigester $=3: 1)$.

${ }^{1}$ H-NMR $\left(200 \mathrm{MHz}, \mathrm{CDCl}_{3}\right): \delta=1.31\left(\mathrm{t}, J=7.3 \mathrm{~Hz}, 3 \mathrm{H}, 2^{\prime}-\mathrm{H}_{3}\right), 2.04(\mathrm{t}, J=6.5 \mathrm{~Hz}, 1 \mathrm{H}$, $\mathrm{OH}), 4.20\left(\mathrm{q}, J=7.3 \mathrm{~Hz}, 2 \mathrm{H}, 1^{\prime}-\mathrm{H}_{2}\right), 4.27\left(\mathrm{t}, J=6.5 \mathrm{~Hz}, 2 \mathrm{H}, 4-\mathrm{H}_{2}\right), 4.73(\mathrm{~d}, J=6.5 \mathrm{~Hz}, 2$ $\left.\mathrm{H}, 1-\mathrm{H}_{2}\right), 5.69(\mathrm{dt}, J=11.1,6.5 \mathrm{~Hz}, 1 \mathrm{H}, 3-\mathrm{H}), 5.91(\mathrm{dt}, J=11.1,6.5 \mathrm{~Hz}, 1 \mathrm{H}, 3-\mathrm{H})$.

${ }^{13}$ C-NMR (50.3 MHz, $\mathrm{CDCl}_{3}$ ): $\delta=14.24$ (C-2), 58.23 (C-4'), 63.09 (C-1), 64.22 (C-1'), 124.8 (C-2'), 134.0 (C-3'), $155.1(\mathrm{C}=\mathrm{O})$.

MS $\left(\mathrm{DCI}, \mathrm{NH}_{3}\right): \mathrm{m} / \mathrm{z}=178[\mathrm{M}+18]$.

$\mathrm{C}_{7} \mathrm{H}_{12} \mathrm{O}_{4}$ (160.16)

276:

$\mathbf{R}_{\mathbf{f}}=0.35($ Pentan : Essigester $=3: 1)$. 
${ }^{1} \mathbf{H}$-NMR $\left(200 \mathrm{MHz}, \mathrm{CDCl}_{3}\right): \delta=1.31\left(\mathrm{t}, J=7.5 \mathrm{~Hz}, 6 \mathrm{H}, 2^{\prime}-\mathrm{H}_{3}, 2\right.$ '’- $\left.\mathrm{H}_{3}\right), 4.21(\mathrm{q}, J=7 . \mathrm{Hz}$, $4 \mathrm{H}, 1$ '-H, 1' '-H), 4.74 (d, J=4.6 Hz, $\left.4 \mathrm{H}, 1-\mathrm{H}_{2}, 4-\mathrm{H}_{2}\right), 5.81(\mathrm{t}, J=4.6 \mathrm{~Hz}, 2 \mathrm{H}, 2-\mathrm{H}, 3-\mathrm{H})$.

${ }^{13}$ C-NMR (50.3 MHz, CDCl 3 ): $\delta=14.28$ (C-2', C-2'”), 62.97 (C-1', C-1'”), 64.15 (C-1, C4), $128.0(\mathrm{C}-2, \mathrm{C}-3), 154.9(\mathrm{C}=\mathrm{O})$.

MS $\left(\mathrm{DCI}, \mathrm{NH}_{3}\right): \mathrm{m} / \mathrm{z}=250[\mathrm{M}+18]$.

$\mathrm{C}_{10} \mathrm{H}_{16} \mathrm{O}_{6}(232.23)$

\subsection{Palladium-katalysierte allylische Alkylierungen in flüssiger Phase}

\subsubsection{2,2-Diallyl-acetessigsäuremethylester 206}

Gemäß AAV 5 wurde Acetessigsäuremethylester 205 (0.58 g, 5.0 mmol, 1 eq.) mit Essigsäureallylester 199 ( $1.1 \mathrm{ml}, 10 \mathrm{mmol}, 2.1$ eq.) für $30 \mathrm{~min}$ am Rückfluß erhitzt. Nach der Reinigung durch Säulenchromatographie an Kieselgel (20 g, Pentan : Essigester $=20: 1)$ erhielt man 0.86 g (4.4 mmol, 88\%) der Titelverbindung als klare Flüssigkeit.

$\mathbf{R}_{\mathbf{f}}=0.60($ Pentan : Essigester $=10: 1)$.

${ }^{1}$ H-NMR $\left(200 \mathrm{MHz} \mathrm{CDCl}_{3}\right): \delta=2.13\left(\mathrm{~s}, 3 \mathrm{H}, 4-\mathrm{H}_{3}\right), 2.62(\mathrm{dd}, J=7.4,2.9 \mathrm{~Hz}), 3.73(\mathrm{~s}, 3 \mathrm{H}$, $\mathrm{OMe}$ ), 5.08 (d, $J=9.6 \mathrm{~Hz}, 2 \mathrm{H}, 3$ '- $\mathrm{H}_{\mathrm{a}}, 3$ ' '- $\mathrm{H}_{\mathrm{a}}$ ), 5.12 (d, $J=17.4 \mathrm{~Hz}, 2 \mathrm{H}, 3$ ' $-\mathrm{H}_{\mathrm{b}}, 3$ ' '- $-\mathrm{H}_{\mathrm{b}}$ ), $5.47-$ 5.71 (ddt, $J=17.4 \mathrm{~Hz}, 9.6,7.4 \mathrm{~Hz}, 2 \mathrm{H}, 2$ '-H, 2'’-H).

${ }^{13}$ C-NMR (50.3 MHz, $\mathrm{CDCl}_{3}$ ): $\delta=27.31(\mathrm{C}-4), 36.03$ (C-1', C-1'’), $52.31(\mathrm{OMe}), 63.37$ (C2), 119.4 (C-3', C-3'”), 131.9 (C-2', C-2'’), 171.13 (C-1), 203.1 (C-3).

MS (DCI, $\left.\mathrm{NH}_{3}\right): \mathrm{m} / \mathrm{z}=214[\mathrm{M}+18]$.

$\mathrm{C}_{11} \mathrm{H}_{16} \mathrm{O}_{3}$ (196.24)

\subsubsection{2-(2-Cyclohex-2-enyl)-acetessigsäuremethylester 260}

Gemäß AAV 5 wurde Acetessigsäuremethylester 205 (58 mg, 0.50 mmol, 1 eq.) mit Essigsäure-cyclopent-2-enylester $175(0.14 \mathrm{mg}, 1.0 \mathrm{mmol}, 2.1 \mathrm{eq}$.) für $30 \mathrm{~min}$ am Rückfluß erhitzt. Säulenchromatographische Reinigung an Kieselgel (5 g, Pentan : Essigester $=20: 1)$ ergab $72 \mathrm{mg}(0.36 \mathrm{mmol}, 73 \%)$ der Titelverbindung als farblose Flüssigkeit.

$\mathbf{R}_{\mathbf{f}}=0.55($ Pentan : Essigester $=10: 1)$. 
${ }^{1}$ H-NMR (200 MHz, $\left.\mathrm{CDCl}_{3}\right): \delta=1.44-1.90\left(\mathrm{~m}, 4 \mathrm{H}, 5^{\prime}-\mathrm{H}_{2}, 6^{\prime}-\mathrm{H}_{2}\right), 2.00\left(\mathrm{~m}_{\mathrm{c}}, 2 \mathrm{H}, 4^{\prime}-\mathrm{H}_{2}\right), 2.25$ (s, 3 H, 4-H H, 2.96 (mc, 1 H, 1'-H), 3.39 (d, J = 9.5 Hz, 1 H, 2-H), 3.74 (s, 3 H, OMe), 5.375.54 (m, 1 H, 3'-H), 5.71-5.84 (m, 1 H, 2'-H).

${ }^{13}$ C-NMR (50.3 MHz, CDCl $)$ ): $\delta=20.77$ (C-4'), 24.9 (C-5'), 26.65 (C-6'), 29.63 (C-4), 35.13 (C-1'), 52.33 (OMe), 65.06 (C-2), 127.1 (C-2'), 129.6 (C-3'), 169.2 (C-1), 202.5 (C-3). MS $\left(\mathrm{DCI}, \mathrm{NH}_{3}\right): \mathrm{m} / \mathrm{z}=197[\mathrm{M}+1], 214[\mathrm{M}+18]$.

$\mathrm{C}_{11} \mathrm{H}_{16} \mathrm{O}_{3}$ (196.24)

\subsection{3. (E)-2-(4-Hydroxybut-2-enyl)-acetessigsäuremethylester 277 und (E)-2,2-Bis(4- hydroxybut-2-enyl)-acetessigsäuremethylester 278}

In Anlehnung an AAV 5 wurde Acetessigsäuremethylester 205 (58 mg, 0.50 mmol, 1 eq.) mit (Z)-Ethyl-4-hydroxybut-2-encarbonat 275 ( $0.16 \mathrm{mg}, 1.0 \mathrm{mmol}, 2$ eq.) $1 \mathrm{~h}$ bei Raumtemperatur gerührt. Nach Einengen im Vakuum und Reinigung durch Säulenchromatographie an Kieselgel (5 g, Et $\left.{ }_{2} \mathrm{O}\right)$ erhielt man $46 \mathrm{mg}(0.24 \mathrm{mmol}, 49 \%) 277$ und $51 \mathrm{mg}(0.20 \mathrm{mmol}, 40 \%) 278$ als klare Öle.

277:

$\mathbf{R}_{\mathbf{f}}=0.43\left(\mathrm{Et}_{2} \mathrm{O}\right)$.

${ }^{1}$ H-NMR $\left(200 \mathrm{MHz}, \mathrm{CDCl}_{3}\right.$ ): $\delta=1.26$ (br. s, $\left.1 \mathrm{H}, \mathrm{OH}\right), 2.24$ (s, $\left.3 \mathrm{H}, 4-\mathrm{H}_{3}\right), 2.61$ (t, $J=7.5$ $\mathrm{Hz}, 2 \mathrm{H}, 1$ ' $\left.-\mathrm{H}_{2}\right), 3.53$ (t, J=7.5 Hz, $\left.1 \mathrm{H}, 2-\mathrm{H}\right), 3.75$ (s, $\left.3 \mathrm{H}, \mathrm{OMe}\right), 4.04$ (d, J = 4.7 Hz, $2 \mathrm{H}$, 4'- $\left.\mathrm{H}_{2}\right), 5.56-5.82$ (m, 2 H, 2'-H, 3'-H).

${ }^{13}$ C-NMR (75.4 MHz, $\left.\mathrm{CDCl}_{3}\right): \delta=29.20(\mathrm{C}-4), 30.68\left(\mathrm{C}-1^{\prime}\right), 52.51(\mathrm{OMe}), 59.18(\mathrm{C}-2)$, 63.18 (C-4'), 127.6 (C-3'), 132.2 (C-2'), 164.6 (C-1), 202.3 (C=O).

MS $\left(\mathrm{DCI}, \mathrm{NH}_{3}\right): \mathrm{m} / \mathrm{z}=204[\mathrm{M}+18]$.

$\mathrm{C}_{9} \mathrm{H}_{14} \mathrm{O}_{4}$ (186.20)

278:

$\mathbf{R}_{\mathbf{f}}=0.15\left(\mathrm{Et}_{2} \mathrm{O}\right)$.

${ }^{1} \mathbf{H}-\mathbf{N M R}\left(200 \mathrm{MHz}, \mathrm{CDCl}_{3}\right): \delta=2.15$ (s, $\left.3 \mathrm{H}, 4-\mathrm{H}_{3}\right), 2.56-2.69$ (m, $4 \mathrm{H}, 1$ ' - $\mathrm{H}_{2}, 1$ '’ $\left.-\mathrm{H}_{2}\right), 3.75$ (s, 3 H, OMe), 4.04-4.21 (m, 4 H, 4'-H $\mathrm{H}_{2}$ ' '- $-\mathrm{H}_{2}$ ), 5.19-5.80 (m, 4 H, 2'-H, 3'-H, 2' '-H, 3' '-H). ${ }^{13} \mathbf{C}-N M R\left(50.3 \mathrm{MHz}, \mathrm{CDCl}_{3}\right): \delta=26.80(\mathrm{C}-4), 34.47$ (C-1', C-1'”), $52.64(\mathrm{OMe}), 58.03(\mathrm{C}-$ 2), 63.03 (C-2', C-2'’), 171.3 (C-1), $204.0(\mathrm{C}=\mathrm{O})$.

MS (DCI, $\left.\mathrm{NH}_{3}\right): \mathrm{m} / \mathrm{z}=274[\mathrm{M}+18]$.

$\mathrm{C}_{13} \mathrm{H}_{20} \mathrm{O}_{5}$ (256.29) 


\section{Synthesen an der festen Phase}

\subsection{Synthese von spacermodifiziertem Harz 6}

Natriumhydrid (5.00 g, $125 \mathrm{mmol}$, 60\%ig in Mineralöl, 5 eq.) wurde in trockenem DMF $(50 \mathrm{ml})$ suspendiert und unter Eiskühlung langsam mit einer Lösung von 1,3-Propandiol ( $9.03 \mathrm{ml}, 9.51 \mathrm{~g}, 125 \mathrm{mmol}, 5$ eq.) in DMF (50 ml) versetzt. Nachdem zwei Stunden bei Raumtemperatur gerührt wurde, erfolgte die Zugabe von $n$-Tetrabutylammoniumiodid $(0.92 \mathrm{~g}$, $2.5 \mathrm{mmol}, 0.1$ eq.) und Merrifield-Harz (10 g, $25 \mathrm{mmol}, 1$ eq., 2\% DVB, $2.5 \mathrm{mmol} \mathrm{Cl} / \mathrm{g}$, ACROS). Falls erforderlich wurde die Suspension zur besseren Rührbarkeit mit weiterem DMF versetzt. Das Gemisch wurde 24 Stunden bei Raumtemperatur unter Lichtausschluß gerührt. Anschließend kühlte man auf $0{ }^{\circ} \mathrm{C}$, gab Wasser $(100 \mathrm{ml})$ hinzu, filtriert das Harz und wusch gründlich mit DMF (100 ml), MeOH $(100 \mathrm{ml})$ und $\mathrm{CH}_{2} \mathrm{Cl}_{2}(200 \mathrm{ml})$. Anschließend trocknete man das Harz bei $55^{\circ} \mathrm{C}$ im Vakuum.

\subsection{Synthese des polymergebundenen 3,5-Dinitrobenzoesäureesters 131}

Spacermodifiziertes Harz 6 (50 mg, 1 eq.) wurde in Pyridin $(2 \mathrm{ml})$ suspendiert, mit 3,5Dinitrobenzoylchlorid 130 ( $86 \mathrm{mg}, 0.37 \mathrm{mmol}, 10$ eq.) versetzt und für eine Stunde bei $85^{\circ} \mathrm{C}$ gerührt. Nach dem Abkühlen auf Raumtemperatur gab man Wasser $(3 \mathrm{ml})$ hinzu, filtrierte das Harz ab, wusch gründlich mit DMF (10 ml), MeOH $(10 \mathrm{ml})$ und $\mathrm{CH}_{2} \mathrm{Cl}_{2}(10 \mathrm{ml})$ und trocknete das Harz bei $55^{\circ} \mathrm{C}$ im Vakuum.

IR (KBr): $\widetilde{v}=1732(\mathrm{C}=\mathrm{O}), 1510,1340\left(\mathrm{NO}_{2}\right) \mathrm{cm}^{-1} .^{[68]}$

Elementaranalyse: $2.1-2.4 \%$ Stickstoff.

\subsection{Synthese von polymergebundener Benzoesäure 133}

In Anlehnung an AAV 3 wurde spacermodifiziertes Harz 6 (300 mg, 1 eq.) mit Benzoylchlorid 132 (10 eq.) umgesetzt.

IR $(\mathrm{KBr}): \widetilde{\mathbf{v}}=1718(\mathrm{C}=\mathrm{O}) \mathrm{cm}^{-1} \cdot{ }^{[68]}$ 


\subsection{Synthese von polymergebundenem Acetoacetat 138}

Spacermodifiziertes Harz 6 (10.0 g, 1 eq.) wurde in Toluol $(50 \mathrm{ml})$ suspendiert, mit Acetessigsäure-t-butylester 135 (12.2 ml, $12.3 \mathrm{~g}, 75.0 \mathrm{mmol}, 10 \mathrm{eq.})$ versetzt und für 3 Stunden am Rückfluß erhitzt. Nach dem Abkühlen der Reaktionslösung auf Raumtemperatur wurde das Harz filtriert, gründlich mit $\mathrm{MeOH}(100 \mathrm{ml})$ und $\mathrm{CH}_{2} \mathrm{Cl}_{2}(100 \mathrm{ml})$ gewaschen und bei $55^{\circ} \mathrm{C} \mathrm{im}$ Vakuum getrocknet.

IR $(\mathrm{KBr}): \widetilde{v}=1742$ (Ester), $1718($ Keton $) \mathrm{cm}^{-1} \cdot{ }^{[68]}$

\subsection{Synthese von polymergebundenem Methylmalonat 143}

Spacermodifiziertes Harz 6 (3 g, 1 eq.) wurde in trockenem $\mathrm{CH}_{2} \mathrm{Cl}_{2}(15 \mathrm{ml})$ suspendiert und mit $\mathrm{NEt}_{3}(1.55 \mathrm{ml}, 1.13 \mathrm{~g}, 11.2 \mathrm{mmol}, 5$ eq. $)$ versetzt. Bei $0{ }^{\circ} \mathrm{C}$ gab man tropfenweise Malonsäuremethylesterchlorid $142(1.21 \mathrm{ml}, 1.54 \mathrm{~g}, 11.2 \mathrm{mmol}, 5 \mathrm{eq}$.$) hinzu und rührte für$ 3 Stunden bei $0{ }^{\circ} \mathrm{C}$ und für $1 \mathrm{~h}$ bei Raumtemperatur. Anschleißend wurde das Harz filtriert, gründlich mit $\mathrm{MeOH}(50 \mathrm{ml})$ und $\mathrm{CH}_{2} \mathrm{Cl}_{2}(50 \mathrm{ml})$ gewaschen und bei $55^{\circ} \mathrm{C}$ im Vakuum getrocknet.

IR $(\mathrm{KBr}): \widetilde{v}=1734($ Ester $) \mathrm{cm}^{-1}{ }^{[68]}$

\subsection{Allylische Alkylierungen mit Allylacetaten an der festen Phase}

\subsubsection{2,2-Diallyl-butan-1,3-diol 202}

Gemäß AAV 6 wurde polymergebundenes Acetoacetat 138 (300 mg) mit Essigsäureallylester 1995 h am Rückfluß erhitzt und gemäß AAV 8 das erhaltene Produkt von der festen Phase abgespalten. Nach säulenchromatographischer Reinigung an Kieselgel (5 g, Pentan : Essigester $=5: 1)$ erhielt man $31 \mathrm{mg}(0.18 \mathrm{mmol}, 88 \%)$ eines farblosen Öls.

$\mathbf{R}_{\mathbf{f}}=0.55$ (Diethylether).

IR (Film): $\widetilde{v}=3350(\mathrm{OH}), 3005,2958(\mathrm{CH}), 1642(\mathrm{C}=\mathrm{C}), 1451,1378\left(\mathrm{CH}_{3}\right) \mathrm{cm}^{-1}$.

$\mathbf{U V}\left(\mathrm{CH}_{3} \mathrm{CN}\right): \lambda_{\max }(\lg \varepsilon)=233.5(2.887) \mathrm{nm}$.

${ }^{1}$ H-NMR $\left(200 \mathrm{MHz}, \mathrm{CDCl}_{3}\right): \delta=1.26\left(\mathrm{~d}, J=6.2 \mathrm{~Hz}, 3 \mathrm{H}, 4-\mathrm{H}_{3}\right), 1.92(\mathrm{dd}, J=14.1,7.4 \mathrm{~Hz}$, $\left.1 \mathrm{H}, 1^{\prime}-\mathrm{H}_{\mathrm{a}}\right), 2.19$ (dd, $\left.J=14.1,7.4 \mathrm{~Hz}, 1 \mathrm{H}, 1^{\prime}-\mathrm{H}_{\mathrm{b}}\right), 2.28$ (d, J = 7.4 Hz, $2 \mathrm{H}, 1$ '’-H), 2.35 (br. 
s, $2 \mathrm{H}, \mathrm{OH}), 3.57\left(\mathrm{~d}, J=11.0 \mathrm{~Hz}, 1 \mathrm{H}, 1-\mathrm{H}_{\mathrm{a}}\right), 3.75\left(\mathrm{~d}, J=11.0 \mathrm{~Hz}, 1 \mathrm{H}, 1-\mathrm{H}_{\mathrm{b}}\right), 3.88(\mathrm{q}, J=$ $6.2 \mathrm{~Hz}, 1 \mathrm{H}, 3-\mathrm{H}), 5.08$ (d, J=11.5 Hz, $2 \mathrm{H}, 3^{\prime}-\mathrm{H}_{\mathrm{a}}, 3^{\prime}$ ' $-\mathrm{H}_{\mathrm{a}}$ ), 5.12 (d, J = 15.1 Hz, $2 \mathrm{H}, 3^{\prime}-\mathrm{H}_{\mathrm{b}}$, 3',-H $\mathrm{H}_{\mathrm{b}}, 5.86$ (ddt, $J=15.1,11.5,6.2 \mathrm{~Hz}, 2 \mathrm{H}, 2$ '-H, 2' '-H).

${ }^{13}$ C-NMR (50.3 MHz, $\left.\mathrm{CDCl}_{3}\right): \delta=18.04$ (C-4), 36.14 (C-1', C-1'”), 45.47 (C-2), 67.03 (C1), 73.87 (C-3), 118.1 (C-3', C-3'’), 133.9 (C-2', C-2'’).

MS $\left(\mathrm{DCI}, \mathrm{NH}_{3}\right): \mathrm{m} / \mathrm{z}=188[\mathrm{M}+18], 205[\mathrm{M}+18+17]$.

$\mathrm{C}_{10} \mathrm{H}_{18} \mathrm{O}_{2}$ (170.24)

\subsubsection{2,2-Diallyl-propan-1,3-diol 203}

Gemäß AAV 6 wurde polymergebundenes Methylmalonat 143 (300 mg) mit Essigsäureallylester $1995 \mathrm{~h}$ am Rückfluß erhitzt und gemäß AAV 8 das erhaltene Produkt von der festen Phase abgespalten. Nach säulenchromatographischer Reinigung an Kieselgel (5 g, Pentan : Essigester $=5: 1)$ erhielt man $26 \mathrm{mg}(0.16 \mathrm{mmol}, 79 \%)$ eines farblosen Öls.

$\mathbf{R}_{\mathbf{f}}=0.50$ (Diethylether).

IR (Film): $\widetilde{v}=3384(\mathrm{OH}), 2952,2927(\mathrm{CH}), 1639(\mathrm{C}=\mathrm{C}) \mathrm{cm}^{-1}$.

$\mathbf{U V}\left(\mathrm{CH}_{3} \mathrm{CN}\right): \lambda_{\max }(\lg \varepsilon)=234.7(2.934) \mathrm{nm}$.

${ }^{1} \mathbf{H}$-NMR $\left(200 \mathrm{MHz}, \mathrm{CDCl}_{3}\right): \delta=2.08\left(\mathrm{~d}, J=7.0 \mathrm{~Hz}, 4 \mathrm{H}, 1^{\prime}-\mathrm{H}_{2}, 1\right.$ '’- $\left.\mathrm{H}_{2}\right), 2.74$ (br. s, $2 \mathrm{H}$, $\mathrm{OH}), 3.58\left(\mathrm{~s}, 4 \mathrm{H}, 1-\mathrm{H}_{2}, 3-\mathrm{H}_{2}\right), 5.07$ (d, $J=11.8 \mathrm{~Hz}, 2 \mathrm{H}, 3^{\prime}-\mathrm{H}_{\mathrm{a}}, 3^{\prime}$ ' $\left.-\mathrm{H}_{\mathrm{a}}\right), 5.10$ (d, $J=15.6 \mathrm{~Hz}$, $2 \mathrm{H}, 3$ '- $\mathrm{H}_{\mathrm{b}}, 3$ ''- $\mathrm{H}_{\mathrm{b}}$ ), 5.84 (ddt, $J=15.6,11.8,7.0 \mathrm{~Hz}, 2 \mathrm{H}, 2$ '-H, 2''-H).

${ }^{13} \mathrm{C}-\mathrm{NMR}\left(50.3 \mathrm{MHz}, \mathrm{CDCl}_{3}\right): \delta=36.04\left(\mathrm{C}-1\right.$ ', C-1' $\left.{ }^{\prime}\right), 42.05(\mathrm{C}-2), 68.13(\mathrm{C}-1, \mathrm{C}-3), 118.1$ (C-3', C-3''), 133.8 (C-2', C-2'').

MS (DCI, $\left.\mathrm{NH}_{3}\right): \mathrm{m} / \mathrm{z}=174$ [M+18], 191 [M+18+17], 330 [2M+18].

$\mathrm{C}_{9} \mathrm{H}_{16} \mathrm{O}_{2}(156.22)$

\subsection{3. (E)-2,2-Dibut-2-enyl-butan-1,3-diol 210}

Gemäß AAV 6 wurde polymergebundenes Acetoacetat 138 (350 mg) mit (E/Z)-Essigsäurebut-2-enyl-ester $1565 \mathrm{~h}$ am Rückfluß erhitzt und gemäß AAV 8 das erhaltene Produkt von der festen Phase abgespalten. Nach säulenchromatographischer Reinigung an Kieselgel (5 g, Pen$\tan :$ Essigester $=5: 1)$ erhielt man $16 \mathrm{mg}(0.082 \mathrm{mmol}, 39 \%)$ eines farblosen Öls.

$\mathbf{R}_{\mathbf{f}}=0.65$ (Diethylether).

IR (Film): $\widetilde{v}=3374(\mathrm{OH}), 3025,2978(\mathrm{CH}), 1647(\mathrm{C}=\mathrm{C}), 1449,1376\left(\mathrm{CH}_{3}\right) \mathrm{cm}^{-1}$. 
$\mathbf{U V}\left(\mathrm{CH}_{3} \mathrm{CN}\right): \lambda_{\max }(\lg \varepsilon)=232.0(3.056) \mathrm{nm}$.

${ }^{1}$ H-NMR $\left(200 \mathrm{MHz}, \mathrm{CDCl}_{3}\right): \delta=1.23\left(\mathrm{~d}, J=6.7 \mathrm{~Hz}, 3 \mathrm{H}, 4-\mathrm{H}_{3}\right), 1.68(\mathrm{~d}, J=3.9 \mathrm{~Hz}, 6 \mathrm{H}$, 4' $-\mathrm{H}_{3}, 4$ ' '- $\mathrm{H}_{3}$ ), 1.75-2.13 (m, $\left.2 \mathrm{H}, 1^{\prime}-\mathrm{H}_{2}\right), 2.18$ (d, J = 5.5 Hz, $2 \mathrm{H}, 1$ '’ $-\mathrm{H}_{2}$ ), 2.54 (br. s, $2 \mathrm{H}$, $\mathrm{OH}), 3.52\left(\mathrm{dd}, J=11.7,4.3 \mathrm{~Hz}, 1 \mathrm{H}, 1-\mathrm{H}_{\mathrm{a}}\right), 3.70\left(\mathrm{dd}, J=11.7,4.3 \mathrm{~Hz}, 1 \mathrm{H}, 1-\mathrm{H}_{\mathrm{b}}\right), 3.85\left(\mathrm{~m}_{\mathrm{c}}\right.$, $1 \mathrm{H}, 3-\mathrm{H}), 5.41-5.64$ (m, 4 H, 2'-H, 3'-H, 2' -H, 3' '-H).

${ }^{13}$ C-NMR (200 MHz, $\left.\mathrm{CDCl}_{3}\right): \delta=17.80$ (C-4), 17.9 (C-4', C-4'’), 34.04, 35.37 (C-1', C-1'’), 43.9 (C-2), 67.68 (C-1), 73.54 (C-3), 126.5 (C-3', C-3'’), 128.3 (C-2', C-2'’).

MS (DCI, $\left.\mathrm{NH}_{3}\right): \mathrm{m} / \mathrm{z}=216$ [M+18], 233 [M+18+17], 414 [2M+18].

$\mathrm{C}_{12} \mathrm{H}_{22} \mathrm{O}_{2}$ (198.30)

\subsubsection{2,2-Bis-(2-methyl-allyl)-butan-1,3-diol 211}

Gemäß AAV 6 wurde polymergebundenes Acetoacetat 138 (350 mg) mit Essigsäure-2methylallylester $1585 \mathrm{~h}$ am Rückfluß erhitzt und gemäß AAV 8 das erhaltene Produkt von der festen Phase abgespalten. Nach säulenchromatographischer Reinigung an Kieselgel (5 g, Pen$\tan :$ Essigester $=5: 1)$ erhielt man $25 \mathrm{mg}(0.12 \mathrm{mmol}, 60 \%)$ eines farblosen Öls.

$\mathbf{R}_{\mathbf{f}}=0.70$ (Diethylether).

IR (Film): $\widetilde{v}=3372(\mathrm{OH}), 2994,2962(\mathrm{CH}), 1655(\mathrm{C}=\mathrm{C}), 1448,1377\left(\mathrm{CH}_{3}\right) \mathrm{cm}^{-1}$.

$\mathbf{U V}\left(\mathrm{CH}_{3} \mathrm{CN}\right): \lambda_{\max }(\lg \varepsilon)=228.0(3.147) \mathrm{nm}$.

${ }^{1}$ H-NMR $\left(200 \mathrm{MHz}, \mathrm{CDCl}_{3}\right): \delta=1.26\left(\mathrm{~d}, J=6.2 \mathrm{~Hz}, 3 \mathrm{H}, 4-\mathrm{H}_{3}\right), 1.83$ (s, $3 \mathrm{H}, 2$ ' $\left.-\mathrm{CH}_{3}\right), 1.86$ (s, $3 \mathrm{H}, 2$ ' ' $-\mathrm{CH}_{3}$ ), 2.00 (d, J=13.6 Hz, $2 \mathrm{H}, 1$ ' $-\mathrm{H}_{\mathrm{a}}, 1$ ' ' $-\mathrm{H}_{\mathrm{a}}$ ), 2.42 (d, J=13.6 Hz, $2 \mathrm{H}, 1$ '- $\mathrm{H}_{\mathrm{a}}$, 1 ' '- $\mathrm{H}_{\mathrm{b}}$ ), 2.56 (br. s, $2 \mathrm{H}, \mathrm{OH}$ ), 3.76 (br. s, $2 \mathrm{H}, 1-\mathrm{H}_{2}$ ), 4.14 (q, $J=6.2 \mathrm{~Hz}, 1 \mathrm{H}, 3-\mathrm{H}$ ), 4.794.96 (m, 4 H, 3'- $\mathrm{H}_{2}, 3^{\prime}$ ' $\left.-\mathrm{H}_{2}\right)$.

${ }^{13} \mathbf{C}-\mathrm{NMR}\left(50.3 \mathrm{MHz}, \mathrm{CDCl}_{3}\right): \delta=18.29(\mathrm{C}-4), 24.19$ (2'- $\mathrm{CH}_{3}, 2$ ''- $\left.\mathrm{CH}_{3}\right), 38.05$ (C-1', C-1' '), 44.59 (C-2), 67.12 (C-1), 71.96 (C-3), 115.0 (C-3', C-3'’), 143.8 (C-2', C-2' ').

MS (DCI, $\left.\mathrm{NH}_{3}\right): \mathrm{m} / \mathrm{z}=216[\mathrm{M}+18], 233[\mathrm{M}+18+17]$.

$\mathrm{C}_{11} \mathrm{H}_{22} \mathrm{O}_{2}$ (198.30)

\subsubsection{2,2-Bis(2-methyl-allyl)-propan-1,3-diol 212}

Gemäß AAV 6 wurde polymergebundenes Methylmalonat 143 (350 mg) mit Essigsäure-2methylallylester 1585 h am Rückfluß erhitzt und gemäß AAV 8 das erhaltene Produkt von der festen Phase abgespalten. Nach säulenchromatographischer Reinigung an Kieselgel (5 g, Pen$\tan$ : Diethylether $=2: 1)$ erhielt man $17 \mathrm{mg}(0.092 \mathrm{mmol}, 37 \%)$ eines farblosen Öls. 
$\mathbf{R}_{\mathbf{f}}=0.60$ (Diethylether).

IR (Film): $\widetilde{v}=3372(\mathrm{OH}), 3020,2971(\mathrm{CH}), 1648(\mathrm{C}=\mathrm{C}) \mathrm{cm}^{-1}$.

UV $\left(\mathrm{CH}_{3} \mathrm{CN}\right): \lambda_{\max }(\lg \varepsilon)=232.0(3.254) \mathrm{nm}$.

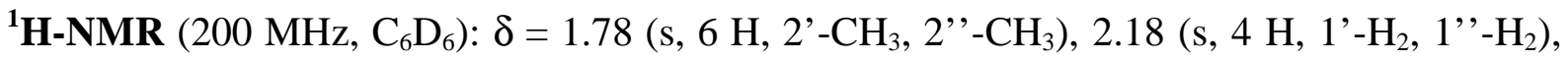
3.54 (s, 4 H, 1- $\left.\mathrm{H}_{2}, 3-\mathrm{H}_{2}\right), 4.84$ (m, $\left.2 \mathrm{H}, 3^{\prime}-\mathrm{H}_{2}\right), 4.93$ (m, $2 \mathrm{H}, 3$ ' '- $\left.\mathrm{H}_{2}\right)$.

${ }^{13}$ C-NMR (75.5 MHz, $\mathrm{C}_{6} \mathrm{D}_{6}$ ): $\delta=25.43$ (2'-C, 2'’-C), 41.00 (C-1', C-1'’), 43.56 (C-2), 67.96 (C-1, C-3), 115.1 (C-3', C-3''), 143.33 (C-2', C-2'').

MS (DCI, $\left.\mathrm{NH}_{3}\right): \mathrm{m} / \mathrm{z}=202[\mathrm{M}+18], 219[\mathrm{M}+18+17]$.

$\mathrm{C}_{11} \mathrm{H}_{20} \mathrm{O}_{2}$ (184.27)

\subsection{6. (E)-2-(3-Methyl-5-(2,5-dimethoxy-3,4,6-trimethylphenyl)-pent-2-enyl)-butan- 1,3-diol 213}

Gemäß AAV 6 wurde polymergebundenes Acetoacetat 138 (300 mg) mit (E)-Essigsäure-5(2,5-dimethoxy-3,4,6-trimethylphenyl)-3-methyl-pent-2-enylester $1595 \mathrm{~h}$ am Rückfluß erhitzt und gemäß AAV 8 das erhaltene Produkt von der festen Phase abgespalten. Nach Reinigung an Kieselgel ( 5 g, Pentan : Essigester $=5: 1)$ wurden $5 \mathrm{mg}(0.02 \mathrm{mmol}, 8 \%)$ eines klaren Öls erhalten.

$\mathbf{R}_{\mathbf{f}}=0.30$ (Diethylether).

IR (Film): $\widetilde{\mathrm{v}}=3381(\mathrm{OH}), 2935(\mathrm{CH}), 1659,1651(\mathrm{C}=\mathrm{C}), 1455,1378\left(\mathrm{CH}_{3}\right) \mathrm{cm}^{-1}$.

UV $\left(\mathrm{CH}_{3} \mathrm{CN}\right): \lambda_{\max }(\lg \varepsilon)=217.5$ (3.958), 255.1 (3.541), $289.2(3.487) \mathrm{nm}$.

${ }^{1} \mathbf{H}$-NMR $\left(200 \mathrm{MHz}, \mathrm{CDCl}_{3}\right): \delta=1.28\left(\mathrm{~d}, J=6.7 \mathrm{~Hz}, 3 \mathrm{H}, 4-\mathrm{H}_{3}\right), 1.73$ (br. s, $5 \mathrm{H}, 3$ '- $\mathrm{CH}_{3}$, $\mathrm{OH}), 1.95-2.26$ (m, $\left.4 \mathrm{H}, 1^{\prime}-\mathrm{H}_{2}, 4^{\prime}-\mathrm{H}_{2}\right), 2.18$ (s, 9 H, 8'- $\left.\mathrm{CH}_{3}, 11^{\prime}-\mathrm{CH}_{3}\right), 2.63-2.75$ (m, $2 \mathrm{H}, 5^{\prime}-$ $\mathrm{H}_{2}$ ), 3.57-3.83 (m, $\left.3 \mathrm{H}, 1-\mathrm{H}_{2}, 3-\mathrm{H}\right), 3.64$ (s, $\left.3 \mathrm{H}, \mathrm{OMe}\right), 3.68$ (s, $\left.3 \mathrm{H}, \mathrm{OMe}\right), 5.16$ (t, $J=6.9$ $\left.\mathrm{Hz}, 1 \mathrm{H}, 2^{\prime}-\mathrm{H}\right)$.

${ }^{13}$ C-NMR (50.3 MHz, $\left.\mathrm{CDCl}_{3}\right): \delta=12.03$ (9'-C), 12.71 (8'-C), 13.11 (11'-C), 16.56 (3'-C), 21.43 (C-1'), 22.58 (C-4), 26.09 (C-5'), 39.91 (C-4'), 50.63 (C-2), 59.87, 60.96 (OMe), 62.87 (C-1), 70.01 (C-3), 118.1 (C-2'), 127.1 (C-11'), 127.9 (C-8'), 128.3 (C-9'), 131.6 (C6'), 142.4 (C-3'), 152.9 (C-7'), 153.0 (C-10').

MS (DCI, $\left.\mathrm{NH}_{3}\right): \mathrm{m} / \mathrm{z}=351[\mathrm{M}+1], 368$ [M+18], 718 [2M+18].

$\mathrm{C}_{21} \mathrm{H}_{34} \mathrm{O}_{4}$ (350.49) 


\subsubsection{2,2-Diallyl-4-phenyl-butan-1,3-diol 219}

Gemäß AAV 6 wurde polymergebundenes Phenylacetoacetat 218 (300 mg) mit Essigsäureallylester $1995 \mathrm{~h}$ am Rückfluß erhitzt und gemäß AAV 8 das erhaltene Produkt von der festen Phase abgespalten. Nach säulenchromatographischer Reinigung an Kieselgel (5 g, Pentan : Essigester $=5: 1)$ erhielt man $36 \mathrm{mg}(0.15 \mathrm{mmol}, 73 \%)$ eines farblosen Öls.

$\mathbf{R}_{\mathbf{f}}=0.75$ (Diethylether).

IR (Film): $\widetilde{\mathrm{v}}=3376(\mathrm{OH}), 2975(\mathrm{CH}), 1673,1638(\mathrm{C}=\mathrm{C}) \mathrm{cm}^{-1}$.

UV $\left(\mathrm{CH}_{3} \mathrm{CN}\right): \lambda_{\max }(\lg \varepsilon)=235.0(2.565) \mathrm{nm}$.

${ }^{1} \mathbf{H}$-NMR $\left(200 \mathrm{MHz}, \mathrm{CDCl}_{3}\right): \delta=1.85\left(\mathrm{dd}, J=14.5,7.7 \mathrm{~Hz}, 1 \mathrm{H}, 1\right.$ '- $\left.\mathrm{H}_{\mathrm{a}}\right), 1.99(\mathrm{dd}, J=14.5$, $7.7 \mathrm{~Hz}, 1 \mathrm{H}, 1$ ' $\left.-\mathrm{H}_{\mathrm{b}}\right), 2.26$ (dd, $\left.J=14.3,7.4 \mathrm{~Hz}, 1 \mathrm{H}, 1^{\prime}-\mathrm{H}_{\mathrm{a}}\right), 2.48(\mathrm{dd}, J=14.3,7.4 \mathrm{~Hz}, 1 \mathrm{H}$, 1' $\left.-\mathrm{H}_{\mathrm{b}}\right), 2.71$ (t, $\left.J=6.1 \mathrm{~Hz}, 1 \mathrm{H}, \mathrm{OH}\right), 3.02(\mathrm{~d}, J=4.0 \mathrm{~Hz}, 1 \mathrm{H}, \mathrm{OH}), 3.52$ (dd, $J=11.4,6.1$ $\left.\mathrm{Hz}, 1 \mathrm{H}, 3-\mathrm{H}_{\mathrm{a}}\right), 3.67$ (dd, $\left.J=11.4,6.1 \mathrm{~Hz}, 1 \mathrm{H}, 3-\mathrm{H}_{\mathrm{b}}\right), 4.80$ (d, $\left.J=4.0 \mathrm{~Hz}, 1 \mathrm{H}, 1-\mathrm{H}\right), 4.95-$ $5.27\left(\mathrm{~m}_{\mathrm{c}}, 4 \mathrm{H}, 3\right.$ '- $\mathrm{H}_{2}, 3$ '' $\left.-\mathrm{H}_{2}\right)$, 5.71-6.08 ( $\mathrm{m}, 2$ H, 2'-H, 2''-H), 7.28-7.45 (m, 5 H, Ph-H).

${ }^{13}$ C-NMR (50.3 MHz, $\mathrm{CDCl}_{3}$ ): $\delta=35.69,36.04$ (C-1', C-1','), 44.18 (C-2), 67.03 (C-3), 79.94 (C-1), 118.2, 118.3 (C-3'’, C-3'’'), 127.6 (C-4'), 127.6, 127.8 (C-3', C-5'), 127.7, 127.9 (C-2', C-2','), 134.0, 134.1 (C-2', C-6'), 140.9 (C-1').

MS (DCI, $\left.\mathrm{NH}_{3}\right): \mathrm{m} / \mathrm{z}=250$ [M+18], 267 [M+18+17], 482 [2M+18].

$\mathrm{C}_{15} \mathrm{H}_{20} \mathrm{O}_{2}$ (232.31)

\subsubsection{2-Cyclohex-2-enyl-butan-1,3-diol 227}

Gemäß AAV 6 wurde polymergebundenes Acetoacetat 138 (300 mg) mit Essigsäurecyclohex-2-enylester $1755 \mathrm{~h}$ am Rückfluß erhitzt und gemäß AAV 8 das erhaltene Produkt von der festen Phase abgespalten. Nach säulenchromatographischer Reinigung an Kieselgel (4 g, Pentan : Essigester $=10: 1)$ erhielt man $21 \mathrm{mg}(0.12 \mathrm{mmol}, 59 \%)$ eines farblosen Öls.

$\mathbf{R}_{\mathbf{f}}=0.50$ (Diethylether).

IR (Film): $\widetilde{v}=3377(\mathrm{OH}), 2968,2931(\mathrm{CH}), 1652(\mathrm{C}=\mathrm{C}), 1453,1378\left(\mathrm{CH}_{3}\right) \mathrm{cm}^{-1}$.

UV $\left(\mathrm{CH}_{3} \mathrm{CN}\right): \lambda_{\max }(\lg \varepsilon)=234.0(3.293) \mathrm{nm}$.

${ }^{1}$ H-NMR $\left(200 \mathrm{MHz} \mathrm{CDCl}_{3}\right): \delta=1.30\left(\mathrm{~d}, J=5.9 \mathrm{~Hz}, 3 \mathrm{H}, 4-\mathrm{H}_{3}\right), 1.34-1.84(\mathrm{~m}, 6 \mathrm{H}, 2-\mathrm{H}, 1$ 'H, 5'- $\left.\mathrm{H}_{2}, 6^{\prime}-\mathrm{H}_{2}\right), 1.99\left(\mathrm{~m}_{\mathrm{c}}, 2 \mathrm{H}, 4^{\prime}-\mathrm{H}_{2}\right), 2.46$ (br. s, $\left.2 \mathrm{H}, \mathrm{OH}\right), 3.82\left(\mathrm{~m}_{\mathrm{c}}, 2 \mathrm{H}, 1-\mathrm{H}_{2}\right), 4.06-4.21$ (m, $1 \mathrm{H}, 3-\mathrm{H})$, 5.56-5.86 (m, 2 H, 2'-H, 3'-H).

${ }^{13}$ C-NMR (50.3 MHz, $\mathrm{CDCl}_{3}$ ): $\delta=22.00$ (C-4'), 22.57 (C-4), 24.90 (C-5'), 25.25 (C-6'), 34.65 (C-1'), 50.63 (C-2), 62.78 (C-1), 70.20 (C-3), 128.5, 128.6 (C-2', C-3'). 
MS (DCI, $\left.\mathrm{NH}_{3}\right): \mathrm{m} / \mathrm{z}=188$ [M+18], 205 [M+18+17], 358 [2M+18].

$\mathrm{C}_{10} \mathrm{H}_{18} \mathrm{O}_{2}$ (170.24)

\subsubsection{2-Cyclohex-2-enyl-propan-1,3-diol 228}

Gemäß AAV 6 wurde polymergebundenes Methylmalonat $143(350 \mathrm{mg})$ mit Essigsäurecyclohex-2-enylester 1755 h am Rückfluß erhitzt und gemäß AAV 8 das erhaltene Produkt von der festen Phase abgespalten. Nach säulenchromatographischer Reinigung an Kieselgel (4 g, Pentan : Essigester $=10: 1)$ erhielt man 23 mg (0.14 mmol, 40\%) eines gelben Öls.

$\mathbf{R}_{\mathbf{f}}=0.30$ (Diethylether).

IR (Film): $\widetilde{v}=3350(\mathrm{OH}), 2927(\mathrm{CH}), 1650(\mathrm{C}=\mathrm{C}) \mathrm{cm}^{-1}$.

UV $\left(\mathrm{CH}_{3} \mathrm{CN}\right): \lambda_{\max }(\lg \varepsilon)=201.5$ (4.653), 277.0 (2.574) nm.

${ }^{1} \mathbf{H}-\mathbf{N M R}\left(200 \mathrm{MHz}, \mathrm{CDCl}_{3}\right): \delta=1.30-1.87$ (m, $6 \mathrm{H}, 2-\mathrm{H}, 1^{\prime}-\mathrm{H}, 5^{\prime}-\mathrm{H}_{2}, 6$ ' $\left.-\mathrm{H}_{2}\right), 1.93-2.12(\mathrm{~m}$, 3 H, 4'- $\left.\mathrm{H}_{2}, \mathrm{OH}\right), 2.31\left(\mathrm{~m}_{\mathrm{c}}, 1 \mathrm{H}, 1^{\prime}-\mathrm{H}\right), 3.80-3.92$ (m, 4 H, 1- $\left.\mathrm{H}_{2}, 3-\mathrm{H}_{2}\right)$, 5.54-5.83 (m, $2 \mathrm{H}, 2^{\prime}-$ H, 3 '-H).

${ }^{13}$ C-NMR (75.5 MHz, $\mathrm{CDCl}_{3}$ ): $\delta=21.87$ (C-4'), 25.16 (C-5'), 26.15 (C-6'), 34.30 (C-1'), 46.54 (C-2), 64.45, 64.68 (C-1, C-3), 128.5, 129.4 (C-2', C-3').

MS (DCI, $\left.\mathrm{NH}_{3}\right): \mathrm{m} / \mathrm{z}=174$ [M+18], 191 [M+18+17], 330 [2M+18].

$\mathrm{C}_{9} \mathrm{H}_{16} \mathrm{O}_{2}$ (156.22)

\subsubsection{2-Cyclopent-2-enyl-butan-1,3-diol 229}

Gemäß AAV 6 wurde polymergebundenes Acetoacetat 138 (300 mg) mit Essigsäurecyclopent-2-enylester $\mathbf{1 7 6} 5 \mathrm{~h}$ am Rückfluß erhitzt und gemäß AAV 8 das erhaltene Produkt von der festen Phase abgespalten. Nach Reinigung an Kieselgel (5 g, Pentan : Essigester $=10$ : 1) erhielt man $16 \mathrm{mg}(0.10 \mathrm{mmol}, 49 \%)$ eines gelben Öls.

$\mathbf{R}_{\mathbf{f}}=0.38$ (Diethylether).

IR (Film): $\widetilde{v}=3372(\mathrm{OH}), 3019,2969(\mathrm{CH}), 1655(\mathrm{C}=\mathrm{C}), 1458,1377\left(\mathrm{CH}_{3}\right) \mathrm{cm}^{-1}$.

$\mathbf{U V}\left(\mathrm{CH}_{3} \mathrm{CN}\right): \lambda_{\max }(\lg \varepsilon)=235.0(3.159) \mathrm{nm}$.

${ }^{1}$ H-NMR $\left(200 \mathrm{MHz}, \mathrm{CDCl}_{3}\right): \delta=1.39\left(\mathrm{~d}, J=6.1 \mathrm{~Hz}, 3 \mathrm{H}, 4-\mathrm{H}_{3}\right), 1.57-1.97$ (m, $3 \mathrm{H}, 2-\mathrm{H}, 5$ '$\mathrm{H}_{2}$ ), 2.21-2.55 (m, $\left.5 \mathrm{H}, 1^{\prime}-\mathrm{H}, 4^{\prime}-\mathrm{H}_{2}, \mathrm{OH}\right), 3.45-4.18$ (m, $\left.3 \mathrm{H}, 1-\mathrm{H}_{2}, 3-\mathrm{H}\right), 5.42-6.01$ (m, $2 \mathrm{H}$, 2 '-H, 3'-H). 
${ }^{13}$ C-NMR (50.3 MHz, $\left.\mathrm{CDCl}_{3}\right): \delta=21.94(\mathrm{C}-4), 27.76\left(\mathrm{C}^{\prime}{ }^{\prime}\right.$ '), 32.04 (C-4'), 41.12 (C-1'), 49.47 (C-2), 58.55 (C-1), 64.53 (C-3), 131.8, 132.1 (C-2', C-3').

MS (DCI, $\left.\mathrm{NH}_{3}\right): \mathrm{m} / \mathrm{z}=174$ [M+18], $191[\mathrm{M}+18+17], 330$ [2M+18].

$\mathrm{C}_{9} \mathrm{H}_{16} \mathrm{O}_{2}$ (156.22)

\subsubsection{2-Cyclopent-2-enyl-propan-1,3-diol 230}

Gemäß AAV 6 wurde polymergebundenes Methylmalonat 143 (300 mg) mit Essigsäurecyclopent-2-enylester $\mathbf{1 7 6} 5 \mathrm{~h}$ am Rückfluß erhitzt und gemäß AAV 8 das erhaltene Produkt von der festen Phase abgespalten. Nach Chromatographie an Kieselgel (5 g, Pentan : Essigester $=10: 1)$ erhielt man $16 \mathrm{mg}(0.11 \mathrm{mmol}, 53 \%)$ eines gelben Öls.

$\mathbf{R}_{\mathbf{f}}=0.35$ (Diethylether).

IR (Film): $\widetilde{v}=3355(\mathrm{OH}), 2934,2889(\mathrm{CH}), 1652(\mathrm{C}=\mathrm{C}) \mathrm{cm}^{-1}$.

UV $\left(\mathrm{CH}_{3} \mathrm{CN}\right): \lambda_{\max }(\lg \varepsilon)=234.4(3.314) \mathrm{nm}$.

${ }^{1}$ H-NMR (200 MHz, $\left.\mathrm{CDCl}_{3}\right): \delta=1.45-1.65(\mathrm{~m}, 1 \mathrm{H}, 2-\mathrm{H}), 1.67-1.85\left(\mathrm{~m}, 1 \mathrm{H}, 1\right.$ '-H), $1.76\left(\mathrm{~m}_{\mathrm{c}}\right.$, $\left.2 \mathrm{H}, 5^{\prime}-\mathrm{H}_{2}\right), 2.17$ (br.s, $\left.2 \mathrm{H}, \mathrm{OH}\right), 2.31\left(\mathrm{~m}_{\mathrm{c}}, 2 \mathrm{H}, 4^{\prime}-\mathrm{H}_{2}\right), 2.74\left(\mathrm{~m}_{\mathrm{c}}, 1 \mathrm{H}, 1^{\prime}-\mathrm{H}\right), 3.71-3.95$ (m, 4 $\left.\mathrm{H}, 1-\mathrm{H}_{2}, 3-\mathrm{H}_{2}\right), 5.66-5.84$ (m, 2 H, 2'-H, 3'-H).

${ }^{13}$ C-NMR (50.3 MHz, $\mathrm{CDCl}_{3}$ ): $\delta=27.76\left(\mathrm{C}^{\prime} 5^{\prime}\right.$ ), 32.04 (C-4'), 44.02 (C-1'), 46.79 (C-2), 65.41, 65.53 (C-1, C-3), 131.8, 132.1 (C-2', C-3').

MS (DCI, $\left.\mathrm{NH}_{3}\right): \mathrm{m} / \mathrm{z}=160[\mathrm{M}+18], 176$ [M+18+17], 302 [2M+18].

$\mathrm{C}_{8} \mathrm{H}_{14} \mathrm{O}_{2}$ (142.19)

\subsubsection{2-(2-Methyl-cyclopent-2-enyl)-butan-1,3-diol 231}

Gemäß AAV 6 wurde polymergebundenes Acetoacetat 138 (300 mg) mit Essigsäure-2methyl-cyclopent-2-enylester $\mathbf{1 7 7} 5 \mathrm{~h}$ am Rückfluß erhitzt und gemäß AAV 8 das erhaltene Produkt von der festen Phase abgespalten. Nach säulenchromatographischer Reinigung an Kieselgel (5 g, Pentan : Essigester = $10: 1)$ wurden $7 \mathrm{mg}(0.04 \mathrm{mmol}, 19 \%)$ eines farblosen Öls erhalten.

$\mathbf{R}_{\mathbf{f}}=0.44$ (Diethylether).

IR (Film): $\widetilde{v}=3384(\mathrm{OH}), 3012,2968(\mathrm{CH}), 1651(\mathrm{C}=\mathrm{C}), 1450,1375\left(\mathrm{CH}_{3}\right) \mathrm{cm}^{-1}$.

UV $\left(\mathrm{CH}_{3} \mathrm{CN}\right): \lambda_{\max }(\lg \varepsilon)=229.0(2.847) \mathrm{nm}$. 
${ }^{1}$ H-NMR $\left(200 \mathrm{MHz}, \mathrm{C}_{6} \mathrm{D}_{6}\right): \delta=1.11\left(\mathrm{~d}, J=6.1 \mathrm{~Hz}, 3 \mathrm{H}, 4-\mathrm{H}_{3}\right)$, 1.43-1.94 (m, $3 \mathrm{H}, 2-\mathrm{H}, 5^{\prime}$ $\mathrm{H}_{2}$ ), 1.60 (s, $3 \mathrm{H}, 2^{\prime}-\mathrm{CH}_{3}$ ), 2.05-2.23 (m, 4 H, 4'- $\left.\mathrm{H}_{2}, \mathrm{OH}\right), 2.57-2.74$ (m, $1 \mathrm{H}, 1^{\prime}$ '- $\mathrm{H}$ ), 3.903.88 (m, 3 H, 1- $\left.\mathrm{H}_{2}, 3-\mathrm{H}\right), 5.20-5.39$ (m, 1 H, 2'-H).

${ }^{13}$ C-NMR (50.3 MHz, $\left.\mathrm{C}_{6} \mathrm{D}_{6}\right): \delta=22.58\left(3^{\prime}-\mathrm{CH}_{3}\right), 23.80$ (C-4), 25.95 (C-5'), 26.40 (C-1'), 31.42 (C-4'), 48.38 (C-2), 63.59 (C-1), 68.76 (C-3), 126.0 (C-2'), 140.7 (C-3').

MS (DCI, $\left.\mathrm{NH}_{3}\right): \mathrm{m} / \mathrm{z}=188$ [M+18], 205 [M+18+17].

$\mathrm{C}_{10} \mathrm{H}_{18} \mathrm{O}_{2}$ (170.24)

\subsubsection{2-(3-Methyl-cyclopent-2-enyl)-butan-1,3-diol 232}

Gemäß AAV 6 wurde polymergebundenes Acetoacetat 138 (300 mg) mit Essigsäure-3methyl-cyclopent-2-enylester $\mathbf{1 7 8} 5 \mathrm{~h}$ am Rückfluß erhitzt und gemäß AAV 8 das erhaltene Produkt von der festen Phase abgespalten. Nach Reinigung an Kieselgel (5 g, Pentan : Essigester $=10: 1)$ erhielt man $10 \mathrm{mg}(0.060 \mathrm{mmol}, 28 \%)$ eines farblosen Öls.

$\mathbf{R}_{\mathbf{f}}=0.40$ (Diethylether).

IR (Film): $\widetilde{v}=3383(\mathrm{OH}), 2966,2930(\mathrm{CH}), 1711(\mathrm{C}=\mathrm{C}), 1454,1377\left(\mathrm{CH}_{3}\right) \mathrm{cm}^{-1}$.

$\mathbf{U V}\left(\mathrm{CH}_{3} \mathrm{CN}\right): \lambda_{\max }(\lg \varepsilon)=225.0(2.777) \mathrm{nm}$.

${ }^{1}$ H-NMR $\left(200 \mathrm{MHz}, \mathrm{CDCl}_{3}\right): \delta=1.33\left(\mathrm{~d}, J=6.5 \mathrm{~Hz}, 3 \mathrm{H}, 4-\mathrm{H}_{3}\right), 1.50-2.03$ (m, $3 \mathrm{H}, 2-\mathrm{H}, 5$ '$\mathrm{H}_{2}$ ), 1.75 (s, $\left.3 \mathrm{H}, 3^{\prime}-\mathrm{CH}_{3}\right), 2.13-2.50$ (m, $\left.4 \mathrm{H}, 4^{\prime}-\mathrm{H}_{2}, \mathrm{OH}\right), 2.99$ (m $\mathrm{m}_{\mathrm{c}}, 1 \mathrm{H}, 1$ '’-H), 3.72-4.29 (m, $\left.3 \mathrm{H}, 1-\mathrm{H}_{2}, 3-\mathrm{H}\right)$, 5.65-5.85 (m, $\left.1 \mathrm{H}, 2^{\prime}-\mathrm{H}\right)$.

${ }^{13} \mathbf{C}-\mathrm{NMR}\left(50.3 \mathrm{MHz}, \mathrm{CDCl}_{3}\right): \delta=16.75(\mathrm{C}-4), 22.41\left(\mathrm{C}-3{ }^{\prime}, \mathrm{CH}_{3}\right), 24.40(\mathrm{C}-5$ ') $34.34(\mathrm{C}-$ 1'), 37.61 (C-4'), 50.53 (C-2), 61.27 (C-1), 69.17 (C-3), 126.9 (C-2'), 141.7 (C-3').

MS (DCI, $\left.\mathrm{NH}_{3}\right): \mathrm{m} / \mathrm{z}=188$ [M+18], 205 [M+18+17].

$\mathrm{C}_{10} \mathrm{H}_{18} \mathrm{O}_{2}$ (170.24)

\subsubsection{2-(3-Methyl-cyclopent-2-enyl)-propan-1,3-diol 233}

Gemäß AAV 6 wurde polymergebundenes Methylmalonat 143 (300 mg) mit Essigsäure-3methyl-cyclopent-2-enylester $\mathbf{1 7 8} 5 \mathrm{~h}$ am Rückfluß erhitzt und gemäß AAV 8 das erhaltene Produkt von der festen Phase abgespalten. Nach Reinigung an Kieselgel (5 g, Pentan : Essigester $=10: 1)$ erhielt man $5 \mathrm{mg}(0.03 \mathrm{mmol}, 14 \%)$ eines farblosen Öls.

$\mathbf{R}_{\mathbf{f}}=0.35$ (Diethylether).

IR (Film): $\widetilde{v}=3365(\mathrm{OH}), 2970,2957(\mathrm{CH}), 1642(\mathrm{C}=\mathrm{C}) \mathrm{cm}^{-1}$. 
$\mathbf{U V}\left(\mathrm{CH}_{3} \mathrm{CN}\right): \lambda_{\max }(\lg \varepsilon)=227.1(3.258) \mathrm{nm}$.

${ }^{1} \mathbf{H}$-NMR (200 MHz, $\mathrm{CDCl}_{3}$ ): $\delta=1.45-1.98$ (m, 3 H, 2-H, 5'- $\mathrm{H}_{2}$ ), 1.73 (s, $3 \mathrm{H}, 3^{\prime}-\mathrm{CH}_{3}$ ), 2.01$2.42\left(\mathrm{~m}, 4 \mathrm{H}, 4^{\prime}-\mathrm{H}_{2}, \mathrm{OH}\right), 2.67\left(\mathrm{~m}_{\mathrm{c}}, 1 \mathrm{H}, 1^{\prime}-\mathrm{H}\right), 3.66-4.01$ (m, $\left.4 \mathrm{H}, 1-\mathrm{H}_{2}, 3-\mathrm{H}_{2}\right)$, 5.56-5.70 (m, $\left.1 \mathrm{H}, 2^{\prime}-\mathrm{H}\right)$.

${ }^{13}$ C-NMR $\left(50.3 \mathrm{MHz}, \mathrm{CDCl}_{3}\right): \delta=16.75(\mathrm{C}-4), 22.41\left(\mathrm{C}-3{ }^{\prime}, \mathrm{CH}_{3}\right), 24.40(\mathrm{C}-5$ ') $34.34(\mathrm{C}-$ 1'), 37.61 (C-4'), 46.53 (C-2), 65.23 (C-1, C-3), 126.9 (C-2'), 141.7 (C-3').

MS (DCI, $\left.\mathrm{NH}_{3}\right): \mathrm{m} / \mathrm{z}=174$ [M+18], 191 [M+18+17], 330 [2M+18].

$\mathrm{C}_{9} \mathrm{H}_{16} \mathrm{O}_{2}(\mathbf{1 5 6 . 2 2})$

\subsubsection{5. (E)-2,2-Bis(3-phenylallyl)-butan-1,3-diol 239}

Gemäß AAV 6 wurde polymergebundenes Acetoacetat 138 (300 mg) mit (E)-Essigsäure-3phenylallylester $1604 \mathrm{~h}$ am Rückfluß erhitzt und gemäß AAV 8 das erhaltene Produkt von der festen Phase abgespalten. Nach säulenchromatographischer Reinigung an Kieselgel (5 g, Pentan : Essigester $=5: 1)$ wurden $35 \mathrm{mg}(0.11 \mathrm{mmol}, 52 \%)$ einer klaren Flüssigkeit erhalten.

$\mathbf{R}_{\mathbf{f}}=0.60$ (Diethylether).

IR (Film): $\widetilde{v}=3372(\mathrm{OH}), 3034,2970(\mathrm{CH}), 1651(\mathrm{C}=\mathrm{C}), 1448,1378\left(\mathrm{CH}_{3}\right) \mathrm{cm}^{-1}$.

UV $\left(\mathrm{CH}_{3} \mathrm{CN}\right): \lambda_{\max }(\lg \varepsilon)=202.5$ (4.510), 252.0 (4.409), 283.5 (3.472), 293.0 (3.255) nm.

${ }^{1}$ H-NMR $\left(200 \mathrm{MHz}, \mathrm{CDCl}_{3}\right): \delta=1.31\left(\mathrm{~d}, J=6.3 \mathrm{~Hz}, 3 \mathrm{H}, 4-\mathrm{H}_{3}\right), 2.09(\mathrm{dd}, J=14.2,7.5 \mathrm{~Hz}$, $\left.1 \mathrm{H}, 1^{\prime}-\mathrm{H}_{\mathrm{a}}\right), 2.37$ (dd, $J=14.2,7.5 \mathrm{~Hz}, 1 \mathrm{H}, 1$ ' $-\mathrm{H}_{\mathrm{b}}$ ), 2.46 (d, $J=7.5 \mathrm{~Hz}, 2 \mathrm{H}, 1$ ' '- $\left.\mathrm{H}_{2}\right), 2.86$ (br. s, $2 \mathrm{H}, \mathrm{OH}), 3.65$ (d, $\left.J=10.7 \mathrm{~Hz}, 1 \mathrm{H}, 1-\mathrm{H}_{\mathrm{a}}\right), 3.83$ (d, $\left.J=10.7 \mathrm{~Hz}, 1 \mathrm{H}, 1-\mathrm{H}_{\mathrm{b}}\right), 3.97$ (q, $J$ $=6.3 \mathrm{~Hz}, 1 \mathrm{H}, 3-\mathrm{H}), 6.25\left(\mathrm{dt}, J=15.5,7.5 \mathrm{~Hz}, 1 \mathrm{H}, 2^{\prime}-\mathrm{H}\right), 6.33(\mathrm{dt}, J=15.5,7.5 \mathrm{~Hz}, 1 \mathrm{H}$, 2' '-H), 6.46 (d, $J=15.5 \mathrm{~Hz}, 1 \mathrm{H}, 3$ '-H), 6.48 (d, $J=15.5 \mathrm{~Hz}, 1 \mathrm{H}, 3$ '’-H), 7.15-7.42 (m, 10 $\mathrm{H}, \mathrm{Ph}-\mathrm{H})$.

${ }^{13}$ C-NMR (75.5 MHz, $\left.\mathrm{CDCl}_{3}\right): \delta=18.09$ (C-4), 34.63, 36.19 (C-1', C-1'’), 45.04 (C-2), 67.65 (C-1), 73.62 (C-3), 125.8, 126.0 (C-3', C-3' '), 126.0 (C-5', C-9', C-5', C-9'’), 127.1, 127.2 (C-2', C-2''), 128.5, 128.5 (C-7', C-7''), 133.1 (C-6', C-8', C-6', C-8', ), 137.3, 137.3 (C-4', C-4'').

MS (DCI, $\left.\mathrm{NH}_{3}\right): \mathrm{m} / \mathrm{z}=338$ [M+18], 355 [M+18+17], 658 [2M+18].

$\mathrm{C}_{22} \mathrm{H}_{26} \mathrm{O}_{2}$ (320.44) 


\subsubsection{6. (E)-2,2-Bis(3-phenylallyl)-propan-1,3-diol 240}

Gemäß AAV 6 wurde polymergebundenes Methylmalonat 143 (300 mg) mit (E)-Essigsäure-3phenylallylester $1605 \mathrm{~h}$ am Rückfluß erhitzt und gemäß AAV 8 das erhaltene Produkt von der festen Phase abgespalten. Nach Reinigung an Kieselgel (5 g, Pentan : Essigester $=5: 1)$ erhielt man $37 \mathrm{mg}$ (0.12 mmol, 57\%) eines farblosen Öls.

$\mathbf{R}_{\mathbf{f}}=0.55$ (Diethylether).

IR (Film): $\widetilde{v}=3384(\mathrm{OH}), 3026,2925$ (CH), 1651 (C=C), 744, 693 (monosubst. Aromat) $\mathrm{cm}^{-1}$.

UV $\left(\mathrm{CH}_{3} \mathrm{CN}\right): \lambda_{\max }(\lg \varepsilon)=202.0(4.550), 251.5$ (4.420), 283.5 (3.481), $293.0(3.273) \mathrm{nm}$.

${ }^{1} \mathbf{H}$-NMR $\left(200 \mathrm{MHz}, \mathrm{CDCl}_{3}\right): \delta=2.28\left(\mathrm{~d}, J=7.4 \mathrm{~Hz}, 4 \mathrm{H}, 1\right.$ ' $-\mathrm{H}_{2}, 1$ '’-$\left.-\mathrm{H}_{2}\right), 2.56$ (br. s, $2 \mathrm{H}$, $\mathrm{OH}), 3.68$ (s, $4 \mathrm{H}, 1-\mathrm{H}_{2}, 3-\mathrm{H}_{2}$ ), 6.27 (dt, $J=15.7,7.4 \mathrm{~Hz}, 2 \mathrm{H}, 2$ '-H, 2'’-H), 6.48 (d, $J=15.7$, 2 H, 3'-H, 3' '-H), 7.15-7.41 (m, 10 H, Ph-H).

${ }^{13}$ C-NMR (50.3 MHz, CDCl 3$): \delta=35.38$ (C-1', C-1' ') 43.34 (C-2), 68.23 (C-1, C-3), 125.4 (C-2', C-2'’), 126.0 (C-5', C-9', C-5', C-9'’), 127.1 (C-3', C-3'’), 128.5 (C-6', C-8', C-6', C-8'’), 133.1 (C-7', C-7'’), 137.2 (C-4', C-4' ').

MS (DCI, $\left.\mathrm{NH}_{3}\right): \mathrm{m} / \mathrm{z}=326[\mathrm{M}+18]$.

$\mathrm{C}_{21} \mathrm{H}_{24} \mathrm{O}_{2}$ (308.41)

\subsubsection{7. (E)-2,2-Bis(3-(4-dimethylaminophenyl)-allyl)-butan-1,3-diol 241}

Gemäß AAV 6 wurde polymergebundenes Acetoacetat 138 (300 mg) mit (E)-Essigsäure-3-(4dimethyaminophenyl)-allylester $1795 \mathrm{~h}$ am Rückfluß erhitzt und gemäß AAV 8 das erhaltene Produkt von der festen Phase abgespalten. Nach Reinigung an Kieselgel (5 g, Pentan : Essigester $=5: 1)$ wurden $32 \mathrm{mg}(0.071 \mathrm{mmol}, 34 \%)$ eines klaren Öls erhalten.

$\mathbf{R}_{\mathbf{f}}=0.65$ (Diethylether) .

IR (Film): $\widetilde{v}=3376(\mathrm{OH}), 2924,2887(\mathrm{CH}), 1610(\mathrm{C}=\mathrm{C}), 1479,1352\left(\mathrm{CH}_{3}\right) \mathrm{cm}^{-1}$.

UV $\left(\mathrm{CH}_{3} \mathrm{CN}\right): \lambda_{\max }(\lg \varepsilon)=200.5$ (4.559), 295.5 ( 1.441) nm.

${ }^{1} \mathbf{H}$-NMR $\left(200 \mathrm{MHz}, \mathrm{CDCl}_{3}\right): \delta=1.31\left(\mathrm{~d}, J=6.4 \mathrm{~Hz}, 3 \mathrm{H}, 4-\mathrm{H}_{3}\right), 2.06(\mathrm{dd}, J=13.8,6.9 \mathrm{~Hz}$, $1 \mathrm{H}, 1$ '- $-\mathrm{H}_{\mathrm{a}}$ ), 2.34 (dd, $J=13.8,6.9 \mathrm{~Hz}, 1 \mathrm{H}, 1$ '- $\mathrm{H}_{\mathrm{b}}$ ), 2.43 (d, $J=6.9 \mathrm{~Hz}, 2 \mathrm{H}, 1$ '’- $\mathrm{H}_{2}$ ), 2.76$2.90(\mathrm{~m}, 2 \mathrm{H}, \mathrm{OH}), 2.95\left(\mathrm{~s}, 12 \mathrm{H}, \mathrm{NCH}_{3}\right), 3.64\left(\mathrm{dd}, J=11,5.2 \mathrm{~Hz}, 1 \mathrm{H}, 1-\mathrm{H}_{\mathrm{a}}\right), 3.82(\mathrm{dd}, J=$ 11, $\left.5.2 \mathrm{~Hz}, 1 \mathrm{H}, 1-\mathrm{H}_{\mathrm{b}}\right), 3.98\left(\mathrm{~m}_{\mathrm{c}}, 1 \mathrm{H}, 3-\mathrm{H}\right), 6.08$ (dt, $\left.J=15.4,6.4 \mathrm{~Hz}, 1 \mathrm{H}, 2^{\prime}-\mathrm{H}\right), 6.09$ (dt, $J$ = 15.4, 6.4 Hz, $1 \mathrm{H}, 2$ ''-H), 6.38 (d, $\left.J=15.4 \mathrm{~Hz}, 1 \mathrm{H}, 3^{\prime}-\mathrm{H}\right), 6.40$ (d, $J=15.4 \mathrm{~Hz}, 1 \mathrm{H}, 3$ ''- 
H), 6.69 (d, J = 8.0 Hz, 4 H, 6'-H, 8'-H, 6' '-H, 8' '-H), 7.27 (d, J = 8.0 Hz, 4 H, 5'-H, 9'-H, 5 ',-H, 9'-H).

${ }^{13}$ C-NMR (75.5 MHz, $\left.\mathrm{CDCl}_{3}\right): \delta=18.10$ (C-4), 34.38, 36.25 (C-1', C-1'”), 43.48, 43.51 $\left(\mathrm{NCH}_{3}\right), 45.12$ (C-2), 67.51 (C-1), 73.54 (C-3), 113.1, 113.2 (C-6', C-8', C-6', C-8' '), 124.3 (C-4', C-4'’), 125.7, 126.0 (C-3', C-3' '), 127.0, 127.1 (C-5', C-9', C-5', C-9'’), 127.2, 127.3 (C-2', C-2'’), 137.3, 143.5, 143.7 (C-7', C-7'’).

MS (DCI, $\left.\mathrm{NH}_{3}\right): \mathrm{m} / \mathrm{z}=426$ [M+18], $443[\mathrm{M}+18+17]$.

$\mathrm{C}_{26} \mathrm{H}_{36} \mathrm{~N}_{2} \mathrm{O}_{2}$ (408.57)

\subsubsection{8. (E)-2,2-Bis(3-(4-dimethylaminophenyl)-allyl)-propan-1,3-diol 242}

Gemäß AAV 6 wurde polymergebundenes Methylmalonat 143 (300 mg) mit (E)-Essigsäure-3(4-dimethyaminophenyl)-allylester $1795 \mathrm{~h}$ am Rückfluß erhitzt und gemäß AAV 8 das erhaltene Produkt von der festen Phase abgespalten. Nach Reinigung an Kieselgel (5 g, Pentan : Essigester $=5: 1)$ wurden $15 \mathrm{mg}(0.037 \mathrm{mmol}, 18 \%)$ eines klaren Öls erhalten.

$\mathbf{R}_{\mathbf{f}}=0.60$ (Diethylether).

IR (Film): $\widetilde{v}=3372(\mathrm{OH}), 2989,2958(\mathrm{CH}), 1602(\mathrm{C}=\mathrm{C}) 812$ (p-subst. Aromat) $\mathrm{cm}^{-1}$.

UV $\left(\mathrm{CH}_{3} \mathrm{CN}\right): \lambda_{\max }(\lg \varepsilon)=201.7$ (4.021), 294.3 (2.044) nm.

${ }^{1}$ H-NMR (200 MHz, $\mathrm{CDCl}_{3}$ ): $\delta=2.18$ (br. s, $\left.2 \mathrm{H}, \mathrm{OH}\right), 2.25$ (d, $J=7.5 \mathrm{~Hz}, 4 \mathrm{H}, 1$ '- $\mathrm{H}_{2}, 1$ ''$\mathrm{H}_{2}$ ), 2.94 (s, $12 \mathrm{H}, \mathrm{NCH}_{3}$ ), 3.67 (s, $4 \mathrm{H}, 1-\mathrm{H}_{2}, 3-\mathrm{H}_{2}$ ), 6.07 (dt, $J=15.1,7.5 \mathrm{~Hz}, 2 \mathrm{H}, 2$ '-H, 2' H), 6.39 (d, $J=15.1 \mathrm{~Hz}, 2 \mathrm{H}, 3$ '-H, 3' -H), 6.63-6.74 (m, 4 H, 6'-H, 8'-H, 6' -H, 8' '-H), 7.23-7.32 (m, 4 H, 5'-H, 9'-H, 5'’-H, 9',-H).

${ }^{13}$ C-NMR (75.5 MHz, $\left.\mathrm{CDCl}_{3}\right): \delta=34.67\left(\mathrm{C}-1\right.$ ', C-1'’), 40.69, $40.82\left(4 * \mathrm{NCH}_{3}\right), 45.75(\mathrm{C}-2)$, 67.56, 67.60 (C-1, C-3), 112.6, 112.8 (C-6', C-8', C-6', C-8'’), 126.9, 129.9 (C-2', C-3', C2', C-3' '), 132.7 (C-5', C-9', C-5', C-9'’), 149.8 (C-7', C-7'”).

MS $\left(\mathrm{DCI}, \mathrm{NH}_{3}\right): \mathrm{m} / \mathrm{z}=395[\mathrm{M}+1], 412[\mathrm{M}+18]$.

$\mathrm{C}_{25} \mathrm{H}_{34} \mathrm{~N}_{2} \mathrm{O}_{2}$ (394.55)

\subsubsection{9. (E)-2,2-Bis(3-(3-methoxyphenyl)-allyl)-butan-1,3-diol 243}

Gemäß AAV 6 wurde polymergebundenes Acetoacetat 138 (300 mg) mit (E)-Essigsäure-3-(3methoxyphenyl)-allylester $1805 \mathrm{~h}$ am Rückfluß erhitzt und gemäß AAV 8 das erhaltene Produkt von der festen Phase abgespalten. Nach Reinigung an Kieselgel (5 g, Pentan : Essigester $=5: 1)$ wurden $17 \mathrm{mg}(0.044 \mathrm{mmol}, 23 \%)$ eines klaren Öls erhalten. 
$\mathbf{R}_{\mathbf{f}}=0.30$ (Diethylether).

IR (Film): $\widetilde{v}=3356(\mathrm{OH}), 3026,2937(\mathrm{CH}), 1599,1579(\mathrm{C}=\mathrm{C}), 1454,1377\left(\mathrm{CH}_{3}\right) \mathrm{cm}^{-1}$.

$\mathbf{U V}\left(\mathrm{CH}_{3} \mathrm{CN}\right): \lambda_{\max }(\lg \varepsilon)=216.0$ (4.934), 257.0 (4.450), 294.5 (3.804) nm.

${ }^{1} \mathbf{H}-N M R\left(200 \mathrm{MHz}, \mathrm{CDCl}_{3}\right): \delta=1.31\left(\mathrm{~d}, J=6.2 \mathrm{~Hz}, 3 \mathrm{H}, 4-\mathrm{H}_{3}\right), 2.09(\mathrm{dd}, J=13.7,7.6 \mathrm{~Hz}$, $1 \mathrm{H}, 1$ ' $-\mathrm{H}_{\mathrm{a}}$ ), 2.38 (dd, $J=13.7,7.6 \mathrm{~Hz}, 1 \mathrm{H}, 1$ '- $\left.\mathrm{H}_{\mathrm{b}}\right), 2.46$ (d, $J=7.6 \mathrm{~Hz}, 2 \mathrm{H}, 1$ '’ $\left.-\mathrm{H}_{2}\right), 2.68$ (d, $J=5.1 \mathrm{~Hz}, 1 \mathrm{H}, 3-\mathrm{OH}), 2.77$ (br. s, $1 \mathrm{H}, 1-\mathrm{OH}), 3.65$ (dd, $J=11.3,5.1 \mathrm{~Hz}, 1 \mathrm{H}, 1-\mathrm{H}_{\mathrm{a}}$ ), 3.80 (s, $6 \mathrm{H}, \mathrm{OMe}), 3.83\left(\mathrm{dd}, J=11.3,5.1 \mathrm{~Hz}, 1 \mathrm{H}, 1-\mathrm{H}_{\mathrm{b}}\right), 3.97\left(\mathrm{~m}_{\mathrm{c}}, 1 \mathrm{H}, 3-\mathrm{H}\right), 6.24(\mathrm{dt}, J=15.5$, 7.6 Hz, $1 \mathrm{H}, 2$ '-H), 6.28 (dt, $J=15.5,7.6 \mathrm{~Hz}, 1 \mathrm{H}, 2$ '’-H), 6.44 (d, $J=15.5 \mathrm{~Hz}, 1 \mathrm{H}, 3$ '-H), 6.45 (d, $J=15.5 \mathrm{~Hz}, 1 \mathrm{H}, 3$ ''-H), 6.77 (d, $J=8.2 \mathrm{~Hz}, 2 \mathrm{H}, 7^{\prime}-\mathrm{H}, 7^{\prime}$ '-H), 6.88 (s, $2 \mathrm{H}, 5$ '-H, 5' '-H), 6.95 (d, J=8.2 Hz, 2 H, 9'-H, 9' '-H), 7.22 (t, $J=8.2 \mathrm{~Hz}, 2 \mathrm{H}, 8$ '-H, 8' '-H).

${ }^{13} \mathrm{C}-\mathrm{NMR}$ (50.3 MHz, $\mathrm{CDCl}_{3}$ ): $\delta=18.01$ (C-4), 34.51 (C-1'), 36.15 (C-1'’), 44.88 (C-2), 55.22 (2*OMe), 65.16 (C-1), 73.57 (C-3), 111.5 (C-5', C-5'’), 112.6 (C-7', C-7' ') 118.7 (C9', C-9'’), 126.3 (C-3', C-3'’), 129.5 (C-2', C-2'’), 132.9 (C-8', C-8'’), 138.8 (C-4', C-4' '), 159.7 (C-6', C-6')

MS (DCI, $\left.\mathrm{NH}_{3}\right): \mathrm{m} / \mathrm{z}=400[\mathrm{M}+18], 782$ [2M+18].

$\mathrm{C}_{24} \mathrm{H}_{30} \mathrm{O}_{4}(382.49)$

\subsubsection{0. (E)-2-(3-(3-Methoxyphenyl)-allyl)-propan-1,3-diol 244}

Gemäß AAV 6 wurde polymergebundenes Methylmalonat 143 (300 mg) mit (E)-Essigsäure-3(3-methoxyphenyl)-allylester $1805 \mathrm{~h}$ am Rückfluß erhitzt und gemäß AAV 8 das erhaltene Produkt von der festen Phase abgespalten. Nach Reinigung an Kieselgel (5 g, Pentan : Essigester $=5: 1)$ wurden $11 \mathrm{mg}(0.050 \mathrm{mmol}, 24 \%)$ eines klaren Öls erhalten.

$\mathbf{R}_{\mathbf{f}}=0.25$ (Diethylether).

IR (Film): $\widetilde{v}=3374(\mathrm{OH}), 2931(\mathrm{CH}), 1598,1579(\mathrm{C}=\mathrm{C}), 1454,1383\left(\mathrm{CH}_{3}\right), 774,690(m-$ disubst. Aromat) $\mathrm{cm}^{-1}$.

UV $\left(\mathrm{CH}_{3} \mathrm{CN}\right): \lambda_{\max }(\lg \varepsilon)=215.5$ (4.346), 254.4 (4.056), 294.0 (3.376) $\mathrm{nm}$.

${ }^{1}$ H-NMR $\left(200 \mathrm{MHz}, \mathrm{CDCl}_{3}\right): \delta=1.85-2.06$ (m, $\left.3 \mathrm{H}, 2-\mathrm{H}, \mathrm{OH}\right), 2.25$ (t, $J=7.1 \mathrm{~Hz}, 2 \mathrm{H}, 1$ '$\mathrm{H}_{2}$ ), 3.67-3.94 (m, $\left.7 \mathrm{H}, 1-\mathrm{H}_{2}, 3-\mathrm{H}_{2}, \mathrm{OCH}_{3}\right), 6.19$ (dt, $J=16.0,7.1 \mathrm{~Hz}, 1 \mathrm{H}, 2$ '-H), 6.41 (d, $J$ $\left.=16.0 \mathrm{~Hz}, 1 \mathrm{H}, 3^{\prime}-\mathrm{H}\right), 6.72-6.98$ (m, 3 H, 5'-H, 7'-H, 9'-H), 7.16-7.25 (m, 1 H, 8'-H).

${ }^{13} \mathrm{C}-\mathrm{NMR}\left(50.3 \mathrm{MHz}, \mathrm{CDCl}_{3}\right): \delta=31.60\left(\mathrm{C}-1^{\prime}\right), 42.24(\mathrm{C}-2), 55.21(\mathrm{OMe}), 65.87$ (C-1, C3), 111.3 (C-2’’), 112.7 (C-5'), 118.6 (C-7), 128.2 (C-2'), 129.5 (C-3'), 131.7 (C-8'), 138.7 (C-4'), 159.7 (C-6').

MS (DCI, NH $\left.\mathrm{NH}_{3}\right): \mathrm{m} / \mathrm{z}=240[\mathrm{M}+18], 257[\mathrm{M}+18+17]$. 


\section{$\mathrm{C}_{13} \mathrm{H}_{18} \mathrm{O}_{3}$ (222.28)}

\subsubsection{1. (E)-2-(1-Ethyl-3-phenylallyl)-butan-1,3-diol 246}

Gemäß AAV 6 wurde polymergebundenes Acetoacetat 138 (300 mg) mit (E)-Essigsäure-1ethyl-3-phenylallylester 1615 h am Rückfluß erhitzt und gemäß AAV 8 das erhaltene Produkt von der festen Phase abgespalten. Nach säulenchromatographischer Reinigung an Kieselgel $(5 \mathrm{~g}$, Pentan : Essigester $=10: 1)$ erhielt man $13 \mathrm{mg}(0.056 \mathrm{mmol}, 27 \%)$ eines farblosen Öls.

$\mathbf{R}_{\mathbf{f}}=0.60$ (Diethylether).

IR (Film): $\widetilde{v}=3346(\mathrm{OH}), 3012,2968(\mathrm{CH}), 1623(\mathrm{C}=\mathrm{C}), 1448,1378\left(\mathrm{CH}_{3}\right) \mathrm{cm}^{-1}$.

UV $\left(\mathrm{CH}_{3} \mathrm{CN}\right): \lambda_{\max }(\lg \varepsilon)=202.8$ (4.421), 251.2 (4.428), 282.4 (3.671), $291.0(3.267) \mathrm{nm}$.

${ }^{1} \mathbf{H}$-NMR (200 MHz, $\left.\mathrm{C}_{6} \mathrm{D}_{6}\right): \delta=0.80-1.89$ (m, $9 \mathrm{H}, 2-\mathrm{H}, 4-\mathrm{H}_{3}, 1$ '' $-\mathrm{H}_{2}, 2$ '' $\left.-\mathrm{H}_{3}\right), 2.20-2.40$ (m, $1 \mathrm{H}, 1$ '-H), $3.72\left(\mathrm{~m}_{\mathrm{c}}, 2 \mathrm{H}, 1-\mathrm{H}_{2}\right), 3.90\left(\mathrm{~m}_{\mathrm{c}}, 1 \mathrm{H}, 3-\mathrm{H}\right), 5.93$ (dd, $J=15.6,7.3 \mathrm{~Hz}, 1 \mathrm{H}, 2$ '-H), $6.31(\mathrm{~d}, J=15.6,1 \mathrm{H}, 3$ '-H), 6.19-7.34 (m, $5 \mathrm{H}, \mathrm{Ph}-\mathrm{H})$.

${ }^{13}$ C-NMR (50.3 MHz, $\mathrm{C}_{6} \mathrm{D}_{6}$ ): $\delta=12.21$ (C-2'’), 22.07 (C-4), 24.95 (C-1'), 32.25 (C-1'), 49.63 (C-2), 67.55 (C-1), 73.58 (C-3), 125.7 (C-3'), 126.1 (C-5', C-9'), 127.1 (C-2'), 128.6 (C-7'), 133.2 (C-6', C-8'), 137.3 (C-4').

MS $\left(\mathrm{DCI}, \mathrm{NH}_{3}\right): \mathrm{m} / \mathrm{z}=235[\mathrm{M}+1], 252[\mathrm{M}+18]$.

$\mathrm{C}_{15} \mathrm{H}_{22} \mathrm{O}_{2}$ (234.33)

\subsubsection{2. (E)-2,2-Bis(3-furan-3-yl-allyl)-butan-1,3-diol 251}

Gemäß AAV 6 wurde polymergebundenes Acetoacetat 138 (300 mg) mit Essigsäure-1-furan3-yl-allylester $1905 \mathrm{~h}$ am Rückfluß erhitzt und gemäß AAV 8 das erhaltene Produkt von der festen Phase abgespalten. Nach Reinigung an Kieselgel (5 g, Pentan : Essigester $=5: 1$ ) wurden $15 \mathrm{mg}$ (0.051 mmol, 23\%) eines säureempfindlichen Öls erhalten.

$\mathbf{R}_{\mathbf{f}}=0.60$ (Diethylether).

IR (Film): $\widetilde{v}=3320(\mathrm{OH}), 3047,2978(\mathrm{CH}), 1612(\mathrm{C}=\mathrm{C}), 1451,1380\left(\mathrm{CH}_{3}\right) \mathrm{cm}^{-1}$.

UV $\left(\mathrm{CH}_{3} \mathrm{CN}\right): \lambda_{\max }(\lg \varepsilon)=222.9$ (3.546), 297.9 (3.105) $\mathrm{nm}$.

${ }^{1}$ H-NMR $\left(200 \mathrm{MHz} \mathrm{CDCl}_{3}\right): \delta=1.29\left(\mathrm{~d}, J=6.9 \mathrm{~Hz}, 3 \mathrm{H}, 4-\mathrm{H}_{3}\right), 2.03(\mathrm{dd}, J=14.5,7.3 \mathrm{~Hz}$, $1 \mathrm{H}, 1$ ' $\left.-\mathrm{H}_{\mathrm{a}}\right), 2.31\left(\mathrm{dd}, J=14.5,7.3 \mathrm{~Hz}, 1 \mathrm{H}, 1^{\prime}-\mathrm{H}_{\mathrm{b}}\right), 2.39$ (d, $J=7.2 \mathrm{~Hz}, 1$ ' ' $\left.-\mathrm{H}_{2}\right), 2.55$ (br. s, 2 $\mathrm{H}, \mathrm{OH}), 3.62$ (d, $\left.J=10.6 \mathrm{~Hz}, 1 \mathrm{H}, 1-\mathrm{H}_{\mathrm{b}}\right), 3.94$ (q, $\left.J=6.9 \mathrm{~Hz}, 3 \mathrm{H}, 3-\mathrm{H}\right), 5.96$ (dt, $J=15.7$, $\left.7.5 \mathrm{~Hz}, 1 \mathrm{H}, 2^{\prime}-\mathrm{H}\right), 6.02$ (dt, $J=15.7,7.5 \mathrm{~Hz}, 1 \mathrm{H}, 2$ '’-H), 6.31 (d, $J=15.8 \mathrm{~Hz}, 1 \mathrm{H}, 3$ '-H), 
6.33 (d, $J=15.8 \mathrm{~Hz}, 1 \mathrm{H}, 3^{\prime}$ '-H), 6.47-6.55 (m, 2 H, 8'-H, 8' '-H), 7.33-7.43 (m, 4 H, 5'-H, 7'-H, 5' '-H, 7' '-H).

${ }^{13}$ C-NMR (50.3 MHz, $\mathrm{CDCl}_{3}$ ): $\delta=18.07$ (C-4), 34.54, 36.08 (C-1', C-1'”), 44.83 (C-2), 67.61 (C-1), 73.57 (C-3), 107.4 (C-8', C-8',), 122.7 (C-3', C-3'’), 124.2 (C-4', C-4'’), 125.3 (C-2', C-2',), 139.7 (C-5', C-5''), 143.7 (C-7', C-7'”).

MS $\left(\mathrm{DCI}, \mathrm{NH}_{3}\right): \mathrm{m} / \mathrm{z}=320[\mathrm{M}+18], 337[\mathrm{M}+18+17]$.

$\mathrm{C}_{18} \mathrm{H}_{22} \mathrm{~N}_{2} \mathrm{O}_{4}$ (302.36)

\subsubsection{3. (E)-2,2-Bis(3-furan-3-yl-allyl)-propan-1,3-diol 252}

Gemäß AAV 6 wurde polymergebundenes Methylmalonat 143 (300 mg) mit Essigsäure-1furan-3-yl-allylester 1905 h am Rückfluß erhitzt und gemäß AAV 8 das erhaltene Produkt von der festen Phase abgespalten. Nach Reinigung an Kieselgel ( 5 g, Pentan : Essigester $=5: 1$ ) erhielt man $12 \mathrm{mg}(0.042 \mathrm{mmol}, 20 \%)$ eines säureempfindlichen Öls.

$\mathbf{R}_{\mathbf{f}}=0.50$ (Diethylether).

IR (Film): $\widetilde{\mathbf{v}}=3345(\mathrm{OH}), 2926(\mathrm{CH}), 1713(\mathrm{C}=\mathrm{C}) \mathrm{cm}^{-1}$.

$\mathbf{U V}\left(\mathrm{CH}_{3} \mathrm{CN}\right): \lambda_{\max }(\lg \varepsilon)=225.0$ (3.834), 297.0 (3.449) $\mathrm{nm}$.

${ }^{1}$ H-NMR $\left(200 \mathrm{MHz}, \mathrm{CDCl}_{3}\right.$ ): $\delta=1.72$ (br. s, $\left.2 \mathrm{H}, \mathrm{OH}\right), 2.23$ (d, $J=7.1 \mathrm{~Hz}, 4 \mathrm{H}, 1$ '- $\mathrm{H}_{2}, 1$ ''$\mathrm{H}_{2}$ ), 3.66 (s, $4 \mathrm{H}, 1-\mathrm{H}_{2}, 3-\mathrm{H}_{2}$ ), 5.97 (dt, $J=15.1,7.1 \mathrm{~Hz}, 2 \mathrm{H}, 2^{\prime}-\mathrm{H}, 2$ ' '-H), 6.33 (d, $J=15.1$, 2 H, 3'-H, 3' '-H), 6.52 (m, 2 H, 8'-H, 8' '-H), 7.33-7.42 (m, 4 H, 5'-H, 7'-H, 5' '-H, 7' '-H).

${ }^{13}$ C-NMR (50.3 MHz, $\mathrm{CDCl}_{3}$ ): $\delta=35.40$ (C-1', C-1'’), 43.15 (C-2), 68.53 (C-1, C-3), 107.4 (C-8', C-8',), 121.4 (C-3', C-3'’), 124.1 (C-4', C-4'”), 124.9 (C-2', C-2'’), 139.7 (C-5', C5'), 143.4 (C-7', C-7'').

MS (DCI, $\left.\mathrm{NH}_{3}\right): \mathrm{m} / \mathrm{z}=306[\mathrm{M}+18], 323[\mathrm{M}+18+17]$.

$\mathrm{C}_{17} \mathrm{H}_{20} \mathrm{O}_{4}$ (288.33)

\subsubsection{4. (E)-2,2-Bis(3-thiophen-2-yl-allyl)-butan-1,3-diol 253}

Gemäß AAV 6 wurde polymergebundenes Acetoacetat 138 (300 mg) mit Essigsäure-1thiophen-2-yl-allylester $1915 \mathrm{~h}$ am Rückfluß erhitzt und gemäß AAV 8 das erhaltene Produkt von der festen Phase abgespalten. Nach Reinigung an Kieselgel (5 g, Pentan : Essigester $=5$ : 1) erhielt man $14 \mathrm{mg}(0.042 \mathrm{mmol}, 20 \%)$ eines säureempfindlichen Öls.

$\mathbf{R}_{\mathbf{f}}=0.55$ (Diethylether). 
IR (Film): $\widetilde{v}=3417(\mathrm{OH}), 2979,2930(\mathrm{CH}), 1710,1660(\mathrm{C}=\mathrm{C}), 1437,1380\left(\mathrm{CH}_{3}\right) \mathrm{cm}^{-1}$.

UV $\left(\mathrm{CH}_{3} \mathrm{CN}\right): \lambda_{\max }(\lg \varepsilon)=237.7$ (3.907), 280.4 (3.939) $\mathrm{nm}$.

${ }^{1} \mathbf{H}-\mathbf{N M R}\left(200 \mathrm{MHz}, \mathrm{CDCl}_{3}\right): \delta=1.30\left(\mathrm{~d}, J=6.1 \mathrm{~Hz}, 3 \mathrm{H}, 4-\mathrm{H}_{3}\right), 2.06(\mathrm{~d}, J=14.1,7.9 \mathrm{~Hz}, 1$ $\left.\mathrm{H}, 1^{\prime}-\mathrm{H}_{\mathrm{a}}\right), 2.34\left(\mathrm{dd}, J=14.1,7.9 \mathrm{~Hz}, 1 \mathrm{H}, 1^{\prime}-\mathrm{H}_{\mathrm{b}}\right), 2.42$ (d, $J=6.1 \mathrm{~Hz}, 2 \mathrm{H}, 1$ ' ' $\left.-\mathrm{H}_{2}\right), 2.49-2.69$ $\left(\mathrm{m}_{\mathrm{c}}, 2 \mathrm{H}, \mathrm{OH}\right), 3.64\left(\mathrm{dd}, J=10.4,4.7 \mathrm{~Hz}, 1 \mathrm{H}, 1-\mathrm{H}_{\mathrm{a}}\right), 3.82\left(\mathrm{dd}, J=10.4,4.7 \mathrm{~Hz}, 1 \mathrm{H}, 1-\mathrm{H}_{\mathrm{b}}\right)$, $3.96\left(\mathrm{~m}_{\mathrm{c}}, 1 \mathrm{H}, 3-\mathrm{H}\right), 6.07\left(\mathrm{dt}, J=15.7,6.1 \mathrm{~Hz}, 1 \mathrm{H}, 2^{\prime}-\mathrm{H}\right), 6.08(\mathrm{dt}, J=15.7,6.1 \mathrm{~Hz}, 1 \mathrm{H}$, 2' '-H), 6.59 (d, J= 15.7 Hz, 1 H, 3'-H), 6.61 (d, $J=15.7 \mathrm{~Hz}, 1 \mathrm{H}, 3$ ' '-H), 6.85-7.00 (m, 4 H, 7'-H, 8'-H, 7' '-H, 8''-H), 7.12 (d, J=4.8 Hz, 2 H, 6'-H, 6''-H).

${ }^{13}$ C-NMR (75.5 MHz, CDCl ${ }_{3}$ ): $\delta=18.12$ (C-4), 34.47 (C-1', C-1'’), 45.09 (C-2), 67.52 (C1), 73.50 (C-3), 123.5 (C-5', C-5'’), 124.7 (C-3', C-3'’), 125.5 (C-6', C-6'’), 126.4 (C-7', C7' '), 127.3 (C-2', C-2''), 142.5 (C-4', C-4'').

MS (DCI, $\left.\mathrm{NH}_{3}\right): \mathrm{m} / \mathrm{z}=352$ [M+18], 369 [M+18+17].

$\mathrm{C}_{18} \mathrm{H}_{22} \mathrm{O}_{2} \mathrm{~S}_{2}(334.49)$

\subsubsection{5. (E)-2,2-Bis(3-thiophen-2-yl-allyl)-propan-1,3-diol 254}

Gemäß AAV 6 wurde polymergebundenes Methylmalonat 143 (300 mg) mit Essigsäure-1thiophen-2-yl-allylester $1915 \mathrm{~h}$ am Rückfluß erhitzt und gemäß AAV 8 das erhaltene Produkt von der festen Phase abgespalten. Nach säulenchromatographischer Reinigung an Kieselgel (5 g, Pentan : Essigester = $5: 1)$ erhielt man $10 \mathrm{mg}(0.029 \mathrm{mmol}, 14 \%)$ eines säureempfindlichen Öls.

$\mathbf{R}_{\mathbf{f}}=0.65$ (Diethylether).

IR (Film): $\widetilde{v}=3372(\mathrm{OH}), 2996,2970(\mathrm{CH}), 1621(\mathrm{C}=\mathrm{C}) \mathrm{cm}^{-1}$.

UV $\left(\mathrm{CH}_{3} \mathrm{CN}\right): \lambda_{\max }(\lg \varepsilon)=240.2$ (3.605), 278.4 (3.158) $\mathrm{nm}$.

${ }^{1} \mathbf{H}$-NMR $\left(200 \mathrm{MHz}, \mathrm{CDCl}_{3}\right): \delta=2.04$ (br. s, $\left.2 \mathrm{H}, \mathrm{OH}\right), 2.25$ (d, $J=7.5 \mathrm{~Hz}, 1$ '-H, 1',-H), 3.67 (s, 4 H, 1- $\mathrm{H}_{2}, 3-\mathrm{H}_{2}$ ), 6.09 (dt, $J=15.6,7.5 \mathrm{~Hz}, 2 \mathrm{H}, 2$ '-H, 2' '-H), 6.60 (d, J = $15.6 \mathrm{~Hz}, 2$ H, 3'-H, 3' '-H), 6.87-6.98 (m, 4 H, 6'-H, 7'-H, 6' '-H, 7' '-H), 7.12 (d, J = 4.7 Hz, 2 H, 5'-H, 5 ''-H).

${ }^{13}$ C-NMR (75.5 MHz, $\mathrm{CDCl}_{3}$ ): $\delta=34.47$ (C-1', C-1'’), 43.09 (C-2), 68.52 (C-1, C-3), 123.5 (C-5', C-5'’), 124.7 (C-3', C-3'’), 125.5 (C-6', C-6'’), 126.4 (C-7', C-7'’), 127.3 (C-2', C2',), 142.5 (C-4', C-4'’).

MS (DCI, $\left.\mathrm{NH}_{3}\right): \mathrm{m} / \mathrm{z}=338[\mathrm{M}+18], 355[\mathrm{M}+18+17]$.

$\mathrm{C}_{17} \mathrm{H}_{20} \mathrm{O}_{2} \mathrm{~S}_{2}(320.47)$ 


\subsection{Verwendung von Allylcarbonaten}

\subsubsection{2,2-Diallyl-butan-1,3-diol 202}

Gemäß AAV 7 wurde polymergebundenes Acetoacetat 138 (300 mg) mit Allyl-ethyl-carbonat 1965 h bei Raumtemperatur gerührt und gemäß AAV 8 das erhaltene Produkt von der festen Phase abgespalten. Nach säulenchromatographischer Reinigung an Kieselgel (5 g, Pentan : Essigester $=5: 1)$ erhielt man $24 \mathrm{mg}(0.14 \mathrm{mmol}, 68 \%)$ eines farblosen Öls.

Spektroskopische Daten siehe 4.6.1.

\subsection{2. (E)-2,2-Bis(3-phenylallyl)-butan-1,3-diol 239}

Gemäß AAV 7 wurde polymergebundenes Acetoacetat 138 (300 mg) mit (E)-3-Phenylallylethyl-carbonat 1985 h bei Raumtemperatur gerührt und gemäß AAV 8 das erhaltene Produkt von der festen Phase abgespalten. Nach säulenchromatographischer Reinigung an Kieselgel ( $5 \mathrm{~g}$, Pentan : Essigester = $5: 1)$ wurden $51 \mathrm{mg}(0.16 \mathrm{mmol}, 75 \%)$ eines farblosen Öls erhalten.

Spektroskopische Daten siehe 4.6.15.

\subsection{Verwendung von Allylchloriden}

\subsubsection{2-(2-Methyl-allyl)-propan-1,3-diol 257 und 2,2-Bis(2-methyl-allyl)-propan- 1,3-diol 212}

Polymergebundenes Methylmalonat $143(500 \mathrm{mg}$ ) wurde in trockenem THF (4 ml) gequollen und die Suspension entgast, indem $1 \mathrm{~h}$ lang Argon durch die Reaktionsmischung geleitet wurde. Bei $0{ }^{\circ} \mathrm{C}$ gab man $n$-Butyllithium ( $0.24 \mathrm{ml}, 0.37 \mathrm{mmol}, 15 \%$ in Hexan, 1 eq.) zu, ließ auf Raumtemperatur kommen und rührte für $15 \mathrm{~min}$. Anschließend wurden 3-Chlor-2-methylprop-1-en 255 (0.36 ml, 0.33 g, 3.7 mmol, 10 eq.) und Tetrakis(triphenylphosphan)palladium ( $86 \mathrm{mg}, 0.075 \mathrm{mmol}, 20 \mathrm{~mol} \%$ ) hinzugefügt und für $20 \mathrm{~h}$ bei Raumtemperatur gerührt. Es wurde abfiltriert, das Harz mit THF (20 ml), DMF (20 ml), THF (20 ml), MeOH (20 ml) und $\mathrm{CH}_{2} \mathrm{Cl}_{2}(20 \mathrm{ml})$ gewaschen und bei $55^{\circ} \mathrm{C}$ im Vakuum getrocknet. 
Gemäß AAV 8 wurde die polymergebundene Verbindung von der festen Phase abgespalten. Nach Reinigung an Kieselgel (5 g, Pentan : Diethylether $=1: 1$ ) erhielt man $14 \mathrm{mg}$ (0.10 mmol, 28\%) 257 und 7 mg (0.04 mmol, 10\%) 212 als klare Öle.

$\mathbf{R}_{\mathbf{f}}(\mathbf{2 5 7})=0.25$ (Diethylether).

$\mathbf{R}_{\mathbf{f}}(\mathbf{2 1 2})=0.70$ (Diethylether).

Spektroskopische Daten der Verbindung 212 siehe 4.6.5.

257:

IR (Film): $\widetilde{v}=3358(\mathrm{OH}), 3031,2977(\mathrm{CH}), 1634(\mathrm{C}=\mathrm{C}), 1451,1381\left(\mathrm{CH}_{3}\right) \mathrm{cm}^{-1}$.

$\mathbf{U V}\left(\mathrm{CH}_{3} \mathrm{CN}\right): \lambda_{\max }(\lg \varepsilon)=231.0(3.149) \mathrm{nm}$.

${ }^{1}$ H-NMR (200 MHz, $\left.\mathrm{C}_{6} \mathrm{D}_{6}\right): \delta=1.65$ (s, $\left.3 \mathrm{H}, 2^{\prime}-\mathrm{CH}_{3}\right), 1.91\left(\mathrm{~m}_{\mathrm{c}}, 3 \mathrm{H}, 2-\mathrm{H}, 1\right.$ ' $\left.-\mathrm{H}_{2}\right), 3.53$ (dd, $J$ $\left.=10.0,4.9 \mathrm{~Hz}, 2 \mathrm{H}, 1-\mathrm{H}_{\mathrm{a}}, 3-\mathrm{H}_{\mathrm{a}}\right), 3.67\left(\mathrm{dd}, J=10.0,4.9 \mathrm{~Hz}, 2 \mathrm{H}, 1-\mathrm{H}_{\mathrm{b}}, 3-\mathrm{H}_{\mathrm{b}}\right), 4.80(\mathrm{~d}, J=7.3$ $\left.\mathrm{Hz}, 2 \mathrm{H}, 3-\mathrm{H}_{2}\right)$.

${ }^{13}$ C-NMR (75.5 MHz, $\left.\mathrm{C}_{6} \mathrm{D}_{6}\right): \delta=22.24\left(2^{\prime}-\mathrm{CH}_{3}\right), 36.76(\mathrm{C}-1$ ') $), 40.18$ (C-2), 65.14 (C-1, C3), 112.2 (C-3'), 143.7 (C-2').

MS $\left(\mathrm{DCI}, \mathrm{NH}_{3}\right): \mathrm{m} / \mathrm{z}=148$ [M+18], $165[\mathrm{M}+18+17]$.

$\mathrm{C}_{7} \mathrm{H}_{14} \mathrm{O}_{2}$ (130.18)

\subsection{Verwendung von Bisallyltemplaten}

\subsubsection{2,2-Bis-(4-acetoxybut-2-enyl)-1,3-diacetoxy-butan 280}

Gemäß AAV 8 wurde der polymergebundene Allylalkohol 274 (250 mg) von der festen Phase abgespalten. Anschließend wurde gemäß AAV 3 mit Essigsäureanhydrid (4 eq.) acetyliert. Nach säulenchromatographischer Reinigung an Kieselgel (5 g, Pentan : Essigester $=10: 1)$ erhielt man $20 \mathrm{mg}(0.052 \mathrm{mmol}, 30 \%)$ eines farblosen Öls.

$\mathbf{R}_{\mathbf{f}}=0.30($ Pentan : Essigester $=10: 1)$.

IR (Film): $\widetilde{v}=2984,2946(\mathrm{CH}), 1739(\mathrm{C}=\mathrm{O}), 1659(\mathrm{C}=\mathrm{C}), 1441,1373\left(\mathrm{CH}_{3}\right) \mathrm{cm}^{-1}$.

$\mathbf{U V}\left(\mathrm{CH}_{3} \mathrm{CN}\right): \lambda_{\max }(\lg \varepsilon)=234.4$ (3.259), 257.4 (3.547) $\mathrm{nm}$.

${ }^{1} \mathbf{H}-\mathbf{N M R}\left(200 \mathrm{MHz}, \mathrm{CDCl}_{3}\right): \delta=1.21\left(\mathrm{~d}, J=6.7 \mathrm{~Hz}, 3 \mathrm{H}, 4-\mathrm{H}_{3}\right), 2.06\left(\mathrm{~s}, 12 \mathrm{H}, \mathrm{COCH}_{3}\right)$, 2.12-2.28 (m, 4 H, 1'- $\mathrm{H}_{2}, 1$ ''- $-\mathrm{H}_{2}$ ), 3.86-4.11 (m, 3 H, 1- $\left.\mathrm{H}_{2}, 3-\mathrm{H}\right), 4.52$ (d, J = 6.3 Hz, 4 H, 4'$\mathrm{H}_{2}, 4$ ''- $\mathrm{H}_{2}$ ), 5.53-5.87 (m, 4 H, 2'-H, 3'-H, 2'’-H, 3''-H). 
${ }^{13} \mathrm{C}-\mathrm{NMR}\left(50.3 \mathrm{MHz}, \mathrm{CDCl}_{3}\right): \delta=16.12(\mathrm{C}-4), 21.12,21.23,21.36,22.11\left(1-\mathrm{CO}_{2} \mathrm{CH}_{3}, 3-\right.$ $\mathrm{CO}_{2} \mathrm{CH}_{3}, 4$ '- $\mathrm{CO}_{2} \mathrm{CH}_{3}, 4$ '' $-\mathrm{CO}_{2} \mathrm{CH}_{3}$ ), 32.13, 32.29 (C-1', C-1' '), 37.89 (C-2), 68.12 (C-1), 70.55, 70.84 (C-4', C-4'’), 76.45 (C-3), 128.2, 128.3 (C-3', C-3'’), 129.4, 129.6 (C-2', C2 '’), 171.0, 171.0, 171.1, $171.2(\mathrm{C}=\mathrm{O})$.

MS (DCI, $\left.\mathrm{NH}_{3}\right): \mathrm{m} / \mathrm{z}=416[\mathrm{M}+18]$.

$\mathrm{C}_{20} \mathrm{H}_{30} \mathrm{O}_{8}$ (398.45)

\subsubsection{Synthese von polymergebundenem $(E)$-2,2-Bis(4-Acetoxy-but-2-enyl)-acetoacetat 281}

Gemäß AAV 7 wurde polymergebundenes Acetoacetat 138 (500 mg) mit (Z)-Ethyl-4hydroxybut-2-encarbonat 275 (5 eq.) 15 h bei Raumtemperatur gerührt und in Anlehnung an AAV 3 das erhaltene Produkt mit Essigsäureanhydrid (10 eq.) acetyliert.

\subsection{3. (E)-2,12-Diacetyl-7-methoxycarbonyl-tridec-4,9-diendisäure-dimethylester 285}

In Anlehnung an AAV 6 wurde polymergebundenes Allylacetat 281 (250 mg) mit Methylacetoacetat 205 (20 eq.) 5 h am Rückfluß erhitzt und gemäß AAV 9 das erhaltene Produkt von der festen Phase abgespalten. Nach säulenchromatographischer Reinigung an Kieselgel $(5 \mathrm{~g}$, Pentan : Essigester $=3: 1)$ erhielt man $35 \mathrm{mg}(0.081 \mathrm{mmol}, 50 \%)$ eines farblosen Öls.

$\mathbf{R}_{\mathbf{f}}=0.15($ Pentan : Essigester $=3: 1)$.

IR (Film): $\widetilde{v}=2955(\mathrm{CH}), 1737(\mathrm{C}=\mathrm{O}), 1437,1361\left(\mathrm{CH}_{3}\right) \mathrm{cm}^{-1}$.

UV $\left(\mathrm{CH}_{3} \mathrm{CN}\right): \lambda_{\max }(\lg \varepsilon)=233.5$ (3.914), 260.0 (3.609) $\mathrm{nm}$.

${ }^{1}$ H-NMR $\left(200 \mathrm{MHz}_{\mathrm{CDCl}}\right): \delta=2.07-2.46\left(\mathrm{~m}, 5 \mathrm{H}, 6-\mathrm{H}_{2}, 7-\mathrm{H}, 8-\mathrm{H}_{2}\right), 2.21\left(\mathrm{~s}, 6 \mathrm{H}, \mathrm{COCH}_{3}\right)$, $2.54\left(\mathrm{~m}_{\mathrm{c}}, 4 \mathrm{H}, 3-\mathrm{H}_{2}, 11-\mathrm{H}_{2}\right), 3.47(\mathrm{t}, J=7.4 \mathrm{~Hz}, 2 \mathrm{H}, 2-\mathrm{H}, 12-\mathrm{H}), 3.64\left(\mathrm{~s}, 3 \mathrm{H}, 2-\mathrm{CO}_{2} \mathrm{CH}_{3}\right)$, 3.73 (s, $\left.6 \mathrm{H}, 1-\mathrm{OCH}_{3}, 13-\mathrm{OCH}_{3}\right), 5.31-5.45$ (m, $\left.4 \mathrm{H}, 4-\mathrm{H}, 5-\mathrm{H}, 9-\mathrm{H}, 10-\mathrm{H}\right)$.

${ }^{13}$ C-NMR $\left(50.3 \mathrm{MHz}, \mathrm{CDCl}_{3}\right): \delta=29.10,29.14\left(\mathrm{COCH}_{3}\right), 31.10,31.14(\mathrm{C}-3, \mathrm{C}-11), 34.33$, 34.49 (C-6, C-8), 45.33 (C-7), 51.47, $52.43\left(\mathrm{OCH}_{3}\right), 128.11,128.2$ (C-5, C-9), 129.9, 129.9 (C-4, C-10), 169.7 (C-1, C-13), $175.1(7-\mathrm{C}=\mathrm{O}), 202.4$ (2-C=O, 12-C=O).

MS (DCI, $\left.\mathrm{NH}_{3}\right): \mathrm{m} / \mathrm{z}=428[\mathrm{M}+18]$.

$\mathrm{C}_{21} \mathrm{H}_{30} \mathrm{O}_{8}$ (410.45) 


\subsection{4. (E)-2,7,12-Trismethoxycarbonyl-tridec-4,9-diendisäure-dimethylester 286}

In Anlehnung an AAV 6 wurde polymergebundenes Allylacetat 281 (250 mg) mit Dimethylmalonat 282 (20 eq.) $5 \mathrm{~h}$ am Rückfluß erhitzt und gemäß AAV 9 das erhaltene Produkt von der festen Phase abgespalten. Nach säulenchromatographischer Reinigung an Kieselgel (5 g, Pentan : Essigester $=3: 1)$ erhielt man $31 \mathrm{mg}(0.070 \mathrm{mmol}, 40 \%)$ eines farblosen Öls.

$\mathbf{R}_{\mathbf{f}}=0.10($ Pentan : Essigester $=3: 1)$.

IR (Film): $\widetilde{v}=2955(\mathrm{CH}), 1732(\mathrm{C}=\mathrm{O}), 1438,1341\left(\mathrm{CH}_{3}\right) \mathrm{cm}^{-1}$.

$\mathbf{U V}\left(\mathrm{CH}_{3} \mathrm{CN}\right): \lambda_{\max }(\lg \varepsilon)=237.4(3.148), 2.59 .4(3.015) \mathrm{nm}$.

${ }^{1} \mathbf{H}-\mathbf{N M R}\left(200 \mathrm{MHz}, \mathrm{CDCl}_{3}\right): \delta=2.11-2.53\left(\mathrm{~m}, 5 \mathrm{H}, 6-\mathrm{H}_{2}, 7-\mathrm{H}, 8-\mathrm{H}_{2}\right), 2.58\left(\mathrm{~m}_{\mathrm{c}}, 4 \mathrm{H}, 3-\mathrm{H}_{2}\right.$, $\left.11-\mathrm{H}_{2}\right), 3.40$ (t, $\left.J=7.2 \mathrm{~Hz}, 2 \mathrm{H}, 2-\mathrm{H}, 12-\mathrm{H}\right), 3.65\left(\mathrm{~s}, 3 \mathrm{H}, 7-\mathrm{CO}_{2} \mathrm{CH}_{3}\right), 3.73$ (s, $12 \mathrm{H}, 1-\mathrm{OCH}_{3}$, 2- $\mathrm{CO}_{2} \mathrm{CH}_{3}, 12-\mathrm{CO}_{2} \mathrm{CH}_{3}, 13-\mathrm{OCH}_{3}$ ), 5.39-5.48 (m, $\left.4 \mathrm{H}, 4-\mathrm{H}, 5-\mathrm{H}, 9-\mathrm{H}, 10-\mathrm{H}\right)$.

${ }^{13}$ C-NMR (50.3 MHz, $\mathrm{CDCl}_{3}$ ): $\delta=31.80$ (C-3, C-11), 34.28, 34.47 (C-6, C-8), 45.27 (C-7), 51.44, 51.75 (C-2, C-12), 52.49, $52.56\left(\mathrm{OCH}_{3}\right), 126.9,128.0$ (C-5, C-9), 130.1, 130.2 (C-4, $\mathrm{C}-10), 169.2$ (C-1, C-13, 2-C=O, 12-C=O), $175.0(7-\mathrm{C}=\mathrm{O})$.

MS (DCI, $\left.\mathrm{NH}_{3}\right): \mathrm{m} / \mathrm{z}=460[\mathrm{M}+18]$.

$\mathrm{C}_{21} \mathrm{H}_{30} \mathrm{O}_{10}$ (442.45)

\subsection{5. (E)-6-Hydroxy-2-(4-hydroxybut-2-enyl)-hexen-4-ensäure-methylester 288}

Gemäß AAV 9 wurde der polymergebundene Allylalkohol 281 (200 mg) von der festen Phase abgespalten. Nach säulenchromatographischer Reinigung an Kieselgel (5 g, Pentan : Essigester $=3$ : 1) erhielt man $14 \mathrm{mg}(0.067 \mathrm{mmol}, 48 \%)$ eines farblosen Öls.

$\mathbf{R}_{\mathbf{f}}=0.05($ Pentan : Essigester $=1: 1)$.

IR (Film): $\widetilde{v}=3358(\mathrm{OH}), 3010,2980(\mathrm{CH}), 1739(\mathrm{C}=\mathrm{O}), 1638(\mathrm{C}=\mathrm{C}) \mathrm{cm}^{-1}$.

UV $\left(\mathrm{CH}_{3} \mathrm{CN}\right): \lambda_{\max }(\lg \varepsilon)=224.0(2.687) \mathrm{nm}$.

${ }^{1}$ H-NMR (200 MHz, $\mathrm{CDCl}_{3}$ ): $\delta=2.18-2.57$ (m, $5 \mathrm{H}, 2-\mathrm{H}, 3-\mathrm{H}_{2}, 1$ ' - $\mathrm{H}_{2}$ ), 3.35 (br. s, $2 \mathrm{H}, \mathrm{OH}$ ), 3.65 (s, $\left.3 \mathrm{H}, \mathrm{OCH}_{3}\right), 4.06$ (d, J = 4.1 Hz, 4 H, 6- $\left.\mathrm{H}_{2}, 4-\mathrm{H}_{2}\right), 5.58-5.69$ (m, $4 \mathrm{H}, 4-\mathrm{H}, 5-\mathrm{H}, 2$ '-H, 3 '-H).

${ }^{13} \mathbf{C}-\mathrm{NMR}\left(50.3 \mathrm{MHz}, \mathrm{CDCl}_{3}\right): \delta=34.16\left(\mathrm{C}-3{ }^{\prime}, \mathrm{C}-1^{\prime}\right), 45.07(\mathrm{C}-2), 51.63\left(\mathrm{OCH}_{3}\right), 63.24(\mathrm{C}-$ 6, C-4'), 128.8 (C-5, C-3'), 131.5 (C-4, C-2'), 175.3 (C=O).

MS $\left(\mathrm{DCI}, \mathrm{NH}_{3}\right): \mathrm{m} / \mathrm{z}=232[\mathrm{M}+18], 446[2 \mathrm{M}+18]$.

$\mathrm{C}_{11} \mathrm{H}_{18} \mathrm{O}_{4}$ (214.25) 


\subsection{Variation der Abspaltungsbedingungen}

\subsubsection{2,2-Diallyl-malonsäure-dimethylester 262}

Gemäß AAV 6 wurde polymergebundenes Methylmalonat 143 (300 mg) mit Essigsäureallylester $1995 \mathrm{~h}$ am Rückfluß erhitzt und gemäß AAV 9 das erhaltene Produkt von der festen Phase abgespalten. Nach säulenchromatographischer Reinigung an Kieselgel (5 g, Pentan : Essigester $=25: 1)$ erhielt man $18 \mathrm{mg}(0.086 \mathrm{mmol}, 41 \%)$ eines säureempfindlichen Öls.

$\mathbf{R}_{\mathbf{f}}=0.50$ (Diethylether).

IR (Film): $\widetilde{v}=2970,2961(\mathrm{CH}), 1732(\mathrm{C}=\mathrm{O}), 1648(\mathrm{C}=\mathrm{C}), 1445,1380\left(\mathrm{CH}_{3}\right) \mathrm{cm}^{-1}$.

$\mathbf{U V}\left(\mathrm{CH}_{3} \mathrm{CN}\right): \lambda_{\max }(\lg \varepsilon)=226.0(3.843) \mathrm{nm}$.

${ }^{1}$ H-NMR $\left(200 \mathrm{MHz}, \mathrm{CDCl}_{3}\right): \delta=2.64\left(\mathrm{~d}, J=7.5 \mathrm{~Hz}, 4 \mathrm{H}, 1^{\prime}-\mathrm{H}_{2}, 1\right.$ ''- $\left.-\mathrm{H}_{2}\right), 3.72(\mathrm{~s}, 6 \mathrm{H}$, OMe), 5.07 (d, $J=11.7 \mathrm{~Hz}, 2 \mathrm{H}, 3^{\prime}-\mathrm{H}_{\mathrm{a}}, 3$ '' $-\mathrm{H}_{\mathrm{a}}$ ), 5.14 (d, $J=15.3 \mathrm{~Hz}, 2 \mathrm{H}, 3$ ' $-\mathrm{H}_{\mathrm{b}}, 3$ ''- $-\mathrm{H}_{\mathrm{b}}$ ), 5.64 (ddd, $J=15.3,11.7,7.5 \mathrm{~Hz}, 2 \mathrm{H}, 2$ '-H, 2' '-H).

${ }^{13}$ C-NMR (50.3 MHz, $\left.\mathrm{CDCl}_{3}\right): \delta=36.94$ (C-1', C-1'’), 52.38 (2*OMe), $57.64(\mathrm{C}-2), 119.2$ (C-3', C-3'), 132.2 (C-2', C-2'’), $171.1(\mathrm{C}=\mathrm{O})$.

MS (DCI, $\left.\mathrm{NH}_{3}\right): \mathrm{m} / \mathrm{z}=213[\mathrm{M}+1], 230$ [M+18], 247 [M+18+17].

$\mathrm{C}_{11} \mathrm{H}_{16} \mathrm{O}_{4}(\mathbf{2 1 2 . 2 4})$

\subsection{2. (E)-5-Phenyl-2-(3-phenylallyl)-pent-4-ensäure-methylester 263}

Gemäß AAV 6 wurde polymergebundenes Acetoacetat 138 (300 mg) mit (E)-Essigsäure-3phenylallylester $1605 \mathrm{~h}$ am Rückfluß erhitzt und gemäß AAV 9 das erhaltene Produkt von der festen Phase abgespalten. Nach säulenchromatographischer Reinigung an Kieselgel (5 g, Pen$\tan :$ Essigester $=25: 1)$ erhielt $\operatorname{man} 34 \mathrm{mg}(0.11 \mathrm{mmol}, 53 \%)$ eines farblosen Öls.

$\mathbf{R}_{\mathbf{f}}=0.55($ Pentan : Essigester $=5: 1)$.

IR (Film): $\widetilde{v}=3029,2953(\mathrm{CH}), 1728(\mathrm{C}=\mathrm{O}), 1651(\mathrm{C}=\mathrm{C}) \mathrm{cm}^{-1}$.

UV $\left(\mathrm{CH}_{3} \mathrm{CN}\right): \lambda_{\max }(\lg \varepsilon)=249.0$ (3.815), 282.5 (2.772), 292.0 (2.924) nm.

${ }^{1} \mathbf{H}-\mathbf{N M R}\left(200 \mathrm{MHz}, \mathrm{CDCl}_{3}\right): \delta=2.37-2.75\left(\mathrm{~m}, 5 \mathrm{H}, 2-\mathrm{H}, 3-\mathrm{H}_{2}, 1\right.$ '- $\left.\mathrm{H}_{2}\right), 3.68$ (s, $\left.3 \mathrm{H}, \mathrm{OMe}\right)$, 6.08 (dt, $\left.J=15.5,6.9 \mathrm{~Hz}, 2 \mathrm{H}, 4-\mathrm{H}, 2^{\prime}-\mathrm{H}\right), 6.45$ (d, $J=15.5 \mathrm{~Hz}, 2 \mathrm{H}, 5-\mathrm{H}, 3$ '-H), 7.16-7.40 (m, $10 \mathrm{H}, \mathrm{Ph}-\mathrm{H})$.

${ }^{13}$ C-NMR $\left(50.3 \mathrm{MHz}, \mathrm{CDCl}_{3}\right): \delta=35.51\left(\mathrm{C}-3, \mathrm{C}-1\right.$ ') $45.80(\mathrm{C}-2), 51.09\left(\mathrm{OCH}_{3}\right), 126.5(\mathrm{C}-$ 7, C-11, C-5', C-9'), 127.1 (C-4, C-2'), 128.7 (C-8, C-10, C-6', C-8'), 129.0 (C-9, C-7'), 132.7 (C-5, C-3'), 137.8 (C-6, C-4'), 174.7 (C=O). 
MS (DCI, $\left.\mathrm{NH}_{3}\right): \mathrm{m} / \mathrm{z}=307[\mathrm{M}+1], 324$ [M+18], $341[\mathrm{M}+18+17]$.

$\mathrm{C}_{21} \mathrm{H}_{22} \mathrm{O}_{2}$ (306.39)

\subsubsection{2-Allyl-pent-4-ensäure 264}

Gemäß AAV 6 wurde polymergebundenes Acetoacetat 138 (200 mg) mit Essigsäureallylester $1995 \mathrm{~h}$ am Rückfluß erhitzt und gemäß AAV 9 das erhaltene Produkt unter Zusatz eines Tropfen Wassers von der festen Phase abgespalten. Man erhielt $11 \mathrm{mg}$ (0.074 mmol, 56\%) eines farblosen Öls.

$\mathbf{R}_{\mathbf{f}}=0.55$ (Pentan : Essigester $\left.=5: 1\right)$.

IR (Film): $\widetilde{v}=3080,2981(\mathrm{CH}), 1710(\mathrm{C}=\mathrm{O}), 1643(\mathrm{C}=\mathrm{C}) \mathrm{cm}^{-1}$.

$\mathbf{U V}\left(\mathrm{CH}_{3} \mathrm{CN}\right): \lambda_{\max }(\lg \varepsilon)=234.3(3.181) \mathrm{nm}$.

${ }^{1} \mathbf{H}$-NMR $\left(200 \mathrm{MHz}, \mathrm{CDCl}_{3}\right): \delta=2.31-2.65\left(\mathrm{~m}, 6 \mathrm{H}, 2-\mathrm{H}, 3-\mathrm{H}_{2}, 1{ }^{\prime}-\mathrm{H}_{2}, \mathrm{OH}\right), 5.07(\mathrm{~d}, J=11.3$ $\mathrm{Hz}, 2 \mathrm{H}, 5-\mathrm{H}_{\mathrm{a}}, 3$ '- $\left.\mathrm{H}_{\mathrm{a}}\right), 5.11\left(\mathrm{~d}, J=15.4 \mathrm{~Hz}, 2 \mathrm{H}, 5-\mathrm{H}_{\mathrm{b}}, 3\right.$ ' $\left.-\mathrm{H}_{\mathrm{b}}\right), 5.80$ (ddt, $J=15.4,11.3,6.5$ $\mathrm{Hz}, 2 \mathrm{H}, 4-\mathrm{H}, 2$ '-H).

${ }^{13}$ C-NMR (50.3 MHz, CDCl 3 ): $\delta=35.14$ (C-3, C-1'), 45.10 (C-2), 118.3 (C-5, C-3'), 139.1 (C-4, C-2'), $178.4(\mathrm{C}=\mathrm{O})$.

MS (70 eV, EI): m/z (\%) = $140(10)\left[\mathrm{M}^{+}\right], 99$ (95) [M+-Allyl], 95 (100) $\left[\mathrm{M}^{+}-\mathrm{CO}_{2} \mathrm{H}\right], 41$ (90) [Allyl].

\section{$\mathrm{C}_{8} \mathrm{H}_{12} \mathrm{O}_{2}(\mathbf{1 4 0 . 1 8 )}$}

\subsection{4. $N, N$ '-Dibenzylmalonamid 266}

Polymergebundenes Methylmalonat 143 (300 mg, 1 eq.) wurde in trockenem THF (5 ml) gequollen, mit Benzylamin (0.452 g, 4.2 mmol, 20 eq.) versetzt und für $14 \mathrm{~h}$ am Rückfluß erhitzt. Nach dem Abkühlen wurde das Harz filtriert und mit $\mathrm{MeOH}(20 \mathrm{ml})$ und $\mathrm{CH}_{2} \mathrm{Cl}_{2}$ (20 ml) gewaschen. Die aus der Waschlösung auskristallisierende Zielverbindung wurde abfiltriert, mit $\mathrm{Et}_{2} \mathrm{O}(5 \mathrm{ml})$ gewaschen und bei $55^{\circ} \mathrm{C} 1 \mathrm{~h}$ im Vakuum getrocknet. Man erhielt $29 \mathrm{mg}$ (0.10 mmol, 48\%) 266 als weißes Pulver.

$\mathbf{R}_{\mathbf{f}}=0.15($ Pentan $:$ Essigester $=3: 1)$.

${ }^{1} \mathbf{H}-\mathbf{N M R}\left(200 \mathrm{MHz}, \mathrm{CDCl}_{3}\right.$ ): $\delta=2.07$ (br. s, $\left.2 \mathrm{H}, \mathrm{NH}\right), 3.24$ (s, $2 \mathrm{H}, 2-\mathrm{H}_{2}$ ), 4.45 (s, $4 \mathrm{H}, 1$ '$\mathrm{H}_{2}, 1$ ''- $\left.\mathrm{H}_{2}\right), 7.13-7.41$ (m, $\left.10 \mathrm{H}, \mathrm{Ph}-\mathrm{H}\right)$.

$\mathrm{C}_{17} \mathrm{H}_{18} \mathrm{O}_{2} \mathrm{~N}_{2}$ (282.33) 


\subsection{Heck-Reaktionen an der festen Phase}

\subsection{1. (E)-2,2-Bis(3-phenylallyl)-butan-1,3-diol 239}

Polymergebundenes 2,2-Diallylacetoacetat 200 (300 mg, 1 eq.) wurde in trockenem $\mathrm{MeCN}$ (4 ml) gequollen und die Suspension entgast, indem $1 \mathrm{~h}$ lang Argon durch die Reaktionsmischung geleitet wurde. Man fügte Iodbenzol ( $0.25 \mathrm{ml}, 0.45 \mathrm{~g}, 2.2 \mathrm{mmol}, 10 \mathrm{eq}$.), Triethylamin $(0.091 \mathrm{ml}, \quad 0.066 \mathrm{~g}, \quad 0.66 \mathrm{mmol}, 3 \mathrm{eq}$.$) und Palladiumacetat (10 \mathrm{mg}, 0.045 \mathrm{mmol}$, $20 \mathrm{~mol} \%$ ) hinzu und erhitzte für $7 \mathrm{~h}$ auf $80^{\circ} \mathrm{C}$. Anschließend wurde abfiltriert, das Harz mit DMF (20 ml), THF (20 ml), MeOH $(20 \mathrm{ml})$ und $\mathrm{CH}_{2} \mathrm{Cl}_{2}(20 \mathrm{ml})$ gewaschen und bei $55^{\circ} \mathrm{C} \mathrm{im}$ Vakuum getrocknet.

Gemäß AAV 8 wurde die Zielverbindung von der festen Phase abgespalten und man erhielt nach säulenchromatographischer Reinigung an Kieselgel ( $4 \mathrm{~g}$, Pentan : Essigester $=5: 1$ ) $43 \mathrm{mg}(0.13 \mathrm{mmol}, 64 \%) \mathbf{2 3 9}$ als farbloses Öl.

Spektroskopische Daten siehe 4.6.15.

\subsection{2. (E)-2,2-Bis(3-(3-methoxyphenyl)-allyl)-butan-1,3-diol 243}

Polymergebundenes 2,2-Diallylacetoacetat 200 (300 mg, 1 eq.) wurde in trockenem THF (4 ml) gequollen und die Suspension entgast, indem $1 \mathrm{~h}$ lang Argon durch die Reaktionsmischung geleitet wurde. Man fügte 3-Iodanisol (0.52 ml, $0.52 \mathrm{~g}, 2.2 \mathrm{mmol}, 10$ eq.), Triethylamin $(0.091 \mathrm{ml}, 0.066 \mathrm{~g}, 0.66 \mathrm{mmol}, 3$ eq.) und Palladiumacetat $(10 \mathrm{mg}, 0.045 \mathrm{mmol}$, $20 \mathrm{~mol} \%$ ) hinzu und erhitzte $5 \mathrm{~h}$ auf $80^{\circ} \mathrm{C}$. Anschließend wurde abfiltriert, das Harz mit DMF $(20 \mathrm{ml})$, THF $(20 \mathrm{ml}), \mathrm{MeOH}(20 \mathrm{ml})$ und $\mathrm{CH}_{2} \mathrm{Cl}_{2}(20 \mathrm{ml})$ gewaschen und bei $55^{\circ} \mathrm{C}$ im Vakuum getrocknet.

Gemäß AAV 8 wurde die Zielverbindung von der festen Phase abgespalten und man erhielt nach säulenchromatographischer Reinigung an Kieselgel (4 g, Pentan : Essigester = $5: 1$ ) $42 \mathrm{mg}(0.11 \mathrm{mmol}, 53 \%) 243$ als farbloses Öl.

Spektroskopische Daten siehe 4.6.19.

\subsection{3. (E)-2,2-Bis(3-(3-methoxyphenyl)-allyl)-propan-1,3-diol 302}

Polymergebundenes 2,2-Diallyl-methylmalonat 201 (300 mg, 1 eq.) wurde in trockenem $\mathrm{MeCN}$ (4 ml) gequollen und die Suspension entgast, indem $1 \mathrm{~h}$ lang Argon durch die Reakti- 
onsmischung geleitet wurde. Man fügte 3-Iodanisol $(0.52 \mathrm{ml}, 0.52 \mathrm{~g}, 2.2 \mathrm{mmol}, 10 \mathrm{eq}$.), Triethylamin ( $0.091 \mathrm{ml}, 0.066 \mathrm{~g}, 0.66 \mathrm{mmol}, 3$ eq.) und Palladiumacetat $(10 \mathrm{mg}, 0.045 \mathrm{mmol}$, $20 \mathrm{~mol} \%$ ) hinzu und erhitzte für $7 \mathrm{~h}$ auf $80^{\circ} \mathrm{C}$. Anschließend wurde abfiltriert, das Harz mit DMF (20 ml), THF (20 ml), MeOH $(20 \mathrm{ml})$ und $\mathrm{CH}_{2} \mathrm{Cl}_{2}(20 \mathrm{ml})$ gewaschen und bei $55^{\circ} \mathrm{C} \mathrm{im}$ Vakuum getrocknet.

Gemäß AAV 8 wurde die Zielverbindung von der festen Phase abgespalten und man erhielt nach säulenchromatographischer Reinigung an Kieselgel ( $4 \mathrm{~g}$, Pentan : Essigester $=5: 1$ ) $33 \mathrm{mg}(0.090 \mathrm{mmol}, 43 \%) 302$ als farbloses Öl.

$\mathbf{R}_{\mathbf{f}}=0.30$ (Diethylether).

IR (Film): $\widetilde{\mathrm{v}}=3376(\mathrm{OH}), 3012,2968(\mathrm{CH}), 1601(\mathrm{C}=\mathrm{C}), 774,685$ (m-disubst. Aromat) $\mathrm{cm}^{-1}$.

UV $\left(\mathrm{CH}_{3} \mathrm{CN}\right): \lambda_{\max }(\lg \varepsilon)=213.7$ (4.048), 260.8 (3.895), 291.5 (3.998) nm.

${ }^{1}$ H-NMR (200 MHz, $\mathrm{CDCl}_{3}$ ): $\delta=1.76$ (br. s, $\left.2 \mathrm{H}, \mathrm{OH}\right), 2.32$ (d, J = 6.3 Hz, $4 \mathrm{H}, 1$ '- $\mathrm{H}_{2}, 1$ ''$\mathrm{H}_{2}$ ), 3.79 (s, $4 \mathrm{H}, 1-\mathrm{H}_{2}, 3-\mathrm{H}_{2}$ ), 3.82 (s, $\left.6 \mathrm{H}, \mathrm{OMe}\right), 6.28$ (dt, $J=16.9,6.30 \mathrm{~Hz}, 2 \mathrm{H}, 2$ '-H, 2' 'H), 6.46 (d, J=16.9 Hz, 2 H, 3'-H, 3' '-H), 6.74-7.01 (m, 6 H, 5'-H, 7'-H, 9'-H, 5' '-H, 7' '-H, 9' '-H), 7.12-7.25 (m, 2 H, 8'-H, 8' '-H).

${ }^{13}$ C-NMR (50.3 MHz, $\left.\mathrm{CDCl}_{3}\right): \delta=35.36(\mathrm{C}-1$ ', C-1' '), $43.35(\mathrm{C}-2), 55.21(\mathrm{OMe}), 68.14(\mathrm{C}-$ 1, C-3), 111.5 (C-5', C-5'), 112.6 (C-7', C-7'’), 118.7 (C-9', C-9'’), 125.9 (C-2', C-2'’), 129.5 (C-8', C-8' '), 133.1 (C-3', C-3'’), 138.8 (C-4', C-4'”), 159.7 (C-6', C-6'’).

MS (DCI, $\left.\mathrm{NH}_{3}\right): \mathrm{m} / \mathrm{z}=386[\mathrm{M}+18], 403[\mathrm{M}+18+17]$.

$\mathrm{C}_{23} \mathrm{H}_{28} \mathrm{O}_{4}$ (368.46)

\subsection{4. (E)-2,2-Bis(3-phenylallyl)-butan-1,3-diol 239}

Polymergebundenes 2,2-Diallylacetoacetat 200 (300 mg, 1 eq.) wurde in trockenem DMF (4 ml) gequollen und die Suspension entgast, indem $1 \mathrm{~h}$ lang Argon durch die Reaktionsmischung geleitet wurde. Man fügte Brombenzol (0.23 ml, $0.34 \mathrm{~g}, 2.2 \mathrm{mmol}, 10$ eq.), KOAc (65 mg, $0.66 \mathrm{mmol}, 3$ eq.), Tetrabutylammoniumchlorid (61 mg, $0.22 \mathrm{mmol}, 1$ eq.), Palladiumacetat (10 mg, $0.045 \mathrm{mmol}, 20 \mathrm{~mol} \%$ ) und Triphenylphosphan (23 mg, $0.088 \mathrm{mmol}$, $40 \mathrm{~mol} \%$ ) hinzu und erhitzte für $22 \mathrm{~h}$ auf $100{ }^{\circ} \mathrm{C}$. Anschließend wurde abfiltriert, das Harz mit DMF (20 ml), THF (20 ml), MeOH (20 ml) und $\mathrm{CH}_{2} \mathrm{Cl}_{2}(20 \mathrm{ml})$ gewaschen und bei $55^{\circ} \mathrm{C} \mathrm{im}$ Vakuum getrocknet.

Gemäß AAV 8 wurde die Zielverbindung von der festen Phase abgespalten und man erhielt nach säulenchromatographischer Reinigung an Kieselgel (4 g, Pentan : Essigester = $5: 1$ ) $40 \mathrm{mg}(0.12 \mathrm{mmol}, 60 \%) \mathbf{2 3 9}$ als farbloses Öl. 
Spektroskopische Daten siehe 4.6.15.

\subsection{5. (E)-2,2-Bis(3-(3-methoxyphenyl)-allyl)-butan-1,3-diol 243}

Polymergebundenes 2,2-Diallylacetoacetat 200 (300 mg, 1 eq.) wurde in trockenem DMF (4 ml) gequollen und die Suspension entgast, indem $1 \mathrm{~h}$ lang Argon durch die Reaktionsmischung geleitet wurde. Man fügte 3-Bromanisol (0.27 ml, $0.41 \mathrm{~g}, 2.2 \mathrm{mmol}, 10$ eq.), Tributyl$\operatorname{amin}(0.15 \mathrm{ml}, 0.12 \mathrm{~g}, 0.66 \mathrm{mmol}, 3$ eq.), Palladiumacetat $(10 \mathrm{mg}, 0.045 \mathrm{mmol}, 20 \mathrm{~mol} \%)$ und Triphenylphosphan (23 mg, $0.088 \mathrm{mmol}, 40 \mathrm{~mol} \%$ ) hinzu und erhitzte für $24 \mathrm{~h}$ auf $100{ }^{\circ} \mathrm{C}$. Anschließend wurde abfiltriert, das Harz mit DMF (20 ml), THF (20 ml), MeOH (20 ml) und $\mathrm{CH}_{2} \mathrm{Cl}_{2}(20 \mathrm{ml})$ gewaschen und bei $55^{\circ} \mathrm{C}$ im Vakuum getrocknet.

Gemäß AAV 8 wurde die Zielverbindung von der festen Phase abgespalten und man erhielt nach säulenchromatographischer Reinigung an Kieselgel (4 g, Pentan : Essigester $=5: 1$ ) $44 \mathrm{mg}(0.11 \mathrm{mmol}, 55 \%) 243$ als farbloses Öl.

Spektroskopische Daten siehe 4.6.19.

\subsection{6. (E)-2,2-Bis(3-phenylallyl)-propan-1,3-diol 240}

Polymergebundenes 2,2-Diallyl-methylmalonat 201 (300 mg, 1 eq.) wurde in trockenem DMF (4 ml) gequollen und die Suspension entgast, indem $1 \mathrm{~h}$ lang Argon durch die Reaktionsmischung geleitet wurde. Man fügte Brombenzol (0.23 ml, $0.34 \mathrm{~g}, 2.2 \mathrm{mmol}, 10$ eq.), KOAc (65 mg, $0.66 \mathrm{mmol}, 3$ eq.), Tetrabutylammoniumchlorid (61 mg, $0.22 \mathrm{mmol}, 1$ eq.), Palladiumacetat (10 mg, $0.045 \mathrm{mmol}, 20 \mathrm{~mol} \%$ ) und Triphenylphosphan (23 mg, $0.088 \mathrm{mmol}$, $40 \mathrm{~mol} \%$ ) hinzu und erhitzte für $20 \mathrm{~h}$ auf $100{ }^{\circ} \mathrm{C}$. Anschließend wurde abfiltriert, das Harz mit DMF (20 ml), THF (20 ml), MeOH $(20 \mathrm{ml})$ und $\mathrm{CH}_{2} \mathrm{Cl}_{2}(20 \mathrm{ml})$ gewaschen und bei $55^{\circ} \mathrm{C} \mathrm{im}$ Vakuum getrocknet.

Gemäß AAV 8 wurde die Zielverbindung von der festen Phase abgespalten und man erhielt nach säulenchromatographischer Reinigung an Kieselgel (4 g, Pentan : Essigester $=5: 1$ ) $35 \mathrm{mg}(0.11 \mathrm{mmol}, \mathbf{5 5 \% )} \mathbf{2 4 0}$ als farbloses Öl.

Spektroskopische Daten siehe 4.6.16. 


\subsection{Synthesen am Wang-Harz}

\subsubsection{3-Allylhex-5-en-2-on 290}

Gemäß AAV 6 wurde polymergebundenes Acetoacetat 289 (300 mg) mit Essigsäureallylester $19910 \mathrm{~h}$ bei Raumtemperatur gerührt. Dann wurde das Harz $1 \mathrm{~h}$ bei Raumtemperatur in einer 20\%iger Lösung von Trifluoressigsäure in Dichlormethan gerührt, das Harz abfiltriert und mit $30 \mathrm{ml}$ Dichlormethan gewaschen. Nach dem Einengen des Filtrats im Vakuum erhielt man $30 \mathrm{mg}$ (0.72 mmol, 72\%) eines farblosen Öls.

$\mathbf{R}_{\mathbf{f}}=0.75($ Pentan : Essigester $=3: 1)$.

IR (Film): $\widetilde{v}=3034,2972(\mathrm{CH}), 1712(\mathrm{C}=\mathrm{O}), 1642(\mathrm{C}=\mathrm{C}) \mathrm{cm}^{-1}$.

UV $\left(\mathrm{CH}_{3} \mathrm{CN}\right): \lambda_{\max }(\lg \varepsilon)=225.0(2.974) \mathrm{nm}$.

${ }^{1}$ H-NMR $\left(200 \mathrm{MHz}, \mathrm{CDCl}_{3}\right): \delta=2.09$ (s, $\left.3 \mathrm{H}, 1-\mathrm{H}_{3}\right), 2.11-2.38\left(\mathrm{~m}, 4 \mathrm{H}, 4-\mathrm{H}_{2}, 1\right.$ ' $\left.-\mathrm{H}_{2}\right), 2.55$ $\left(\mathrm{m}_{\mathrm{c}}, 1 \mathrm{H}, 3-\mathrm{H}\right) 5.03\left(\mathrm{~d}, J=11.8 \mathrm{~Hz}, 2 \mathrm{H}, 6-\mathrm{H}_{\mathrm{a}}, 3^{\prime}-\mathrm{H}_{\mathrm{a}}\right), 5.07$ (d, J=15.5 Hz, $\left.2 \mathrm{H}, 6-\mathrm{H}_{\mathrm{b}}, 3^{\prime}-\mathrm{H}_{\mathrm{b}}\right)$, 5.81 (ddd, $J=15.5,11.8,7.0 \mathrm{~Hz}, 2 \mathrm{H}, 5-\mathrm{H}, 2$ '-H).

${ }^{13}$ C-NMR (50.3 MHz, CDCl ${ }_{3}$ ): $\delta=29.61$ (C-1), 35.18 (C-4, C-1'), 52.06 (C-3), 117.0 (C-6, C-3'), 135.2 (C-5, C-2'), 210.3 (C-2).

MS (DCI, $\left.\mathrm{NH}_{3}\right): \mathrm{m} / \mathrm{z}=156[\mathrm{M}+18], 173[\mathrm{M}+18+17]$.

$\mathrm{C}_{9} \mathrm{H}_{14} \mathrm{O}$ (138.21)

\subsection{2. (E)-Phenyl-3-(3-phenylallyl)-hex-5-en-2-on 291}

Gemäß AAV 7 wurde polymergebundenes Acetoacetat 289 (300 mg) mit (E)-3-Phenylallylethyl-carbonat 1985 h bei Raumtemperatur gerührt. Dann wurde das Harz 1 h bei Raumtemperatur in einer 20\%iger Lösung von Trifluoressigsäure in Dichlormethan gerührt, das Harz abfiltriert und mit $30 \mathrm{ml}$ Dichlormethan gewaschen. Nach dem Einengen des Filtrats im Vakuum erhielt man $35 \mathrm{mg}$ (0.60 mmol, 60\%) eines farblosen Öls.

$\mathbf{R}_{\mathbf{f}}=0.55($ Pentan : Essigester $=10: 1)$.

IR (Film): $\widetilde{\mathbf{v}}=3358(\mathrm{OH}), 3031,2958(\mathrm{CH}), 1712(\mathrm{C}=\mathrm{O}), 1598(\mathrm{C}=\mathrm{C}), 1448,1378\left(\mathrm{CH}_{3}\right)$ $\mathrm{cm}^{-1}$.

UV $\left(\mathrm{CH}_{3} \mathrm{CN}\right): \lambda_{\max }(\lg \varepsilon)=206.0$ (4.587), 257.0 (4.474), 280.4 (3.014), 291.1 (3.548) nm.

${ }^{1} \mathbf{H}-\mathbf{N M R}\left(200 \mathrm{MHz}, \mathrm{CDCl}_{3}\right): \delta=2.10$ (s, $\left.3 \mathrm{H}, 1-\mathrm{H}_{3}\right), 2.11-2.83\left(\mathrm{~m}, 3 \mathrm{H}, 3-\mathrm{H}, 4-\mathrm{H}_{2}, 1\right.$ '- $\left.\mathrm{H}_{2}\right)$, $6.03(\mathrm{dt}, J=15.1,7.0 \mathrm{~Hz}, 1 \mathrm{H}, 4-\mathrm{H}), 6.13$ (dt, $\left.J=15.1,7.0 \mathrm{~Hz}, 1 \mathrm{H}, 2^{\prime}-\mathrm{H}\right), 6.41$ (d, $J=15.1$ $\mathrm{Hz}, 1 \mathrm{H}, 6-\mathrm{H}), 6.48$ (d, J = 15.1 Hz, 1 H, 3’-H), 7.11-7.43 (m, $10 \mathrm{H}, \mathrm{Ph}-\mathrm{H})$. 
${ }^{13}$ C-NMR (50.3 MHz, $\mathrm{CDCl}_{3}$ ): $\delta=29.90$ (C-1), 34.47 (C-4, C-2'), 52.69 (C-3), 126.0 (C-8, C-12, C-5', C-9'), 126.2 (C-10, C-7'), 127.2 (C-4, C-2'), 128.4 (C-9, C-11, C-6', C-8'), 132.3 (C-6, C-3'), 137.1 (C-7, C-5'), 210.1 (C-2).

MS $\left(\mathrm{DCI}, \mathrm{NH}_{3}\right): \mathrm{m} / \mathrm{z}=291[\mathrm{M}+1], 308$ [M+18], 325 [M+18+17].

$\mathrm{C}_{21} \mathrm{H}_{22} \mathrm{O}$ (290.40)

\subsection{3. (E)-2,7,12-Triacetyl-tridec-4,9-diensäure-dimethylester 295}

Gemäß AAV 7 wurde polymergebundenes Acetoacetat 289 (300 mg) mit (Z)-Ethyl-4hydroxybut-2-encarbonat 275 (5 eq.) $15 \mathrm{~h}$ bei Raumtemperatur gerührt und in Anlehnung an AAV 3 das erhaltene Produkt mit Essigsäureanhydrid (10 eq.) acetyliert. In Anlehnung an AAV 6 wurde das polymergebundene Allylacetat 292 mit Methylacetoacetat 205 (20 eq.) $5 \mathrm{~h}$ am Rückfluß erhitzt. Dann wurde das Harz 1 h bei Raumtemperatur in einer 20\%iger Lösung von Trifluoressigsäure in Dichlormethan gerührt, das Harz abfiltriert und mit $30 \mathrm{ml}$ Dichlormethan gewaschen. Nach dem Einengen des Filtrats im Vakuum erhielt man $53 \mathrm{mg}$ (0.66 mmol, 66\%) eines farblosen Öls.

$\mathbf{R}_{\mathbf{f}}=0.15$ (Pentan : Essigester $\left.=3: 1\right)$.

IR (Film): $\widetilde{v}=2980,2963$ (CH), 1736 (Ester), 1709 (Keton), 1648 (C=C) $\mathrm{cm}^{-1}$.

$\mathbf{U V}\left(\mathrm{CH}_{3} \mathrm{CN}\right): \lambda_{\max }(\lg \varepsilon)=236.0(4.015) \mathrm{nm}$.

${ }^{1}$ H-NMR $\left(200 \mathrm{MHz}, \mathrm{CDCl}_{3}\right): \delta=2.05-2.39\left(\mathrm{~m}, 5 \mathrm{H}, 6-\mathrm{H}_{2}, 7-\mathrm{H}, 8-\mathrm{H}_{2}\right), 2.20$ (s, $3 \mathrm{H}, 7-$ $\left.\mathrm{COCH}_{3}\right), 2.22\left(\mathrm{~s}, 6 \mathrm{H}, 2-\mathrm{COCH}_{3}, 12-\mathrm{COCH}_{3}\right), 2.55\left(\mathrm{~m}_{\mathrm{c}}, 4 \mathrm{H}, 3-\mathrm{H}_{2}, 11-\mathrm{H}_{2}\right), 3.50(\mathrm{t}, J=7.3$ $\mathrm{Hz}, 2 \mathrm{H}, 2-\mathrm{H}, 12-\mathrm{H}), 3.65$ (s, $\left.6 \mathrm{H}, 1-\mathrm{OCH}_{3}, 13-\mathrm{OCH}_{3}\right)$, 5.20-5.51 (m, 4 H, 4-H, 5-H, 9-H, $10-$ $\mathrm{H})$.

${ }^{13}$ C-NMR $\left(50.3 \mathrm{MHz}, \mathrm{CDCl}_{3}\right): \delta=29.17\left(\mathrm{COCH}_{3}\right), 31.07(\mathrm{C}-3, \mathrm{C}-11), 33.68(\mathrm{C}-6, \mathrm{C}-8)$, 52.39 (C-2), 52.39 (C-2), $52.44\left(\mathrm{OCH}_{3}\right), 128.2,128.3$ (C-5, C-9), 129.9, 129.9 (C-4, C-10), 169.6 (C-1, C-13), 202.4 (2-C=O, 12-C=O), $211.3(7-\mathrm{C}=\mathrm{O})$.

MS $\left(\mathrm{DCI}, \mathrm{NH}_{3}\right): \mathrm{m} / \mathrm{z}=416[\mathrm{M}+18]$.

$\mathrm{C}_{21} \mathrm{H}_{34} \mathrm{O}_{7}$ (398.49) 


\section{Festphasensynthese von Pyrazolonen}

\subsubsection{3-Methyl-1-(4-trifluoromethyl-phenyl)-pyrazol-5-on 316}

Gemäß AAV 10 wurde polymergebundenes Acetoacetat 138 (300 mg, 1 eq.) mit 4Trifluoromethyl-phenylhydrazin 311 (10 eq.) umgesetzt. Man erhielt $52 \mathrm{mg}$ (0.21 mmol, 96\%) der Zielverbindung als gelbliche Kristalle.

$\mathbf{R}_{\mathbf{f}}=0.65\left(\mathrm{Et}_{2} \mathrm{O}\right)$.

IR (Pille): $\widetilde{\mathrm{v}}=$ 3025, $2922(\mathrm{CH})$, 1737, $1719(\mathrm{C}=\mathrm{O}), 1602(\mathrm{C}=\mathrm{C}), 1165\left(\mathrm{CF}_{3}\right), 820$ (p-disubst. Aromat) $\mathrm{cm}^{-1}$.

UV $\left(\mathrm{CH}_{3} \mathrm{CN}\right): \lambda_{\max }(\lg \varepsilon)=199.5$ (3.874), 228.1 (3.277), 367.5 (3.572) nm.

${ }^{1} \mathbf{H}$-NMR $\left(200 \mathrm{MHz}, \mathrm{CDCl}_{3}\right): \delta=2.23\left(\mathrm{~s}, 3 \mathrm{H}, 3-\mathrm{CH}_{3}\right), 3.48\left(\mathrm{~s}, 2 \mathrm{H}, 4-\mathrm{H}_{2}\right), 7.64$ (d, $J=9.0$ Hz, 2 H, 3'-H, 5'-H), 8.05 (d, $J=9.0$ Hz, 2 H, 2'-H, 6'-H).

${ }^{13}$ C-NMR (50.3 MHz, $\left.\mathrm{CDCl}_{3}\right): \delta=17.02\left(3-\mathrm{CH}_{3}\right), 43.10$ (C-4), 118.03 (C-2', C-6'), 120.1 ( $\left.\mathrm{CF}_{3}\right), 125.9$ (C-3', C-5'), 126.5 (C-4'), 141.3 (C-1'), 156.9 (C-3), 170.7 (C-5).

MS $(70 \mathrm{eV}): \mathrm{m} / \mathrm{z}(\%)=242(100)\left[\mathrm{M}^{+}\right], 223(15)\left[\mathrm{M}^{+}-\mathrm{F}\right], 173(20)\left[\mathrm{M}^{+}-\mathrm{CF}_{3}\right], 145(70)\left[\mathrm{M}^{+}-\right.$ $\mathrm{C}_{6} \mathrm{H}_{4} \mathrm{CF}_{3}$ ].

$\mathbf{C}_{11} \mathbf{H}_{9} \mathbf{F}_{3} \mathbf{N}_{2} \mathbf{O}(\mathbf{2 4 2 . 1 9 )} \quad$ Ber. 242.0667

Gef. 242.0667 (HRMS)

\subsubsection{3-Methyl-1-(4-methansulfonyl-phenyl)-pyrazol-5-on 317}

Gemäß AAV 10 wurde polymergebundenes Acetoacetat 138 (300 mg, 1 eq.) mit 4Methansulfonyl-phenylhydrazin 312 (10 eq.) umgesetzt. Es wurden 54 mg (0.21 mmol, 94\%) der Titelverbindung als gelbliche Kristalle erhalten.

$\mathbf{R}_{\mathbf{f}}=0.35\left(\mathrm{Et}_{2} \mathrm{O}\right)$.

IR (Pille): $\widetilde{\mathrm{v}}=2920(\mathrm{CH}), 1716(\mathrm{C}=\mathrm{O}), 1598(\mathrm{C}=\mathrm{C}), 1451,1360\left(\mathrm{CH}_{3}\right), 820$ (p-disubst. Aromat) $\mathrm{cm}^{-1}$.

UV $\left(\mathrm{CH}_{3} \mathrm{CN}\right): \lambda_{\max }(\lg \varepsilon)=201.5(3.415), 221.5$ (3.457), $358.5(3.447) \mathrm{nm}$.

${ }^{1}$ H-NMR $\left(200 \mathrm{MHz}, \mathrm{CDCl}_{3}\right): \delta=2.23\left(\mathrm{~s}, 3 \mathrm{H}, 3-\mathrm{CH}_{3}\right), 3.06\left(\mathrm{~s}, 3 \mathrm{H}, \mathrm{SO}_{2} \mathrm{CH}_{3}\right), 3.50(\mathrm{~s}, 2 \mathrm{H}$, 4-H ${ }_{2}$ ), 7.93 (d, $J=9.4$ Hz, 2 H, 3'-H, 5'-H), 8.15 (d, $J=9.4$ Hz, 2 H, 2'-H, 6'-H). 
${ }^{13}$ C-NMR (50.3 MHz, $\left.\mathrm{CDCl}_{3}\right): \delta=17.02\left(3-\mathrm{CH}_{3}\right), 43.08(\mathrm{C}-4), 44.59\left(\mathrm{SO}_{2} \mathrm{CH}_{3}\right), 118.1(\mathrm{C}-2$ ', C-6'), 128.3 (C-3', C-5'), 135.4 (C-4'), 142.2 (C-1'), 157.5 (C-3), 170.9 (C-5).

MS $(70 \mathrm{eV}): \mathrm{m} / \mathrm{z}(\%)=252(100)\left[\mathrm{M}^{+}\right], 237(15)\left[\mathrm{M}^{+}-\mathrm{CH}_{3}\right], 173(15)\left[\mathrm{M}^{+}-\mathrm{SO}_{2} \mathrm{CH}_{3}\right], 155(20)$ $\left[\mathrm{M}^{+}-\mathrm{C}_{6} \mathrm{H}_{4} \mathrm{SO}_{2} \mathrm{CH}_{3}\right]$.

$\mathbf{C}_{11} \mathbf{H}_{12} \mathbf{N}_{2} \mathbf{O}_{3} \mathbf{S}(\mathbf{2 5 2 . 2 9 )} \quad$ Ber. 252.0569

Gef. 252.0569 (HRMS)

\subsubsection{3-Methyl-1-(4-nitrophenyl)-pyrazol-5-on 318}

Gemäß AAV 10 wurde polymergebundenes Acetoacetat 138 (300 mg, 1 eq.) mit 4Nitrophenylhydrazin 313 (10 eq.) umgesetzt. Man erhielt $24 \mathrm{mg}$ (1.09 mmol, 52\%) der gewünschten Verbindung als leicht gelbe Kristalle.

$\mathbf{R}_{\mathbf{f}}=0.45\left(\mathrm{Et}_{2} \mathrm{O}\right)$.

IR (Pille): $\widetilde{v}=3033,2960(\mathrm{CH}), 1625(\mathrm{C}=\mathrm{O}), 1597,1580(\mathrm{C}=\mathrm{C}), 1429,1367(\mathrm{CH}), 1505$, $1367\left(\mathrm{NO}_{2}\right), 822$ (p-disubst. Aromat) $\mathrm{cm}^{-1}$.

UV $\left(\mathrm{CH}_{3} \mathrm{CN}\right): \lambda_{\max }(\lg \varepsilon)=196.5$ (3.563), 223.0 (3.237), $342.5(3.282) \mathrm{nm}$.

${ }^{1} \mathbf{H}$-NMR $\left(200 \mathrm{MHz}, \mathrm{CDCl}_{3}\right): \delta=2.25\left(\mathrm{~s}, 3 \mathrm{H}, 3-\mathrm{CH}_{3}\right), 3.50\left(\mathrm{~s}, 2 \mathrm{H}, 4-\mathrm{H}_{2}\right), 8.12(\mathrm{~d}, J=9.4$ Hz, 2 H, 2'-H, 6'-H), 8.26 (d, $J=9.4$ Hz, 2 H, 3'-H, 5'-H).

${ }^{13}$ C-NMR $\left(50.3 \mathrm{MHz}, \mathrm{CDCl}_{3}\right): \delta=17.12\left(3-\mathrm{CH}_{3}\right), 41.69$ (C-4), 121.4 (C-2', C-6'), $123.5(\mathrm{C}-$ 3', C-5'), 144.3 (C-4'), 146.9 (C-1'), 157.4 (C-3), 170.9 (C-5).

MS $(70 \mathrm{eV}): \mathrm{m} / \mathrm{z}(\%)=219(100)\left[\mathrm{M}^{+}\right], 173(5)\left[\mathrm{M}^{+}-\mathrm{NO}_{2}\right], 122(30)\left[\mathrm{C}_{6} \mathrm{H}_{4} \mathrm{NO}_{2}{ }^{+}\right]$.

$\mathbf{C}_{10} \mathbf{H}_{9} \mathbf{N}_{3} \mathbf{O}_{3}$ (219.19) Ber. 219.0644

Gef. 219.0643 (HRMS)

\subsubsection{1-(2-Chloro-6,7-dihydro-5H-cyclopentapyrimidin-4-yl)-3-methyl-pyrazol- 5-on 319}

Gemäß AAV 10 wurde polymergebundenes Acetoacetat 138 (300 mg, 1 eq.) mit 2-Chloro6,7-dihydro-5H-cyclopentapyrimidin-4-yl-hydrazin 314 (10 eq.) umgesetzt. Es wurden $31 \mathrm{mg}$ (0.12 mmol, 54\%) der Titelverbindung als gelbliche-rote Kristalle erhalten.

$\mathbf{R}_{\mathbf{f}}=0.30\left(\mathrm{Et}_{2} \mathrm{O}\right)$.

IR (Pille): $\widetilde{\mathrm{v}}=2972,2926(\mathrm{CH}), 1635(\mathrm{C}=\mathrm{O}) \mathrm{cm}^{-1}$.

UV $\left(\mathrm{CH}_{3} \mathrm{CN}\right): \lambda_{\max }(\lg \varepsilon)=197.5$ (3.808), 225.0 (3.783), 243.5 (3.759), 290.5 (3.952) nm. 
${ }^{1}$ H-NMR (200 MHz, $\mathrm{CDCl}_{3}$ ), OH-Form: $\delta=2.22$ (br. s, $\left.4 \mathrm{H}, 3-\mathrm{CH}_{3}, \mathrm{OH}\right), 2.72-3.03$ (m, $2 \mathrm{H}$, 6'- $-\mathrm{H}_{2}$ ), 3.04 (t, $\left.J=7.8 \mathrm{~Hz}, 2 \mathrm{H}, 7^{\prime}-\mathrm{H}_{2}\right), 3.41$ (d, $\left.J=7.8 \mathrm{~Hz}, 2 \mathrm{H}, 5^{\prime}-\mathrm{H}_{2}\right), 5.45$ (s, $\left.1 \mathrm{H}, 4-\mathrm{H}\right)$.

${ }^{13}$ C-NMR (75.5 MHz, CD 3 OD): $\delta=13.49$ (3- $\left.\mathrm{CH}_{3}\right), 23.19$ (C-7'), 31.72 (C-6'), 35.38 (C-5'), 43.31 (C-4), 124.0 (C-7 '), 154.8 (C-3’), 154.9 (C-3), 164.5 (C-1'), 170.9 (C-5), 182.9 (C$\left.4{ }^{\prime}{ }^{\prime}\right)$.

MS (DCI): $\mathrm{m} / \mathrm{z}(\%)=251\left[\mathrm{M}^{+}+1\right], 268\left[\mathrm{M}^{+}+18\right]$.

$\mathrm{C}_{11} \mathrm{H}_{11} \mathrm{ClN}_{4} \mathrm{O}_{2}$ (250.68)

Ber. 250.0621

Gef. 250.0621 (HRMS)

\subsubsection{3-Methyl-1-(2,2,2-trifluoroethyl)-pyrazol-5-on 320}

Gemäß AAV 10 wurde polymergebundenes Acetoacetat 138 (300 mg, 1 eq.) mit 2,2,2Trifluoroethylhydrazin 315 (10 eq.) umgesetzt. Man erhielt $19 \mathrm{mg}$ (1.05 mmol, 94\%) der gewünschten Verbindung als leicht gelbe Kristalle.

$\mathbf{R}_{\mathbf{f}}=0.50\left(\mathrm{Et}_{2} \mathrm{O}\right)$.

IR (Pille): $\widetilde{\mathrm{v}}=$ 3010, $2933(\mathrm{CH}), 1635(\mathrm{C}=\mathrm{O}), 1600(\mathrm{C}=\mathrm{C}), 1458,1403\left(\mathrm{CH}_{3}\right), 1154\left(\mathrm{CF}_{3}\right)$ $\mathrm{cm}^{-1}$.

UV $\left(\mathrm{CH}_{3} \mathrm{CN}\right): \lambda_{\max }(\lg \varepsilon)=241.5(3.049) \mathrm{nm}$.

${ }^{1}$ H-NMR $\left(200 \mathrm{MHz}, \mathrm{CDCl}_{3}\right): \delta=2.14\left(\mathrm{~s}, 3 \mathrm{H}, 3-\mathrm{CH}_{3}\right), 3.26$ (s, $\left.2 \mathrm{H}, 4-\mathrm{H}_{2}\right), 4.23$ (q, $J=8.9$ $\left.\mathrm{Hz}, 2 \mathrm{H}, 1^{\prime}-\mathrm{H}_{2}\right)$.

${ }^{13}$ C-NMR (50.3 MHz, $\left.\mathrm{CDCl}_{3}\right): \delta=17.01\left(3-\mathrm{CH}_{3}\right), 41.52(\mathrm{C}-4), 58.57$ (q, $J=30.0 \mathrm{~Hz}, \mathrm{C}-1$ ') 110.4 (q, $J=278.8 \mathrm{~Hz}, \mathrm{C}-2$ ') 157.4 (C-3), 170.1 (C-5).

MS $(70 \mathrm{eV}): \mathrm{m} / \mathrm{z}(\%)=180(100)\left[\mathrm{M}^{+}\right], 161(15)\left[\mathrm{M}^{+}-\mathrm{F}\right], 111(60)\left[\mathrm{M}^{+}-\mathrm{CF}_{3}\right], 69(45)\left[\mathrm{CF}_{3}^{+}\right]$.

$\mathbf{C}_{6} \mathbf{H}_{7} \mathbf{F}_{3} \mathbf{N}_{2} \mathbf{O}$ (180.12) Ber. 180.0510

Gef. 180.0510 (HRMS)

\subsubsection{3-Methyl-1-(2-nitrophenyl)-pyrazol-5-on 323}

In Anlehnung an AAV 10 wurde polymergebundenes Acetoacetat 138 (300 mg, 1 eq.) mit 2Nitrophenylhydrazin 321 (10 eq.) umgesetzt. Die Abspaltung der Titelverbindung vom polymeren Träger erfolgte durch Erwärmen der Harzes in einer 1\%igen Lösung von TFA in Toluol auf $80{ }^{\circ} \mathrm{C}$ für $5 \mathrm{~h}$. Man erhielt $26 \mathrm{mg}(1.19 \mathrm{mmol}, 57 \%)$ der gewünschten Verbindung als leicht rötliche Kristalle. 
$\mathbf{R}_{\mathbf{f}}=0.40\left(\mathrm{Et}_{2} \mathrm{O}\right)$.

IR (Pille): $\widetilde{v}=$ 3033, $2960(\mathrm{CH}), 1625(\mathrm{C}=\mathrm{O}), 1597,1580(\mathrm{C}=\mathrm{C}), 1429,1367(\mathrm{CH}), 1505$, $1367\left(\mathrm{NO}_{2}\right) \mathrm{cm}^{-1}$.

UV $\left(\mathrm{CH}_{3} \mathrm{CN}\right): \lambda_{\max }(\lg \varepsilon)=198.2(3.015), 253.60(3.685), 348.5(3.287) \mathrm{nm}$.

${ }^{1}$ H-NMR $\left(200 \mathrm{MHz}, \mathrm{CDCl}_{3}\right): \delta=2.20\left(\mathrm{~s}, 3 \mathrm{H}, 3-\mathrm{CH}_{3}\right), 3.42\left(\mathrm{~s}, 2 \mathrm{H}, 4-\mathrm{H}_{2}\right), 7.39-7.48(\mathrm{~m}, 1$ H, 4'-H), 7.64-7.69 (m, 2 H, 5'-H, 6'-H), 7.94 (d, J = 7.8 Hz, 1 H, 3'-H).

${ }^{13} \mathrm{C}-\mathrm{NMR}\left(50.3 \mathrm{MHz}, \mathrm{CDCl}_{3}\right): \delta=17.07\left(3-\mathrm{CH}_{3}\right), 41.74$ (C-4), 125.1 (C-6'), 126.0 (C-3'), 127.4 (C-4'), 129.7 (C-1'), 133.3 (C-5'), 143.4 (C-2'), 157.6 (C-3), 170.8 (C-5).

MS (DCI, $\left.\mathrm{NH}_{3}\right): \mathrm{m} / \mathrm{z}=237[\mathrm{M}+18]$.

$\mathbf{C}_{\mathbf{1 0}} \mathbf{H}_{9} \mathbf{N}_{3} \mathbf{O}_{3}$ (219.19) $\quad$ Ber. 219.0643

Gef. 219.0643 (HRMS)

\subsubsection{1-(2,4-Difluorophenyl)-3-methyl-pyrazol-5-on 327}

2,4-Difluorophenylhydrazin 324 wurde durch Waschen mit ges. $\mathrm{NaHCO}_{3}$-Lösung $(5 \mathrm{ml})$ aus dem entsprechenden Hydrochlorid freigesetzt. Die wäßrige Phase extrahierte man mit Essigester (dreimal $3 \mathrm{ml}$ ), wusch die vereinigte organische Phase mit ges. NaCl-Lösung, trocknete über $\mathrm{Na}_{2} \mathrm{SO}_{4}$ und engte im Vakuum ein.

Gemäß AAV 10 wurde polymergebundenes Acetoacetat 138 (300 mg, 1 eq.) mit 2,4Difluorophenylhydrazin 324 (10 eq.) umgesetzt. Es wurden $22 \mathrm{mg}$ (1.05 mmol, 50\%) der gewünschten Verbindung als leicht gelbe Kristalle erhalten.

$\mathbf{R}_{\mathbf{f}}=0.45\left(\mathrm{Et}_{2} \mathrm{O}\right)$.

IR (Pille): $\widetilde{\text { v }}=$ 3082, $2962(\mathrm{CH}), 1731(\mathrm{C}=\mathrm{O}), 1673(\mathrm{C}=\mathrm{C}), 1437,1370\left(\mathrm{CH}_{3}\right), 851$ (unsym. trisubst. Aromat) $\mathrm{cm}^{-1}$.

UV $\left(\mathrm{CH}_{3} \mathrm{CN}\right): \lambda_{\max }(\lg \varepsilon)=233.5$ (3.914), 260.0 (3.609) $\mathrm{nm}$.

${ }^{1} \mathbf{H}-\mathbf{N M R}\left(200 \mathrm{MHz}, \mathrm{CDCl}_{3}\right): \delta=2.19\left(\mathrm{~s}, 3 \mathrm{H}, 3-\mathrm{CH}_{3}\right), 3.41$ (s, $\left.2 \mathrm{H}, 4-\mathrm{H}_{2}\right), 6.90-7.02$ (m, 2 H, 3'-H 5'-H), 7.34-7.49 (m, 1 H, 6'-H).

${ }^{13}$ C-NMR (50.3 MHz, $\left.\mathrm{CDCl}_{3}\right): \delta=17.09\left(3-\mathrm{CH}_{3}\right), 41.40(\mathrm{C}-4), 105.1(\mathrm{dd}, J=26.3,23.6 \mathrm{~Hz}$, C-3'), 111.6 (dd, $J=22.3,3.4 \mathrm{~Hz}, \mathrm{C}-5$ ') 121.0 (C'-1), 128.2 (dd, $J=9.5,2.0 \mathrm{~Hz}, \mathrm{C}-6$ ') 156 (dd, $\left.J=43.1,7.1 \mathrm{~Hz}, \mathrm{C}-2^{\prime}\right), 164.3$ (C-4').

MS (70 eV): m/z (\%) = $210(100)\left[\mathrm{M}^{+}\right], 127(65)\left[\mathrm{C}_{6} \mathrm{H}_{3} \mathrm{~F}_{2} \mathrm{~N}^{+}\right], 113(35)\left[\mathrm{C}_{6} \mathrm{H}_{3} \mathrm{~F}_{2}^{+}\right]$.

$\mathrm{C}_{10} \mathrm{H}_{8} \mathrm{~F}_{2} \mathrm{~N}_{2} \mathrm{O}(210.18)$

Ber. 194.1419

Gef. 194.1419 (HRMS) 


\subsubsection{3-Methyl-1-(1-phenylethyl)-pyrazol-5-on 328}

1-Phenylethylhydrazin 325 wurde durch Waschen mit ges. $\mathrm{NaHCO}_{3}$-Lösung (5 ml) aus dem entsprechenden Hydrochlorid freigesetzt. Die wäßrige Phase extrahierte man mit Essigester (dreimal $3 \mathrm{ml}$ ), wusch die vereinigte organische Phase mit ges. NaCl-Lösung, trocknete über $\mathrm{Na}_{2} \mathrm{SO}_{4}$ und engte im Vakuum ein.

Gemäß AAV 10 wurde polymergebundenes Acetoacetat 138 (300 mg, 1 eq.) mit 1Phenylethylhydrazin 325 (10 eq.) umgesetzt. Es wurden $26 \mathrm{mg}$ (1.32 mmol, 63\%) der Titelverbindung als rötliches Öl erhalten. 328 liegt nach ${ }^{1} \mathrm{H}-\mathrm{NMR}$-spektroskopischer Untersuchung als 2.5 : 1 -Gemisch aus OH- und Keto-Tautomer vor.

$\mathbf{R}_{\mathbf{f}}=0.25\left(\mathrm{Et}_{2} \mathrm{O}\right)$.

IR (Film): $\widetilde{v}=3034,2985(\mathrm{CH}), 1672(\mathrm{C}=\mathrm{O}), 1453,1370\left(\mathrm{CH}_{3}\right) \mathrm{cm}^{-1}$.

UV $\left(\mathrm{CH}_{3} \mathrm{CN}\right): \lambda_{\max }(\lg \varepsilon)=252.5(3.422) \mathrm{nm}$.

${ }^{1}$ H-NMR (200 MHz, $\mathrm{CDCl}_{3}$ ), OH-Tautomer: $\delta=1.83\left(\mathrm{~d}, J=8.1 \mathrm{~Hz}, 3 \mathrm{H}, 1\right.$ '- $\left.\mathrm{CH}_{3}\right), 2.12$ (s, 3 H, 3- $\mathrm{CH}_{3}$ ), 5.46 (s, $\left.1 \mathrm{H}, 4-\mathrm{H}\right), 5.65$ (q, $J=8.1 \mathrm{~Hz}, 1 \mathrm{H}, 1$ '-H), 7.19-7.43 (m, $\left.5 \mathrm{H}, \mathrm{Ph}-\mathrm{H}\right)$.

${ }^{1} \mathbf{H}-\mathbf{N M R}\left(200 \mathrm{MHz}, \mathrm{CDCl}_{3}\right)$, Keto-Tautomer: $\delta=1.69$ (d, $J=8.0 \mathrm{~Hz}, 3 \mathrm{H}, 1$ ' - $\mathrm{CH}_{3}$ ), 2.08 (s, $3 \mathrm{H}, 3-\mathrm{CH}_{3}$ ), 3.18 (s, $2 \mathrm{H}, 4-\mathrm{H}_{2}$ ), 5.42 (q, J = 8.0 Hz, $\left.1 \mathrm{H}, 1^{\prime}-\mathrm{H}\right), 7.19-7.43$ (m, $5 \mathrm{H}, \mathrm{Ph}-\mathrm{H}$ ).

${ }^{13}$ C-NMR (50.3 MHz, $\left.\mathrm{CDCl}_{3}\right)$, OH-Tautomer: $\delta=11.61\left(3-\mathrm{CH}_{3}\right), 18.00\left(1\right.$ ' $\left.-\mathrm{CH}_{3}\right), 55.19(\mathrm{C}-$ 1'), 91.06 (C-4), 121.0 (C-1), 126.6 (C-3', C-7'), 128.3 (C-5'), 128.75 (C-4', C-6'), 138.3 (C5), 146.6 (C-2'), 158.3 (C-3).

MS $(70 \mathrm{eV}): \mathrm{m} / \mathrm{z}(\%)=202(30)\left[\mathrm{M}^{+}\right], 187(45)\left[\mathrm{M}^{+}-\mathrm{CH}_{3}\right], 105(100)\left[\mathrm{M}^{+}-\mathrm{C}_{6} \mathrm{H}_{5} \mathrm{CH}_{2} \mathrm{CH}_{3}\right]$, $77(35)\left[\mathrm{C}_{6} \mathrm{H}_{5}^{+}\right]$.

$\mathrm{C}_{12} \mathrm{H}_{14} \mathrm{~N}_{2} \mathrm{O}(\mathbf{2 0 2 . 2 5 )}$

Ber. 202.1106

Gef. 202.1106 (HRMS)

\subsubsection{3-Methyl-1-(2-methyl-cyclohexyl)-pyrazol-5-on 329}

2-Methyl-cyclohexylhydrazin 326 wurde durch Waschen mit ges. $\mathrm{NaHCO}_{3}$-Lösung $(5 \mathrm{ml})$ aus dem entsprechenden Hydrochlorid freigesetzt. Die wäßrige Phase extrahierte man mit Essigester (dreimal $3 \mathrm{ml}$ ), wusch die vereinigte organische Phase mit ges. NaCl-Lösung, trocknete über $\mathrm{Na}_{2} \mathrm{SO}_{4}$ und engte im Vakuum ein.

Gemäß AAV 10 wurde polymergebundenes Acetoacetat 138 (300 mg, 1 eq.) mit 2-Methylcyclohexylhydrazin 326 (10 eq.) umgesetzt. Es wurden $18 \mathrm{mg}$ (0.94 mmol, 45\%) der Titelverbindung als gelbliches Öl erhalten. 329 liegt nach ${ }^{1} \mathrm{H}-\mathrm{NMR}$-spektroskopischer Untersuchung als $2: 1$-Gemisch aus OH- und Keto-Tautomer vor. 
$\mathbf{R}_{\mathbf{f}}=0.25\left(\mathrm{Et}_{2} \mathrm{O}\right)$.

IR (Film): $\widetilde{v}=2935(\mathrm{CH}), 1674(\mathrm{C}=\mathrm{O}), 1448,1385\left(\mathrm{CH}_{3}\right) \mathrm{cm}^{-1}$.

UV $\left(\mathrm{CH}_{3} \mathrm{CN}\right): \lambda_{\max }(\lg \varepsilon)=254.5(3.367) \mathrm{nm}$.

${ }^{1}$ H-NMR (200 MHz, $\mathrm{CDCl}_{3}$ ), OH-Tautomer: $\delta=0.85$ (d, $J=7.1 \mathrm{~Hz}, 3 \mathrm{H}, 2$ '- $\mathrm{CH}_{3}$ ), 1.28-2.05 (m, $\left.8 \mathrm{H}, 3^{\prime}-\mathrm{H}_{2}, 4^{\prime}-\mathrm{H}_{2}, 5^{\prime}-\mathrm{H}_{2}, 6^{\prime}-\mathrm{H}_{2}\right), 2.23$ (s, $\left.3 \mathrm{H}, 3-\mathrm{CH}_{3}\right), 2.28-2.40$ (m, 1 H, 2'-H), 4.42 (dt, $J=13.2,4.3 \mathrm{~Hz}, 1 \mathrm{H}, 1$ '-H), 5.60 (s, $1 \mathrm{H}, 4-\mathrm{H})$.

${ }^{1} \mathbf{H}$-NMR $\left(200 \mathrm{MHz}, \mathrm{CDCl}_{3}\right)$, Keto-Tautomer: $\delta=0.91\left(\mathrm{~d}, J=7.6 \mathrm{~Hz}, 3 \mathrm{H}, 2^{\prime}-\mathrm{CH}_{3}\right), 1.28$ 2.05 (m, 8 H, 3'- $\left.\mathrm{H}_{2}, 4^{\prime}-\mathrm{H}_{2}, 5^{\prime}-\mathrm{H}_{2}, 6^{\prime}-\mathrm{H}_{2}\right), 2.23$ (s, 3 H, 3- $\mathrm{CH}_{3}$ ), 2.28-2.40 (m, 1 H, 2'-H), 3.21 (s, $\left.2 \mathrm{H}, 4-\mathrm{H}_{2}\right), 4.18$ (dt, $\left.J=8.4,4.3 \mathrm{~Hz}, 1 \mathrm{H}, 1^{\prime}-\mathrm{H}\right)$.

${ }^{13}$ C-NMR $\left(50.3 \mathrm{MHz}, \mathrm{CDCl}_{3}\right)$, OH-Tautomer: $\delta=11.56\left(3-\mathrm{CH}_{3}\right), 12.13\left(2\right.$ ' $\left.-\mathrm{CH}_{3}\right), 19.14(\mathrm{C}-$ 5'), 23.47 (C-4'), 25.80 (C-6'), 31.39 (C-3'), 32.37 (C-2'), 60.06 (C-1'), 91.08 (C-4), 145.5 (C-5), $157.6(\mathrm{C}-3)$.

${ }^{13}$ C-NMR (50.3 MHz, $\left.\mathrm{CDCl}_{3}\right)$, Keto-Tautomer: $\delta=14.35\left(2^{\prime}-\mathrm{CH}_{3}\right), 17.30\left(3-\mathrm{CH}_{3}\right), 19.08(\mathrm{C}-$ 5'), 21.42 (C-4'), 26.56 (C-6'), 31.16 (C-3'), 33.20 (C-2'), 41.75 (C-4), 54.65 (C-1'), 154.1 (C-3), $171.03(\mathrm{C}-5)$.

MS (DCI): $\mathrm{m} / \mathrm{z}(\%)=195\left[\mathrm{M}^{+}+1\right], 212\left[\mathrm{M}^{+}+18\right]$.

$\mathrm{C}_{11} \mathrm{H}_{18} \mathrm{~N}_{2} \mathrm{O}(194.27)$

Ber. 194.1419

Gef. 194.1419 (HRMS) 


\section{Verzeichnis der verwendeten Abkürzungen}

\begin{tabular}{|c|c|}
\hline AAV & Allgemeine Arbeitsvorschrift \\
\hline Ac & Acetyl \\
\hline ber. & berechnet \\
\hline BSA & $\mathrm{N}, \mathrm{O}$-Bis(trimethylsilyl)acetamid \\
\hline $\mathrm{dba}$ & Dibenzylidenaceton \\
\hline DC & Dünnschichtchromatographie \\
\hline $\mathrm{DCC}$ & Dicyclohexylcarbodiimid \\
\hline DIBAH & Diisobutylaluminiumhydrid \\
\hline DMF & Dimethylformamid \\
\hline DMAP & 4-(Dimethylamino)pyridin \\
\hline dppe & 1,2-(Bis(diphenylphosphino)-ethan \\
\hline ee & Enantiomerenüberschuß \\
\hline gef. & gefunden \\
\hline ges. & gesättigt(e) \\
\hline $\mathrm{h}$ & Stunde \\
\hline Kat. & Katalysator \\
\hline konz. & konzentriert \\
\hline LDA & Lithiumdiisopropylamid \\
\hline M & Molar \\
\hline MS & Massenspektroskopie \\
\hline $\mathrm{Nu}$ & Nucleophil \\
\hline org. & $\operatorname{organisch}(e)$ \\
\hline Py & Pyridin \\
\hline $\mathrm{R}_{\mathrm{f}}$ & Retentionsfaktor \\
\hline RT & Raumtemperatur \\
\hline TBAF & Tetrabutylammoniumfluorid \\
\hline TFA & Trifluoressigsäure \\
\hline $\mathrm{THF}$ & Tetrahydrofuran \\
\hline TMS & Trimethylsilyl \\
\hline wäßr. & wäßrig(e) \\
\hline
\end{tabular}




\section{Literaturverzeichnis}

[1] a) L. F. Tietze, U. Beifuss, Angew. Chem. 1993, 105, 137-170; Angew. Chem. Int. Ed. Engl. 1993, 32, 131-164; b) L. F. Tietze, Chem. Rev. 1996, 96, 115-136.

[2] B. M. Trost, Angew. Chem. 1995, 107, 285-307; Angew. Chem. Int. Ed. Engl. 1995, 32, 259-281.

[3] B. Kutscher, Bioforum 1996, 19, 17-23.

[4] a) R. B. Silverman, J. Seydel, „Medizinische Chemie“, VCH, Weinheim, 1994; b) „Medicinial Chemistry of the 21st Century“ (Hrsg.: C.G. Wermuth), Blackwell Scientific Publications, Oxford, 1992.

[5] F. Balkenhohl, C. von dem Bussche-Hünnefeld, A. Lansky, C. Zechel, Angew. Chem. 1996, 108, 2436-2502; Angew. Chem. Int. Ed. Engl. 1996, 35, 2288-2354.

[6] R. B. Merrifield, J. Am. Chem. Soc. 1963, 85, 2149-2153.

[7] Übersichtsartikel zu Peptid-Bibliotheken: a) C. Pinilla, S. Blondelle, C. Dooley, B. Dorner, J. Eichler, J. Ostresh, R. A. Houghten, Biopolymers (Pept. Sci.) 1995, 37, 221-248; b) G. Jung, A. G. Beck-Sickinger, Angew. Chem. 1992, 104, 375-412; Angew. Chem. Int. Ed. Engl. 1992, 31, 367-408; c) W. J. Dower, S. P. A Fodor, Annu. Rep. Med. Chem. 1991, 26, 271-299.

Übersichtsartikel zu Oligonucleotid-Bibliotheken: a) L. Gold, B. Polisky, O. Uhlenbeck, M. Yarus, Annu. Rev. Biochem. 1995, 64, 763-801; b) D. J. Ecker, T. A. Vikkers, R. Hanecak, V. Driver, K. Anderson, Nucleic Acids Res. 1993, 21, 1853-1869; c) L. C. Bock, L. C. Griffin, J. A. Latham, E. H. Vermaas, J. J. Toole, Nature 1992, 355, 564-570.

[8] J. M. Fréchet, Tetrahedron 1981, 37, 663-679.

[9] C. C. Leznoff, Acc. Chem. Res. 1978, 11, 327-335.

[10] M. A. Gallop, W. L. Fitch, Current Opinions in Chemical Biology 1997, 1, 94-121.

[11] a) W. L. Fitch, G. Detre, C. P. Holmes, J. N. Shoolery, P. A. Kiefer, J. Org. Chem. 1994, 59, 7955-7956; b) P. A. Kiefer, J. Org. Chem. 1996, 61, 1558-1560.

[12] C. L. Brummel, J. C. Vickerman, S. A. Carr, M. E. Hemling, G. D. Roberts, W. Johnson, J. Weinstock, D. Gaitanopoulos, S. J. Benkovic, N. Winograd, Anal. Chem. 1996, 68, 237-240.

[13] J. S. Früchtel, G. Jung, Angew. Chem. 1996, 108, 19-43; Angew. Chem. Int. Ed. Engl. 1995, 107, 17-40.

[14] a) A. Steinmetz, Dissertation 1997, Göttingen; b) L. F. Tietze, A. Steinmetz, Angew. Chem. 1996, 108, 682-683; Angew. Chem. Int. Ed. Engl. 1996, 35, 651-652. 
[15] a) „Combinatorial Solid Phase Organic Chemistry“, Advanced ChemTech Handbook, Louisville, USA, 1998; b) NovaBioChem, „Handbook and Catalog“, 1999.

[16] S. Mazur, P. Jayalekshmy, J. Am. Chem. Soc. 1979, 101, 677-680.

[17] N. K. Terrett, M. Gardner, D. W. Gordon, R. J. Kobylecki, J. Steele, Tetrahedron 1995, 51, 8135-8187.

[18] C. James, Tetrahedron 1999, 55, 4855-4946.

[19] ～L. A. Thompson, J. A. Ellman, Tetrahedron Lett. 1994, 35, 9333-9336.

[20] a) G. W. Kenner, J. R. McDermott, R. C. Sheppard, J. Chem. Soc. ,Chem. Commun. 1971, 636-637; b) B. J. Backes, J. A. Ellman, J. Am. Chem. Soc. 1994, 116, 11171 11172.

[21] M. J. Plunkett, J. A. Ellman, J. Org. Chem. 1995, 60, 6006-6007.

[22] a) I. Hughes, Tetrahedron Lett. 1996, 37, 7599-7603; b) B. A. Bunin, M. J. Plunkett, J. A. Ellman in „Combinatorial Peptide and Non-Peptide Libraries - A Handbook“ (Ed. G. Jung), VCH, Weinheim, 1996, 405-415.

[23] C. P. Holmes, D. G. Jones, J. Org. Chem. 1995, 60, 2318-2319.

[24] a) L. A. Thompson, J. A. Ellman, Chem. Rev. 1996, 96, 555-602; b) J. Alper, Science 1994, 264, 1399-1412; c) M. A. Gallop, R. W. Barret, W. J. Dower, S. P. A. Fordor, E. M. Gordon, J. Med. Chem. 1994, 37, 1232-1280; d) M. R. Pavia, T. K. Sawyer, W. H. Moos, Bioorg. Med. Chem. Lett. 1993, 3, 387-403, e) R. C. D. Brown, J. Chem. Soc, Perkin, Trans. 1 1998, 3293-3320.

[25] a) S. Cheng, D. D. Comer, J. P. Williams, P. L. Myers, D. L. Boger, J. Am. Chem. Soc. 1996, 118, 2567-2569; b) M. C. Pirrung, J. H.-L. Chau, J. Chen, Chem. Biol. 1995, 2, 621-623; c) T. Carell, E. A. Wintner, A. Bashir-Hashemi, J. Rebek, Jr., Angew. Chem. 1994, 107, 2159-2160; Angew. Chem. Int. Ed. Engl. 1994, 33, $2159-$ 2160; d) T. A. Keating, R. W. Armstrong, J. Am. Chem. Soc. 1995, 117, 7842-7846.

[26] A. Furka, F. Sebestyen, M. Asgedom, G. Dibo, Int. J. Pept. Protein Res. 1991, 37, 487-492.

[27] a) A. R. Brown, P. H. H. Hermkens, H. C. J. Ottenheijm, D. C. Rees, Synlett 1998, 817-827; b) P. H. H. Hermkens, H. C. J. Ottenheijm, D. C. Rees, Tetrahedron 1997, $53,5643-5678$.

[28] Persönliche Mitteilung, BMBF-Projektgruppentreffen „Kombinatorische Chemie“, Juni 1999.

[29] M. J. Plunkett, J. A. Ellman, J. Am. Chem. Soc. 1995, 117, 3306-3308.

[30] G. C. Look, J. R. Schullek, C. P. Holmes, J. P Chinn, E. M. Gordon, M. A. Gallop, Bioorg. Med. Chem. Lett. 1996, 6, 707-712.

[31] E. Mutschler, „Arzneimittelwirkungen“, WVG, Stuttgart, 1997. 
[32] C. Watson, Angew. Chem. 1999, 111, 2025-2031; Angew. Chem. Int. Ed. Engl. 1999, 38, 1903-1908.

[33] K. C. Nicolaou, D. Vourloumis, T. Li, J. Pastor, N. Winssinger, Y. He, S. Ninkovic, F. Sarabia, H. Vallberg, F. Roschangar, N. P. King, M. R. V, Finlay, P. Giannakakou, P. Verdier-Pinard, E. Hamel, Angew. Chem. 1997, 109, 2181-2187; Angew. Chem. Int. Ed. Engl. 1997, 36, 2097-2103.

[34] K. C. Nicolaou, N. Winssinger, J. Pastor, F. Murphy, Angew. Chem. 1998, 110, 2677-22680; Angew. Chem. Int. Ed. Engl. 1998, 37, 2534-2537.

[35] a) L. F. Tietze, A. Steinmetz, Synlett 1996, 667-668; b) L. F. Tietze, A. Steinmetz, F. Balkenhohl, Bioorg. Med. Chem. Lett. 1997, 7, 1303-1310.

[36] a) J. E. Bäckvall, B. Akermark, S. O. Ljunggeren, J. Am. Chem. Soc. 1979, 101, 2411-2423; b) J. E. Bäckvall, Acc. Chem. Res. 1983, 16, 335-342; c) I. Tsuji, Synthesis 1984, 369-381.

[37] a) R. Mozingo, Org. Syn., Coll. Vol 3 1955, 685-735; b) L. F. Fieser, M. Fieser, „Reagents for Organic Synthesis“, Wiley \& Sons, New York, 1962, 778-821; c) N. A. Cortese, R. F. Heck, J. Org. Chem 1978, 43, 3985-4005.

[38] J.-L. Malleron, J.-C. Fiaud, J.-Y. Legros, ,Handbook of Palladium-Catalyzed Organic Reactions “, Academic Press, San Diego, 1997.

[39] J. Tsuji, H. Takahashi, M. Morikawa, Tetrahedron Lett. 1965, 6, 4387-4388.

[40] a) B. M. Trost, D. L. Van Vranken, Chem. Rev. 1996, 96, 395. b) C. G. Frost, J. Howath, J. Williams, Tetrahedron: Asymmetry 1992, 3, 1089. c) J. Tsuji, Tetrahedron 1986, 42, 4361; d) S. A. Godleski in Comprehensive Organic Synthesis, Vol. 4 (Hrsg. B. M. Trost), Pergamon Press, Oxford, 1991, 585-661.

[41] a) T. Hayashi in „Catalytic Asymmetric Synthesis“ (Hrsg. I. Ojima), Verlag Chemie, Weinheim, 1993, 325-365; b) B. M. Trost, Acc. Chem. Res. 1996, 29, 355-364.

[42] a) T. Yamamoto, M. Akimoto, O. Saito, A. Yamamoto, Organometallics 1986, 5, 1559-1561; b) H. Kurosawa, J. Organomet. Chem. 1987, 334, 243-253.

[43] J. C. Fiaud, J. Y. Legros, J. Org. Chem. 1987, 52, 1907-1911.

[44] „Hard-Soft-Acid-Base“-(HSAB)-Prinzip: T. L. Ho, „Hard and soft acids and bases principle in organic chemistry“, Academic Press, New York, 1977.

[45] Die syn-anti-Nomenklatur bezieht sich auf die endständigen Substituenten und das Wasserstoffatom in 2-Position.

[46] J. Tsuji, I. Minami, Acc. Chem. Res. 1987, 20, 140-145.

[47] R. F. Heck, J. Am. Chem. Soc. 1968, 90, 5518-5520.

[48] Übersichten: a) R. F. Heck, Palladium Reagents in Organic Synthesis, Academic Press, New York 1985; b) A. de Meijere, F. E. Meyer, Angew. Chem. 1994, 106, 2473-2506; Angew. Chem. Int. Ed. Engl. 1994, 33, 2379-2412; c) J. Tsuji, Palladium 
Reagents and Catalysts: Innovations in Organic Synthesis, Wiley, New York 1995; d) E.-I. Negishi, C. Copéret, S. Ma, S.-Y. Liou, F. Liu, Chem. Rev. 1996, 96, 365393; e) S. Bräse, A. de Meijere in „Metal-catalyzed Cross-coupling Reactions“ (Hrsg. F. Diederich, P. J. Stang), Wiley-VCH, Weinheim 1998, S. 99-166; f) W. Capri, I. Candiani, Acc. Chem. Res. 1995, 28, 2-12.

[49] R. McCrindle, G. Ferguson, G. J. Arsenault, A. J. McAlees, J. Chem. Soc., Chem. Commun. 1983, 571-572.

[50] a) W. A. Herrmann, C. Broßmer, K. Öfele, C.-P. Reisinger, T. Priermeier, M. Beller, H. Fischer, Angew. Chem. 1995, 107, 1989-1992; Angew. Chem Int. Ed. Engl. 1995, 34, 1844-1846; b) M. Beller, H. Fischer, W. A. Herrmann, K. Öfele, C. Broßmer, Angew. Chem. 1995, 107, 1992-1993; Angew. Chem Int. Ed. Engl. 1995, 34, 18461847; c) W. A. Herrmann, C. Broßmer, C.-P. Reisinger, T. H. Riermeier, K. Öfele, M. Beller, Chem. Eur. J. 1997, 3, 1357-1359; d) M. Beller, T. H. Riermeier, C.-P. Reisinger, W. A. Herrmann, Tetrahedron Lett. 1997, 38, 2073-2074.

[51] G. P. C. M. Decker, C. J. Elsevier, K. Vrieze, P. W. N. M. van Leeuwen, Organometallics 1992, 11, 1598-1603.

[52] C. Y. Hong, N. Kado, L. E. Overman, J. Am. Chem. Soc. 1993, 115, 11028-11029.

[53] A. Ashimori, T. Matsuura, L. E. Overman, D. J. Poon, J. Org. Chem. 1993, 58, 6949-6951.

[54] a) T. Nöbel, Dissertation 1998, Göttingen; b) L. F. Tietze, T. Nöbel, M. Spescha, Angew. Chem. 1996, 108, 2385-2386; Angew. Chem. Int Ed. Engl. 1996, 35, 23702371.

[55] B. M. Trost, Angew. Chem. 1989, 101, 1199-1201; Angew. Chem. Int Ed. Engl. 1989, 28, 1188-1190.

[56] B. M. Trost, R. Madsen, S. D. Guile, Tetrahedron Lett. 1997, 38, 1707-1710.

[57] a) H. Schirok, Dissertation 1999, Göttingen; b) L. F. Tietze, H. Schirok, Angew. Chem. 1997, 109, 1159-1160; Angew. Chem. Int. Ed. Engl. 1997, 36, 1148-1149.

[58] H. Laatsch, „Die Technik der organischen Trennungsanalyse“, Georg Thieme Verlag, Stuttgart, 1988.

[59] a) T. Hippe, Diplomarbeit 1996, Göttingen; b) L. F. Tietze, T. Hippe, A. Steinmetz, Synlett 1996, 1043-1044.

[60] J. S. Witzeman, W. D. Nottingham, J. Org. Chem. 1991, 56, 1713-1716.

[61] B. M. Trost, D. J. Murphy, Organometalics 1985, 4, 1143-1145.

[62] Die Synthese von 218 erfolgte durch Erhitzen von spacermodifiziertem MerrifieldHarz 6 mit 4-Phenylacetessigsäure-ethylester für 24 Stunden am Rückfluß in Toluol. Für die Überlassung der Verbindung 218 danke ich Frau Dipl.-Chem. Nadège Labuche. 
[63] „Organikum“, Verlag der Wissenschaften, Berlin, 1990, 477.

[64] „Organikum“, Verlag der Wissenschaften, Berlin, 1990, 416.

[65] „The Chemistry of Heterocyclic Compounds“, Vol. 20, R. H. Wiley and P. Wiley, „Pyrazolones, Pyrazolidones and Derivatives“, Interscience Publishers, New York, 1964.

[66] Für die freundliche Überlassung der Hydrazine danke ich Herrn Dr. Markus Menges, BASF-AG.

[67] a) T. Eicher, S. Hauptmann, „Chemie der Heterocyclen“, Georg Thieme Verlag, Stuttgart, 1994, 187 und dort zitierte Literatur; b) Eluguero, Marzin, Katritzky, Linda, „The Tautomerism of Heterocycles“, Academic Press, New York, 1976, 314.

[68] Es sind nur die der polymergebundenen Verbindung eindeutig zugewiesenen Signale angegeben. Ansonsten wird das bandenreiche Spektrum des Polystyrol-Grundkörpers beobachtet (siehe Kapitel 2.1.).

[69] Für die freundliche Überlassung des entsprechenden Allylalkohols danke ich Herrn Dipl.-Chem. Jürgen Seibel.

[70] Für die freundliche Überlassung des entsprechenden Allylalkohols danke ich Frau Katja Heitmann. 


\section{Danksagungen}

Für die Aufnahme der NMR-Spektren danke ich Herrn R. Machinek stellvertretend für die NMR-Abteilung des Instituts. Herrn Dr. G. Remberg und Frau G. Udvarnoki möchte ich für die Aufnahme der Massenspektren und Herrn M. Beller für die Durchführung der Elementaranalysen danken. Mein besonderer Dank gilt Frau E. Pfeil für die Aufnahme der zahlreichen IRund UV-Spektren.

Der gesamten Abteilung von Prof. L. F. Tietze danke ich für die angenehme Arbeitsatmosphäre und die stete Hilfsbereitschaft. Dies gilt insbesondere für die alte Besatzung von „434“, den Herrn Dipl.-Chem. Wolf Hees sowie den Herren Dr. Christian Janßen, Thomas Nöbel und Christian Wulff. Für die nette Aufnahme als „Dienstältesten“ in meinem neuen Labor „402“ danke ich den Damen Katja Heitmann und Katja Schäfer sowie den Herren Dipl.-Chem. Thomas Buck, Ulrich Grießbach, Rudi Krahnert, Lars Lücke, Oliver Meyer, Klaus Schrader und Christoph Tappertshofen.

Für das engagierte Korrekturlesen meiner Arbeit danke ich den Herren Dipl.-Chem. Ulrich Grießbach, Chistoph Tappertzhofen, Enno Töpke, Ludwig Völkel und Michael Wöhrmann.

Danken möchte ich auch dem Bundesministerium für Bildung, Wissenschaft, Forschung und Technologie (BMBF-Projekt „Kombinatorische Chemie“, Förderkennzeichen 03 D0056 2) und der BASF AG, Ludwigshafen, für finanzielle Unterstützung.

Last, but not least gilt mein ganz besonderer Dank Nadège für ... einfach alles.

Meine akademischen Lehrer waren u. a. die Herren Professoren und Dozenten J. Belzner, H. Gründig, W. Hack, G. F. Kahl, H. Laatsch, K. Luther, A. de Meijere, A. Meller, G. von Minnigerode, E. Puschmann, H. W. Roesky, J. Schröder, E. Schwarzmann, L. F. Tietze, D. Treciok, H. G. Wagner. 


\section{Lebenslauf}

\section{Persönliche Daten}

Name:

Thomas Hippe

Geburtsdatum/-ort:

29.10.1970 in Göttingen

Eltern:

Dr. Hans Hippe, Mikrobiologe

Edeltraut Hippe, geb. Peters, Lehrerin

\section{Schulausbildung}

$1977-1981$

$1981-1983$

$1983-1990$

28.05 .1990

\section{Wehrdienst}

$1990-1991$

\section{Studium}

Oktober 1991

08.07.1994

Januar 1996 -

September 1996

01.11 .1996

Januar 1997 -

September 1999

02.11 .1999

\author{
Adolf-Reichwein-Schule, Göttingen \\ Bert-Brecht-Schule, Göttingen \\ Felix-Klein-Gymnasium, Göttingen \\ Schulabschluß mit der Allgemeinen Hochschulreife
}

Beginn des Studiums der Chemie an der Georg-AugustUniversität Göttingen

Diplom-Chemiker-Vorprüfung

Diplomarbeit am Institut für Organische Chemie der GeorgAugust-Universität Göttingen im Arbeitskreis von Prof. Dr. Dr.

h. c. L. F. Tietze über „Festphasensynthese von 3,4-Dihydro2H-pyranen und ihre Anwendung in der Kombinatorischen Chemie"

Diplom-Chemiker-Hauptprüfung

Dissertation am Institut für Organische Chemie der Georg-August-Universität Göttingen im Arbeitskreis von Prof. Dr. Dr. h. c. L. F. Tietze über „Palladiumkatalysierte allylische Alkylierung und Synthese von Pyrazolonen auf der festen Phase“"

Tag der mündlichen Prüfung 